\title{
Chemical Sensing with 2D Materials
}

Cosimo Anichini, ${ }^{1, \#}$ Włodzimierz Czepa, ${ }^{2,3, \#}$ Dawid Pakulski, ${ }^{1,2,3, \#}$ Alessandro Aliprandi, ${ }^{1}$ Artur Ciesielski, ${ }^{1, *}$ Paolo Samorì ${ }^{1, *}$

${ }^{1}$ Université de Strasbourg, CNRS, ISIS, 8 alleé Gaspard Monge, 67000 Strasbourg, France. Email : samori@unistra.fr, ciesielski@unistra.fr

${ }^{2}$ Faculty of Chemistry, Adam Mickiewicz University, Umultowska 89b, 61614 Poznań, Poland

${ }^{3}$ Centre for Advanced Technologies, Adam Mickiewicz University, Umultowska 89c, 61614 Poznań, Poland

\# These authors contributed equally to this work 


\begin{abstract}
During the last decade, two-dimensional materials (2DMs) have attracted a great attention due to their unique chemical and physical properties, which make them appealing platforms for diverse applications in opto-electronic devices, energy generation and storage, and sensing. Among their various extraordinary properties, 2DMs possess high surface-to-volume ratio and ultra-high surface sensitivity to the environment, being key characteristics for applications in chemical sensing. Furthermore, 2DMs' superior electrical and optical properties, combined with excellent mechanical characteristics such as robustness and flexibility, render these materials ideal components for the fabrication of a new generation of high-performance chemical sensors. Depending on the specific device, 2DMs can be tailored to interact with various chemical species at the non-covalent level making them powerful platforms for fabricating devices expressing a high sensitivity towards detection of various analytes including gas, ions and small biomolecules. Here, we will review the most enlightening recent advances in the field of chemical sensors based on atomically-thin 2DMs and we will discuss the opportunities and the challenges towards the realization of novel hybrid materials and sensing devices.
\end{abstract}




\section{Introduction}

Since the ground-breaking experiments by Geim and Novoselov on the isolation and study of the outstanding physical properties of graphene in $2004,{ }^{1}$ the research endeavour on twodimensional materials (2DMs) have grown exponentially becoming a flagship in many classical fields of research such as chemistry and condensed matter physics, and it is particularly blooming in the interdisciplinary realms of nanoscience and material science. One-atom thick graphene is arguably the most glorified material of the last decade; its fascinating physico-chemical properties have spread beyond the academic community and drew attention of world-leading chemical and materials oriented companies as well as public institutions, especially since graphene-based products are going on sale. ${ }^{2}$ This so-called 'graphene rush' has triggered the pursuit of atomically thin sheets of other layered materials, such as semiconducting transition metal dichalcogenides (TMDs), ${ }^{3}$ boron nitride and, more recently, MXenes which include transition metal carbides, nitrides and carbonitrides, ${ }^{4}$ and phosphorene..$^{5-7}$ The chemical and structural diversity of these 2DMs, whose properties are indeed dictated by their dimensionality, ${ }^{8}$ offer immense opportunities for fundamental and applied research. Different optical and electronic properties may be obtained ranging from the exceptional semi-metallic conductivity of graphene, 10 to the semiconducting characteristics of some TMDs that possess sizeable and tuneable bandgaps, which change from indirect (bulk material) to direct (single layer form). ${ }^{11}$ This results also in unique photoluminescent properties, ${ }^{12}$ thus making such materials suitable for diverse applications such as transistors, photodetectors, electroluminescent devices and luminescence probes. The richness of electronic and optical properties, which can be also engineered by chemical functionalization, combined with their 2D nature, i.e. their extremely high surface-to-volume ratio, make such materials extremely appealing in the field of sensing, ranging from the quantitative detection of gas, metal (alkali and heavy metal), to biologically relevant molecules (e.g. glucose and DNA). Noteworthy, unlike classical digital sensors, 2DMs-based sensors do not possess physical gates for selectively reacting to the targeted species (gas molecules, ${ }^{13,14}$ metal ions or biomolecules ${ }^{15,16}$ ).

The interaction between 2DMs' sheets and molecules/ions is accompanied by the adjustment of the properties of both initial components. Such interaction can occur via the physical adsorption, i.e. physisorption, of molecular units onto basal plane of 2DMs' sheets through non-covalent interactions, or through the chemical adsorption, i.e. chemisorption, of reactive 
species undergoing chemical reactions with 2DMs to form covalent bonds onto their basal planes. In the field of sensing non-covalent interactions may be preferred when a quick response and a fast recovery rate is required (i.e. real-time monitoring), yet the weakness of the supramolecular forces can be disadvantageous when biomolecules (i.e. enzyme) need to be immobilized on the surface and to be stable during the assay (i.e. in buffered saline solution), thus making covalent linkage more suitable.

The physisorption of molecules onto surface depends on the nature of both analyte and surface; for example, graphene is an extended honeycomb network of $\mathrm{sp}^{2}$ hybridized carbon atoms characterized by a long-range $\pi$-conjugation. Consequently, non-covalent intermolecular interactions involving $\pi$-systems are pivotal in the recognition events since subtle changes in the electronic characteristics of the $\pi$ systems can lead to modifications of the structure and properties, ${ }^{17,18}$ as well as they may enhance the stability of the physisorbed compounds as observed for proteins, enzyme-drug and DNA-protein complexes. ${ }^{19,}{ }^{20}$ The understanding of the nature of $\pi$-complexes has indeed high importance for graphene based sensors since the gas $-\pi$ interaction, ${ }^{21} \mathrm{H}-\pi$ interaction, ${ }^{22-25} \pi-\pi$ interaction, ${ }^{26-31}$ cation- $\pi$ interaction, ${ }^{32-39}$ and anion $-\pi$ interaction ${ }^{26,40-50}$ possess different strength which is determined by a combination of attractive and repulsive forces. Compared to graphene, the family of TMDs, which includes molybdenum disulfide $\left(\mathrm{MoS}_{2}\right)$, tungsten disulfide $\left(\mathrm{WS}_{2}\right)$, molybdenum diselenide $\left(\mathrm{MoSe}_{2}\right)$ and tungsten diselenide $\left(\mathrm{WSe}_{2}\right)$ as the most studied, has not been investigated extensively from this point of view. However, for the case of $\mathrm{MoS}_{2}$ it has been demonstrated that the physisorption onto its basal plane is mainly driven electromagnetic interactions (e.g. electrostatic and van der Waals). ${ }^{51,52}$ More generally, 2DMs produced by means of different methods can be very different due to the presence of structural defects which leads to different behaviours, and consequently different performance in the final sensing device. In the last years the term "defects" keyword in the field of 2DMs since the presence of defects play a major role in modifying the properties of 2DMs. Although it might have a negative implication when one targets applications in fast opto-electronics, well-designed defects might lead to new and tuneable properties opening a wide range of interesting applications that pristine materials cannot afford such as enhanced electron transfer rate and electrochemical activity as observed for graphene based electrochemical sensors. ${ }^{58}$ Lattice vacancies affect the electronic properties of the TMDs sheets, by lowering their charge carrier mobility and density ${ }^{59,60}$ as well as triggering photoluminescence ${ }^{61,62}$ and modifying chemical reactivity. ${ }^{63}$ In graphene the 
defects within the honeycomb network of $\mathrm{C}=\mathrm{C}$, whose number depends mainly on the protocol employed for its fabrication, typically consists in point defects, i.e. the absence some $\mathrm{sp}^{2}$ carbon atoms, and/or the presence of carbon atoms with $\mathrm{sp}^{3}$ hybridization. The carbon atoms surrounding these defects are electronically perturbed thus they exhibit different electronic structures; therefore, they are chemically activated for further chemical reactions. In other words, the presence of points defects enhances the chemical reactivity of graphene. ${ }^{64}$ $\mathrm{sp}^{2}$ carbon atoms of graphene can react with highly reactive free radicals, such as those produced by diazonium salts ${ }^{65-67}$ or benzoyl peroxide; ${ }^{68}$ as well as dienophiles which react with $\mathrm{C}=\mathrm{C}$ of graphene through a 1,3 dipolar cycloaddition. ${ }^{69}$ However, a massive introduction of defects can be achieved by exposing graphite to strong oxidizing agents ${ }^{70}$ resulting in the formation of graphite oxide (GO). The latter when immersed in water tends to spontaneously exfoliate into single layers of graphene oxide due to the negative charged oxygen functionalities decorating both basal plane and edges. ${ }^{71}$ Such material is probably the most investigated $2 \mathrm{DM}$ in the field of sensing ${ }^{72-76}$ because of its ease functionalization. The negative charges on its surface can interact non-covalently with a wide range of positively charged molecules; for example, outstanding sorption properties for heavy metal cations have been reported. ${ }^{77,}{ }^{78}$ Moreover, the oxygen functionalities, which consist mostly of hydroxyl and epoxy groups exposed on the basal plane and carboxy and carbonyl functionalities located at the sheet edges, ${ }^{79,80}$ are extremely reactive thus allowing further modification of GO. ${ }^{81,82}$ Importantly, the electrical conductivity can be to a great extent restored and tuned during the reduction step of the functionalized graphene oxide (fGO) ${ }^{83}$ making it an extremely versatile material for sensing with electrical read out. The use of defects to introduce functionalities has been also successfully extended to the family of TMDs where the presence of chalcogen deficiency can be used to decorate the surface of the 2DM with different functionalities. For example reactive sulphur vacancies in $\mathrm{MoS}_{2}$ may simply arise during the exfoliation process ${ }^{59,84}$ or be introduced on purpose electrochemically ${ }^{85}$ or by ionbeam irradiation. ${ }^{86}$ These reactive sites can readily react with sulphur-containing moieties such as alkanethiol molecules resulting into a covalent bond formation. ${ }^{86-88}$ Alternatively, defects can be further expanded into (sub)-nanometre sized pores transforming the 2DMs into permselective membranes ${ }^{89}$ or ultrasensitive sensors even able to sequence DNA. ${ }^{90,}{ }^{91}$ Such property can be further tuned and harnessed to achieve enhanced permselectivity by controlled chemical functionalization of both pore edges and surface in a post-process chemical treatment. ${ }^{89}$ As already anticipated the $2 \mathrm{D}$ nature of such materials offers several advantages in the field of sensing since the atomic thickness provides a direct interaction of 
all the atoms with the analyte while the large lateral size not only guarantees a large active surface for the sensing event, but it also facilitates the assembly of the device, as demonstrated for field-effect transistor (FETs), i.e. by enabling a better contact with metal electrodes and better control over the channel structures. ${ }^{92}$ Obviously, the architecture of the sensing device depends on which properties of the $2 \mathrm{DMs}$ the analytes is affecting mostly as well as on the nature of both the 2DM and the analyte. Electrochemical sensors based on graphene have been probably the most investigated so far since they provide a direct electrical response. Graphene ${ }^{93}$ offers indeed a large electrochemical window (up to $2.5 \mathrm{~V}$ ), ${ }^{72}$ thus enabling the detection of molecules with high reduction or oxidation potential (e.g. nucleic acids), and good electrocatalytic activities for many redox reactions. ${ }^{94}$ Furthermore, due its ambipolar character, the functionalization with both electron withdrawing or donating groups can lead to chemical gating resulting in a change of conductivity of the material. ${ }^{95}$ The combination of the atomic thickness of the 2DMs with the chemical gating that results when the surface potential is changed due to the binding of molecules has led to the generation of new FETs sensors based on 2DMs, as recently reviewed by Mao et al. ${ }^{92}$ Interestingly in such a kind of sensors, 2D semiconducting materials outperform their conducting counterparts since the presence of a finite band gap decreases the initial conductance inside the channel thus improving the signal-to-noise ratio. Consequently, the performance of the device is mainly dictated by the band gap which can be tuned by defects engineering, doping, as well as by playing with the thickness of the material opening a wide range of opportunities as recently demonstrated by Cui et al. ${ }^{96}$ in a phosphorene-based FET gas sensor.

While a direct electrical response is generally preferred for practical applications, the interactions of the 2DMs with analytes give rise to interesting optical phenomena such as the modulation of the photoluminescent properties opening up a wide range of opportunities. Graphene and GO are known to be highly efficient fluorescence quenchers if compared to organic compounds, thus Förster resonance energy transfer (FRET) sensors have attracted increasing interest in the last years especially for biomedical applications since they can be used to measure precisely nanometre-scale distance and changes both in vivo and in vitro ${ }^{97}$ resulting into nanobiosensors with excellent sensitivity, selectivity, and biostability. ${ }^{98}$ Even single-layer $\mathrm{MoS}_{2}$ nanosheet possesses high fluorescence quenching efficiency and by taking advantage of such a characteristic it has been exploited as sensing platforms for the detection of DNA and small molecules. ${ }^{99}$ However, the use of 2DMs in FRET is not limited to energy acceptor since a proper functionalization may result in photoluminescent flakes which can act 
as energy donor and be quenched by more electron deficient molecules such as nitro compounds which are common constituent to prepare powerful explosives. ${ }^{100}$

The use of 2DMs as substrate for enhancing the Raman signals of adsorbed molecules represented a major breakthrough in the field of sensing. ${ }^{101,102}$ Different Raman vibrational modes can be enhanced depending on which layered material the molecule is adsorbed onto. Surface Enhanced Raman Spectroscopy (SERS) is mainly employed to explore the detection of chemical and biological species ${ }^{103-106}$ due its high sensitivity (even down to single molecules) $)^{107,108}$ and the bar-code like reading that comes from the narrow vibrational bands in the Raman spectrum. Different Raman enhancement mechanisms have been proposed for different 2DMs, however, like in the previous examples, surface modification ${ }^{109}$ as well as the thickness ${ }^{110}$ play a fundamental role in terms of selectivity and Raman signal enhancement.

The intensive research on 2DMs for sensing application has been further motivated by their intrinsic mechanical properties such as robustness, flexibility, lightweight which makes possible the realization of portable and wearable sensors with tremendous impact on our society enabling the monitoring of the wearers' health, fitness, and their surroundings. ${ }^{111}$ The development of wearable chemical sensors faces multiple challenges on various fronts such as power, analytical procedure, communication, data acquisition, processing and security. Nevertheless, several examples of flexible graphene-based wearable gas and chemical sensors has been recently reviewed ${ }^{112}$ and also a wearable patch for sweat-based diabetes monitoring and feedback therapy has been reported, ${ }^{113}$ which combines a heater, temperature, humidity, glucose and $\mathrm{pH}$ detection.

Looking forward to the emergence of portable and ultrasensitive sensors based on 2DMs, in this Review article we discuss the recent advances in gas, alkali and heavy metals sensing as well as relevant chemical entities, emphasizing the performance of the different sensor devices based on 2DMs in terms of sensitivity, selectivity, robustness and response time, by focusing on the device preparation and their suitability as wearable sensors. Each section will start with a general introduction to the sensing towards a specific analyte (gas, metals or chemically relevant molecules), and will follow with a detailed discussion on the smartest approached strategies and the best recent achievements obtained for various 2DMs ranging from graphene to MXenes. For each 2DM we classify the sensors on the basis of the type of signal transduction; in particular, we focus on electrochemical, FET, fluorescence and SERS 
sensors. While presenting remarkable examples of chemical sensors we will provide specific attention to the most important figures of merit such as the sensitivity, selectivity, robustness, response time, and to the use of strategies to minimize and ideally exclude effects of interfering analytes, with the final aim of developing flexible sensors for wearable technology.
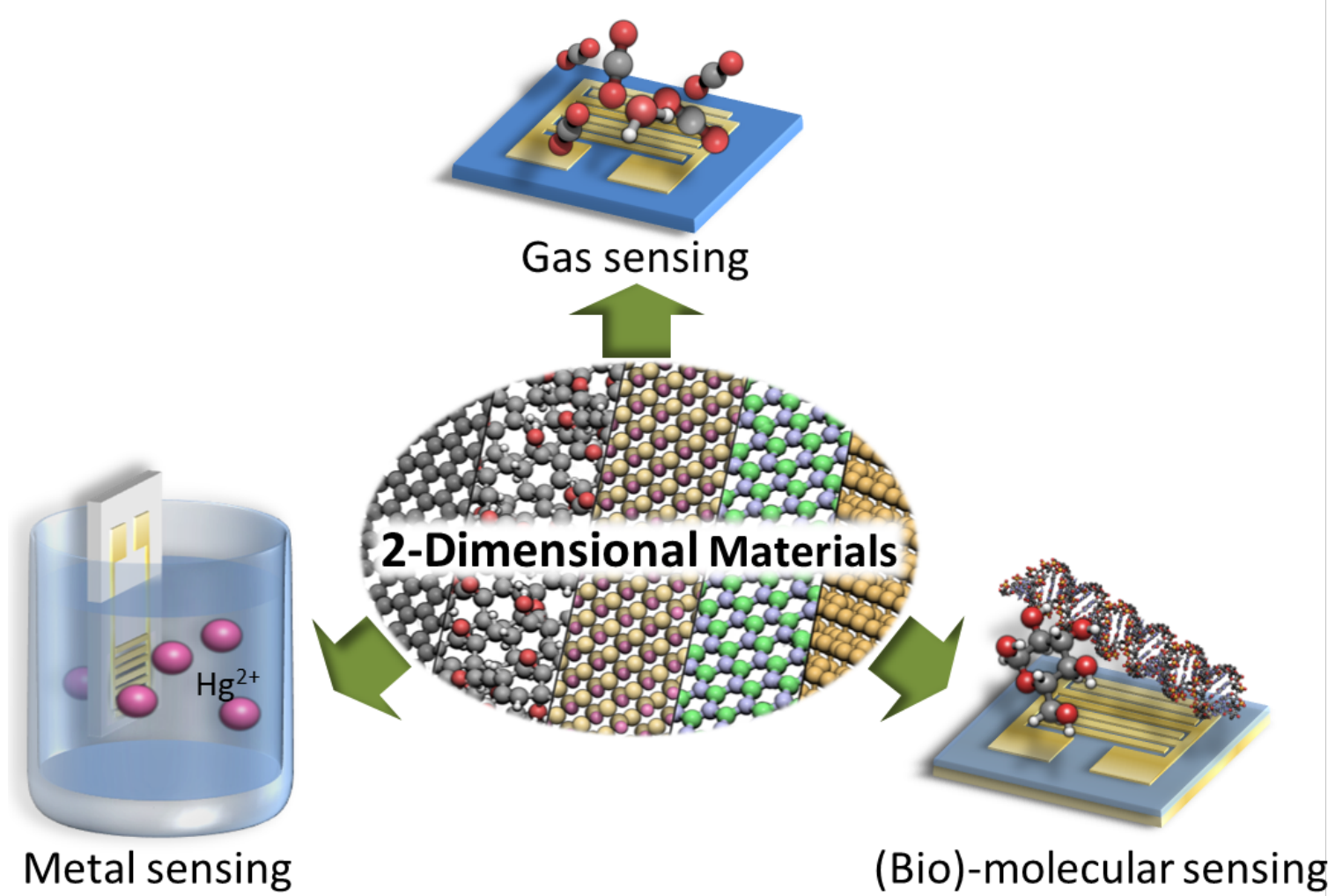

Figure 1: Schematic representation of the different molecular chemical sensors based on 2DMs approaches that have been explored over the last years.

\subsection{Overview on the properties of 2DMs}

Graphene is an atomically thin, planar membrane of carbon atoms arranged in a honeycomb lattice whose unique properties were firstly investigated by Geim and Novoselov in 2004. ${ }^{1}$ Graphene can be seen as a single layer of graphite, and while the latter behaves metallically, graphene is a semi-metal featuring a unique zero band gap. ${ }^{114}$ In addition graphene exhibits remarkable thermal and electrical conductivity and an impressive mechanical strength being 
superior to steel. ${ }^{2}$ Graphene is particularly promising for sensing by virtue of its extremely high conductivity, and its large surface area. Despite being only one atom thick graphene is impermeable in its pristine form, effectively blocking the passage of even the smallest molecules. ${ }^{115}$ The impermeable nature of graphene has triggered extensive studies on its application as a barrier for liquid and gas permeation, ${ }^{116,117}$ as well as on its use as shielding material protecting metallic surfaces against corrosion. ${ }^{118}$ Such unique property of graphene has triggered extensive efforts towards the use graphene and other 2DMs for the design of ultrathin water-separation membranes and as platforms to absorb (heavy) metal ions. Hitherto the highest quality graphene flakes have been obtained by using a top-down approach relying on the mechanical exfoliation. The graphene layers are most commonly exfoliated from the bulk graphite via the "scotch tape" method. ${ }^{119}$ Unfortunately, the flakes produced with such a methods have high quality, yet very limited lateral size. Large area and high-quality monoand few-layer thick graphene sheets can be obtained by two bottom-up approaches: the chemical vapour deposition (CVD) and the epitaxial growth. CVD graphene is usually obtained through a catalytical decomposition of hydrocarbons (usually methane) on a hot $\left(\sim 1000{ }^{\circ} \mathrm{C}\right)$ metal surface $(\mathrm{Cu}, \mathrm{Ni}$ and $\mathrm{Co})$ under vacuum. ${ }^{120}$ Since the graphene grows directly onto the metal surface, different techniques have been developed to transfer it onto dielectric substrates. ${ }^{121}$ Epitaxial growth is another method to obtain large and uniform highquality graphene films. ${ }^{122}$ Typically, $\mathrm{SiC}$ is heated under high vacuum at high temperature ( $>$ $1200{ }^{\circ} \mathrm{C}$ ); this allows the surface silicon atoms to evaporate, yielding the rearrangement of the carbon atoms to form a graphene layer. Mechanical exfoliation makes it possible to obtain high quality graphene monolayers, but the very low throughput and yield of the soobtained graphene flakes hinder any industrial application, while CVD and epitaxial grow allow to obtain large-area graphene monolayers, although the production costs remain high. The production of GO followed by its reduction is a high throughput, easily scalable and cheap method to obtain large amount of graphene. ${ }^{123}$ It is however fair to note that reduced graphene oxide (rGO) is less conductive and have numerous structural defects and residual functional groups compared to pristine graphene, yet their presence could offer a clear route for improvement of the sensing capabilities as already discussed. ${ }^{124}$ Similarly GO has a higher number of oxygen functional groups, but it is an electrical insulator. ${ }^{123}$ A compromise in terms of quality of the flakes and yield is offered by the liquid-phase exfoliation (LPE) of graphite: graphene flakes dispersed in water/surfactants or organic solvents can be obtained with the aid of ultrasonication, ${ }^{125,126}$ shear mixing ${ }^{127}$ or electrochemical approach. ${ }^{128}$ 
Alongside graphene, transition metal dichalcogenides are the most studied 2DMs. TMDs are semiconductors of the type $\mathrm{MX}_{2}$, where $\mathrm{M}$ is a transition metal atom (such as Mo, W, Re and others belonging to the 4,5 and 10 group) and $\mathrm{X}$ is a chalcogen atom (such as $\mathrm{S}, \mathrm{Se}$ or Te); these materials that exist in the bulk form can be exfoliated yielding 2D monolayers in which one atomic layer of the metal is sandwiched between two layers of $\mathrm{X}$ atoms. TMDs are promising materials for the use in electronic devices, ${ }^{129,130}$ energy storage ${ }^{131}$ and sensors ${ }^{132-}$ ${ }^{134}$ due to their unique chemical and physical properties, including semiconducting properties, high surface-to-volume ratios and absorption coefficients, adjustable and direct band gaps and availability of reactive sites for redox reactions. ${ }^{135}$ The chemical versatility of TMDs and their reactivity together with their natural abundance result in the ever growing interest in those materials. ${ }^{135}$ Although majority of TMDs exhibit similar structure, the opto-electronic properties are diverse. In particular, the band gap of TMDs ranges from the insulating $\mathrm{HfS}_{2}$ to the conductive $\mathrm{VSe}_{2}{ }^{135}$ Noteworthy, 2D TMDs exhibits two different crystal phases: a trigonal prismatic and a octahedral phase, usually denoted as $2 \mathrm{H}$ and $1 \mathrm{~T}$ phases, respectively. In Mo- and W-based TMDs the $2 \mathrm{H}$ phase is thermodynamically stable and has semiconductive properties, while the 1T phase displays metallic properties. Other TMDs present a stable distorted octahedral phase, usually referred as $1 \mathrm{~T}^{\prime}$ phase. ${ }^{136}$ The most investigated TDMs are $\mathrm{MoS}_{2}, \mathrm{MoSe}_{2}, \mathrm{WS}_{2}, \mathrm{WSe}_{2}, \mathrm{ReS}_{2}$ and $\mathrm{ReSe}_{2}$. Among these, $\mathrm{MoS}_{2}$ is considered as the most promising material as it possesses various physico-chemical properties including fast electron transfer, good conductivity and quenching ability and it is abundant in nature as molybdenite. Multiple preparation techniques were reported to obtain nanosized $\mathrm{MoS}_{2}$ including $\mathrm{LPE},{ }^{137}$ chemical vapour deposition, ${ }^{138,}{ }^{139}$ lithium intercalation. ${ }^{99}$, 140, 141 LPE makes it possible to obtain dispersion of $\mathrm{MoS}_{2}$ nanosheets and other TMDs in higher yield and low costs. However, the obtained materials are usually quite thick and feature limited lateral dimensions. ${ }^{142}$ Larger mono and few layers thick $\mathrm{MoS}_{2}$ of good quality can be produced by $\mathrm{CVD}^{143}$ directly on various substrates, including $\mathrm{Si} / \mathrm{SiO}_{2}{ }^{144-147}$ The semiconducting $\mathrm{MoS}_{2}$ exhibits a thickness-dependent band gap, ranging from $1.3 \mathrm{eV}$ for bulk $\mathrm{MoS}_{2}$ to $1.9 \mathrm{eV}$ for isolated monolayers, ${ }^{148}$ good conductivity and fast electron transfer. $\mathrm{MoS}_{2}$ is currently considered as one of the most preferable materials for chemical sensing applications, predominantly due to available edges that facilitate electron transfer. ${ }^{3,} 149$ Moreover, it can be easily produced in large scale and dispersed in numerous solvents to obtain desired structures. ${ }^{137,150}$ Layered tungsten disulphide $\left(\mathrm{WS}_{2}\right)$ nanosheets are one among the newly emerging TMDs, which consist of S-W-S sandwich structures. $\mathrm{WS}_{2}$ nanosheets have been the focus of intense research effort as catalyst, semiconducting material for field- 
effect transistors, active material in lithium-ion battery, etc. The ambipolar $\mathrm{WSe}_{2}$, has a similar trigonal lattice and a $1.6 \mathrm{eV}$ band gap (in the monolayer). Finally $\mathrm{ReS}_{2}, \mathrm{ReSe}_{2}$ and $\mathrm{PtSe}_{2}$ are recently explored TMDs, characterized by a highly distorted triclinic structure which confers to them extremely anisotropic optical, mechanical and electrical properties. ${ }^{151}$ Mono and few-layer $\mathrm{ReS}_{2}$ and $\mathrm{ReSe}_{2}$ can be obtained by mechanical exfoliation and CVD. ${ }^{152}$

Similar in structure to TMDs, $\mathrm{SnS}_{2}$ is a layered material possessing a triclinic structure which can be exfoliated in mono and few-layers with semiconducting properties. ${ }^{135}$ Compared to TMDs, $\mathrm{SnS}_{2}$ exhibits a higher electronegativity, which can potentially enhance the absorption sites and the sensing capabilities.

Among the emerging 2DMs black phosphorus (BP) has been recently re-discovered as a 2DM under the commonly used name of phosphorene. This material, which is, in analogy of graphene, a single honeycomb layer of the layered phosphorus allotrope can be obtained via mechanical or liquid exfoliation and presents remarkable electrical properties of tuneable direct band gap and high mobility. ${ }^{153}$ BP exhibits relatively low energy band gap, which depends on the number of layers and ranges from $0.3-1.5 \mathrm{eV} .{ }^{154,155} \mathrm{BP}$ exhibits fascinating optical and electronic properties such as size dependent optical response or anisotropic electrical conductivity, which simplifies characterization and segregation methods and makes BP promising material for various electrical applications. ${ }^{156,157}$ BP has already found several applications as FETs, photodetectors, solar cells and gas sensors. ${ }^{158-161}$ However, its application in devices is limited by the very low material's stability when exposed to air or water. ${ }^{162}$ Such a negative characteristic can become an advantage when fabricating chemical sensors.

Hexagonal boron nitride $(\mathrm{hBN})$ is a synthetic polymorph of boron nitride with layered structure analogue to graphite. Similarly to graphene, $\mathrm{hBN} \mathrm{WS} \mathrm{W}_{2}$ sexhibits a two-dimensional honeycomb-like structure with strong covalent bonds in the plane. It is characterized by relatively high band gap ranging from 3.6 to $7.1 \mathrm{eV}$, which determines its insulating properties. ${ }^{163,164}$ This feature provides transparency in the visible and near-IR regions extending its application potential. ${ }^{165}$ The weakly bonded layers can be exfoliated down to monolayer hBN, which has electrical insulating properties, but also high thermal conductivity, mechanical strength, hardness and chemical stability. ${ }^{166}$ The production method strictly determines the $2 \mathrm{D} \mathrm{hBN}$ nanosheets crystallinity, structure and other physical and chemical properties. Multiple methods are exploited for its production including mechanical 
exfoliation, ${ }^{167,} 168$ liquid exfoliation ${ }^{137,}{ }^{169-171}$ and chemical vapour deposition. ${ }^{172-174}$ Features such as good thermal conductivity, low toxicity, high mechanical strength, atomically smooth surface makes $\mathrm{BN}$ good candidate for sensing applications.

The large family of 2DMs also includes early transition metal carbides/nitrides (MXenes). This novel class of 2DMs shows promising properties toward a range of applications. MXene nanosheets originates from the MAX phases which constitute a class of nitrides and carbides in which $\mathrm{M}$ is an early transition metal, $\mathrm{A}$ is an element from group 13 or 14 of the periodic table, $\mathrm{X}$ is either $\mathrm{C}$ and/or $\mathrm{N}$. The MXene layers are obtained from the MAX phase by the removal of the element $\mathrm{A}$, which is intercalated between MXenes layers of the crystal, by treatment with HF and further sonication. ${ }^{175}$ MXenes display good electronic conductivity and unique morphology making them suitable for energy storage, ${ }^{176}$ electrochemical capacitors ${ }^{177}$ and chemical sensors. ${ }^{178,179}$

In the field of sensing it is generally preferred to make use of non-covalent interactions between the sorbents, i.e. the active material, and the analyte to ensure a quick response and a fast recovery rate (i.e. real-time monitoring). The use of pristine 2DM nanosheets has some drawbacks. A major limitation is represented by the poor porosity, which limits the number available recognition sites. In particular, 2DM nanosheets produced via LPE exhibit a strong tendency to self-aggregate and form even micron-sized stacked structures. Moreover, nanosheets produced via LPE exhibit low charge carrier as well as low field-effect mobility.

Alongside the capacity of pristine 2DMs to detect various analytes, extensive efforts have been devoted towards the development of 2DMs-based sensors through the non-covalent functionalization of the 2DMs with both inorganic and organic moieties which act as spacers/pillars imposing a certain distance between adjacent sheets. This results in an enhanced porosity of 2DM-based composite which determines a greater sensitivity for the analyte of choice. Such an approach enables the tailoring of the properties of 2DM-based sensors, which could preserve many of the unique characteristics of the individual 2DM sheets and benefit from the presence of (in)organic moieties. Such moieties, besides acting as separators, can by design incorporate the receptor of the analyte of choice, endowing highest selectivity in the recognition and sensing process. In this context, the fabrication of 2DMbased sensors through non-covalent interactions between individual 2DM sheets is extremely appealing, as it could result in the structures exhibiting a remarkable enhancement of sensitivity towards specific analytes. 


\section{Applications in gas sensing}

A gas sensor is a device that can detect the presence and quantify the concentration amount of a specific gas in the atmosphere such as water vapour (humidity), organic vapours and hazardous gases. ${ }^{180}$ Gas sensors have attracted a strong interest and are widely employed in environmental monitoring and emission control, ${ }^{181}$ personal and military safety, ${ }^{182}$ production control in agriculture and industry and medical diagnostics. ${ }^{183-185}$ Among variety of materials used for gas sensing devices, metal oxides have been largely considered for their high sensitivity and low costs; however, their crucial drawbacks as high temperature operation and large energy consumption, as well as poor selectivity, have hindered their practical application. ${ }^{186}$ Therefore, many efforts have been devoted in developing new gas sensors with high sensitivities and low operating temperatures. Numerous active materials have been considered including conducting polymers ${ }^{187}$ and carbon nanotubes (CNTs), ${ }^{188}$ which possess interesting low operating temperatures but suffer from long response and recovery time, poor stability, degradation and difficult processing.

In the last few years 2DMs are emerging as new materials for gas sensing by virtue of their unique properties, which promise to largely improve the sensitivity of the sensors. Among 2DMs, graphene is particularly appealing, because of its low resistivity and electrical noise. ${ }^{189}$ Such characteristics enable the detection of small changes in the intrinsic resistance resulting from the interaction of graphene with gaseous species. Alongside graphene, also its analogues such as TMDs $\left(\mathrm{MoS}_{2},{ }^{190-193} \mathrm{WS}_{2},{ }^{194,}{ }^{195} \mathrm{MoSe}_{2}\right.$ etc.), layered metal oxides $\left(\mathrm{MoO}_{3},{ }^{196} \mathrm{SnO}_{2}{ }^{197,}{ }^{198}\right)$, phosphorene, ${ }^{158}$ and h-BN ${ }^{199}$ etc. have also been employed for gas sensing by exploiting their thickness dependent characteristics and semiconducting properties. Indeed, the presence of a band gap that can be modulated by the interaction with gases is a tool that enables enhanced sensitivity. Furthermore, the semiconducting properties of these materials render them suitable for being integrated as active components in FETs, permitting low power consumption and miniaturized devices. ${ }^{199}$ In these devices, the adsorption of gases on the semiconducting material determines a change in their conductivity, which can be measured as a variation in the drain current. ${ }^{144}$ Other common gas sensing devices are represented by chemiresistors ${ }^{185}$ and chemi-capacitors, ${ }^{200}$ in which the sensing material is interposed between two electrodes, and the gas molecules adsorbed on the surface of the material induce a change in the resistance or capacitance, which can be directly quantified. 
The performances of the gas sensors are usually characterized by various figures of merit, with the most important being sensitivity, selectivity, stability, response, and recovery time as well as the costs, dimensions and flexibility. In this chapter we will define the response $\mathrm{R}(\%)$ of a given device as the ratio between the difference in resistance (or another output) when it is operating in the presence or in the absence of the sensed gas:

$R(\%)=\frac{R(i)-R_{0}}{R_{0}} * 100 \quad$ (eq. 1$)$

In this part of review we will provide an overview over the gas sensing mechanism for devices containing active 2DMs, and we will present the most interesting and up-to-date gas sensing applications of graphene, its derivatives, TMDs and other 2DMs.

\subsection{Graphene-based gas sensing}

\subsubsection{Gas sensing with pristine graphene}

Graphene sheet exhibits two exposed surfaces without a bulk separation, thus it has all its atoms exposed to the environment; such a structure makes it an ideal building block for the detection of gas molecules. ${ }^{201}$ Furthermore, the high variability of its resistance and low electrical noise of some devices makes it possible to detect even extremely small changes in the number of carriers generated by the interaction with gas molecules. ${ }^{124}$ The most frequent detection mechanism of graphene, as well as many other 2DMs, is based on charge transfer process, in which the sensing material and the gas molecules behave as charge donors or acceptors. $^{202}$ In particular, Leenaerts et $a .^{203}$ used density functional theory (DFT) calculations to demonstrate that gas molecules adsorbed on the surface of graphene act as a dopant changing the carrier density of graphene. The interaction energies of $\mathrm{H}_{2} \mathrm{O}, \mathrm{CO}, \mathrm{NO}$, $\mathrm{NO}_{2}$ and $\mathrm{NH}_{3}$ with graphene were calculated, showing that $\mathrm{H}_{2} \mathrm{O}$ and $\mathrm{NO}_{2}$ molecules behave as strong electron acceptors for graphene, thereby resulting in a decrease of charge carriers in the latter, while $\mathrm{NH}_{3}, \mathrm{NO}$ and $\mathrm{CO}$ behave as electron donor enhancing the concentration of charge carriers. Upon exposure of the graphene surface to air or to an inert gas the sensed gas molecules are promptly desorbed and the initial conductivity of graphene is recovered.

The first application of graphene as a gas sensor was reported in 2007 by Novoselov et al. ${ }^{201}$ A micromechanically exfoliated graphene flake supported on $\mathrm{Si} / \mathrm{SiO}_{2}$ substrate was firstly etched in a bar shape and patterned with top gold contacts exploiting Electron-Beam 
lithography (EBL) (Figure 2a) and later exposed to $\mathrm{H}_{2} \mathrm{O}, \mathrm{NO}_{2}, \mathrm{NH}_{3}$ and $\mathrm{CO}$ gases. It was shown that the presence of gas molecules causes a doping in the graphene changing the concentration of charge carriers. In particular, it was demonstrated that $\mathrm{H}_{2} \mathrm{O}$ and $\mathrm{NO}_{2}$ decrease the charge carrier concentration while an increase of carrier concentration was observed when $\mathrm{NH}_{3}$ or $\mathrm{CO}$ were employed (Figure 2b). Furthermore, exploiting the Hall resistivity, changes in the number of carriers of just a single electron were investigated in the vicinity of the Dirac point. In this way, by exposing the device to very diluted $\mathrm{NO}_{2}$ gas it was possible to sense the adsorption of a single $\mathrm{NO}_{2}$ molecule. In fact, it can be seen that the Hall resistivity varies in a step-like manner, because each $\mathrm{NO}_{2}$ molecule adsorbed/desorbed produces a variation of one electron in the number of carriers (Figure 2c). This outstanding performance was attributed to the high carrier mobility and extremely low noise of the so patterned graphene device.

In 2009 Dan et al. ${ }^{204}$ demonstrated that the outstanding sensing performances of Novoselov's device could not only be ascribed by the use of pristine graphene, whose inert surface is not optimal for adsorbing molecules, but it can also be attributed to the lithographic process employed for device fabrication. Towards this end, a miniaturized FET based on mechanically exfoliated graphene on $\mathrm{Si} / \mathrm{SiO}_{2}$ substrate was fabricated by EBL and used to detect various gas vapours, including $\mathrm{H}_{2} \mathrm{O}$, nonanal, octanoic acid, and trimethylamine down to the ppm level. It was claimed that the sensitivity of the device was enhanced by the presence of a contamination layer on the graphene surface induced by EBL process. This layer consists of residues such as the photoresist, acting as an absorbent layer that concentrates analyte molecules onto the graphene surface. In fact once this layer was removed by $\mathrm{Ar} / \mathrm{H}_{2}$ etching, the device became almost completely insensitive to the analysed vapours. A similar device based on mechanically exfoliated graphene patterned trough EBL was developed by Ko et al. to detect $\mathrm{NO}_{2}$, with a response of $9 \%$ to $100 \mathrm{ppm}$ of $\mathrm{NO}_{2}{ }^{80}$

EBL was also employed by Balandin et al. ${ }^{205}$ to fabricate a FET that can selectively detect different organic vapours through analysis of the low frequency noise of the device. In particular, it was shown that the gas molecules act as trap centres, leading to fluctuation in the carrier concentration and thus noise. In addition, the different kinetics of adsorption/desorption of the different vapours lead to different characteristic noises and made it possible to selectively distinguish the presence of tetrahydrofuran (THF), methanol $(\mathrm{MeOH})$, acetonitrile $(\mathrm{MeCN})$ and chloroform in the environment. 

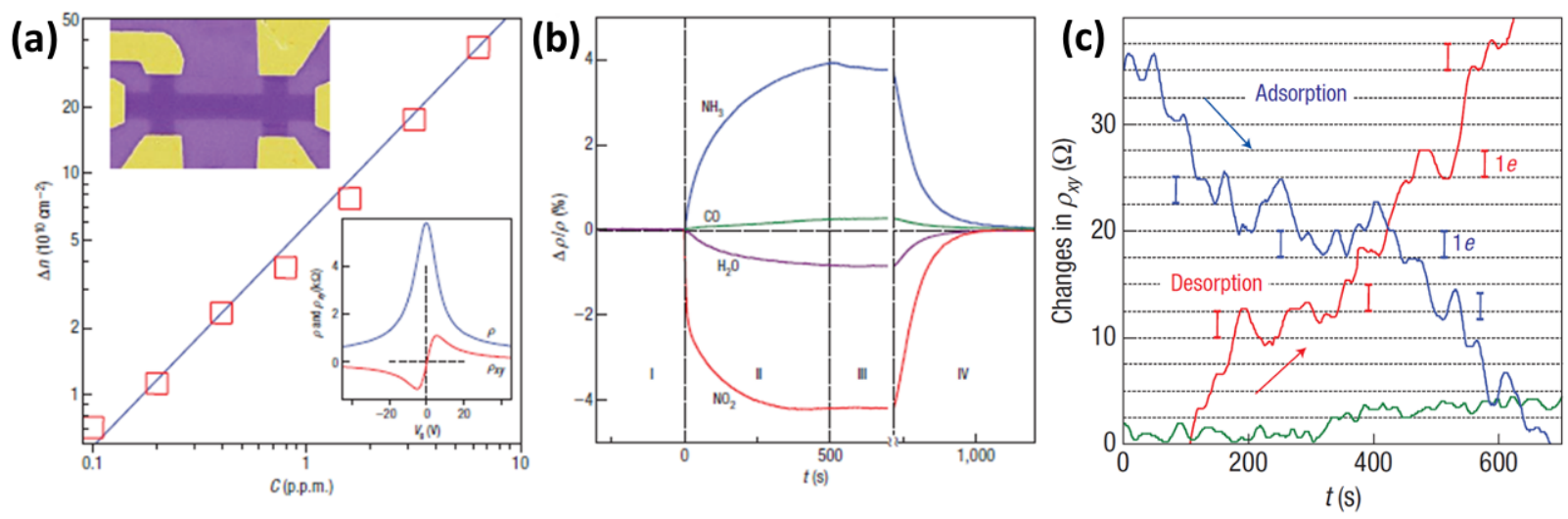

Figure 2. (a) Dependence of the concentration of charge carriers $(\Delta \mathrm{n})$ in single-layer graphene when exposed to different concentrations (C) of $\mathrm{NO}_{2}$. Upper inset: Scanning electron microscope (SEM) image in false colour of this device (the device is $1 \mu \mathrm{m}$ wide). Lower inset: Characterization of the graphene device by using the electrical field-effect. (b) Changes in resistivity caused by graphene's exposure to $1 \mathrm{ppm}$ of various gases highlighting the positive or negative doping effects. (c) Changes in Hall resistivity observed near the Dirac point during adsorption (blue curve) and desorption (red curve) of strongly diluted $\mathrm{NO}_{2}$. The green curve is a reference of the same device exposed to pure He. The grid lines correspond to changes in $\rho_{x y}$ caused by adding one electron charge. Figure adapted from Ref. ${ }^{201}$ with permission from Springer Nature.

Graphene obtained by mechanical exfoliation was employed also by Yoon et al. ${ }^{206}$ as a sensor of $\mathrm{CO}_{2}$. In order to minimize the number of residues resulting from the traditional "scotch tape" cleavage method and from the EBL process, a monolayer of graphene was exfoliated from the bulk by employing a polydimethylsiloxane (PDMS) stamp and transferred on top of gold electrodes pre-patterned on a $\mathrm{Si} / \mathrm{SiO}_{2}$. Such a device showed moderately fast $(\sim 10 \mathrm{~s})$, reversible, high and linear response to a concentration of $\mathrm{CO}_{2}$ ranging between 10 and $100 \mathrm{ppm}$ at room temperature. A similar device based on mechanically exfoliated graphene was developed by Ko et al. to detect $\mathrm{NO}_{2}$, with a response of $9 \%$ to $100 \mathrm{ppm}$ of $\mathrm{NO}_{2}{ }^{80}$

Larger and high-quality graphene sheets can be obtained by chemical vapour deposition: ${ }^{121}$ such graphene type has been used to detect $\mathrm{NO}_{2},{ }^{207-210} \mathrm{NH}_{3},{ }^{210-212} \mathrm{CO}_{2}$ and humidity. ${ }^{213} \mathrm{CVD}$ graphene devices showed in fact good sensitivity towards the detected gases, yet it suffered from difficult desorption of the sensed gases and exhibited slow and incomplete recovery at low temperatures. CVD graphene monolayers transferred onto $\mathrm{Si} / \mathrm{SiO}_{2}$ substrates were used 
by Gautam et al. to sense $\mathrm{NH}_{3}, \mathrm{CH}_{4}$ and $\mathrm{H}_{2} .{ }^{211}$ The best performance of the device in terms of sensitivity was observed when operating at $150-200{ }^{\circ} \mathrm{C}$, yet the use of such extreme experimental conditions was not accompanied by an improvement in the response time. Choi et al. ${ }^{209}$ realized a transparent and flexible $\mathrm{NO}_{2}$ detector, in which single layer graphene (SLG) was employed as a sensing active component and bi-layer graphene (BLG) was used as an internal heater: by applying an electrical power of $1.7 \mathrm{~W}$ to the device it was possible to heat the device itself up to $200{ }^{\circ} \mathrm{C}$ for joule effect. Such a device showed a response of $39 \%$ to $40 \mathrm{ppm}$ of $\mathrm{NO}_{2}$ and the presence of the heaters was found to accelerate the recovery time of the device reaching values of a few seconds. Kim et al. ${ }^{210}$ in 2015 presented a similar selfheated, transparent, flexible $\mathrm{NO}_{2}$ sensor. CVD SLG was patterned on the $\mathrm{Cu}$ foil to obtain a conductive channel of $5 \mu \mathrm{m}$ width between two graphene electrodes and then transferred onto a flexible Polyimide (PI) substrate (Figure 3a). Also in this case, the SLG micro channel was self-heated up to $73{ }^{\circ} \mathrm{C}$ by applying a bias of $60 \mathrm{~V}$, yielding an increase in the sensitivity and recovery time of the device $\left(\Delta \mathrm{R} / \mathrm{R}_{0}=12 \%\right.$ and recovery time of $82 \mathrm{~s}$ to $5 \mathrm{ppm}$ of $\mathrm{NO}_{2}$ at 60 V) (Figure 3b-c). This device exhibited also good selectivity, reversibility and durability even under mechanical bending and negligible influence of humidity. Yavari et al. ${ }^{212}$ in 2011 realized a sensor based on unconventional CVD graphene. In particular, graphene foam was produced by growing CVD graphene onto a porous nickel template, which was dissolved afterwards to obtain the self-standing foam. After contacting the foam with two electrodes a $30 \%$ response to $1000 \mathrm{ppm}$ of $\mathrm{NH}_{3}$ was measured, yet the response and the recovery time were both found being slow $(\sim 1000 \mathrm{~s})$.
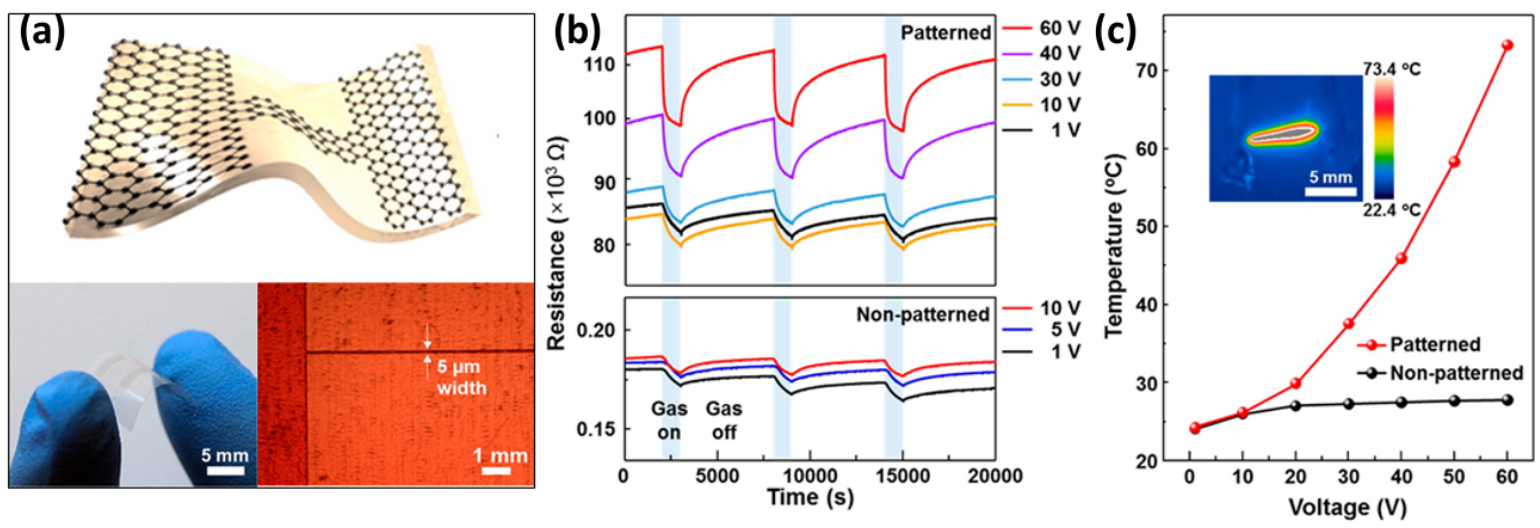

Figure 3. (a) Scheme (top) and photograph (bottom left) of the all-graphene flexible, transparent gas sensor and micrograph of patterned graphene (bottom right). (b) Response curves, i.e. resistance changes, recorded in patterned and non-patterned graphene sensors when exposed to three pulses of $5 \mathrm{ppm}$ of $\mathrm{NO}_{2}$. (c) Thermographic image and thermal 
characteristics at different bias voltages. (Figure adapted from Ref. ${ }^{210}$ with permission from the American Chemical Society)

The sensing performance can be tailored by the choice of the substrate in epitaxially grown graphene. ${ }^{214}$ In fact $\mathrm{SiC}$ possesses a wide number of different polymorphs and each one of them, as well as diverse crystal faces, can exhibit doping effects on graphene. ${ }^{215}$ As an example Pearce et al. ${ }^{216}$ realised a $\mathrm{NO}_{2}$ sensor based on epitaxially grown SLG on the atomically flat $\mathrm{Si}$ face of $4 \mathrm{H}-\mathrm{SiC}$. This sensor displayed an extremely high response to 2.5 ppm of $\mathrm{NO}_{2}\left(\Delta \mathrm{R} / \mathrm{R}_{0} \approx 120 \%\right)$. Interestingly, the response switched from $n$-type to $p$-type at increasing $\mathrm{NO}_{2}$ concentration because of the intrinsic $n$-doped nature of graphene grown on $4 \mathrm{H}-\mathrm{SiC}$. This behaviour was also demonstrated by Nomani et al. ${ }^{214}$ In their work graphene grown on $\mathrm{C}$ face of $6 \mathrm{H}-\mathrm{SiC}$ and on $\mathrm{Si}$ face had opposite behaviour when exposed to $18 \mathrm{ppm}$ of $\mathrm{NO}_{2}$ : the one grown on $\mathrm{C}$ face was $n$-type and exhibited a $4.5 \%$ increase of the conductance, while the other was $p$-type and exhibited a $10 \%$ decrease of the conductance.

In order to enhance the performance of graphene-based gas sensor a promising solution consists in introducing defects and functional groups. In fact, the absence of dangling groups on graphene's surface renders it inert to the chemisorption of gas molecules. For example, the ozone treatment of graphene sheets is a viable approach to introduce oxygen functional groups uniformly on the surface. Such defects can act as recognition sites and promote the interaction with gaseous molecules through hydrogen bonds and van der Waals forces. ${ }^{217}$ Chung et al. ${ }^{208}$ treated CVD SLG with ozone and demonstrated that the so-treated graphene features better performances in terms of sensitivity and response time for detecting $\mathrm{NO}_{2}$ compared to pristine CVD graphene. Along the same line, Masel et al. ${ }^{218}$ proved that the CVD polycrystalline graphene was much more sensitive to various organic vapours than monocrystalline pristine graphene because of the presence of structural defects.

\subsubsection{Gas sensing with graphene oxide and reduced graphene oxide}

The key role played by oxygen containing functional groups in graphene oxide is perfectly highlighted by its sensing capabilities. The sensing performances of GO can be easily tailored by varying the amount of it oxygen containing functional groups via reduction (thermal or chemical) towards rGO. These unique characteristics make pristine and reduced GO the most studied platforms for gas sensing. ${ }^{124}$ Numerous articles on GO, ${ }^{200,} 219 \mathrm{rGO}^{220,} 221$ and 
functionalized GO (fGO) $)^{222,223}$ based gas sensors have been published. The first application of rGO as gas sensor was developed by Robinson et al. in $2008 .^{73} \mathrm{GO}$ was spin-coated on the top of a $\mathrm{Si} / \mathrm{SiO}_{2}$ substrate and $\mathrm{Ti} / \mathrm{Au}$ pads were evaporated as top electrodes (Figure $4 \mathrm{a}$ ). The device was exposed to hydrazine vapours at $100{ }^{\circ} \mathrm{C}$ to trigger the reduction of GO to rGO. Figure $4 \mathrm{~b}$ portrays the response of the device when exposed to pulses of acetone vapours for 5 s. The response (e.g. electrical) of the device varies depending on the duration of the reduction treatment. In particular, it is possible to observe two response components: a fast response, which increases with the reduction time due to the decrease of the number of oxygen functional groups, and a slow response, which decreases with increasing reduction time. It was explained that the fast response is the result of the gas adsorption onto lowenergy binding sites, such as $\mathrm{sp}^{2}$-bonded carbon, while the slow response occurs from the interaction of gases with higher-energy binding sites, such as structural defects, and oxygen bearing functional groups. Furthermore, the electrical noise of the device can be pushed to the limit of the thermal noise (Figure 4c) for very thin films and long reduction treatment, being much lower of CNTs network. Thanks to the low electrical noise the limit of detection of various vapours was found being extremely low. For example, it was possible to sense $70 \mathrm{ppb}$ of $\mathrm{HCN}$ and $5 \mathrm{ppb}$ of dimethyl methylphosphonate (DMMP).

A flexible all printed gas sensor based on rGO reduced with ascorbic acid was realized by Manohar et al. ${ }^{224}$ This was done by preparing a dispersion of rGO in water in presence of a surfactant, followed by its ink-jet printing onto a PET substrate. The device displayed a response with a detection limit on the tens of ppm range; such a response is positive, i.e. displaying increasing resistances, for $\mathrm{NH}_{3}$, dichloromethane and various alcohols, whereas it was found providing negative response for $\mathrm{Cl}_{2}$ and $\mathrm{NO}_{2}$. Such phenomenon has been attributed to the electron withdrawing character of $\mathrm{Cl}_{2}$ and $\mathrm{NO}_{2}$, which enhance the conductivity of semiconducting $p$-type $\mathrm{rGO}^{212}$ In another case, ${ }^{225}$ a $\mathrm{NO}_{2}$ gas sensor was fabricated by reducing and patterning via laser irradiation the GO on various flexible substrates. The device exhibited a good sensitivity, which could allow the detection of 20 ppm of $\mathrm{NO}_{2}$, with complete, yet slow recovery. Another chemiresistive $\mathrm{NO}_{2}$ sensor was developed by Prezioso et al. ${ }^{219}$ by using pristine GO; this device yielded the best performance when heated at $150{ }^{\circ} \mathrm{C}$, with a response of $60 \%$ to $5 \mathrm{ppm}$ of $\mathrm{NO}_{2}$, yet accompanied by the response and recovery time close to an hour. In another work $^{226}$ the authors improved this $\mathrm{GO}$ based $\mathrm{NO}_{2}$ sensor which reached a detection limit of just $20 \mathrm{ppb}$. Furthermore, this sensor had a response to $\mathrm{NO}_{2}$ unaffected by the humidity level and was able 
to detect also the presence of ethanol, acetone and ammonia. Sinitskii et al. ${ }^{222}$ fabricated a highly selective gas sensor able to discern three different alcohols such as methanol, ethanol and isopropanol with a high success rate by using a 20-channels array integrated sensor of thermally reduced $\mathrm{GO}$, in which each channel had a unique response due to the structural/morphological irregularity of the rGO film.

(a)

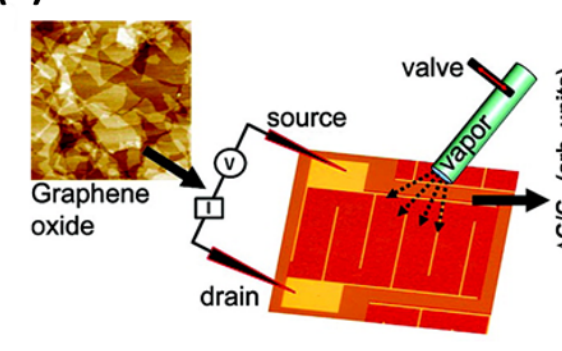

(b)

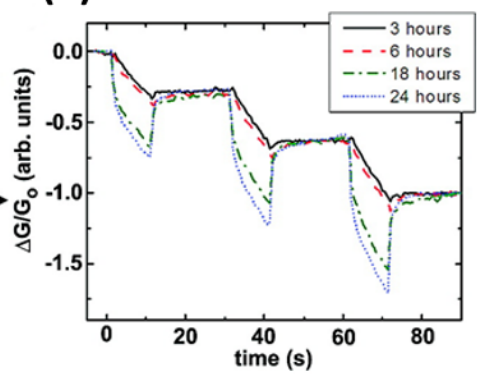

(c)

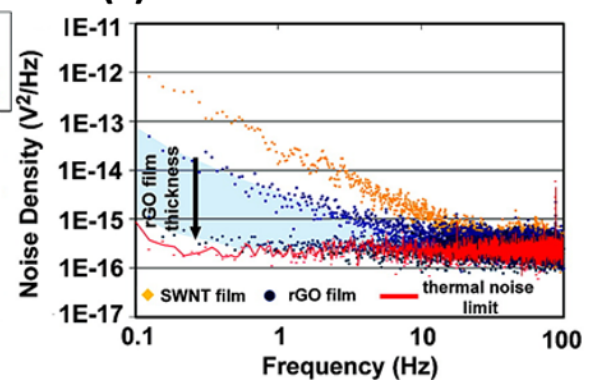

Figure 4. (a) AFM image of the GO film and scheme of the device. (b) Plot of the response to $5 \mathrm{~s}$ pulses of acetone for GO devices reduced with hydrazine for 3, 6, 18, and $24 \mathrm{~h}$. (c) Noise density spectrum for SWNT and rGO devices. Adapted from Ref. ${ }^{73}$ with permission from American Chemical Society.

Among the various gas sensing applications, GO and rGO have been extensively used also as humidity sensors. ${ }^{227-230}$ Humidity is the presence of water vapour in air and is normally measured as relative humidity $(\mathrm{RH})$ which is the ratio between the partial pressure of water and the equilibrium vapour pressure at a given temperature. It is worth reminding that having a good control over the humidity in the environment is key for numerous industrial and technological processes and most importantly to ensure the comfort of live beings, improving the quality of life in living and working places

The presence of numerous oxygen-bearing functional groups as hydroxyl, epoxy and carboxylic groups renders GO's surface highly hydrophilic, thus being a perfect candidate for sensing moisture. ${ }^{71}$ Ruoff et al. ${ }^{231}$ in 2008 demonstrated that the interaction of water molecules with these functional groups of GO determines changes in the conductivity of GO; the authors therefore concluded that highly reduced GO is not suitable for humidity sensing. Water interacts with GO mainly by increasing its conductivity because of the formation of charge carriers as hydronium and hydroxide ions as demonstrated by Yao et al. in $2012 .{ }^{230}$ In their work, GO was drop-cast on an interdigitated electrode (IDE); when a potentials $<2 \mathrm{~V}$ 
was applied a 10-fold change in the GO's conductivity was observed upon varying the RH between 15 and $95 \%$. Such a conductivity change was found being even larger when higher potentials were applied and has been attributed to the favoured ionization of the water molecules. Similarly Borini et $a .^{227}$ spray-cast a solution of GO on top of interdigitated electrodes (IDE) screen-printed on a flexible and transparent polyacrylonitrile (PAN) substrate. Thin films of spray-coated GO can be essentially reduced to a simple resistorcapacitor (RC) equivalent circuit in which a resistor and a capacitor are in parallel. It was found that resistance displayed an impressive exponential dependence from $\mathrm{RH}$, with a 10fold decrease when increasing the RH from 40 to $70 \%$ (Figure 5a). Furthermore, this device showed an ultra-fast response and recovery to pulses of humid air of just $20 \mathrm{~ms}$ (Figure $5 \mathrm{~b}$ ). Another GO based flexible humidity sensor was prepared by Guo et al. ${ }^{220}$ via spin-coating on PET substrates. The GO film was micro-patterned by using two-beam-laser interference to create alternating lines of $\mathrm{GO}$ and $\mathrm{rGO}$. By tuning the laser power, it was possible to control the conductivity as well as the response/recovery time. The best results were obtained at 0.2 $\mathrm{W}$ laser power, with a response and recovery time of a few seconds and a change in the resistance of 1 order of magnitude between 11 and $95 \%$ RH. In a very recent work, Tai et al. ${ }^{229}$ underlined the effect of the degree of reduction on the humidity sensing properties of GO. While GO showed a decrease in the resistance for increasing humidity, rGO displayed the opposite behaviour. By annealing $\mathrm{GO}$ at $150{ }^{\circ} \mathrm{C}$ for different times, integrating the soobtained material in humidity sensors, and studying them with electrochemical impedance spectroscopy (EIS) they concluded that the resulting resistance of macroscopic film of GO is essentially composed by two resistances in series: an intrinsic resistance within the flake $\left(\mathrm{R}_{\text {int }}\right)$ and a junction resistance between flakes $\left(\mathrm{R}_{\text {junct }}\right)$. Both these terms are humidity dependent; in particular, the first can be expressed as two resistors in parallel, one in-plate resistance dependent only on the reduction grade and the ionic resistance, which decreases with the increasing of humidity. The second term $\mathrm{R}_{\text {junct }}$ can also be expressed as two elements in parallel, a capacitor $\left(\mathrm{C}_{\mathrm{EDL}}\right)$ and a resistor $\left(\mathrm{R}_{\mathrm{Ohm} \text {-contanct }}\right)$ both humidity dependent: when humidity increases and the GO film adsorbs water molecules the distance between flakes increases, thus yielding to an increase in both the values $\mathrm{C}_{\mathrm{EDL}}$ and $\mathrm{R}_{\text {Ohm-contact. }}$ As a result, with the increasing of humidity, $\mathrm{R}_{\text {int }}$ decreases (negative response) and $\mathrm{R}_{\text {junct }}$ increases (positive response). For GO $\mathrm{R}_{\text {int }}$ is greater than $\mathrm{R}_{j u n c t}$, thus leading to a negative response to the increased humidity, while for $\mathrm{rGO} \mathrm{R}_{\text {int }}<\mathrm{R}_{\text {junct }}$ thus yielding to a positive response to the increased humidity (Figure 5d). 


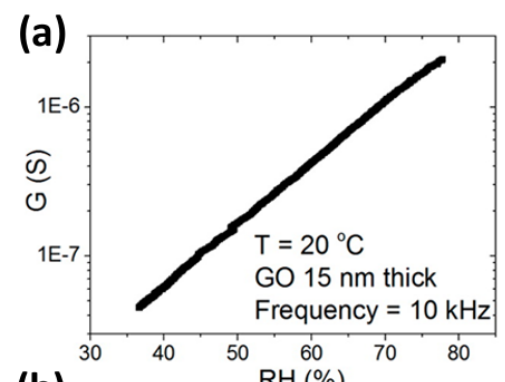

(c)
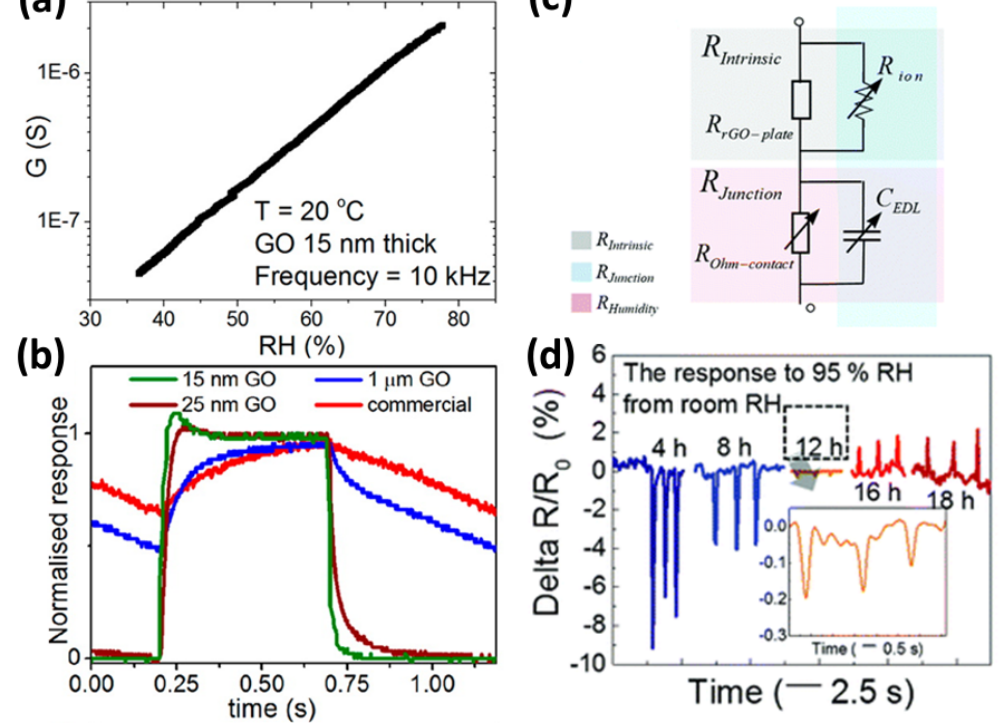

Figure 5. (a) Conductance dependence of an ultrathin GO film to the RH of the environment. (b) Normalized response of the different sensors to a modulated humid air flow at $1 \mathrm{~Hz}$. Adapted from Ref. ${ }^{227}$ with permission of American Chemical Society. (c) Equivalent electrical circuitry model of rGO representing intrinsic and junction-dependent resistance. (d) Response of rGO films with different thermal reduction times to humidity pulses. Adapted from Ref. ${ }^{229}$ with permission from the Royal Society of Chemistry.

In order to target more specific types of gases, GO and rGO have been functionalized with (macro)molecules bearing specific functional groups acting as selective active sites. The molecules can be chemisorbed on the surface of graphene or physisorbed on its surface. For example Al-Mashat et al. ${ }^{232}$ reported an hydrogen sensor based on an assembly of polyaniline (PANI) adsorbed on the rGO surface. The material was synthetized via ultrasonication of an alcoholic mixture of $\mathrm{rGO}$, aniline and the polymerization initiators, which promoted the polymerization of PANI selectively on the rGO surface. Then, the mixture was spray coated on a quartz substrate with gold IDE. This functionalized rGO showed a better sensitivity to $\mathrm{H}_{2}$ compared to pure rGO and PANI with a $16.6 \%$ response to $1 \% \mathrm{H}_{2}$ gas. The improved sensitivity was attributed to the higher porosity of the hybrid structure in respect to the bare PANI. In another case, an assembly PANI-rGO was used for $\mathrm{NH}_{3}$ sensing ${ }^{233}$. Shi et al. ${ }^{234}$ fabricated a $\mathrm{NO}_{2}$ chemresistive-type gas sensor based on sulfonated rGO (S-rGO) and rGO functionalized with ethylenediamine (EDA-rGO). In particular, S-rGO displayed a response to $\mathrm{NO}_{2} 10$ times greater of $\mathrm{rGO}$ and 3 times larger than EDA-rGO. Furthermore, the S-rGO exhibited also faster response and recovery and good selectivity towards $\mathrm{NO}_{2}$. The superior sensitivity of the S-rGO sensors can be attributed 
to the electron withdrawing characteristics of sulfophenyl groups, which enhance the hole doping in $p$-type rGO. After the absorption of $\mathrm{NO}_{2}$ the $p$-doping is further boosted therefore harnessing the material's conductivity. Hu et al. ${ }^{223}$ developed a DMMP sensor based on rGO functionalized with p-phenylenediamine, which was deposited from solution on a IDE. The response of this device to DMMP vapours was much higher of the one of hydrazine reduced GO $\left(\Delta \mathrm{R} / \mathrm{R}_{0}\right.$ to $30 \mathrm{ppm} \mathrm{DMMP}=11 \%$ for $\mathrm{f}-\mathrm{rGO}$ and $1.5 \%$ for $\left.\mathrm{rGO}\right)$; however, the response and recovery times were on the scale of several minutes. The chemical functionalization of GO can be used to build sensor arrays in order to recognize different gases. In a very recent work Jelinek et al. ${ }^{200}$ assembled a capacitive-type porous graphene oxide (pGO) vapour sensor in which the GO was deposited on a IDE through a freeze-drying technique in order to maximize the area exposed to the gas. In parallel the GO was also chemically functionalized with aniline (phenyl-GO), dodecylamine (dodecyl-GO) and ethanolamine (ethanol-GO) by exploiting the amidation reaction between the amines and the carboxylic groups present on the GO surface - yet, the functionalization of epoxy groups may also occur. These three differently functionalized GOs were deposited on IDE similarly to pGO and by combining them in an array it was possible to selectively detect the presence of water, $\mathrm{NH}_{3}$, toluene, EtOH, phenol and cyclohexane (Figure 6). Additionally, the sensitivities of these devices towards the target gases were high, with high reproducibility and both response and recovery times on the time scale of few seconds.

The doping of GO and rGO has also been used to improve the performance of humidity sensors. Rathi and $\mathrm{Pal}^{228}$ demonstrated that GO doped with Li and B have higher sensitivity to the humidity compared to GO. The Li-GO and B-GO were prepared through reaction of $\mathrm{GO}$ with $\mathrm{LiOH}$ and boric acid, then these doped GO water solutions were drop-casted on a glass with two copper electrodes on top. The Li doped GO in particular showed a response to the humidity 4 times higher than un-doped GO. Also in this case the higher performances can be attributed to the increased $p$-doping of Li-GO. 

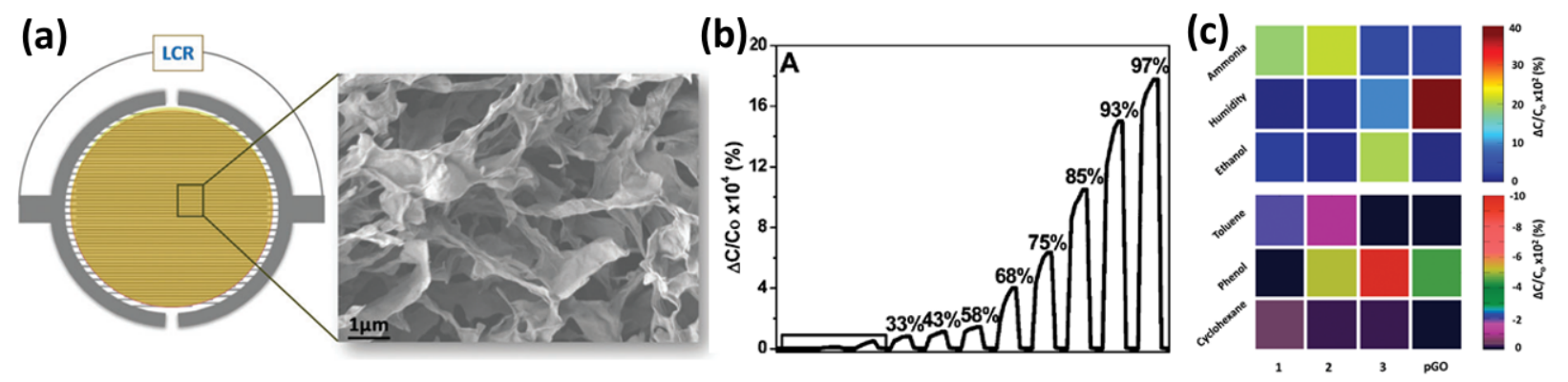

Figure 6. (a) Schematic structure of the device and SEM image of the p-GO. (b) Response of the p-GO device to different values of humidity (RH \%). (c) Array-based colour code identification of different vapours (180 ppm) using the functionalized pGO capacitive sensor: phenyl-GO (1); dodecyl-GO (2); ethanol-GO (3). The colours indicated in the diagram correspond to the percentage capacitance response, according to the colour keys shown on the right. Adapted from Ref. ${ }^{200}$ with permission from Royal Society of Chemistry.

\subsubsection{Gas sensing with graphene based composites}

Hybrid composites, made by combining graphene with functional materials (such as metals, metal oxides, polymers, etc.) can exhibit significantly increased porosity compared to films of pristine 2DMs, thus resulting in increased accessible surface area. Furthermore, such approach harnesses the sensing performances of $2 \mathrm{DMs}$ due to the combination of different functionalities and their synergistic effects. ${ }^{75}$ Metallic nanoparticles (NPs) and films (especially those of noble metals) can enhance the sensitivity and selectivity due to specific catalytic effects. $^{235}$

For example, it is well-known that the metals such as palladium and platinum exhibit a catalytic activity towards the adsorption of hydrogen. ${ }^{236}$ With this in mind, palladium and platinum-graphene composites have been extensively explored to target $\mathrm{H}_{2}$ gas; ${ }^{182}$, 235, 237-241 hydrogen is colourless and odourless but mixed with air forms an explosive mixture; thus, its detection is of paramount importance for safety. Kaniyoor et al. ${ }^{235}$ produced a hydrogen sensor based on GO decorated with Pt NPs: the Pt NPs were grown in situ on the GO surface and then the so decorated GO was drop-casted on an alumina substrate with pre-patterned $\mathrm{Cu}$ electrodes. This device showed a $16 \%$ increased electrical resistance when exposed to $4 \%$ $\mathrm{H}_{2}$ yet accompanied with a slow responsivity. Similarly, Wu et al. ${ }^{237}$ fabricated an $\mathrm{H}_{2}$ sensor based on a Pd thin film ( $1 \mathrm{~nm})$ evaporated over CVD graphene with $12 \%$ sensitivity towards 
the exposure to $1 \% \mathrm{H}_{2}$. Chung et al. ${ }^{238}$ realized a flexible hydrogen sensor composed by CVD graphene decorated with Pd NPs exhibiting an impressive sensitivity of $30 \%$ to $1 \%$ $\mathrm{H}_{2}$. Reduced GO decorated with Pd NPs has also been used to sense NO gas for medical applications by Li et al. ${ }^{185}$ The rGO with physically absorbed Pd NPs on its surface was deposited on CVD graphene-coated Ni electrodes (

Figure 7a). This device exhibited high sensitivity with an impressive limit of detection of 2 $\mathrm{ppb}$, being notably higher than the reference device assembled without decorated rGO or without graphene-coated electrodes (

Figure 7b). In this case Pd NPs may act as an absorption site of NO molecules, promoting the donation of electrons from NO to rGO. Furthermore, the interaction of the hybrid with NO can lower the Schottky barriers between rGO and Pd NPs leading to a further increase in the conductance.

Interestingly, the modification of rGO with functional groups capable of boosting the $p$ doping can be combined with the catalytic activity of metal NPs. For example Huang et al. ${ }^{242}$ assembled a flexible, all-printed $\mathrm{NO}_{2}$ sensor based on sulfonated $\mathrm{rGO} / \mathrm{Ag}$ NPs. The $\mathrm{rGO}$ was firstly sulfonated and then Ag NPs were grown in situ on the rGO surface via hydrazine reduction of $\mathrm{AgNO}_{3}($

Figure 7c). Then Ag electrodes and the $\mathrm{rGO} / \mathrm{Ag}$ NPs based ink were printed on a PI substrate. This sensor displayed a $74.6 \%$ fast response to $50 \mathrm{ppm}$ of $\mathrm{NO}_{2}$. Besides, the sensor was quite robust: it exhibited a good stability over time and tolerance to humidity (

Figure 7d). The sensor could also detect $\mathrm{NH}_{3}$, with a similar, yet a negative response. 

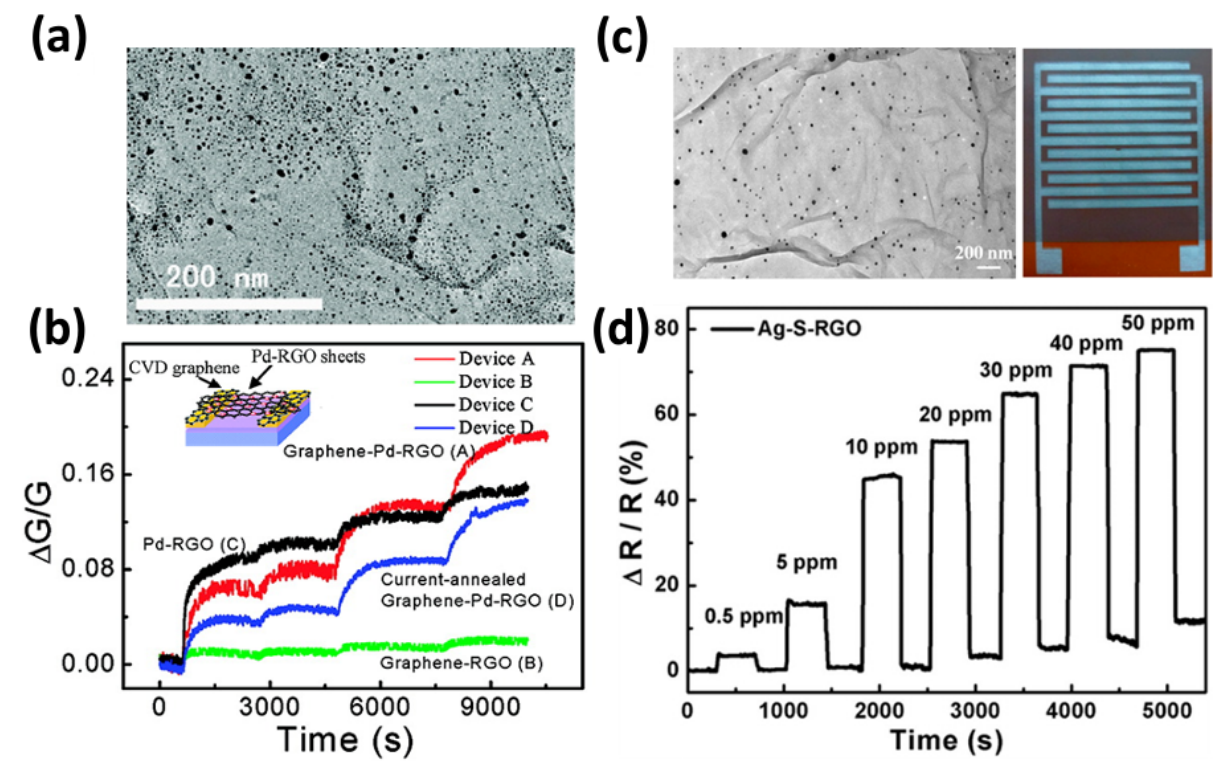

Figure 7. (a) Transmission Electron Microscopy (TEM) images of Pd-rGO sheets. (b) Relative changes in conductance of the various devices versus time when exposed to 1,10 , 60 , and $120 \mathrm{~s}$ pulses of NO gas. Inset: Scheme of the device. Adapted from Ref. ${ }^{185}$ with permission from American Chemical Society. (c) TEM image of Ag-S-rGO and photographs of the sensing device printed on PI. (d) Response of the Ag-S-rGO sensor as a function of time in various concentrations of $\mathrm{NO}_{2}$ gas. Adapted from Ref. ${ }^{242}$ with permission from American Chemical Society.

Hybrid materials composed by graphene and metal oxides nanostructures ${ }^{184,243-249}$ or quantum dots 250,251 have also proved to enhance the sensing performances of graphene to $\mathrm{NO}_{2}$, ethanol, $\mathrm{H}_{2} \mathrm{~S}$ and other gases. In fact, semiconducting metal oxides such as $\mathrm{ZnO}, \mathrm{SnO}_{2}$, $\mathrm{In}_{2} \mathrm{O}_{3}$, and $\mathrm{Cu}_{2} \mathrm{O}$, have been already employed in gas sensing, ${ }^{252,}{ }^{253}$ yet the high operating temperature and low conductivity represented a severe limit towards their application. Deng et al. 243 synthetized a hybrid $\mathrm{rGO} / \mathrm{Cu}_{2} \mathrm{O}$ nanowire mesocrystals under hydrothermal conditions and integrated it in a $\mathrm{NO}_{2}$ gas sensor. The hybrid sensor displayed a higher sensitivity compared to that of the single components alone, with an impressive detection limit of $64 \mathrm{ppb}$ (Figure $8 \mathrm{a}-\mathrm{b})$. Also in this case the response resulted from the electron withdrawing effect of $\mathrm{NO}_{2}$ towards the $p$-type $\mathrm{rGO}$ and $\mathrm{Cu}_{2} \mathrm{O}$ semiconducting mesocrystals, and it took further advantage of the higher porosity of the hybrid material. An ethanol sensor was assembled by Yi et al. ${ }^{244}$ growing semiconductive vertically aligned $\mathrm{ZnO}$ nanorods on a metal bottom electrode and deposing CVD few-layers graphene on top as top contact. This sensor, with 
geometry similar to metal-insulator-metal devices, offered a $900 \%$ sensitivity to $10 \mathrm{ppm}$ of ethanol and a good flexibility. However, an operating temperature as high as $300{ }^{\circ} \mathrm{C}$ was required to leverage the $n$-type characteristic of semiconductive $\mathrm{ZnO}$ nanorods and obtain a good sensitivity to the electron donor EtOH. In another work ${ }^{245} \mathrm{GO}$ decorated with ZnO NPs was used to sense $\mathrm{CO}$ and $\mathrm{NH}_{3}$ gases. $\mathrm{ZnO}$ NPs were grown in solution on the GO surface; such a decorated GO was spin-coated on ITO patterned glass substrates. This device, when operated at room temperature, revealed a much higher sensitivity towards $\mathrm{CO}$ and $\mathrm{NH}_{3}$ compared with the two components alone. In particular, the change in conductivity was ca. $24 \%$ to $1 \mathrm{ppm}$ of $\mathrm{NH}_{3}$ and $22 \mathrm{ppm}$ of CO. Similarly, Cuong et al. ${ }^{246}$ reported a $\mathrm{H}_{2} \mathrm{~S}$ sensor based on vertically aligned $\mathrm{ZnO}$ nanorods hydrothermally grown on the surface of spraycoated rGO which showed high sensitivity in oxygen, but long response and recovery times. Another $\mathrm{H}_{2} \mathrm{~S}$ sensor was developed by Zhou et al. ${ }^{247}$ by hydrothermally growing $\mathrm{Cu}_{2} \mathrm{O}$ nanocrystals onto the GO surface and drop-casting the suspension onto a gold IDE on $\mathrm{Si} / \mathrm{SiO}_{2}$ substrate. The $\mathrm{GO} / \mathrm{Cu}_{2} \mathrm{O}$ assembly was demonstrated to have better performances compared to the isolated components, with a high $11 \%$ sensitivity to $5 \mathrm{ppb}$ of $\mathrm{H}_{2} \mathrm{~S}$ at room temperature and responses and recovery times on the few minutes time scale. Assembly of $\mathrm{rGO} / \mathrm{In}_{2} \mathrm{O}_{3}$ have been used to detect $\mathrm{NO}_{2}{ }^{249,254}$ for environmental monitoring. Feng et al. ${ }^{254}$ were able to embed $\mathrm{In}_{2} \mathrm{O}_{3}$ nanocubes in rGO networks using InN NWs and GO as precursors. Depending on the ratio between the two components, different morphologies were obtained: the best sensing performances were found when a 1:1 InN:GO ratio was used (Figure 8c). Such a sensor exhibited a $61 \%$ change in the resistance when exposed to $5 \mathrm{ppm}$ of $\mathrm{NO}_{2}$ and a markedly high selectivity towards interfering gases (Figure 8d). In 2017 Liu et al. ${ }^{249}$ overtook these performances by combining flower-shaped $\mathrm{In}_{2} \mathrm{O}_{3}$ nanocrystals with rGO thereby obtaining a sensor with an impressive 3 orders of magnitude increase in the resistance when exposed to $1 \mathrm{ppm}$ of $\mathrm{NO}_{2}$ combined with a detection limit lower than $10 \mathrm{ppb}$.

The sensing of simple alkanes, as methane and ethane is important because of the industrial relevance of these molecules. However, very few examples of sensing of these gases can be found in literature because of the weak doping nature of these apolar molecules. ${ }^{255-257}$ For example a hydrogen and liquid petroleum gas (LPG) sensor based on rGO decorated with $\mathrm{SnO}_{2}$ quantum dots has been developed by Lee et al. ${ }^{251}$, showing an impressive response of $89 \%$ to $500 \mathrm{ppm}$ of $\mathrm{H}_{2}$ and $92 \%$ to $500 \mathrm{ppm}$ of LPG. Zhang et al. ${ }^{255}$ produced a methane sensor based on a nanocomposite of rGO and $\mathrm{ZnO}$ nanocrystals that displayed a decent and fast response to $100 \mathrm{ppm}$ of $\mathrm{CH}_{4}$ and a good selectivity towards interfering gases; 
unfortunately a high operating temperature of $190{ }^{\circ} \mathrm{C}$ is necessary to properly function this sensor.

(a)

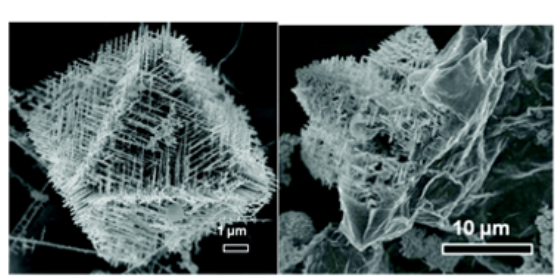

(b)

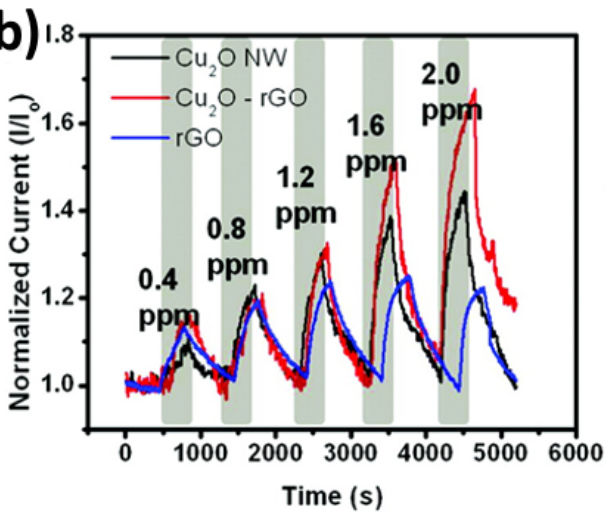

(c)
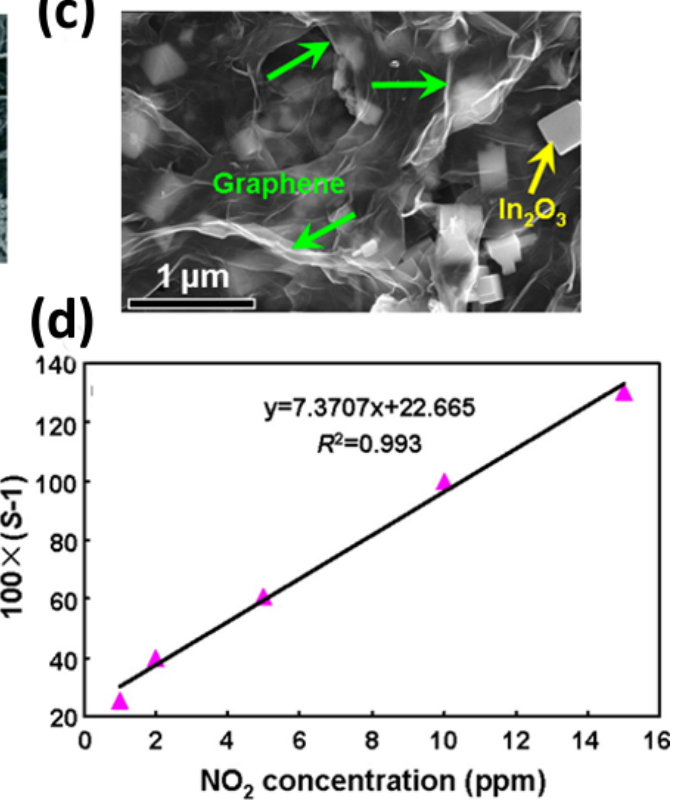

Figure 8. (a) SEM images of $\mathrm{rGO}-\mathrm{Cu}_{2} \mathrm{O}$ mesocystal composites, and (b) dynamic response of $\mathrm{Cu}_{2} \mathrm{O}$ NW, rGO-Cu $\mathrm{Cu}_{2} \mathrm{O}$, and $\mathrm{rGO}$ devices under increasing $\mathrm{NO}_{2}$ exposure. Adapted from Ref. 243 with permission from American Chemical Society. (c) SEM images of the $\operatorname{In}_{2} \mathrm{O}_{3}$ cubes/rGO composites at 1:1 mass ratio. (d) Linear fitting curve of the sensor response versus $\mathrm{NO}_{2}$ concentration. Adapted from Ref. ${ }^{254}$ with permission from American Chemical Society.

Polymers and semiconducting polymers coupled with graphene, GO and rGO constitute the third large family of hybrid assemblies. Some of these assemblies with conducting polymers, like polyaniline/GO assembly, have already been discussed in the previous paragraph ${ }^{232}$ as the boundary between polymer functionalized $\mathrm{GO} / \mathrm{rGO}$ and polymer- $\mathrm{GO} / \mathrm{rGO}$ assemblies is blurred. In a typical example, in 2014 Zhang et al. ${ }^{221}$ developed a resistive-type, flexible humidity sensor based on the assembly of GO and a polyelectrolyte, i.e. poly(diallylimethyammonium chloride) (PDDA), by using layer-by-layer (LbL) deposition method. The nanostructured film was fabricated on a $\mathrm{Cu} / \mathrm{Ni}$ IDE on PI substrate. In first instance a bi-layer of PDDA and an ionic polymer was self-assembled on the substrate as precursor layers for charge enhancement, and then 5 GO/PDDA layers were deposited by alternating immersions into PDDA and GO suspensions for five repetitive cycles. Following, the GO was chemically reduced by soaking the film in solution of $\mathrm{HBr}$ acid. Such an assembled device exhibited a $37 \%$ increase in the resistance when passing from $0 \% \mathrm{RH}$ to 
$97 \% \mathrm{RH}$, with a response and recovery time of a few minutes, an excellent linearity of the response and a long stability over time. The superior sensor performances were attributed to high surface area of the assembly, the $p$-type semiconducting properties of $\mathrm{rGO}$ at low $\mathrm{RH}$, and to the interlayer swelling of PDDA/RGO film at high $\mathrm{RH}$, which contributes to the resistance increase. In another work Shi et al. ${ }^{258}$ synthetized GO/conducting polymer composite hydrogels, including GO/polypirrole (PPy) and GO/PANI, by chemical polymerization in situ of the corresponding monomers in aqueous dispersions of GO, where GO sheets acted as 2D template. These gels showed many interesting properties, including electrical conductivity and electrochemical activity. In particular, the GO/PPy gel, once lyophilized, exhibited a good sensitivity to $\mathrm{NH}_{3}$ gas. In this case, the improved response was attributed to the high surface area of the conductive gel compared to the bare polymer film.

A nanocomposite thin film of chemically exfoliated graphene and PANI was used by Wu et $a l .{ }^{257}$ to sense methane at room temperature, with a decent $10 \%$ response to $10 \mathrm{ppm}$ of gas. Shi et al. ${ }^{259}$ prepared a highly sensitive $\mathrm{NO}_{2}$ sensor based on electrospun PVA/PEI nanofibers coated with a thin GO layer, which self-assembled on the surface of the nanofibers for charge interaction. The nanofibers were deposited on an IDE electrode and then exposed to hydrazine vapours to reduce the GO. This sensor displayed an impressive sensitivity, with a $16 \%$ response to $150 \mathrm{ppb}$ of $\mathrm{NO}_{2}$ and good linearity until $1 \mathrm{ppm}$; furthermore, the stability and the selectivity towards $\mathrm{NO}_{2}$ were high, and the response and recovery time were around 5 minutes.

The combination of graphene with ionic liquid is a similar route to achieve high sensitivity and selectivity in gas sensing. Within this framework, Ariga et al. ${ }^{260}$ developed a sensor capable of discriminating between various organic vapours based on a multi-layered $\mathrm{rGO} /$ ionic liquid film assembled in solution via $\mathrm{LbL}$ method on a quartz microbalance. Assemblies of CNTs and graphene have been also employed for gas sensing. In 2010 Jeong et al. ${ }^{261}$ produced a flexible $\mathrm{NO}_{2}$ sensor using a CNTs/rGO hybrid film. A rGO film was deposited on a plastic substrate with a gold IDE followed by the CVD deposition of vertically aligned CNTs on top. The so-fabricated sensor revealed a sensitivity of $20 \%$ after $60 \mathrm{~min}$ exposure to $10 \mathrm{ppm} \mathrm{NO}_{2}$ at room temperature.

The most importing performance parameters of all the graphene based gas sensors described in this section, including the response time and the sensitivity (calculated as the ratio between 
the response $\%$ and the corresponding gas concentration expressed in ppm) are summarized in Table 1. 
Table 1. Comparison of the sensing performances of different graphene based gas sensors.

\begin{tabular}{|c|c|c|c|c|c|}
\hline Material & Sensed gases & $\begin{array}{c}\text { Response/recovery } \\
\text { time }\end{array}$ & $\begin{array}{l}\text { Limit of } \\
\text { detection }\end{array}$ & $\begin{array}{l}\text { Sensitivity } \\
\text { (Response } \\
\left.\quad{ }^{*} p_{p m^{-1}}\right)\end{array}$ & Ref. \\
\hline \multirow[t]{4}{*}{$S L G(M E)$} & $\mathrm{NO}_{2}$ & $500 \mathrm{~s}$ & ppb & $4 \%$ & 201 \\
\hline & $\begin{array}{c}\mathrm{H}_{2} \mathrm{O} \text {, nonanal, octanoic } \\
\text { acid }\end{array}$ & & $0.5 \mathrm{ppm}$ & $0.6-1 \%$ & 204 \\
\hline & $\mathrm{CO}_{2}$ & $10 \mathrm{~min} / 10 \mathrm{~min}$ & & $0.26 \%$ & 206 \\
\hline & $\mathrm{NO}_{2}$ & $4 \mathrm{~min} / 4 \mathrm{~min}$ & 100 ppm & $0.08 \%$ & 80 \\
\hline$S L G / B L G(M E)$ & $\begin{array}{c}\text { THF, } \mathrm{MeOH}, \mathrm{MeCN}, \\
\mathrm{CHCl}_{3}\end{array}$ & & & & 205 \\
\hline$S L G(C V D)$ & $\mathrm{CH}_{4}, \mathrm{NH}_{3}, \mathrm{H}_{2}$ & $9 \mathrm{~min} / 9 \mathrm{~min}$ & & $\begin{array}{c}0.046 \% \\
\left(\mathrm{NH}_{3}\right)\end{array}$ & 211 \\
\hline$S L G / B L G(C V D)$ & $\mathrm{NO}_{2}$ & $95 \mathrm{~s} / 11 \mathrm{~s}$ & & $0.98 \%$ & 209 \\
\hline $\begin{array}{l}S L G(C V D) \text { oxidized with } \\
\qquad \mathrm{O}_{3}\end{array}$ & $\mathrm{NO}_{2}$ & $15 \mathrm{~min} / 30 \mathrm{~min}$ & & $0.0085 \%$ & 208 \\
\hline$S L G(C V D)$ patterned & $\mathrm{NO}_{2}, \mathrm{NH}_{3}$ & $89 \mathrm{~s} / 579 \mathrm{~s}$ & & $\begin{array}{l}2.6 \% \\
\left(\mathrm{NO}_{2}\right)\end{array}$ & 210 \\
\hline CVD graphene Foam & $\mathrm{NH}_{3}$ & $800 \mathrm{~s} / 1200 \mathrm{~s}$ & & $0.03 \%$ & 212 \\
\hline \multirow[t]{2}{*}{ SLG (epitaxially grown) } & $\mathrm{NO}_{2}$ & $1 \mathrm{~h} / 3 \mathrm{~h}$ & & $48 \%$ & 216 \\
\hline & $\mathrm{NO}_{2}$ & $\begin{array}{c}250 \mathrm{~s} / 150 \mathrm{~s} \text { at } 300 \\
{ }^{\circ} \mathrm{C}\end{array}$ & & $0.55 \%$ & 214 \\
\hline \multirow[t]{4}{*}{$G O$} & $\mathrm{H}_{2} \mathrm{O}$ & $20 \mathrm{~ms} / 20 \mathrm{~ms}$ & & $30 \% / \mathrm{RH}$ & 227 \\
\hline & $\mathrm{NO}_{2}, \mathrm{H}_{2} \mathrm{O}$ & $40 \mathrm{~min} / 40 \mathrm{~min}$ & & $12 \%$ & 219 \\
\hline & $\mathrm{NO}_{2}$ & $40 \mathrm{~min} / 40 \mathrm{~min}$ & $20 \mathrm{ppb}$ & $250 \%$ & 226 \\
\hline & $\mathrm{H}_{2} \mathrm{O}$ & & & $12.5 \%$ & 230 \\
\hline \multirow[t]{5}{*}{$r G O$} & $\begin{array}{c}\text { Acetone, DMMP, } \\
\text { HCN }\end{array}$ & $5 \mathrm{~s}$ & & $100 \%$ & 73 \\
\hline & $\mathrm{NO}_{2}, \mathrm{Cl}_{2}, \mathrm{NH}_{3}$ & $10 \mathrm{~min} / 10 \mathrm{~min}$ & & $\begin{array}{l}0.25 \% \\
\left(\mathrm{NH}_{3}\right)\end{array}$ & 224 \\
\hline & $\mathrm{H}_{2} \mathrm{O}$ & & & & 231 \\
\hline & $\mathrm{NO}_{2}$ & $10 \mathrm{~min} / 30 \mathrm{~min}$ & & $0.05 \%$ & 225 \\
\hline & $\mathrm{H}_{2} \mathrm{O}$ & $20 \mathrm{~s} / 30 \mathrm{~s}$ & & $10.7 \% / \mathrm{RH}$ & 148 \\
\hline \multirow{3}{*}{$\begin{array}{c}\text { Li and } B \text { doped } G O \\
\text { Sulfonated-GO and EDA- } \\
\text { GO } \\
\text { phenyl-GO, dodecyl-GO; } \\
\text { ethanol-GO }\end{array}$} & $\mathrm{H}_{2} \mathrm{O}$ & $4 \mathrm{~s}$ & & $35.3 \%$ & 228 \\
\hline & $\mathrm{NO}_{2}$ & & & $50 \%$ & 234 \\
\hline & Various gases & $15 \mathrm{~s} / 10 \mathrm{~s}$ & & $\begin{array}{l}200 \% \\
\left(\mathrm{NH}_{3}\right)\end{array}$ & 200 \\
\hline p-phenylenediamine/GO & DMMP & $1080 \mathrm{~s} / 360 \mathrm{~s}$ & & $0.4 \%$ & 223 \\
\hline
\end{tabular}




\begin{tabular}{|c|c|c|c|c|c|}
\hline$P A N I / r G O$ & $\mathrm{H}_{2}$ & $2 \mathrm{~min} / 3 \mathrm{~min}$ & & $0.0016 \%$ & 232 \\
\hline PANI/Graphene flakes & $\mathrm{CH}_{4}$ & $1 \mathrm{~min} / 1 \mathrm{~min}$ & $10 \mathrm{ppm}$ & $2.5 \%$ & 257 \\
\hline PDDA/GO LbL assembly & $\mathrm{H}_{2} \mathrm{O}$ & $2 \mathrm{~min} / 3 \mathrm{~min}$ & & $0.38 \% / \mathrm{RH}$ & 221 \\
\hline microfiber PVA-PEI/rGO & $\mathrm{NO}_{2}$ & $4 \mathrm{~min} / 10 \mathrm{~min}$ & & $110 \%$ & 259 \\
\hline$C N T s / G O$ & $\mathrm{NO}_{2}$ & $1 \mathrm{~h} / 3 \mathrm{~h}$ & $0.5 \mathrm{ppm}$ & $2 \%$ & 261 \\
\hline Ag NPs/Sulfonated-GO & $\mathrm{NO}_{2}, \mathrm{NH}_{3}$ & $12 \mathrm{~s} / 20 \mathrm{~s}$ & & $\begin{array}{l}1.49 \% \\
\left(\mathrm{NO}_{2}\right)\end{array}$ & 242 \\
\hline$P d$ film/CVD SLG & $\mathrm{H}_{2}$ & $>10 \min$ & & $0.0012 \%$ & 237 \\
\hline$P d N P S / C V D S L G$ & $\mathrm{H}_{2}$ & $15 \mathrm{~min} / 20 \mathrm{~min}$ & & $0.003 \%$ & 238 \\
\hline$P d / r G O$ & NO & $265 \mathrm{~s}$ & & $35 \%$ & 185 \\
\hline Pt $N P s / G O$ & $\mathrm{H}_{2}$ & $9 \mathrm{~min} / 20 \mathrm{~min}$ & & $0.004 \%$ & 235 \\
\hline $\mathrm{Cu}_{2} \mathrm{O} \mathrm{NPS} / \mathrm{rGO}$ & $\mathrm{H}_{2} \mathrm{~S}$ & $5 \mathrm{~min} / 5 \mathrm{~min}$ & & $2200 \%$ & 247 \\
\hline $\mathrm{Cu}_{2} \mathrm{O} / \mathrm{rGO}$ & $\mathrm{N}_{2} \mathrm{O}$ & $5 \mathrm{~min} / 10 \mathrm{~min}$ & & $34 \%$ & 243 \\
\hline $\mathrm{In}_{2} \mathrm{O}_{3} / r G \mathrm{O}$ & $\mathrm{NO}_{2}$ & $4 \mathrm{~min} / 1 \mathrm{~min}$ & & $109800 \%$ & 249 \\
\hline $\mathrm{In}_{2} \mathrm{O}_{3} / r G \mathrm{O}$ & $\mathrm{NO}_{2}$ & $3 \mathrm{~min} / 4 \mathrm{~min}$ & & $12.2 \%$ & 254 \\
\hline $\mathrm{ZnO} N \mathrm{NP} / \mathrm{rGO}$ & $\mathrm{CO}, \mathrm{NH}_{3}$ & $5 \mathrm{~min}$ & & $\begin{array}{l}24 \% \\
\left(\mathrm{NH}_{3}\right)\end{array}$ & 245 \\
\hline $\mathrm{ZnO} \mathrm{Nrods} / \mathrm{rGO}$ & $\mathrm{H}_{2} \mathrm{~S}$ & $>30 \mathrm{~min}$ & & & 246 \\
\hline $\mathrm{ZnO}$ Nrods/CVD SLG & $\mathrm{EtOH}$ & & & $90 \%$ & 244 \\
\hline ZnO NCryst/rGO & $\mathrm{CH}_{4}$ & $1 \mathrm{~min} / 10 \mathrm{~s}$ & 100 ppm & $0.05 \%$ & 255 \\
\hline
\end{tabular}

List of abbreviations. SLG : single layer graphene, ME : mechanically exfoliated, BLG : Bi-layer graphene, CVD : chemical vapour deposition, $\mathrm{GO}$ : graphene oxide, $\mathrm{rGO}$ : reduced graphene oxide, $\mathrm{CHCl}_{3}$ : chloroform, THF : tetrahydrofuran, $\mathrm{MeOH}$ : methanol, MeCN: acetonitrile, DMMP : dimethyl methylphosphonate, HCN : hydrogen cyanide, EDA : ethylenediamine, PANI : polyaniline, PDDA : poly(diallylimethyammonium chloride, LbL : layer-by-layer, PVA : polyvinyl alcohol, PEI : polyethylenimine, CNTs : carbon nanotubes, NPs : nanoparticles, Nrods : nanorods, NCryst: nanocrystals.

\subsection{Gas sensing with transition metal dichalcogenides}

The sensing properties of TMDs are based on the already discussed charge-transfer mechanism. Yue et al. ${ }^{191}$ attained a deep insight into such a mechanism by focusing their attention to the $n$-type $\mathrm{MoS}_{2}$ as exemplary system. In this article, the charge transfer mechanism between different gas molecules including $\mathrm{O}_{2}, \mathrm{H}_{2} \mathrm{O}, \mathrm{NH}_{3}, \mathrm{NO}, \mathrm{NO}_{2}, \mathrm{CO}$, etc. and monolayer $\mathrm{MoS}_{2}$ was explained with the aid of DFT calculations. The authors showed that the conduction band (CB) of pristine $n$-type $\mathrm{MoS}_{2}$ monolayers is already populated by some electrons at room temperature. When the monolayer is exposed to electron-acceptor gases such as $\mathrm{O}_{2}, \mathrm{H}_{2} \mathrm{O}, \mathrm{NO}, \mathrm{NO}_{2}$, and $\mathrm{CO}$, electrons are transferred from $\mathrm{MoS}_{2}$ to the sensitive gases, leading to a decrease of carrier density in $\mathrm{MoS}_{2}$ which ultimately yields an increased 
resistance. On the contrary $\mathrm{NH}_{3}$, which behaves as an electron-donor, transfers electrons to the $\mathrm{MoS}_{2}$ monolayer thereby reducing its resistance.

The charge transfer between TMDs monolayers and gases can also be evidenced by changes in the photoluminescence (PL), which is due to the direct band gap properties of TMDs monolayers. Tongay et al. ${ }^{262}$ showed that the light emission efficiency of these TMDs can be modulated by physisorption of gas molecules such as $\mathrm{O}_{2}$ and $\mathrm{H}_{2} \mathrm{O}$, as a result of a molecular gating effect. The charge depletion in $n$-type materials such as $\mathrm{MoS}_{2}$ and $\mathrm{MoSe}_{2}$ caused by the charge transfer to molecules such as $\mathrm{O}_{2}$ and $\mathrm{H}_{2} \mathrm{O}$ leads to the drastic enhancement in photoluminescence for the stabilization of neutral excitons $\mathrm{X}_{0}$.

Among the various TMDs, $\mathrm{MoS}_{2}$ is the most studied material. Mechanically exfoliated $\mathrm{MoS}_{2}$ has been successfully used to detect a wide range of gases. ${ }^{14,190,193,263}$ For instance Zhang et $a l .{ }^{190}$ fabricated miniaturized FETs based on mechanically exfoliated $\mathrm{MoS}_{2}$ for the detection of NO. Single, bi, tri and quadri-layers of $\mathrm{MoS}_{2}$ transferred on $\mathrm{Si} / \mathrm{SiO}_{2}$ substrates were investigated in this work. The sensitivity of these devices to NO was high and the response fast; however, the current passing through a single-layer thick $\mathrm{MoS}_{2}$ FET was unstable while the devices based on 2, 3 and 4-layers $\mathrm{MoS}_{2}$ showed both high sensitivity and stability with a detection limit of $0.8 \mathrm{ppm}$ of NO. In a similar work Late et al. ${ }^{14}$ prepared transistor deposing mechanically exfoliated $\mathrm{MoS}_{2}$ flakes on $\mathrm{Si} / \mathrm{SiO}_{2}$ substrates and contacting them with gold top electrodes through electron beam lithography and they employed them to sense $\mathrm{NO}_{2}, \mathrm{NH}_{3}$ and humidity (Figure 9a). From the analysis of flakes consisting of 1,2 and 5-layers it was observed that the signal was unstable for the case of monolayers while among 2 and 5-layers the latter exhibited the highest response to the sensed gases. As expected from the charge transfer mechanism $\mathrm{NO}_{2}$ produced a negative resistance response while $\mathrm{NH}_{3}$ had the opposite behaviour (Figure $9 \mathrm{~b}$ and $8 \mathrm{c}$ ). For the same reason the applied gate voltage had opposite effects in $\mathrm{NH}_{3}$ and $\mathrm{NO}_{2}$ in increasing or decreasing the sensitivity and these effects were larger for the 5-layers device. In addition, the effects of green light irradiation over the sensitivity, and response time of the device was also explored. The reason for the increased and stable response in the devices based on few-layers compared to the single-layer thick remains unclear, yet the authors showed with DFT calculations that the adsorption of $\mathrm{NO}_{2}$ was slightly favoured in the few-layers thick devices. In another example Perkins et al. ${ }^{193}$ measured the conductance changes in a device based on mechanically exfoliated $\mathrm{MoS}_{2}$ after exposing it to various organic vapours. They found out that the device had high selectivity 
towards triethylamine (TEA) and acetone with a detection limit of $1 \mathrm{ppm}$ over the last analyte.
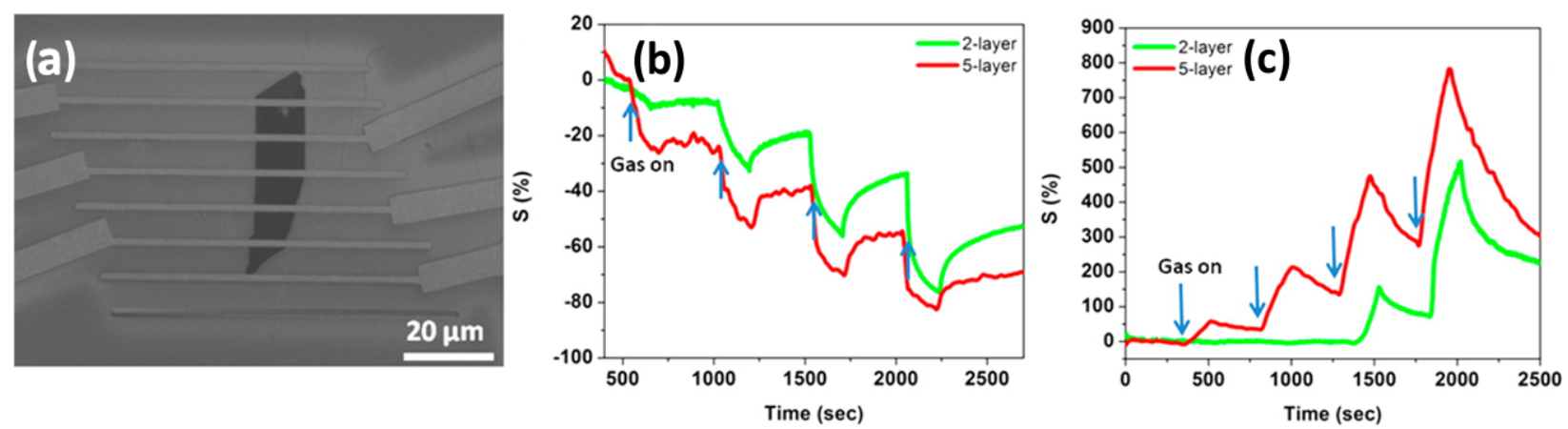

Figure 9. (a) SEM image of two-layer $\mathrm{MoS}_{2}$ transistor device. Sensing performances of the 2 and 5-layers devices when exposed to 100, 200, 500, 1000 ppm of (b) $\mathrm{NH}_{3}$, and (c) $\mathrm{NO}_{2}$. Adapted from Ref. ${ }^{14}$ with permission from the American Chemical Society.

Dispersion of $\mathrm{MoS}_{2}$ sheets in solution can be obtained with a relatively high yield and low costs by liquid-phase exfoliation. These dispersions can be processed to generate films of overlapping flakes, which have been used as active materials to detect different gases. ${ }^{192,264,}$ ${ }^{265}$ For example Yao et al. ${ }^{265}$ prepared a water dispersion of $\mathrm{MoS}_{2}$ flakes combining grinding and sonication of the bulk material. The dispersion was deposited with an inject printer onto pre-patterned microelectrodes and the so-obtained device was used to detect $\mathrm{NH}_{3}$ gas up to a few ppm. A similar method that relies on the intercalation of Li ions within the $\mathrm{MoS}_{2}$ layers and the subsequent exfoliation of these in water solution through ultrasonication has been used by Zhang et al. ${ }^{141}$ to sense NO. The dispersion of $\mathrm{MoS}_{2}$ flakes was deposited by dropcasting on a $\mathrm{Si} / \mathrm{SiO}_{2}$ substrate pre-patterned with gold electrodes to form a thin-film transistor (TFT) which was found being sensitive to NO in concentrations down to $0.4 \mathrm{ppm}$.

Similarly to graphene, also $\mathrm{MoS}_{2}$ can be produced by CVD. ${ }^{143}$ In 2014 Liu et al. ${ }^{144}$ fabricated FETs by contacting through EBL small triangular-shaped monolayers of $\mathrm{MoS}_{2}$ grown by $\mathrm{CVD}$ on $\mathrm{Si} / \mathrm{SiO}_{2}$ substrates (

Figure 10a). These devices exhibited a high Schottky barrier $(\Delta \mathrm{SB})$ between the $\mathrm{Ti} / \mathrm{Au}$ contacts and the monolayer, which can be modulated by exposing the devices to $\mathrm{NO}_{2}$ and $\mathrm{NH}_{3}$ gases. In particular, $\mathrm{NO}_{2}$ adsorption increases the $\Delta \mathrm{SB}$ and thus of the contact resistance, whereas the $\mathrm{NH}_{3}$ adsorption determines a decrease of both them. The effect of the Schottky 
barrier modulation, together with the charge transfer mechanism, yielded an increase in the sensitivity of the device to both the analytes, with changes in the conductance up to 3 orders of magnitude and detection limits down to $20 \mathrm{ppb}$ of $\mathrm{NO}_{2}$ and $1 \mathrm{ppm}$ of $\mathrm{NH}_{3}$ (

Figure 10b). Cho et al. ${ }^{146}$ produced a gas and photo-detector based on wafer-scale film of 3layers $\mathrm{MoS}_{2}$ deposited on sapphire substrates via CVD with IDE gold electrodes on top. This sensor exhibited good sensitivity and selectivity to $\mathrm{NO}_{2}$, with a response around $40 \%$ to 120 ppb combined with a stable cycling behaviour. Furthermore, the response time was fast, yet the recovery at room temperature was not complete even after 30 minutes. Similarly, a 3layers $\mathrm{MoS}_{2}$ film produced by CVD on sapphire substrates was developed by Cho et al. ${ }^{266}$ for the sensing of $\mathrm{NO}_{2}$ and $\mathrm{NH}_{3}$. The device exhibited an increased resistance when exposed to $\mathrm{NO}_{2}$ and a decreased resistance when interacting with $\mathrm{NH}_{3}$, as expected from the chargetransfer mechanism.

The sensitivity of $\mathrm{MoS}_{2}$ to gases could be improved by increasing the number of active sites that can interact with the analyte of interest through processes of molecular absorption on sulphur vacancies and edges. For example Jung et al. ${ }^{147}$ showed that sensors based on $\mathrm{MoS}_{2}$ layers vertically aligned, thus exposing their edges to the environment, exhibited a higher response to $\mathrm{NO}_{2}$ and EtOH gases. The films of horizontally and vertically aligned $\mathrm{MoS}_{2}$ layers were produced via CVD. By increasing the thickness of the films, the number of vertically aligned layers increased, and at a thickness of $15 \mathrm{~nm}$ almost all the surface was covered by vertically aligned layers, as revealed by TEM mapping. These films exhibited a response 5 times greater than the $1 \mathrm{~nm}$ thick film, because of the increased edge sites exposed to the environment.

The surface functionalization of $\mathrm{MoS}_{2}$ with molecules or nanomaterials as metal and metal oxides NPs represents an alternative viable method to increase the interactions with the gas molecules, thus to harness the device sensitivity. ${ }^{267-268}$ For example Zhang et al. ${ }^{267}$ fabricated a flexible TFT deposing a film of $\mathrm{MoS}_{2}$ obtained with the method previously described ${ }^{141}$ on an rGO electrode array on polyethylene terephthalate (PET). Successively, Pt NPs were deposited on the surface of the $\mathrm{MoS}_{2}$ film and the device was employed to detect $\mathrm{NO}_{2}$. The device functionalized with the Pt NPs showed a response to $\mathrm{NO}_{2}$ being 3 times faster than the not-functionalized device, with detection limits as low as $2 \mathrm{ppb}$. The reason for such an improved sensitivity was attributed to the formation of a Schottky barrier between the $\mathrm{MoS}_{2}$ and the Pt NPs. In a similar work Sarkar et co-workers ${ }^{269}$ functionalized the $\mathrm{MoS}_{2}$ 
with various metallic NPs, included $\mathrm{Ag}, \mathrm{Pd}, \mathrm{Pt}, \mathrm{Sc}$ and $\mathrm{Y}$ and examined their doping effect. The Pt NPs functionalized $\mathrm{MoS}_{2}$ device revealed an improved sensitivity to $\mathrm{H}_{2}$ gas compared to the pristine $\mathrm{MoS}_{2}$ device. An $\mathrm{H}_{2}$ sensor based on $\mathrm{Pd}$ NPs functionalized $\mathrm{MoS}_{2}$ was assembled also by Jin et al. ${ }^{268}$ by drop-casting of a water suspension of $\mathrm{MoS}_{2}$ flakes obtained by liquid-phase exfoliation and Pd NPs. This sensor displayed a fast response and good recovery upon exposure to of $\mathrm{H}_{2}(0.5-5 \%)$ and a decent cross-sensitivity. Metal oxides NPs have also been used to improve the sensitivity; in particular, the semiconducting $\mathrm{SnO}_{2}$ has been employed to sense humidity, ${ }^{198} \mathrm{NO}_{2}{ }^{270}$ and EtOH. ${ }^{271}$ For example Chen et al. ${ }^{270}$ prepared a $\mathrm{NO}_{2}$ sensor based on $\mathrm{SnO}_{2}$ nanocrystals decorated $\mathrm{MoS}_{2}$ nanosheets. The material was prepared as depicted in

Figure 10c. A MoS 2 flakes suspension was produced by lithiation of $\mathrm{MoS}_{2}$ followed by liquid exfoliation, then $\mathrm{SnCl}$ was added at the suspension and $\mathrm{Sn}^{4+}$ ions got absorbed on the negatively charged flake's surface. Finally, the $\mathrm{SnO}_{2} / \mathrm{MoS}_{2}$ hybrid was obtained by annealing of the filtered suspension at $300{ }^{\circ} \mathrm{C}$ in argon. Interestingly, this material showed $p$-type behaviour because of the $p$-doping effect of the $\mathrm{SnO}_{2}$ nanocrystals. The sensor based on the hybrid showed a decent sensitivity to $\mathrm{NO}_{2}$, with a $30 \%$ response to $10 \mathrm{ppm}$ and detection limit of 0.5 ppm combined with a good reversibility and selectivity (

Figure 10d).
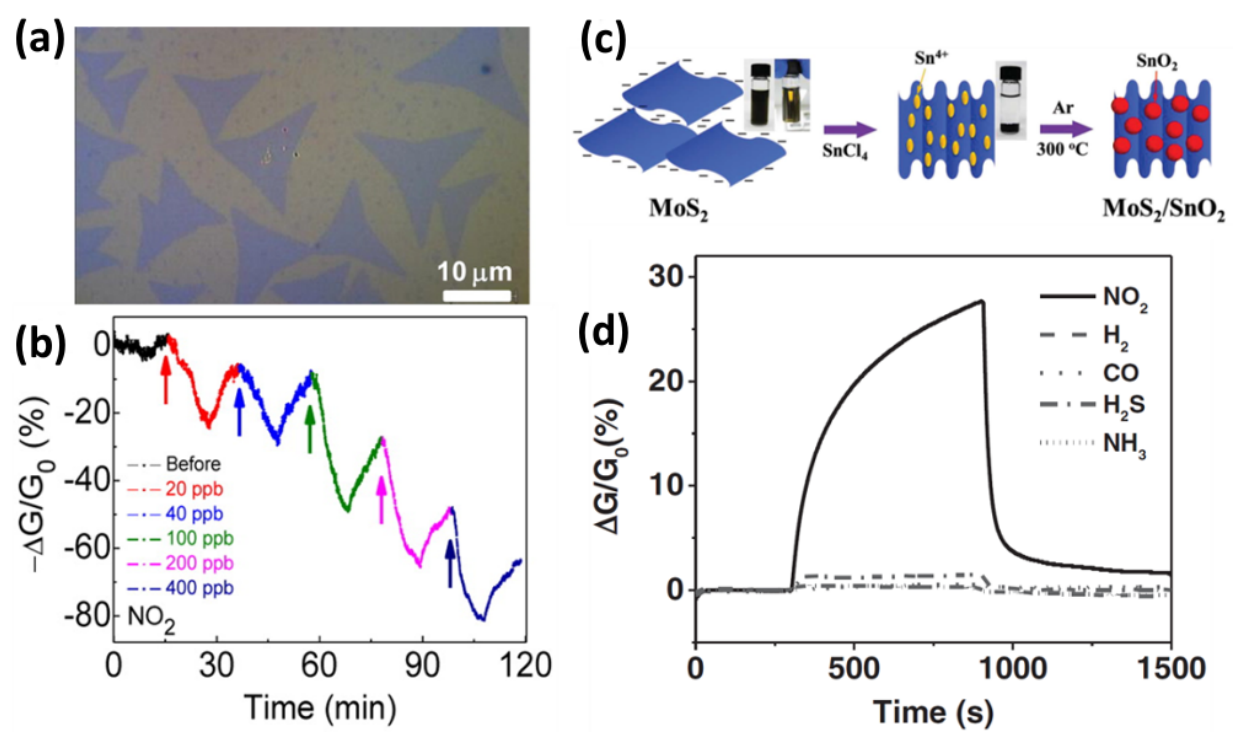

Figure 10. (a) Micrograph of the triangular shaped of CVD grown $\mathrm{MoS}_{2}$ monolayers supported on a $\mathrm{Si} / \mathrm{SiO}_{2}$ substrate. (b) Response of the device under the exposure to increasing $\mathrm{NO}_{2}$ concentrations. Adapted from Ref. ${ }^{144}$ with permission from American Chemical 
Society. (d) Schematic illustration of the preparation of the $\mathrm{SnO}_{2} / \mathrm{MoS}_{2}$ hybrid. (d) Response of the device to $10 \mathrm{ppm}$ of various gases, highlighting the selectivity towards $\mathrm{NO}_{2}$, the sensitivity and the response time. Adapted from Ref. ${ }^{270}$ with permission from Wiley-VHC.

$\mathrm{MoS}_{2}$ can also be functionalized with dangling molecules, especially through the covalent attachment of thiolated molecules on the sulphur vacancies of the $\mathrm{MoS}_{2}$ surface. ${ }^{272}$ By applying such a strategy Jung et al. ${ }^{13}$ developed a volatile organic compounds (VOCs) sensor for breath analysis based on $\mathrm{MoS}_{2}$ nanosheets obtained via liquid-exfoliation functionalized with mercaptoundecanoic acid (MUA). This sensor exhibited a good sensitivity down to 1 ppm to various VOCs included toluene, hexane, acetone, propanale and ethanol. Furthermore, while the resistance of pristine $\mathrm{MoS}_{2}$ based device increased upon exposure to all the sensed gases, the MUA-MoS $\mathrm{S}_{2}$ sensor once exposed to the molecules with oxygen functionalities displayed a decrease in the resistance. These differences originated from the diverse interaction of the oxygen containing molecules with the dangling MUA. In particular, the oxygen-bearing gas molecules can interact via hydrogen bonding with the exposed carboxyl group in MUA and then form an electron-rich region able to transfer the charge to the $\mathrm{MoS}_{2}$ sensing channel.

Among the other TMDs, $\mathrm{MoSe}_{2}, \mathrm{WS}_{2}, \mathrm{WSe}_{2}, \mathrm{ReS}_{2}$ and $\mathrm{PtSe}_{2}$ have also been employed for fabricating gas sensing devices by exploiting their diverse semiconducting characteristics and their unique high surface-to-volume ratio. For example $\mathrm{MoSe}_{2}$ has found application as $\mathrm{NH}_{3}$ sensor in a work of Late et al. ${ }^{273}$ The device was based on a single-layer $\mathrm{MoSe}_{2}$ flake obtained via mechanical exfoliation deposited on a $\mathrm{Si} / \mathrm{SiO}_{2}$ substrate and contacted with gold electrodes by EBL. The device showed a sensitivity to $\mathrm{NH}_{3}$ in concentration from 50 to 500 ppm and a response time of $2.5 \mathrm{~min}$. The doping effect of $\mathrm{NH}_{3}$ was confirmed also by the shift in the Raman spectrum after the absorption of $\mathrm{NH}_{3}$.

$\mathrm{WS}_{2}$ has been used in recent years as a sensor for $\mathrm{NH}_{3},{ }^{52,194}$ humidity ${ }^{195,274}$ and alcohols. ${ }^{52}$, ${ }^{195} \mathrm{Li}$ et al. ${ }^{52}$ developed a transistor based on a mechanically exfoliated multilayer $\mathrm{WS}_{2}$ flake and measured the current change and the photoresponse of the transistor when exposed to different gases included $\mathrm{NH}_{3}$, ethanol and $\mathrm{O}_{2}$. In particular, $\mathrm{NH}_{3}$ and ethanol behaved as electron-donor, yielding an increase in current and photo-responsivity in the $n$-type $\mathrm{WS}_{2}$ device, while $\mathrm{O}_{2}$ extracted electrons from the device producing a current decrease and a lower photo-response. The device showed the highest sensitivity toward $\mathrm{NH}_{3}$ and really fast response and recovery to all the sensed gases. Pumera et al. ${ }^{195}$ prepared a chemiresistive 
device deposing metallic $1 \mathrm{~T}-\mathrm{WS}_{2}$ flakes obtained through liquid exfoliation on a IDE. The selective vapour sensing capabilities of the device were studied with impedance spectroscopy. When exposed to methanol the device presented an impedance phase spectrum with a resonant frequencies maximum around $1 \mathrm{~Hz}$, while with water the maximum shifted to $1 \mathrm{kHz}$. Therefore, the device was able to discern methanol and water isolated and mixed together by simply selecting specific frequencies, even when high concentrations of interfering gases were present. Duesberg et al. ${ }^{194}$ reported a $\mathrm{NH}_{3}$ sensor based on CVD WS 2 thin film. The films with thickness ranging from 1 to $50 \mathrm{~nm}$ were grown on $\mathrm{Si} / \mathrm{SiO}_{2}$ substrates with gold IDE by using a $\mathrm{H}_{2} \mathrm{~S}$ plasma to sulphurise $\mathrm{WO}_{3}$ films at $500{ }^{\circ} \mathrm{C}$. The so-achieved devices demonstrated good sensitivity to $\mathrm{NH}_{3}$, with a detection limit of $1.4 \mathrm{ppm}$.

$\mathrm{WSe}_{2}$ is another TMD semiconductor which has been used to detect toxic gases. Javey et al. 275 assembled a $p$-type FET based on mechanically exfoliated $\mathrm{WSe}_{2}$ monolayer for $\mathrm{NO}_{2}$ detection. A high work function metal as Pd was used as source and drain electrode to lower the contact resistance for holes injection. Upon exposure to $0.05 \%$ of $\mathrm{NO}_{2}$ the source-drain current increased of 5 magnitude orders as a result of the lowering of the Schottky barrier and increasing of $p$-doping. Furthermore, in devices with top-contact gate also the on-off ratio was greatly improved after $\mathrm{NO}_{2}$ exposure.

Recently also $\mathrm{ReS}_{2}, \mathrm{ReSe}_{2}$ and $\mathrm{PtSe}_{2}$ have been employed for gas sensing. ${ }^{276-279}$ Yan et al. reported the photoresponse of monolayer $\mathrm{ReS}_{2}{ }^{276}$ and $\mathrm{ReSe}_{2}{ }^{278}$ based FET under red light illumination in $\mathrm{NH}_{3}$ and $\mathrm{O}_{2}$ gases. The increased current was observed under illumination were higher in $\mathrm{NH}_{3}$ atmosphere for $\mathrm{ReS}_{2}$, while for $\mathrm{ReSe}_{2}$ were higher in $\mathrm{O}_{2}$ atmosphere. With the aid of DFT spin-polarized calculations the reason of the different photoresponse behaviour of single-layer $\mathrm{ReS}_{2}$ and $\mathrm{ReSe}_{2}$ FETs in $\mathrm{O}_{2}$ and $\mathrm{NH}_{3}$ was attributed to the different transfer direction and quantity of electrons in $n$-type $\mathrm{ReS}_{2}$ and $p$-type $\mathrm{ReSe}_{2}$. Yim et al. ${ }^{279}$ prepared a FET deposing a thin layer PtSe $e_{2}$ film by CVD on a $\mathrm{Si} / \mathrm{SiO}_{2}$ substrate and tested it as a photodetector and gas sensor. The FET exhibited a fast detection of $\mathrm{NO}_{2}$, with a resistance decrease of $0.25 \%$ to $1 \mathrm{ppm}$ of gas and response and recovery time of 30 and $10 \mathrm{~s}$ respectively.

\subsection{Gas sensing with other 2DMs}


$\mathrm{SnS}_{2}$ is a layered material similar to TMDs exhibiting a higher electronegativity, which can potentially enhance the gas sensing capabilities. Ou et al. ${ }^{280}$ developed a sensitive and selective $\mathrm{NO}_{2}$ sensor based on few-layer $\mathrm{SnS}_{2}$ flakes obtained by a wet chemical synthesis technique. The flakes, with lateral size ranging between 80 and $200 \mathrm{~nm}$, were synthetized from solution of $\mathrm{SnCl}_{4}$ and sulphur and then drop-casted on an IDE. The device displayed a linear response from 0.6 to $10 \mathrm{ppm}$ of $\mathrm{NO}_{2}$, with an extremely high sensitivity of $3500 \%$ to $10 \mathrm{ppm}$ of $\mathrm{NO}_{2}$ and response and recovery time of 170 and $140 \mathrm{~s}$, respectively, at an operating temperature of $120{ }^{\circ} \mathrm{C}$. Furthermore, the device showed high selectivity to $\mathrm{NO}_{2}$ among high concentration of various interfering gases; these results were attributed to the strong affinity of $\mathrm{SnS}_{2}$ for $\mathrm{NO}_{2}$ as well as the favourable Fermi level of $\mathrm{SnS}_{2}$ to the energy of the partially occupied $\mathrm{NO}_{2}$ molecular orbitals.

In another example, Shi et al. ${ }^{281}$ developed a $\mathrm{NH}_{3}$ sensor operating at room temperature based on flower-shaped nanoflakes of $\mathrm{SnS}_{2}$ prepared by a solution method. The sensing device was produced deposing a paste made of the interconnected flakes on a IDE. The sensor, when exposed to concentrations of $\mathrm{NH}_{3}$ ranging between 5 and $150 \mathrm{ppm}$, presented a $21 \%$ sensitivity to $5 \mathrm{ppm}$ of gas with response and recovery time of 40 and $100 \mathrm{~s}$, respectively. Another $\mathrm{NH}_{3}$ sensor based on $\mathrm{SnO}_{2} / \mathrm{SnS}_{2}$ hybrid flakes was fabricated more recently by Xu et $a l .{ }^{197}$ To form the hybrid structure, $\mathrm{SnS}_{2}$ flakes were firstly produced from a solution method and deposited on a gold IDE, then the device was heated to $300{ }^{\circ} \mathrm{C}$ in air in order to partially oxidase the surface to $\mathrm{SnO}_{2}$. This device showed good sensitivity to $\mathrm{NH}_{3}$, with a $16 \%$ response to $10 \mathrm{ppm}$ of $\mathrm{NH}_{3}$, which was much higher compared to the sample with not oxidized $\mathrm{SnS}_{2}$ or with the completely oxidized $\mathrm{SnO}_{2}$. The device exhibited also a good dynamic range from 10 to $500 \mathrm{ppm}$ of $\mathrm{NH}_{3}$, high reproducibility and a response time of just $11 \mathrm{~s}$.

Black phosphorus is a promising candidate as platform for gas sensing for its electrical properties and its sensitivity to the environments, which is usually considered as a drawback. ${ }^{153}$ First-principle calculations ${ }^{159}$ made it possible to cast light onto the interaction of many gases with phosphorene. The gas molecules can get physisorbed on the phosphorene surface and interact via van der Waals forces, yielding a modification of the electronic properties of phosphorene. $\mathrm{O}_{2}, \mathrm{NO}_{2}$ and $\mathrm{NO}$ act as strong electron acceptors whereas $\mathrm{CO}, \mathrm{H}_{2}, \mathrm{H}_{2} \mathrm{O}$ and $\mathrm{NH}_{3}$ act as electron donor. Abbas et al. firstly reported on the chemical sensing of $\mathrm{NO}_{2}$ using FET based on mechanically exfoliated multilayer phosphorene. ${ }^{158}$ The FET was produced by exfoliating the black phosphorus with the scotch-tape method on a $\mathrm{Si} / \mathrm{SiO}_{2}$ substrate and 
patterning gold electrodes by EBL. The sensor displayed increased conductivity upon $\mathrm{NO}_{2}$ exposure with a $3 \%$ response to $5 \mathrm{ppb}$ of the gas. Moreover, when the device was exposed to $\mathrm{NO}_{2}$ concentrations ranging from 5 to $40 \mathrm{ppb}$, its response complied with the Langmuir isotherm for molecules adsorbed on a surface, confirming the charge transfer as sensing mechanism. Cui et al. ${ }^{96}$ reported a similar FET based on phosphorene nanosheets exfoliated with the scotch-tape method for $\mathrm{NO}_{2}$ sensing. The sensor showed thickness dependent performance with sensitivities up to $190 \%$ to $20 \mathrm{ppb}$ of $\mathrm{NO}_{2}$, excellent stability and high selectivity in presence of interfering gases.

Phosphorene demonstrated good sensitivity also to organic vapors ${ }^{160}$ and humidity. ${ }^{282}$ Pumera et al. ${ }^{160}$ developed a highly selective methanol sensor based on black phosphorus plates deposited on a gold IDE. The device was studied with EIS: when exposed to methanol a strong signal at a resonance frequency of $1 \mathrm{kHz}$ appeared in the impedance phase spectrum and its intensity was proportional to the methanol concentration in the range of 380-1900 ppm. High reproducibility, long-term stability and excellent selectivity in presence of interfering gases were also established. Yasaei et $a .^{282}$ explored the possibility of employing of phosphorene films as humidity sensors. Phosphorene flakes were produced by ultrasonication in liquid and then filtered to obtain thick film of stacked flakes that were used as chemiresistors. When the humidity was increased from 10 to $85 \% \mathrm{RH}$ the conductivity increased by 4 orders of magnitude. According to EIS measurements it was hypothesized a sensing mechanism based on the formation of ionic charge carriers caused by ionization of the water molecules and solvation of the phosphorus oxoacids.

Hexagonal boron nitride possesses also promising properties, which render it an interesting material for gas sensing as it was assessed in few first-principles calculations. ${ }^{283}, 284$ However, the only example of application of $\mathrm{hBN}$ as gas sensor as been tendered by Feng et al. ${ }^{199}$ In particular, a $\mathrm{O}_{2}$ and $\mathrm{CH}_{4}$ sensor based on wafer-scale $\mathrm{hBN}$ nanosheets $(1.7 \mathrm{~nm}$ thick) by the $\mathrm{CO}_{2}$-pulsed laser deposition technique was developed. Such sensor revealed sensitivity to $100 \mathrm{ppm} \mathrm{O}_{2}$, of $150 \%$ with response and recovery time around $70 \mathrm{~s}$ and $100 \mathrm{~s}$, respectively. The sensitivity to $100 \mathrm{ppm} \mathrm{CH}_{4}$, was even greater, being around $780 \%$ with response and recovery times reduced to $15 \mathrm{~s}$ and $20 \mathrm{~s}$, respectively. The highest sensitivity towards $\mathrm{CH}_{4}$ could be caused by the higher polarizability compared to $\mathrm{O}_{2}$ which promotes its adsorption on the surface and at grain boundaries of $\mathrm{hBN}$, thereby changing its conductivity. 
The properties and the performances of the gas sensing devices based on TMDs and other layered 2DMs are summarized in Table 2. 
Table 2. Comparison of the sensing performances of different graphene based gas sensors.

\begin{tabular}{|c|c|c|c|c|c|}
\hline Material & Sensed gases & $\begin{array}{l}\text { Response/ } \\
\text { recovery time }\end{array}$ & $\begin{array}{l}\text { Limit of } \\
\text { detection }\end{array}$ & $\begin{array}{c}\text { Sensitivity } \\
\left(\text { Response }^{*} \mathrm{ppm}^{-1}\right)\end{array}$ & Ref. \\
\hline \multirow[t]{3}{*}{$M o S_{2}(M E)$} & NO & & $0.8 \mathrm{ppm}$ & & 190 \\
\hline & $\mathrm{NO}_{2}, \mathrm{NH}_{3}, \mathrm{H}_{2} \mathrm{O}$ & & & $1.372 \%\left(\mathrm{NO}_{2}\right)$ & 14 \\
\hline & TEA, acetone & $15 \mathrm{~s}, 30 \mathrm{~s}$ & $\begin{array}{l}1 \mathrm{ppm} \\
\text { TEA }\end{array}$ & $5 \%$ (TEA) & 193 \\
\hline \multirow[t]{2}{*}{$M o S_{2}(C V D)$} & $\mathrm{NO}_{2}, \mathrm{NH}_{3}$ & $>10 \mathrm{~min}$ & $5 \mathrm{ppm}$ & $2.5 \%\left(\mathrm{NO}_{2}\right)$ & 266 \\
\hline & $\mathrm{NO}_{2}$ & $1 \mathrm{~min}, 30 \mathrm{~min}$ & $120 \mathrm{ppb}$ & $333 \%$ & 146 \\
\hline \multirow{2}{*}{$\begin{array}{c}\mathrm{MoS}_{2}(C V D-S c h o t t k y \\
\text { contact }) \\
\mathrm{MoS}_{2}(C V D-\text { vertically } \\
\text { align. })\end{array}$} & $\mathrm{NO}_{2}, \mathrm{NH}_{3}$ & $10-20 \mathrm{~min}$ & $\begin{array}{c}20 \mathrm{ppb} \\
\mathrm{NO}_{2}, 1 \\
\mathrm{ppm} \mathrm{NH}_{3}\end{array}$ & $1000 \%\left(\mathrm{NO}_{2}\right)$ & 144 \\
\hline & $\mathrm{EtOH}, \mathrm{NO}_{2}$ & $10 \mathrm{~min}, 1 \mathrm{~h}$ & & $0.1 \%\left(\mathrm{NO}_{2}\right)$ & 147 \\
\hline \multirow{3}{*}{$\begin{array}{c}\mathrm{MoS}_{2}(L P E) \\
\mathrm{MoS}_{2}(L P E \text { in the presence } \\
\text { of mercaptoundecanoic } \\
\text { acid) } \\
\mathrm{MoS}_{2}-\text { array of devices } \\
\text { (LPE) }\end{array}$} & $\mathrm{NH}_{3}$ & & ppm & $5 \%$ & 265 \\
\hline & $\begin{array}{l}\text { VOCs (toluene, } \\
\text { EtOH, hexane, } \\
\text { acetone) }\end{array}$ & & & $0.003-0.0015 \%$ & 13 \\
\hline & $\mathrm{NO}_{2}$ & $1 \mathrm{~h}, 1 \mathrm{~h}$ & $2 \mathrm{ppb}$ & $10 \%$ & 267 \\
\hline$M o S_{2} /$ graphene electrodes & $\mathrm{NH}_{3}, \mathrm{NO}_{2}$ & & $\begin{array}{l}1.2 \mathrm{ppm} \\
\mathrm{NO}_{2}\end{array}$ & $\begin{array}{c}0.06 \%\left(\mathrm{NH}_{3}\right), 1.4 \\
\%\left(\mathrm{NO}_{2}\right)\end{array}$ & 263 \\
\hline \multirow[t]{2}{*}{$\mathrm{SnO}_{2} / \mathrm{MoS}_{2}$} & $\mathrm{NO}_{2}$ & $5 \mathrm{~min}, 1 \mathrm{~min}$ & $0.5 \mathrm{ppm}$ & $3 \%$ & 270 \\
\hline & $\mathrm{H}_{2} \mathrm{O}$ & & & $34883 \% / \mathrm{RH}$ & 198 \\
\hline $\mathrm{Pd} / \mathrm{MoS}_{2}$ & $\mathrm{H}_{2}$ & $40 \mathrm{~s}, 83 \mathrm{~s}$ & & $0.0032 \%$ & 268 \\
\hline \multirow[t]{2}{*}{$W S_{2}$} & $\mathrm{H}_{2} \mathrm{O}, \mathrm{MeOH}$ & & $\begin{array}{l}5.6 \mathrm{ppm} \\
\mathrm{MeOH}, \\
10 \% \mathrm{RH}\end{array}$ & & 195 \\
\hline & $\mathrm{NH}_{3}$ & ca. $10 \mathrm{~min}$ & $1.4 \mathrm{ppm}$ & $0.005 \%$ & 194 \\
\hline$W_{S e_{2}}$ & $\mathrm{NO}_{2}$ & & & $200 \%$ & 275 \\
\hline $\mathrm{PtSe}_{2}$ & $\mathrm{NO}_{2}$ & $30 \mathrm{~s}, 10 \mathrm{~s}$ & & $0.25 \%$ & 279 \\
\hline \multirow[t]{3}{*}{$\mathrm{SnS}_{2}$} & $\mathrm{NO}_{2}$ & $170 \mathrm{~s}, 140 \mathrm{~s}$ & $30 \mathrm{ppb}$ & $350 \%$ & 280 \\
\hline & $\mathrm{NH}_{3}$ & $11 \mathrm{~s}$ & $10 \mathrm{ppm}$ & $1.6 \%$ & 197 \\
\hline & $\mathrm{NH}_{3}$ & $40 \mathrm{~s}, 100 \mathrm{~s}$ & & $4.2 \%$ & 281 \\
\hline \multirow[t]{2}{*}{ Phosphorene } & $\mathrm{NO}_{2}$ & $30 \mathrm{~s}, 840 \mathrm{~s}$ & & $600 \%$ & 158 \\
\hline & $\mathrm{H}_{2} \mathrm{O}$ & & & $135 \% / \mathrm{RH}$ & 282 \\
\hline$h B N$ & $\mathrm{O}_{2}, \mathrm{CH}_{4}$ & $15 \mathrm{~s}, 20 \mathrm{~s}$ & & $7.8 \%\left(\mathrm{CH}_{4}\right)$ & 199 \\
\hline
\end{tabular}

List of abbreviations. ME : mechanically exfoliated, TEA : triethylamine, CVD : chemical vapour deposition, align. : aligned, EtOH : ethanol, LPE : liquid phase exfoliation, $\mathrm{MeOH}$ : methanol. 
By and large, in this first part of this Review article we have provided an overview on the recent developments in the application of the most common 2DMs as gas sensors. From the results highlighted it is possible to affirm that $2 \mathrm{DMs}$ are fully competitive materials for production of high-performance gas sensors with low operating temperature thanks to their peculiar properties. Among 2DMs, pristine graphene does not appear being ideal for assembling sensing devices because of its chemical inertness and zero-band gap, and thus lack of semiconducting properties. In fact, the modulation of the semiconductivity is a key characteristic to harness the performances of gas sensors. Furthermore, in pristine graphene the absence of dangling groups on its surface that act as bonding sites for the analytes hampers the gas absorption and the sensing performances. Towards this end, doping and modifying graphene with functional atoms and groups and assembly hybrid structures of graphene and functional nanomaterials have been proven to be the winning strategy for enhancement of gas sensing performance. A different approach relies on exploiting other 2DMs, which possess intrinsic functionality as TMDs $\left(\mathrm{MoS}_{2}, \mathrm{MoSe}_{2}, \mathrm{WS}_{2}\right.$ etc.). Unlike graphene, TMDs show remarkable semiconducting properties with a tuneable band gap, which depends on their thickness and on the doping effect of ad hoc molecules as the sensed gases. Therefore, TMDs are overall promising materials for the fabrication of gas sensors. Nevertheless, up to date, their (production) costs and the hurdle in the control over the thickness hinder their application in competitive gas sensing devices.

A few challenges still need to be tackled in order to make 2DMs competitive for the industrial sensing market. In particular, while the sensitivity has evidenced great improvement, an enhancement of response and recovery characteristics is necessary. In fact, in most of the reported examples 2DMs based gas sensors exhibit slow response and incomplete recovery when operating at room temperature. Additional performance parameters that require improvements are the selectivity and stability. Most of these materials show a poor selectivity for a cross-response to different sensed gases and in many cases the response signals are unstable. Such a modest selectivity can hardly find a definitive solution because of the major hurdle of supramolecular chemistry to develop highly selective receptors for volatile gas molecules. Finally, the fabrication methods often used as microfabrication and drop casting are not suitable for the industrial scale up for their complexity and time-demand. 


\section{Applications in metal sensing}

The rapid escalation of agricultural and industrial activities as a result of the population growth is yielding a dramatic proliferation of the amount of pollutants released daily worldwide into the environment. ${ }^{285-287}$ Metal ions in the aqueous environment have determined various diseases and seriously threaten ecosystem and public health with the rapid development of the industry in recent years. ${ }^{288,289}$ A great effort has been made to fabricate portable sensors for monitoring heavy metals in the environment. Within this framework also 2DMs-based materials have been integrated in different types of sensors capable of detecting heavy and alkali metal ions via electrical ${ }^{92,290-305}$ and optical outputs. ${ }^{306-317}$ 306-317 The interaction between 2DMs and metal ions have been extensively explored in the past years and as a result outstanding adsorption capabilities have been achieved, opening new avenues in the field of wastewater purification and sensing. ${ }^{89}$ Several excellent reviews have been published on the desalination of water using neat or functionalized $\mathrm{GO},{ }^{318-321}$ these reports discuss a variety of approaches to separate alkali metal ions from either seawater or wastewater through incorporation of GO or functionalized GO in membranes. Yet, those review articles do not discuss the use of $2 \mathrm{DMs}$ as platforms for sensing devices and in particular for detection of (heavy) metal ions. Many efforts have been made to develop new robust technologies for low-cost and effective portable sensors for monitoring heavy metals in the environment. Among various approaches, those based on physisorption or chemisorption, relying on the capturing of the pollutant (i.e. analyte) by an adsorbent (i.e. receptor), are chemically programmable as they exploit supramolecular recognition events. Understanding the dynamic adsorption capabilities of metal ions on the surface of 2DMs is therefore extremely important and will be discussed prior to the use of 2DMs in sensing devices for heavy metal ions.

\subsection{The adsorption process}

The development of ad hoc receptors of metal ions makes it possible to exploit the reversible processes of adsorption and desorption as extremely versatile strategies towards sensing in a variety of environments. Moreover, by relying on the reversible nature of non-covalent interactions, the sensor can exhibit a quick response, a fast recovery rate (i.e. real-time monitoring) and a facile regeneration to enable its use multiple times. 2DMs are atomically 
thick and possess two planar surfaces available for metal ion adsorption, thus featuring extremely high surface-to-volume ratios. In particular, GO is very interesting for the removal of metal ions due to its unique hydrophilic nature and the presence of functional groups containing oxygen atoms, which can efficiently bind the metal ions to form strong surface complexes. $^{78,322}$ On the other hand, TMDs intrinsically possess numerous chalcogen atoms, which can act as a potential coordination sites for certain heavy metal ions. The adsorption capabilities of $2 \mathrm{DMs}$ will be evaluated through the maximum adsorption capacity $\left(\mathrm{q}_{\max }\right)$ defined as the ratio between the maximum loaded mass of analyte expressed in milligrams and the mass of the absorbent expressed in grams.

\subsubsection{Metal sensing with graphene, graphene oxide and related composites}

Graphene-based materials are being considered as the most promising absorbents for capturing various heavy metal ions. ${ }^{323-325}$ Remarkably, graphene can be easily produced in the form of GO, which displays numerous oxygen-rich functional groups such as carbonyls, epoxides, and hydroxides that act both as reactive sites for further covalent functionalization, and can interact via dipole-dipole or strong electrostatic interactions with the metal ions, enhancing the occurrence of adsorption event. While several excellent reviews have been published on the desalination of water using neat or functionalized GO, ${ }^{318-321}$ these reports discuss a variety of approaches to separate alkali metal ions from either seawater or wastewater and do not discuss the abilities of other 2DMs as platforms to adsorb (heavy) metal ions. Conventional methods for the quantification of heavy metal ions include plasma mass spectrometry (ICP-AES), atomic absorption/emission spectroscopy (e.g. F-AAS, UVVIS), and polarography. To define the concentration of the remaining heavy metal ions after adsorption we should calculate the difference between the initial $\left(C_{0} ; \mathrm{mg} \mathrm{L}^{-1}\right)$ and the equilibrium $\left(C_{e} ; \mathrm{mg} \mathrm{L}^{-1}\right)$ concentration. The equilibrium sorption capacity and timedependent capacity were determined using Eq.2, where $\mathrm{q}_{\mathrm{e}}$ are the equilibrium amount of heavy metal ions adsorbed per unit mass $(\mathrm{m})$ of adsorbent $(\mathrm{mg} / \mathrm{g}), V$ is the volume of the metal ion solution.

$$
q_{e}=\frac{\left(C_{0}-C_{e}\right) \times V}{m_{\text {adsorbent }}}
$$

Among various adsorption isotherm models Freundlich and Langmuir are most commonly used for estimate the maximum adsorption capacity $\left(\mathrm{q}_{\max }\right)$ of metal ions on both $2 \mathrm{D}$ and $3 \mathrm{D}$ 
carbon-based adsorbents. ${ }^{322,324,326,327} \mathrm{GO}$ and chemically modified graphene oxide (CMGO) are considered as a promising adsorbents for the removal of heavy metal ions such as $\mathrm{Pb}(\mathrm{II})$, 78, 322, 326-356 $\mathrm{Cu}(\mathrm{II}),{ }^{78,327,335,337,339,347,349,351,356-366} \mathrm{Cr}(\mathrm{VI}),{ }^{262,286,337,355,367-377} \mathrm{Cd}(\mathrm{II}),{ }^{78,} 327$, 340, 349, $355 \mathrm{Hg}(\mathrm{II}),{ }^{335,349,355,378,379} \mathrm{Ni}(\mathrm{II}),{ }^{355,} 380 \mathrm{Co}(\mathrm{II}),{ }^{381} \mathrm{Mn}(\mathrm{II}),{ }^{327} \mathrm{Pd}(\mathrm{II}),{ }^{382} \mathrm{Sr}(\mathrm{II}),{ }^{383}$ $\mathrm{Au}(\mathrm{III}),{ }^{382} \mathrm{As}(\mathrm{V}),{ }^{384}$ and U(VI). ${ }^{385-387}$ Moreover, in order to increase the adsorption capacity and simplify the separation of the GO from water, numerous hybrid materials have been investigated and include combination of GO/CMGO with poly(acrylamide) (PAM), ${ }^{328}$, 331 poly $\left(N\right.$-vinylcarbazole) $(\mathrm{PVK})^{329}$, hyperbranched polyamine (HPA), ${ }^{330}$ iron oxide $\left(\mathrm{Fe}_{3} \mathrm{O}_{4}\right),{ }^{333}, 334,338$ polyamidoamine dendrimers (PAMAMs) ${ }^{327}$, ethylenediamine tetraacetic acid (EDTA), ${ }^{335,336}$ chitosan (CS), ${ }^{337,339,350,374,382}$ etc. Hybrids of GO with other polymers have also been used to remove organic contaminants from water. ${ }^{388}$

Among numerous factors affecting the adsorption capacity, initial concentration of the solution, phase contact time, temperature and $\mathrm{pH}$ are found to play a crucial role in the process of adsorption of metal ions on GO.

The adsorption properties of neat GO towards divalent metal ions (copper, zinc, cadmium and lead) were investigated by Sitko et al. ${ }^{78}$ In this seminal work, it was shown that GO prepared via the oxidation of synthetic graphite flakes using potassium dichromate possesses an impressive maximum adsorption capacity ( $\left.\mathrm{q}_{\max }\right)$ for $\mathrm{Cu}$ (II) $\left(223 \mathrm{mg} \mathrm{g}^{-1}\right), \mathrm{Zn}$ (II) (345 $\mathrm{mg} \mathrm{g}^{-}$ $\left.{ }^{1}\right), \mathrm{Cd}(\mathrm{II})$ (530 $\left.\mathrm{mg} \mathrm{g}^{-1}\right)$, and $\mathrm{Pb}(\mathrm{II})$ ions (1120 $\left.\mathrm{mg} \mathrm{g}^{-1}\right)$. The single and competitive adsorption of $\mathrm{Cu}(\mathrm{II}), \mathrm{Zn}(\mathrm{II}), \mathrm{Cd}(\mathrm{II})$ and $\mathrm{Pb}(\mathrm{II})$ shows that the affinities of GO for these metal ions follow the order of $\mathrm{Pb}(\mathrm{II})>\mathrm{Cu}(\mathrm{II})>>\mathrm{Cd}(\mathrm{II})>\mathrm{Zn}$ (II). Since then, many groups have investigated the impact of GO functionalization (with chemical groups and inorganic compounds) on its maximum adsorption capacity. The most relevant results reported so far have been listed in Table 3.

Table 3. Maximum adsorption capacities of $\mathrm{CMGO}$ composites used in $\mathrm{Pb}$ (II) removal process.

\begin{tabular}{|c|c|c|c|}
\hline Adsorbent & $q_{\max }\left(m g g^{-1}\right)$ & Conditions & Ref. \\
\hline \multirow{2}{*}{ Few layers $G O$} & $1850^{\mathrm{a}}$ & $\mathrm{pH}=6, \mathrm{~T}=333 \mathrm{~K}$ & 322 \\
\hline & $758^{\mathrm{a}}$ & $\mathrm{pH}=5.5, \mathrm{~T}=333$ & 332 \\
\hline$G O$ & $1119^{\mathrm{a}}$ & $\mathrm{pH}=5, \mathrm{~T}=298 \mathrm{~K}$ & 78 \\
\hline$r G O / P A M$ & $1000^{\mathrm{a}}$ & $\mathrm{pH}=6, \mathrm{~T}=298 \mathrm{~K}$ & 328 \\
\hline
\end{tabular}




\begin{tabular}{|c|c|c|}
\hline$P V K-G O$ & $888^{\mathrm{a}}$ & $\mathrm{pH}=7, \mathrm{~T}=298 \mathrm{~K}$ \\
\hline$H P A-G O$ & $820^{\mathrm{a}}$ & $\mathrm{pH}=5.9, \mathrm{~T}=318 \mathrm{~K}$ \\
\hline$P A M-G$ & $820^{\mathrm{a}}$ & $\mathrm{pH}=6, \mathrm{~T}=288 \mathrm{~K}$ \\
\hline $\mathrm{MnFe}_{2} \mathrm{O}_{4} / \mathrm{GO}$ & $673^{\mathrm{a}}$ & $\mathrm{pH}=5, \mathrm{~T}=298 \mathrm{~K}$ \\
\hline $\mathrm{GO} / \mathrm{Fe}_{3} \mathrm{O}_{4}$ & $588^{\mathrm{a}}$ & $\mathrm{pH}=5, \mathrm{~T}=303 \mathrm{~K}$ \\
\hline GO/PAMAMs & $568^{\mathrm{b}}$ & $\mathrm{pH}=6, \mathrm{~T}=283 \mathrm{~K}$ \\
\hline$E D T A / C M G O$ & $508^{\mathrm{a}}$ & $\mathrm{pH}=4.2, \mathrm{~T}=298 \mathrm{~K}$ \\
\hline$E D T A / G O$ & $479^{\mathrm{a}}$ & $\mathrm{pH}=6.8, \mathrm{~T}=298 \mathrm{~K}$ \\
\hline \multirow{2}{*}{$C S / G O$} & $461^{\mathrm{c}}$ & $\mathrm{pH}=6, \mathrm{~T}=318 \mathrm{~K}$ \\
\hline & $99^{\mathrm{a}}$ & $\mathrm{pH}=6, \mathrm{~T}=298 \mathrm{~K}$ \\
\hline $\mathrm{Fe}_{3} \mathrm{O}_{4} /$ Cysteine & $459^{\mathrm{a}}$ & $\mathrm{pH}=6, \mathrm{~T}=298 \mathrm{~K}$ \\
\hline$C S / G O-S H$ & $447^{\mathrm{b}}$ & $\mathrm{pH}=5, \mathrm{~T}=293 \mathrm{~K}$ \\
\hline Polydopamine/GO & $365^{\mathrm{a}}$ & $\mathrm{pH} \mathrm{NA}, \mathrm{T}=298 \mathrm{~K}$ \\
\hline $\mathrm{NH}_{2}-\mathrm{SiO}_{2} / \mathrm{GO}$ & $345^{\mathrm{a}}$ & $\mathrm{pH}=5, \mathrm{~T}=313 \mathrm{~K}$ \\
\hline Mesoporous silica/GO & $333^{\mathrm{a}}$ & $\mathrm{pH}=7.1, \mathrm{~T}=298 \mathrm{~K}$ \\
\hline $\mathrm{Ag} / \mathrm{GO}$ & $313^{\mathrm{a}}$ & $\mathrm{pH}=5.3, \mathrm{~T}=298 \mathrm{~K}$ \\
\hline Hydroxyapatite/GO & $278^{\mathrm{a}}$ & $\mathrm{pH}=4.5, \mathrm{~T}=308 \mathrm{~K}$ \\
\hline Polysiolxane/GO & $256^{\mathrm{a}}$ & $\mathrm{pH}=5, \mathrm{~T}=313 \mathrm{~K}$ \\
\hline $\begin{array}{l}\text { Phenylenediaminel } \\
r G O\end{array}$ & $228^{\mathrm{a}}$ & $\mathrm{pH}=7, \mathrm{~T}=298 \mathrm{~K}$ \\
\hline Tryptophan/GO & $222^{\mathrm{a}}$ & $\mathrm{pH}=4, \mathrm{~T}=293 \mathrm{~K}$ \\
\hline GO/Polyaniline & $217^{\mathrm{a}}$ & $\mathrm{pH}=5, \mathrm{~T}=303 \mathrm{~K}$ \\
\hline $\mathrm{CS} / \mathrm{FeOOH} / \mathrm{GO}$ & $111^{\mathrm{a}}$ & $\mathrm{pH}=5.5, \mathrm{~T}=313 \mathrm{~K}$ \\
\hline$G O-S H$ & $108^{\mathrm{a}}$ & $\mathrm{pH}=4-10, \mathrm{~T}=298 \mathrm{~K}$ \\
\hline $\mathrm{GO}-\mathrm{NH}_{2}$ & $96^{\mathrm{a}}$ & $\mathrm{pH}=6, \mathrm{~T}=298 \mathrm{~K}$ \\
\hline$M H C G O$ & $79^{\mathrm{a}}$ & $\mathrm{pH}=5.5, \mathrm{~T}=298 \mathrm{~K}$ \\
\hline$C M G O$ & $77^{\mathrm{a}}$ & $\mathrm{pH}=5, \mathrm{~T}=298 \mathrm{~K}$ \\
\hline $\mathrm{Fe}_{3} \mathrm{O}_{4}-\mathrm{G}$ & $28^{\mathrm{b}}$ & $\mathrm{pH}=6, \mathrm{~T}=293 \mathrm{~K}$ \\
\hline $\mathrm{NH}_{2}-\mathrm{SiO}_{2} / \mathrm{GO}$ & $14^{\mathrm{a}}$ & $\mathrm{pH}=5.5, \mathrm{~T}=298 \mathrm{~K}$ \\
\hline
\end{tabular}

List of abbreviations. rGO : reduced graphene oxide, PAM : poly(acrylamide), PVK : Poly( $N$-vinylcarbazole), HPA : hyperbranched polyamine, $\mathrm{MnFe}_{2} \mathrm{O}_{4}$ : manganese iron oxide, $\mathrm{Fe}_{3} \mathrm{O}_{4}$ : iron oxide, PAMAM : polyamidoamine, EDTA : Ethylenediaminetetraacetic acid, CMGO : chemically modified graphene oxide, CS : chitosan, GO-SH : Sulfydryl-functionalized graphene oxide, $\mathrm{NH}_{2}-\mathrm{SiO}_{2}$ : amino siloxane, $\mathrm{FeOOH}$ : iron (III) oxide-hydroxide, GO-NH : aminosilanized graphene oxide, MHCGO : magnetic carboxymethyl chitosan. $\mathrm{Q}_{\max }$ values were calculated using (a) Langmuir, (b) Freundlich or (c) Redlich Peterson isotherms.

Zhao et al. demonstrated that $\mathrm{q}_{\max }$ of two- or three-layer thick GO nanosheets synthesized from flake-graphite through modified Hummers method can be tuned with temperature. ${ }^{322}$ 
Interestingly, it has been shown that the adsorption of $\mathrm{Pb}(\mathrm{II})$ ions on few-layer thick GO nanosheets was independent of the ionic strength, i.e. not affected by concentrations of background electrolyte $\left(\mathrm{NaClO}_{4}\right)$ at the $\mathrm{pH}$ ranging from 1 to 13 . In particular, $\mathrm{q}_{\max }$ of $\mathrm{Pb}(\mathrm{II})$ ions calculated from the Langmuir model amount to 842,1150 , and $1850 \mathrm{mg} \mathrm{g}^{-1}$ at 293, 313, and $333 \mathrm{~K}$, respectively. Yang et al. proposed a different approach which relies on the functionalization of rGO with water-soluble poly(acrylamide) (PAM). ${ }^{328}$ The GO prepared following the Staudenmaier method was thermally reduced yielding rGO. PAM is a polymer with a large number of acetylamine groups in its macromolecular chains, which can interact with metal ions via coordination. In particular, the carboxyl groups at the periphery of rGO sheets were converted to amine groups by reaction with $\mathrm{N}$-hydroxysuccinimide and 1,3diaminopropane, and a free-radical polymerization initiator was anchored to the rGO sheets. The highest adsorption capacities of rGO/PAM for $\mathrm{Pb}(\mathrm{II})$ amounted to $1000 \mathrm{mg} \mathrm{g}^{-1}$ (298 K), which is comparable to the neat rGO.

Aqueous solutions containing copper ions have been exploited for technological applications in the fields of mechanical manufacturing, electroplating, light industry and architecture. Yet, these solutions may cause serious diseases in the human central nervous system. ${ }^{361}$ According to EPA regulations, copper concentration in drinking water should not exceed $1300 \mathrm{ng} \mathrm{mL}^{-}$ 1351 Tan et al. prepared a hybrid composite including L-tryptophan (L-Trp) and GO by nucleophilic substitution reaction in order to increase the hydrophobicity of GO and to promote the sorption. ${ }^{347}$ It was found that such chemical modification of GO increases its sorption capacity from 223 to $588 \mathrm{mg} \mathrm{g}^{-1}$. It has been shown that that high removal efficiencies can be obtained reaching values exceeding $95 \%$ at $\mathrm{pH} 5$ and 4 for $\mathrm{Cu}(\mathrm{II})$ and $\mathrm{Pb}(\mathrm{II})$, respectively

Several examples of CMGO-based adsorbents rely on the use of chitosan as molecule possessing a high affinity for heavy metal ions. ${ }^{337,339,350,374,382}$ Chitosan reacts with GO carboxyl groups of GO and forms amide bonds. ${ }^{262,354}$ In particular, chitosan modified GO has been prepared via covalent modification and electrostatic self-assembly by Li et al. ${ }^{339}$ The introduction of GO-SH sheets as an interlayer can offer extra space into the chitosan structure and further increase the specific surface area. The results indicated that a new type of sorbent material, with functional groups such as $-\mathrm{OH},-\mathrm{COOH},-\mathrm{SH}$ and $-\mathrm{NH}_{2}$ has a high adsorption capacity of copper ions (425 $\mathrm{mg} \mathrm{g}^{-1}$, see Table 4). Furthermore, the recyclability of the sorbent has been studied by treating it with $\mathrm{HNO}_{3}$ and EDTA solutions. The chitosan (CS)/GO-SH revealed a decrease of the adsorption capacity over three cycles by $23 \%$ for $\mathrm{Cu}$, 
$25 \%$ for $\mathrm{Pb}$ and $26 \%$ for $\mathrm{Cd}$ ions. Chitosan/GO hybrid in the form of nanofibrous composite has been studied by Najafabadi et al. ${ }^{337}$ The nanofibrous morphology of the hybrid material has been achieved through the use of electrospinning process. The maximum $\mathrm{Cu}$ (II) adsorption capacity was estimated as $423.8 \mathrm{mg} \mathrm{g}^{-1}$. It was shown that the $\mathrm{q}_{\max }$ decreased slowly with the increasing cycle number; such behaviour was attributed to the decrease in availability of active sites of adsorbent for metal ions. CS/GO-SH nanofibers could be used up to the fifth cycle of regeneration using $\mathrm{HNO}_{3}$ solution by retaining $91.5 \%$ of the initial adsorption capacity $\mathrm{Cu}(\mathrm{II})$ ions sorption.

Table 4. Maximum adsorption capacities of $\mathrm{CMGO}$ composites used in $\mathrm{Cu}(\mathrm{II})$ removal process.

\begin{tabular}{|c|c|c|c|}
\hline Adsorbent & $q_{\max }\left(m g g^{-1}\right)$ & Conditions & Ref. \\
\hline $\operatorname{Trp} / G O$ & $588^{\mathrm{a}}$ & $\mathrm{pH}=5, \mathrm{~T}=293 \mathrm{~K}$ & 347 \\
\hline \multirow{5}{*}{$C S / G O$} & $425^{\mathrm{a}}$ & $\mathrm{pH}=5, \mathrm{~T}=293 \mathrm{~K}$ & 339 \\
\hline & $424^{\mathrm{c}}$ & $\mathrm{pH}=6, \mathrm{~T}=318 \mathrm{~K}$ & 337 \\
\hline & $203^{b}$ & $\mathrm{pH}=5, \mathrm{~T}=293 \mathrm{~K}$ & 358 \\
\hline & $162^{\mathrm{a}}$ & $\mathrm{pH}=5.5, \mathrm{~T}=303 \mathrm{~K}$ & 359 \\
\hline & $54^{\mathrm{a}}$ & $\mathrm{pH}=5, \mathrm{~T}=293 \mathrm{~K}$ & 366 \\
\hline Polyallylamine/GO & $349^{\mathrm{a}}$ & $\mathrm{pH}=6, \mathrm{~T}=293 \mathrm{~K}$ & 357 \\
\hline$E D T A / C M G O$ & $301^{\mathrm{a}}$ & $\mathrm{pH}=5, \mathrm{~T}=298 \mathrm{~K}$ & 335 \\
\hline \multirow{2}{*}{$G O$} & $294^{\mathrm{a}}$ & $\mathrm{pH}=5, \mathrm{~T}=298 \mathrm{~K}$ & 78 \\
\hline & $117^{\mathrm{b}}$ & $\mathrm{pH}=5.3, \mathrm{~T}=293 \mathrm{~K}$ & 361 \\
\hline$G O / C d S$ & $137^{\mathrm{a}}$ & $\mathrm{pH}=6, \mathrm{~T}=298 \mathrm{~K}$ & 360 \\
\hline$G O-E D T A$ & $109^{\mathrm{a}}$ & $\mathrm{pH}=5, \mathrm{~T}=293 \mathrm{~K}$ & 362 \\
\hline $\mathrm{GO}-\mathrm{NH}_{2}$ & $103^{\mathrm{a}}$ & $\mathrm{pH}=6, \mathrm{~T}=298 \mathrm{~K}$ & 363 \\
\hline \multirow{2}{*}{$G O-S H$} & $100^{\mathrm{a}}$ & $\mathrm{pH}=6, \mathrm{~T}=298 \mathrm{~K}$ & 363 \\
\hline & $42^{\mathrm{a}}$ & $\mathrm{pH}=5-10, \mathrm{~T}=293 \mathrm{~K}$ & 351 \\
\hline GO/PAMAMs & $69^{\mathrm{a}}$ & $\mathrm{pH}=4.5, \mathrm{~T}=298 \mathrm{~K}$ & 327 \\
\hline$S M G O$ & $63^{\mathrm{a}}$ & $\mathrm{pH}=4.7, \mathrm{~T}=323 \mathrm{~K}$ & 364 \\
\hline Alginate/GO & $60^{\mathrm{a}}$ & $\mathrm{pH}=\mathrm{NA}, \mathrm{T}=293 \mathrm{~K}$ & 365 \\
\hline $\mathrm{NH}_{2}-\mathrm{SiO}_{2} / \mathrm{GO}$ & $6^{a}$ & $\mathrm{pH}=5.5, \mathrm{~T}=293 \mathrm{~K}$ & 356 \\
\hline
\end{tabular}

List of abbreviations. Trp : tryptophan, CS : chitosan, EDTA : Ethylenediaminetetraacetic acid, CMGO : chemically modified graphene oxide, CdS : cadmium sulfide, $\mathrm{GO}-\mathrm{NH}_{2}$ : aminosilanized graphene oxide, GO-SH : Sulfydrylfunctionalized graphene oxide PAMAM : polyamidoamine, $\mathrm{SMGO}$ : sulfonated magnetic graphene oxide , $\mathrm{NH}_{2}-\mathrm{SiO}_{2}$ : amino siloxane.

$\mathrm{Q}_{\max }$ values were calculated using (a) Langmuir, (b) Freundlich or (c) Redlich Peterson isotherms. 
Chromium, which exists most frequently as $\mathrm{Cr}(\mathrm{III})$ and $\mathrm{Cr}(\mathrm{VI}),{ }^{377}$ is commonly used in metallurgy, tanning, military, dyes and pigments. ${ }^{368,} 369$ Generally, $\mathrm{Cr}(\mathrm{VI})$ compounds are considered to be more toxic than $\mathrm{Cr}(\mathrm{III})$ because of their toxicity to humans, animals, plants and microorganisms. ${ }^{377,389}$ A variety of chemical approaches has been employed in order to increase the affinity of GO towards chromium ions. For example, functionalization of GO with polypyrrole (PPy), being a widely studied conductive polymer, has been exploited by Li et $a l .{ }^{368}$ The PPy/GO composite nanosheets prepared by sacrificial template polymerization method exhibit adsorption capacity for $\mathrm{Cr}(\mathrm{VI})$ ions as high as $497 \mathrm{mg} \mathrm{g}^{-1}$. Moreover, the adsorption capacity of the PPy/GO composite nanosheets is about twice as large as that of conventional PPy nanoparticles. ${ }^{390,} 391$ Supplementary studies showed that subsequent covalent functionalization of $\mathrm{PPy} / \mathrm{GO}$ with $\alpha$-cyclodextrin ${ }^{367}$ leads to maximum adsorption capacities of $606 \mathrm{mg} \mathrm{g}^{-1}$ at $25^{\circ} \mathrm{C}$ and $667 \mathrm{mg} \mathrm{g}^{-1}$ at $45^{\circ} \mathrm{C}$.

Table 5. Maximum adsorption capacities of CMGO composites used in $\mathrm{Cr}(\mathrm{VI})$ removal process.

\begin{tabular}{|c|c|c|c|}
\hline Adsorbent & $q_{\max }\left(m g g^{-1}\right)$ & Conditions & Ref. \\
\hline$P P y / c y c l o d e x t r i n / G O$ & $667^{\mathrm{a}}$ & $\mathrm{pH}=2, \mathrm{~T}=318 \mathrm{~K}$ & 367 \\
\hline$P P y / G O$ & $497^{\mathrm{a}}$ & $\mathrm{pH}=3, \mathrm{~T}=293 \mathrm{~K}$ & 368 \\
\hline \multirow{4}{*}{$C S / G O$} & $310^{\mathrm{c}}$ & $\mathrm{pH}=3, \mathrm{~T}=318 \mathrm{~K}$ & 337 \\
\hline & $219^{\mathrm{a}}$ & $\mathrm{pH}=2, \mathrm{~T}=293 \mathrm{~K}$ & 371 \\
\hline & $145^{\mathrm{a}}$ & $\mathrm{pH}=3-4, \mathrm{~T}=303 \mathrm{~K}$ & 374 \\
\hline & $108^{\mathrm{a}}$ & $\mathrm{pH}=2, \mathrm{~T}=303 \mathrm{~K}$ & 376 \\
\hline $\mathrm{Fe}_{3} \mathrm{O}_{4} / \mathrm{GO}$ & $259^{\mathrm{b}}$ & $\mathrm{pH}=2, \mathrm{~T}=298 \mathrm{~K}$ & 369 \\
\hline Trioctylamine/GO & $232^{\mathrm{a}}$ & $\mathrm{pH}=2.5-3, \mathrm{~T}=293 \mathrm{~K}$ & 370 \\
\hline$G-M g A l-L D H$ & $172^{\mathrm{b}}$ & $\mathrm{pH}=2, \mathrm{~T}=293 \mathrm{~K}$ & 372 \\
\hline $\mathrm{Fe} / \mathrm{G}$ & $162^{\mathrm{a}}$ & $\mathrm{pH}=4.5, \mathrm{~T}=293 \mathrm{~K}$ & 373 \\
\hline Cyclodextrin/GO & $120^{\mathrm{a}}$ & $\mathrm{pH}=3, \mathrm{~T}=293 \mathrm{~K}$ & 286 \\
\hline $\mathrm{Fe}_{3} \mathrm{O}_{4} / \mathrm{TiO}_{2} / \mathrm{GO}$ & $118^{\mathrm{b}}$ & $\mathrm{pH}=2, \mathrm{~T}=303 \mathrm{~K}$ & 375 \\
\hline$D C T A / G O$ & $84^{\mathrm{b}}$ & $\mathrm{pH}=2, \mathrm{~T}=303 \mathrm{~K}$ & 377 \\
\hline $\mathrm{Fe}_{3} \mathrm{O}_{4} / \mathrm{G}$ & $17^{\mathrm{b}}$ & $\mathrm{pH}=1-3, \mathrm{~T}=293 \mathrm{~K}$ & 355 \\
\hline
\end{tabular}

List of abbreviations. PPy : polypyrrole, CS : chitosan, $\mathrm{Fe}_{3} \mathrm{O}_{4}$ : iron oxide, $\mathrm{TiO}_{2}$ : titanum dioxide, G-MgAl-LDH : graphene - magnesium/aluminum - layered double hydroxide, DCTA : 1,2-diamino cyclohexanetetraacetic acid. $\mathrm{Q}_{\max }$ values were calculated using (a) Langmuir, (b) Freundlich or (c) Redlich Peterson isotherms. 
Numerous organic and inorganic compounds have been used to functionalize GO and form composites in order to detect and capture other heavy metal ions like $\mathrm{Cd}(\mathrm{II}),{ }^{78,327,340,349,355}$ $\mathrm{Hg}(\mathrm{II}),{ }^{335,349,378,379} \mathrm{Ni}(\mathrm{II}),{ }^{355,380} \mathrm{Co}(\mathrm{II}),{ }^{381} \mathrm{Mn}(\mathrm{II}),{ }^{327} \mathrm{Pd}(\mathrm{II}),{ }^{382} \mathrm{Sr}(\mathrm{II}),{ }^{383} \mathrm{Au}(\mathrm{III}),{ }^{382} \mathrm{As}(\mathrm{II}){ }^{384}$ and U(III) ${ }^{385-387}$ efficiently from aqueous solutions. Zhang et al. have demonstrated that efficient CMGO-based sorbent can be achieved by decorating GO with polyamidoamine dendrimers (GO/PAMAMs). ${ }^{327}$ The adsorption behaviour of GO/PAMAMs for heavy metal ions in water solution was studied by changing the concentration of heavy metal ions, $\mathrm{pH}$ values, and temperature. The maximum adsorption capacities of GO/PAMAMs were found to be 568.18, 253.81, 68.68, and $18.29 \mathrm{mg} \mathrm{g}^{-1}$ for $\mathrm{Pb}(\mathrm{II}), \mathrm{Cd}(\mathrm{II}), \mathrm{Cu}(\mathrm{II})$, and $\mathrm{Mn}(\mathrm{II})$, respectively. Noteworthy, it was also found that adsorption capacities of the GO/PAMAMs for the heavy metal ions were highly $\mathrm{pH}$ dependent. In particular, at $\mathrm{pH}<3$, the hydronium ions of higher concentration compete with $\mathrm{M}$ (II) to grasp the adsorption sites. With an increase of $\mathrm{pH}$, the protonation degree of the amino groups was weakened, and the coordination and chelating ability of PAMAM's amino groups toward $\mathrm{Pb}(\mathrm{II}), \mathrm{Cd}(\mathrm{II}), \mathrm{Cu}(\mathrm{II})$, and $\mathrm{Mn}(\mathrm{II})$ reinforced.

Conducting polymers interfaced with carbon and carbon-based derivatives have displayed enhanced removal of mercury and other toxic materials from water. A facile chemical route was reported by Chandra et al. ${ }^{378}$ In particular, they showed that polypyrrole (PPy)-rGO composites possess a highly selective $\mathrm{Hg}^{2+}$ removal capacity. rGO sheets cross-linked with polypyrrole exhibited an increased surface area of $166 \mathrm{~m}^{2} \mathrm{~g}^{-1}$. The uptake of $\mathrm{Hg}^{2+}$ by $\mathrm{PPy} / \mathrm{rGO}$ has been estimated being as high as $980 \mathrm{mg} \mathrm{g}^{-1}$. Furthermore, PPy/rGO possess an extremely high desorption capacity of up to $92.3 \%$. Chitosan/GO (CSGO) composites with three different loadings of GO, i.e. 5, 10 and $15 \mathrm{wt} \%$ were prepared for the adsorption of $\mathrm{Au}(\mathrm{III})$ and $\mathrm{Pd}(\mathrm{II})$ by Liu et al. ${ }^{382}$ The adsorption capacity of $\mathrm{Au}(\mathrm{III})$ and $\mathrm{Pd}(\mathrm{II})$ onto CSGO composites revealed high values at $\mathrm{pH} 3.0-5.0$ for $\mathrm{Au}(\mathrm{III})$ and $\mathrm{pH} 3.0-4.0$ for $\mathrm{Pd}(\mathrm{II})$. It was found, that the composite with $5 \mathrm{wt} \%$ of GO had the largest adsorption capacity for $\mathrm{Au}(\mathrm{III})$ and $\mathrm{Pd}(\mathrm{II})$ compared with the other prepared adsorbents, where the maximum adsorption capacity amounted to $1076.6 \mathrm{mg} \mathrm{g}^{-1}$ and $216.9 \mathrm{mg} \mathrm{g}^{-1}$ for $\mathrm{Au}(\mathrm{III})$ and $\mathrm{Pd}(\mathrm{II})$, respectively.

Noteworthy, GO-based composites can also be used also in adsorption process of radioactive ions. $^{385-387}$ Chen et al. reported on the amino functionalized magnetic graphene oxide composite (AMGO) synthesized by a facile, one-step solvothermal method, tailor made for the U(VI) removal from aqueous solutions. ${ }^{387}$ It was shown, that the sorption of U(VI) on 
AMGO occurs via the formation of coordination complexes with the nitrogen- and oxygencontaining functional groups. It was concluded that the chemical affinity of the U(VI) for the nitrogen containing functional groups is stronger than that for the oxygen containing functional groups. Interestingly, the AMGO composite could be recovered from the solution with the magnetic separation within one minute. The same group has demonstrated that the adsorption capacity of CMGO towards uranium can be improved by functionalizing GO with activated carbon felt (ACF) through electrophoretic deposition and subsequent thermal annealing. ${ }^{385}$ The $\mathrm{q}_{\max }$ of GO-ACF for $\mathrm{U}(\mathrm{VI})$ amounted to $298 \mathrm{mg} \mathrm{g}^{-1}$ at $\mathrm{pH} 5.5$, being much higher than the one of ACF (173 $\mathrm{mg} \mathrm{g}^{-1}$ ), thus suggesting that the carboxyl functional groups of GO-ACF play an salient role in the sorption process, yielding a high efficiency for the removal of $\mathrm{U}(\mathrm{VI})$.

Table 6. Maximum adsorption capacities of CMGO composites used in various metal ions removal process.

\begin{tabular}{|c|c|c|c|c|}
\hline Adsorbent & Contaminant & $q_{\max }\left(m g g^{-1}\right)$ & Conditions & Ref. \\
\hline$G O$ & \multirow{4}{*}{$\mathrm{Cd}(\mathrm{II})$} & $530^{\mathrm{a}}$ & $\mathrm{pH}=5, \mathrm{~T}=298 \mathrm{~K}$ & 78 \\
\hline GO/PAMAMs & & $253^{\mathrm{b}}$ & $\mathrm{pH}=5, \mathrm{~T}=298 \mathrm{~K}$ & 327 \\
\hline$P D A / G O$ & & $210^{\mathrm{a}}$ & $\mathrm{pH}=\mathrm{NA}, \mathrm{T}=298 \mathrm{~K}$ & 340 \\
\hline $\mathrm{Fe}_{3} \mathrm{O}_{4} / \mathrm{G}$ & & $28^{\mathrm{b}}$ & $\mathrm{pH}=6-7, \mathrm{~T}=293 \mathrm{~K}$ & 355 \\
\hline$P P y-r G O$ & \multirow{4}{*}{$\mathrm{Hg}(\mathrm{II})$} & $980^{\mathrm{a}}$ & $\mathrm{pH}=3, \mathrm{~T}=293 \mathrm{~K}$ & 378 \\
\hline Ferrite/CS/G & & $361^{\mathrm{a}}$ & $\mathrm{pH}=7, \mathrm{~T}=323 \mathrm{~K}$ & 379 \\
\hline$E D T A-G O$ & & $268^{\mathrm{b}}$ & $\mathrm{pH}=4.1, \mathrm{~T}=298 \mathrm{~K}$ & 335 \\
\hline $\mathrm{Fe}_{3} \mathrm{O}_{4^{-}} \mathrm{G}$ & & $23^{\mathrm{b}}$ & $\mathrm{pH}=6-7, \mathrm{~T}=293 \mathrm{~K}$ & 355 \\
\hline$G O-G$ & \multirow{2}{*}{$\mathrm{Ni}(\mathrm{II})$} & $37^{\mathrm{a}}$ & $\mathrm{pH}=6, \mathrm{~T}=293 \mathrm{~K}$ & 380 \\
\hline $\mathrm{Fe}_{3} \mathrm{O}_{4^{-}} \mathrm{G}$ & & $22^{\mathrm{b}}$ & $\mathrm{pH}=6-7, \mathrm{~T}=293 \mathrm{~K}$ & 355 \\
\hline $\mathrm{GO}-\mathrm{NH}_{2}$ & $\mathrm{Co}(\mathrm{II})$ & $116^{\mathrm{a}}$ & $\mathrm{pH}=6, \mathrm{~T}=298 \mathrm{~K}$ & 381 \\
\hline GO/PAMAMs & $\mathrm{Mn}(\mathrm{II})$ & $18^{\mathrm{a}}$ & $\mathrm{pH}=4, \mathrm{~T}=298 \mathrm{~K}$ & 327 \\
\hline$C S / G O$ & $\operatorname{Pd}(\mathrm{II})$ & $216^{\mathrm{a}}$ & $\mathrm{pH}=3, \mathrm{~T}=323 \mathrm{~K}$ & 382 \\
\hline$P A M / G O$ & $\operatorname{Sr}(\mathrm{II})$ & $185^{\mathrm{a}}$ & $\mathrm{pH}=8.5, \mathrm{~T}=303 \mathrm{~K}$ & 383 \\
\hline$C S / G O$ & $\mathrm{Au}(\mathrm{III})$ & $1076^{\mathrm{a}}$ & $\mathrm{pH}=4, \mathrm{~T}=303 \mathrm{~K}$ & 382 \\
\hline $\mathrm{GO}-\mathrm{FeOOH}$ & $\operatorname{As}(\mathrm{V})$ & $73^{\mathrm{a}}$ & $\mathrm{pH}=7, \mathrm{~T}=298 \mathrm{~K}$ & 384 \\
\hline$G O / A C$ & \multirow{3}{*}{$\mathrm{U}(\mathrm{VI})$} & $298^{\mathrm{a}}$ & $\mathrm{pH}=5, \mathrm{~T}=298 \mathrm{~K}$ & 385 \\
\hline GO-sepiolite & & $161^{\mathrm{a}}$ & $\mathrm{pH}=5, \mathrm{~T}=298 \mathrm{~K}$ & 386 \\
\hline $\mathrm{GO}-\mathrm{NH}_{2}$ & & $141^{\mathrm{a}}$ & $\mathrm{pH}=6, \mathrm{~T}=298 \mathrm{~K}$ & 387 \\
\hline
\end{tabular}


List of abbreviations. PAMAM : polyamidoamine, PDA: polydopamine, $\mathrm{Fe}_{3} \mathrm{O}_{4}$ : iron oxide, $\mathrm{PPy}$ : polypyrrole, $\mathrm{CS}$ : chitosan, EDTA: Ethylenediaminetetraacetic acid, GO- $\mathrm{NH}_{2}$ : aminosilanized graphene oxide, PAM : poly(acrylamide), $\mathrm{FeOOH}$ : iron (III) oxide-hydroxide, AC : activated carbon.

$\mathrm{Q}_{\max }$ values were calculated using (a) Langmuir or (b) Freundlich isotherms.

\subsubsection{Metal sensing with transition metal dichalcogenides}

TMDs are characterized by an abundance of intrinsic chalcogen atoms, which are suitable coordination sites for certain heavy metal ions. Because of this reason, TMDs are now being considered as promising sorbents. In particular, due to the strong non-covalent sulphurmercury and sulphur-lead interactions $\mathrm{MoS}_{2}$ has been recently investigated for the efficient purification of wastewater. The thickness of the S-Mo-S triple layer in $\mathrm{MoS}_{2}$ amounts to 3.17 $\AA$, resulting in the distance of $2.98 \AA$ between two neighbouring layers. ${ }^{140}$ Such a distance is too narrow to allow the penetration of hydrated chalcogen ions in the interior spaces where the vast majority of potential coordination sites are located. ${ }^{392}$ Therefore, maximizing the number of accessible sulphur atoms is a crucial step for using layered TMDs in the highefficiency removal of heavy metal ions from contaminated water. Numerous papers have been published on the control over the interlayer spacing of $\mathrm{MoS}_{2}$, either by post-treatment of bulk $\mathrm{MoS}_{2}$ or in situ synthesis. For example, Gao et al. reported the preparation of $\mathrm{MoS}_{2}$ with interlayer spacing of $9.4 \AA$ via the intercalation of oxidized DMF species. ${ }^{393}$ Zheng et al. demonstrated that the interlayer spacing of $\mathrm{MoS}_{2}$ could be increased to $7.08 \AA, 8.99 \AA$, and $11.61 \AA$ by intercalation with $\mathrm{Li}^{+}, \mathrm{K}^{+}$and $\mathrm{Na}^{+}$, respectively. ${ }^{394}$ Recently, Ai et al. showed that the interlayer spacing of $\mathrm{MoS}_{2}$ can also be controlled by the synthesis temperatures. ${ }^{395}$ In particular, the interlayer spacing of the obtained $\mathrm{MoS}_{2}$ was greatly enlarged (9.5 $\AA$ ) in the temperature range of $140-200{ }^{\circ} \mathrm{C}$; upon further increase of the temperature up to $220{ }^{\circ} \mathrm{C}$, the interlayer spacing of the obtained $\mathrm{MoS}_{2}$ was found to be almost identical to that of bulk $\mathrm{MoS}_{2}$. Additionally, extremely fast adsorption kinetics during efficiently reduced the mercury concentration have been observed (Figure 11a). In brief, $20 \mathrm{mg}$ widened defect-rich nano $\mathrm{MoS}_{2}$ (W-DR-N-MoS 2 ) was added to $200 \mathrm{~mL}$ of aqueous solution containing $10000 \mathrm{ppb}$ $\mathrm{Hg}^{2+}$. The concentration of $\mathrm{Hg}^{2+}$ drastically decreased to $16.9 \mathrm{ppb}$ after 5 min of treatment, indicating a removal efficiency of $99.83 \%$. The structural features were systematically investigated of W-DR-N-MoS 2 (Figure 11b-e). They revealed for the first time that such widened defect-rich $\mathrm{MoS}_{2}$ (W-DR-N-MoS 2 ) nanosheets are capable to capture $\mathrm{Hg}$ (II) ions, with an extremely high $\mathrm{q}_{\max }\left(2563 \mathrm{mg} \mathrm{g}^{-1}\right)$ (Figure 11f) closely matching the theoretically 
predicted value of $2506 \mathrm{mg} \mathrm{g}^{-1}$ (assuming a stoichiometric $\mathrm{S} / \mathrm{Hg}$ ratio of 1:1), being higher than the $\mathrm{q}_{\max }$ of the best adsorbents to date. ${ }^{396-398}$ Selectivity of W-DR-N-MoS 2 exhibited negligible capturing capability for various competitive ions, such as $\mathrm{Na}^{+}, \mathrm{K}^{+}, \mathrm{Ca}^{2+}, \mathrm{Mg}^{2+}$, $\mathrm{Al}^{3+}, \mathrm{Cr}^{3+}, \mathrm{Mn}^{2+}, \mathrm{Zn}^{2+}$ and $\mathrm{Cd}^{2+}$, and even inferior capturing capacity for $\mathrm{Fe}^{3+}, \mathrm{Cu}^{2+}$ and $\mathrm{Pb}^{2+}$.
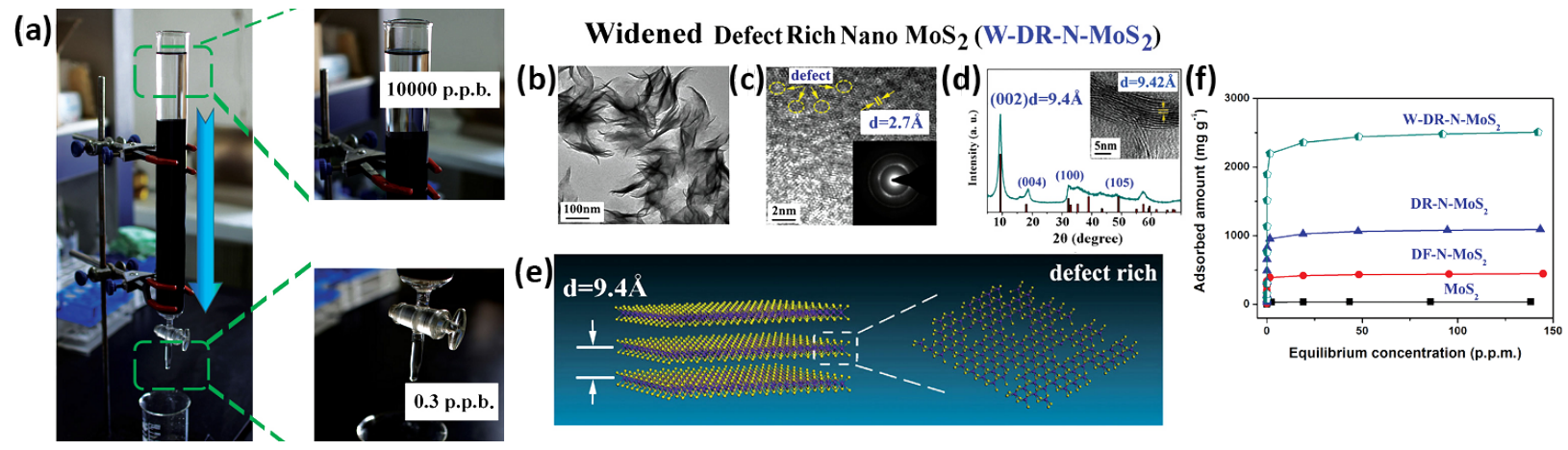

Figure 11. (a) Purification of natural water sample. The mercury-contaminated water was passed through a purification column filled with W-DR-N-MoS 2 : (b) TEM image of W-DR$\mathrm{N}-\mathrm{MoS}_{2}$. (c) HRTEM image of the basal plane of W-DR-N-MoS 2 , inset is the corresponding SAED pattern. (d) XRD pattern and the cross-sectional HRTEM image of W-DR-N-MoS ${ }_{2}$. (e) Structural model of W-DR-N-MoS 2 with enlarged interlayer spacing and multiple defects on the basal planes (The sulphur atoms are yellow and the Mo atoms are purple). (f) Mercury adsorption isotherm, compared with commercial $\mathrm{MoS}_{2}$ powder, DF-N-MoS 2 and DR-N$\mathrm{MoS}_{2}$. Adapted from Ref. ${ }^{395}$ with permission of Wiley-VCH.

Liu et al. showed that $\mathrm{MoS}_{2}$ exhibits superior ability to adsorb lead ions. ${ }^{399} \mathrm{MoS}_{2}$ nanosheets were prepared with an ultrasound assisted electrochemical exfoliation method. As in the case of $\mathrm{Hg}$ (II) ions the adsorption capacity of $\mathrm{MoS}_{2}$ towards $\mathrm{Pb}$ (II) can be attributed to the coordination of $\mathrm{Pb}(\mathrm{II})$ with $\mathrm{S}$ atoms of $\mathrm{MoS}_{2}$. Moreover, the strength of such non-covalent interactions is being reinforced by electrostatic adsorption. The experimental $\mathrm{Pb}(\mathrm{II})$ uptake capacity of $\mathrm{MoS}_{2}$ was estimated being as high as $1479 \mathrm{mg} \mathrm{g}^{-1}$. The adsorption followed the Freundlich isotherm model and fitted well with both the pseudo-first-order and pseudosecond-order kinetics models. The adsorption of $\mathrm{Pb}(\mathrm{II})$ on $\mathrm{MoS}_{2}$ materials was further confirmed by the measurement of SEM-EDS (Figure 12a-d) and XPS measurements (Figure 12e). The immobilization of heavy metal ions on $2 \mathrm{DMs}$ is depicted on Figure 12f. The adsorption might be attributed to the chemical adsorption due to the complexation of $\mathrm{Pb}(\mathrm{II})$ with intrinsic $\mathrm{S}$ or $\mathrm{O}$ atoms exposed on $\mathrm{MoS}_{2}$ surfaces, together with electrostatic adsorption. 

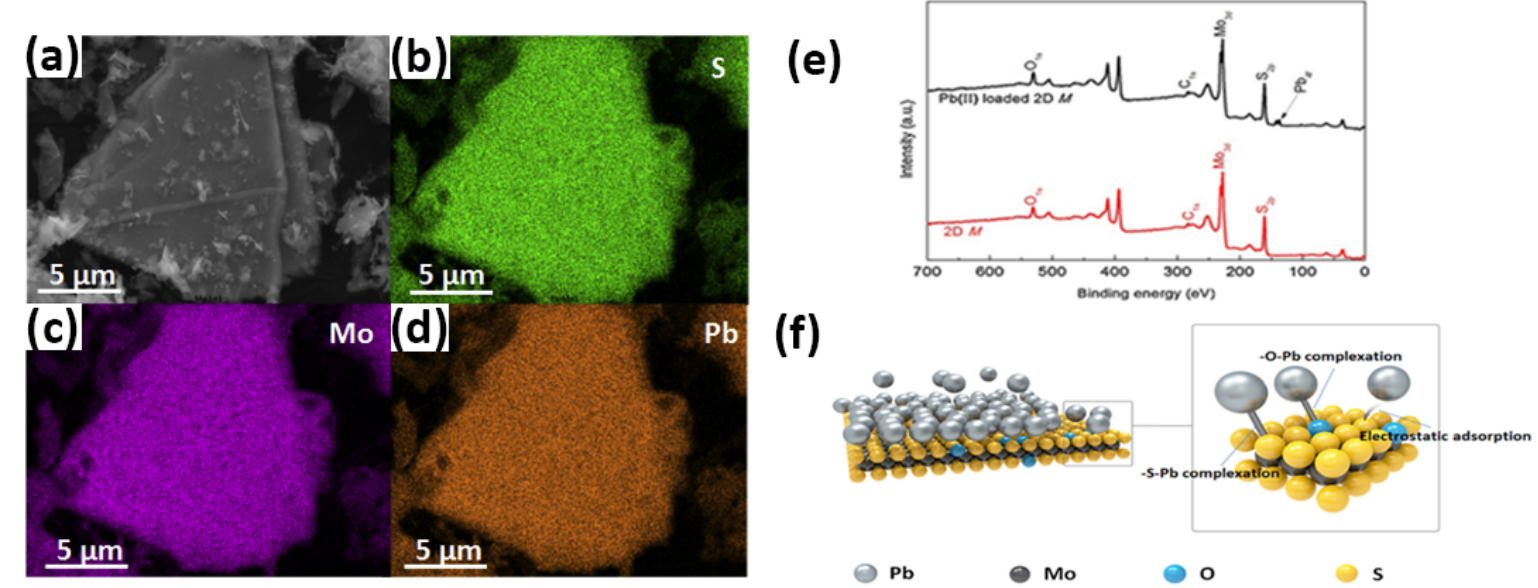

Figure 12. (a) SEM image of $\mathrm{Pb}(\mathrm{II})$ loaded 2DM. b-d) SEM-EDS elemental mapping images of $\mathrm{S}, \mathrm{Mo}$, and $\mathrm{Pb}$, respectively. (e) Diagrammatic illustration of the mechanism for $\mathrm{Pb}(\mathrm{II})$ adsorption on 2DM. (f) XPS survey spectra of 2DM before and after $\mathrm{Pb}$ (II) adsorption. Adapted from Ref. ${ }^{399}$ with permission of Elsevier Ltd.

Other studies showed that the modification or the use of $\mathrm{MoS}_{2}$ as a component of a hybrid material does not increase the $\mathrm{q}_{\max }$ towards $\mathrm{Pb}(\mathrm{II})^{399,400}$ and $\mathrm{Hg}(\mathrm{II}),{ }^{395,401,402}$ (see Table 7).

Table 7. Maximum adsorption capacities of TMDs composites used in $\mathrm{Hg}^{2+}$ and $\mathrm{Pb}^{2+}$ metal ions removal process.

\begin{tabular}{c|cccc}
\multicolumn{1}{c}{ Adsorbent } & Contaminant & $\boldsymbol{q}_{\max }\left(\boldsymbol{m g ~ g}^{-1}\right)$ & Conditions & Ref. \\
\hline$W-D R-N-M o S_{2}$ & & $2563^{\mathrm{a}}$ & $\mathrm{pH}=\mathrm{NA}, \mathrm{T}=\mathrm{NA}$ & 395 \\
$\mathrm{Au} / \mathrm{Fe}_{3} \mathrm{O}_{4} / \mathrm{MoS}_{2}$ & $\mathrm{Hg}(\mathrm{II})$ & $1527^{\mathrm{a}}$ & $\mathrm{pH}=5, \mathrm{~T}=293 \mathrm{~K}$ & 402 \\
$\mathrm{MoS}_{2}$ & & $305^{\mathrm{b}}$ & $\mathrm{pH}=6, \mathrm{~T}=308 \mathrm{~K}$ & 401 \\
$\mathrm{MoS}_{2}$ & & $1479^{\mathrm{b}}$ & $\mathrm{pH}=5, \mathrm{~T}=308 \mathrm{~K}$ & 399 \\
$\mathrm{Co}_{\mathrm{MoS}}$ & $\mathrm{Pb}(\mathrm{II})$ & $660^{\mathrm{a}}$ & $\mathrm{pH}=1-6, \mathrm{~T}=293 \mathrm{~K}$ & 400 \\
$M n-M o S_{2}$ & & $588^{\mathrm{a}}$ & $\mathrm{pH}=1-6, \mathrm{~T}=293 \mathrm{~K}$ & 400
\end{tabular}

List of abbreviations. W-DR-N-MoS 2 : widened defect rich nano molybdenum disulfide, Au : gold.

$\mathrm{Q}_{\max }$ values were calculated using (a) Langmuir or (b) Freundlich isotherms.

\subsection{Fluorescence-based metal sensors}


Colorimetric sensors for metal ions comprise two key features, i.e. a metal chelating or binding (coordination) pocket and at least one fluorophore capable of absorbing and/or emitting light. Fluorescence sensing is based on analyte-induced changes in the physicochemical properties of fluorophores including fluorescence intensity, lifetime, and anisotropy, which are related to charge transfer or energy transfer processes. ${ }^{403}$ To function as a sensor, the electronic structure of the sensor must be altered upon metal binding. Changes in the electronic structure of the sensor can lead to the changes in the intensity or wavelength of light absorption or emission, while changes in the molecular structure can modify the distance or alignment between a pair of fluorophores that serve as a donor-acceptor pair.

\subsubsection{Graphene-based fluorescence metal sensors}

Graphene oxide has superb fluorescence quenching capability. ${ }^{404,} 405$ For example, a fluorescent quantum dots (QD)-aptamer-GO sensor based on the nanometal surface energy transfer (NSET) mechanism was designed by Qian et al in order to detect $\mathrm{Pb}^{2+}$ ions. ${ }^{306}$ In particular, photoinduced electron transfer between graphene quantum dots and GO was employed to achieve the controllable fluorescence turn-on process. The capability of QDaptamer-GO nanosensor to detect $\mathrm{Pb}$ (II) and other ions with high sensitivity and good reproducibility was demonstrated on Figure 13.
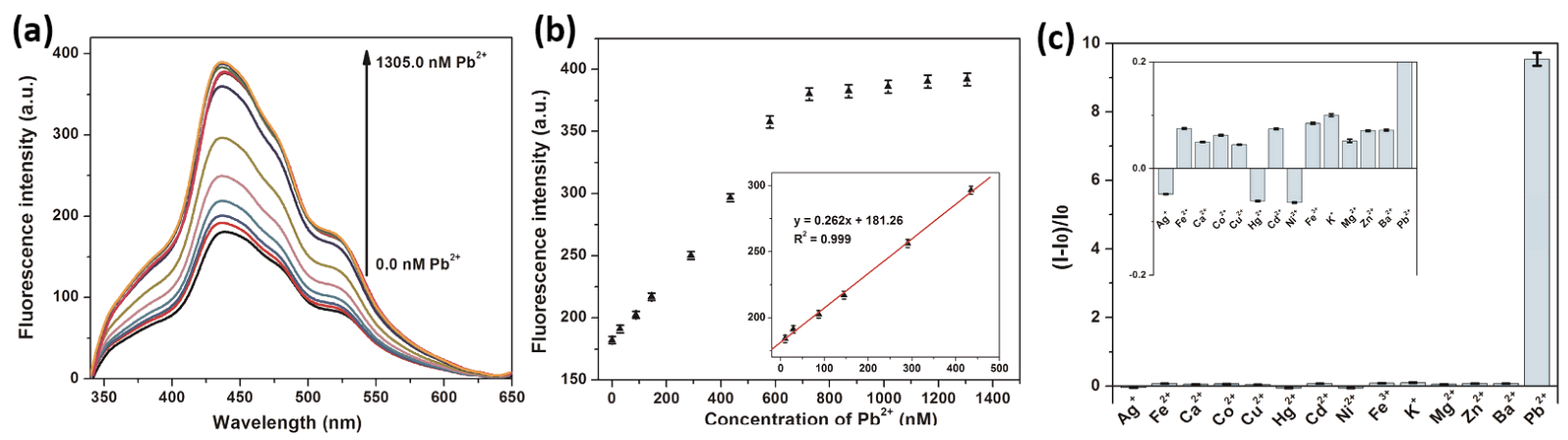

Figure 13. (a) Fluorescence recovery of aptamer-rGQDs/GO system after incubation with various concentrations of $\mathrm{Pb}^{2+}(0.0,29.0,87.0,145.0,290.0,435.0$, 580.0, 725.0, 870.0, 1015.0, 1160.0, $1305.0 \mathrm{nM}$ ). (b) Linear relationship between the fluorescence intensity and concentration of $\mathrm{Pb}^{2+}$. (c) Fluorescence intensity changes $\left(I-I_{0} / I_{0}\right)$ of the sensor in the presence of various metal ions. Adapted from Ref. ${ }^{306}$ with permission of Elsevier Ltd. 
Li et al. demonstrated that the single-stranded DNA (ssDNA) aptamer attached on GO can specifically bind to the mercury ions, leading to the formation of a hairpin-shaped doublestranded DNA (dsDNA) structure. ${ }^{307}$ The water-dispersible GO sheets, which are functionalized with ssDNA aptamer, exhibit strong fluorescence emission at $600 \mathrm{~nm}$ under the excitation of $488 \mathrm{~nm}$ in the absence of $\mathrm{Hg}(\mathrm{II})$ ions. When $\mathrm{Hg}^{2+}$ ions appear in the aqueous solution, they are being sandwiched between the hairpin-shaped dsDNA due to the formation of the thymine- $\mathrm{Hg}(\mathrm{II})-$ thymine complex, which grasps the $\mathrm{Hg}^{2+}$ ions in proximity to the surface of GO. As a result, the fluorescence emission of GO is quenched. Such sensor shows a limit of detection as low as $0.92 \mathrm{nM}$ and excellent selectivity towards $\mathrm{Hg}$ (II) over a wide range of metal ions including $\mathrm{K}^{+}, \mathrm{Ag}^{+}, \mathrm{Ca}^{2+}, \mathrm{Cd}^{2+}, \mathrm{Cu}^{2+}, \mathrm{Pb}^{2+}, \mathrm{Ni}^{2+}, \mathrm{Co}^{2+}$ and $\mathrm{Fe}^{3+}$. Recently, Wen et al. reported a FRET sensor based on a cytosine rich DNA probe and GO. ${ }^{308}$ In the presence of $\mathrm{Ag}^{+}$, a DNA- $\mathrm{Ag}^{+}$complex was formed, and the conformation of the probe changed to straight stiff, resulting in desorption of DNA from the surface of GO and fluorescence recovery (Figure 14a). Fluorescence spectra of cytosine-rich oligonucleotide (SSO) probe upon incubation with a series of concentrations of $\mathrm{Ag}^{+}$and then mixed with GO is portrayed in Figure 14b. This assay is based on the interaction between the target-induced conformational change of the SSO fluorogenic probe and graphene oxide quenching effects. The use of a simple mix-and-detect analysis revealed a high selectivity toward $\mathrm{Ag}^{+}$as determined in the presence of a ten times higher concentration of 12 different interference metal ions (Figure 14c).
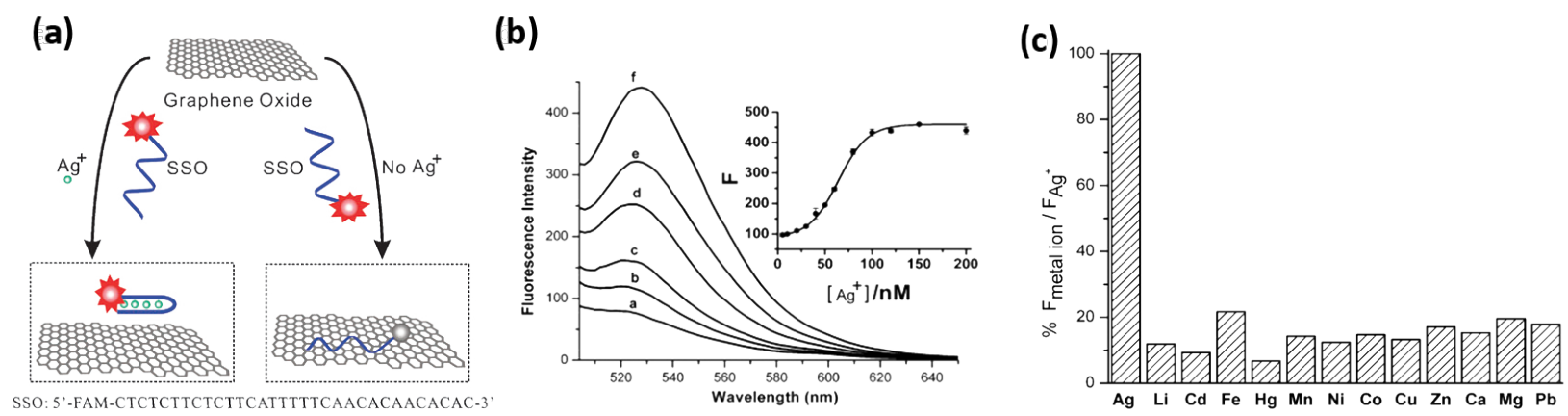

Figure 14. Schematic illustration of the fluorescence sensor for $\operatorname{Ag}(I)$ ions based on the target-induced conformational change of a silver specific cytosine-rich oligonucleotide (SSO) and the interactions between the fluorogenic SSO probe and graphene oxide. SSO: (a) FAMlabelled silver-specific oligonucleotide probe. (b) Fluorescence spectra of SSO probe upon incubation with a series of concentrations of $\mathrm{Ag}^{+}$and then mixed with GO: $a 0 \mathrm{M}, b 20 \mathrm{nM}, c$ 
$40 \mathrm{nM}, d 60 \mathrm{nM}, e 80 \mathrm{nM}$ and $f 150 \mathrm{nM}$. All experiments were carried out in MOPS buffer (10 mM, pH 7.0) containing $50 \mathrm{mM}$ of $\mathrm{NaNO}_{3}$ and $10 \mathrm{nM}$ of SSO. $\mathrm{Ag}^{+}$of different concentrations were incubated in this solution for $5 \mathrm{~min}$ at $23^{\circ} \mathrm{C}$ and then the fluorescence spectra were recorded $2 \mathrm{~min}$ after GO $(10 \mathrm{mg})$ were added to this mixture. $\lambda_{\mathrm{ex}}=494 \mathrm{~nm}$. (c) Selectivity of the analysis of $\mathrm{Ag}^{+}$ions in the presence of different metal ions. The concentration of $\mathrm{Ag}^{+}$was $100 \mathrm{nM}$ whereas all other interference metal ions were $1 \mu \mathrm{M}$. Adapted from Ref. ${ }^{308}$ with permission of the Royal Society of Chemistry.

\subsubsection{Transition metal dichalcogenides based fluorescence metal sensors}

As a result of their finite band gap, TMDs exhibit interesting optical properties. By mastering the selective recognition capabilities of TMDs toward specific heavy metal ions, several fluorescence sensors for metal ions detection have been developed recently, highlighting the promise of using TMDs-based materials to construct novel nanoprobes for chemical sensing and in particular for detection of metal ions. ${ }^{309-314}$ For heavy metal ion sensors, TMDs are either non-covalently functionalized with molecules, which are used as sensing probes - because of their high binding affinity to heavy metal ions, ${ }^{314}$ or covalently modified, e.g. through N- or B- doping. ${ }^{311}$ Mao et al. reported a seminal work on the use of a single layer $\mathrm{MoS}_{2}$ as the fluorescence quencher to design a probe for detection $\mathrm{Ag}^{\mathrm{I}}$ with excellent sensitivity and selectivity. The detection limit in this assay was found being as low as $1 \mathrm{nM}$ for $\mathrm{Ag}^{+} .309$

Recently, a fluorescent nanoprobe based on $\mathrm{MoS}_{2}$ nanosheets for selective and ultrasensitive detection of $\mathrm{Ag}^{+}$(down to $10 \mathrm{nM}$ ) ions alike in aqueous solutions and biological cells (Escherichia coli) has been developed by Yang et al. ${ }^{310}$ In this study $\mathrm{Ag}^{\mathrm{I}}$ ions were reduced to $\mathrm{Ag}^{0}$, which led to the detachment of Rhodamine B isothiocyanate (RhoBS) non-covalently interacting with $\mathrm{MoS}_{2}$ nanosheets, which resulted in quenching of its fluorescence (see Table 8 for details).

An interesting approach for enhancing the fluorescence of $\mathrm{MoS}_{2}$ has been proposed by Liu et. $a l .{ }^{311}$ and relies on covalent modification of $\mathrm{MoS}_{2}$ nanosheets with either boron or nitrogen atoms. Boron and nitrogen doping results in changes of the band gap of $\mathrm{MoS}_{2}$ which increases from $1.20 \mathrm{eV}$ to $1.61 \mathrm{eV}$. The as-prepared $\mathrm{B}$ - and $\mathrm{N}$-doped $\mathrm{MoS}_{2}$ nanosheets were used as facile, green, label-free and effective sensing platforms for $\mathrm{Hg}^{2+}$ ions. The modified 
nanosheets exhibit enhanced fluorescence properties compared with the undoped $\mathrm{MoS}_{2}$, which were highly quenched after selective absorption of $\mathrm{Hg}^{2+}$. Wang et. al. found out that after the doping with $\mathrm{Pb}^{2+}$ ions, the fluorescence of $\mathrm{MoS}_{2}$ nanosheets was enhanced, which was subsequently quenched by the addition of sulphide ions. ${ }^{312}$ The fluorescence quantum yield measurements of the single layer $\mathrm{MoS}_{2}$ was estimated being ca. $0.28 \%$. The quantum yield of the doped $\mathrm{MoS}_{2}$ nanosheets was calculated to be $c a .0 .73 \%$, thereby indicating that molecular doping of $\mathrm{MoS}_{2}$ nanosheets with lead(II) metal ions strengthened their fluorescence.

To date little has been done on the application of $\mathrm{WS}_{2}$ nanosheets in biological and chemical sensing. For example, Zuo et al. developed a novel dual-colour fluorescent biosensing platform based on $\mathrm{WS}_{2}$ nanosheets. ${ }^{313}$ The sensor could achieve simultaneous detection of $\mathrm{Hg}^{2+}$ and $\mathrm{Ag}^{+}$in a high sensitivity and wide linear range by monitoring fluorescence intensity changes at $525 \mathrm{~nm}$ and $583 \mathrm{~nm}$, respectively. $\mathrm{Hg}^{2+}$ and $\mathrm{Ag}^{+}$were selectively detected in the concentration range from 6.0 to $650.0 \mathrm{nM}$ and from 5.0 to $1000.0 \mathrm{nM}$, with detection limits of $3.3 \mathrm{nM}$ and $1.2 \mathrm{nM}$, respectively. The fluorescence intensities in the presence of other heavy metal ions changed by ca. $5 \%$ when cations concentrations were 10 -fold greater than that of $\mathrm{Hg}^{2+}$ and $\mathrm{Ag}^{+}$ions, indicating that the developed biosensor is highly selective for mercury and lead ions.

\subsubsection{Other 2DMs based fluorescence metal sensors}

Liu et al. showed an integrated black phosphorus-tilted fibre grating (TFG) configuration as an enhanced light-matter interaction platform for heavy metal sensing. ${ }^{315}$ BP nanosheets were produced by liquid-phase exfoliation and deposited through in situ layer-by-layer technique. The BP-TFG composite was exploited as the optic probe for $\mathrm{Pb}^{2+}$ ions detection, and the results showed ultrahigh sensitivity up to $0.5 \times 10^{-3} \mathrm{~dB} / \mathrm{ppb}$, limit of detection down to 0.25 $\mathrm{ppb}$, and extremely wide concentration range from $0.1 \mathrm{ppb}$ to $1.5 \times 10^{7} \mathrm{ppb}$. The achievable limit of detection was estimated as 40 times lower than World health organization permissible limit for lead in drinking water. The wide detection range is 4-orders of magnitude larger than that of BP-FET based lead sensor. ${ }^{316}$

$\mathrm{Gu}$ et al. presented novel ratiometric fluorescence sensor based on the inner filter effect (IFE) of tetraphenylporphyrin tetrasulfonic acid (TPPS) toward black phosphorus quantum dots 
(BP QDs), developed for the selective and sensitive detection of $\mathrm{Hg}^{2+} \cdot 317$ Highly fluorescent BP QDs were successfully synthesized from bulk BP by sonication-assisted solvothermal method. In the presence of $\mathrm{Hg}^{2+}$, the IFE originating from the spectral overlap between the excitation of BP QDs and the absorption of TPPS is inhibited; yielding in the recovery of the fluorescence of BP QDs. The constructed sensor revealed a good linear response to $\mathrm{Hg}^{2+}$ ranging from 1 to $60 \mathrm{nM}$ with a detection limit of $0.39 \mathrm{nM}$. Interestingly, this strategy could be also applied in the determination of $\mathrm{Hg}^{2+}$ in real water samples with satisfactory results. Sensing properties for all 2DMs-based fluorescent sensors are presented in Table 8.

Table 8. Comparison of sensing properties of different TMDs based fluorescent sensor.

\begin{tabular}{c|cccc}
\multicolumn{1}{c}{ Material } & Analyte & Linear range & Detection limit & Ref. \\
\hline$M_{2}$ & $\mathrm{Ag}(\mathrm{I})$ & $10-500 \mathrm{nM}$ & $1 \mathrm{nM}$ & 309 \\
$W S_{2}$ & & $5.0-1000 \mathrm{nM}$ & $1.2 \mathrm{nM}$ & 313 \\
$B, N-M o S_{2}$ & & $6.0-650 \mathrm{nM}$ & $3.3 \mathrm{nM}$ & 313 \\
$B P-T P P S$ & $\mathrm{Hg}(\mathrm{II})$ & $0.01-3 \mu \mathrm{M}$ & $1 \mathrm{nM}$ & 311 \\
$M o S_{2}$ & & $1-60 \mathrm{nM}$ & $0.39 \mathrm{nM}$ & 317 \\
$B P-f i b r e s$ & & $0.5-12 \mu \mathrm{M}$ & $0.22 \mu \mathrm{M}$ & 312 \\
\end{tabular}

List of abbreviations. TPPS : tetraphenylporphyrin tetrasulfonic acid.

\subsection{Field-Effect Transistor based metal sensor}

2DMs nanosheets integrated in FET have recently revealed their enormous potential for detection of heavy metals. The working principle of 2DM-based FET sensor is based on the changes of the critical parameters of a FET containing 2DMs nanosheets upon adsorption of targeted heavy metal ions. This includes primarily the field-effect mobility, threshold voltage and $\mathrm{I}_{\mathrm{on}} / \mathrm{I}_{\mathrm{off}}$ ratio. 2D semiconducting sheets are of particular interest because their high charge carrier mobility and very high surface-to-volume ratio, leading to high sensitivity. Chemical sensors based on FETs can overcome the obstacles of previous detection methods. For example, aforementioned optical methods have some limitations such as multiple sensing steps, the need for using chemical agents, a higher cost, and a longer detection time. In contrast, the use of 2DM-based FET sensors enables the rapid label-free detection of metal ions in real-time by monitoring the resistance or the Dirac point shift caused by the 
adsorption of target analytes. Such devices can be characterized also by low power consumption and can be miniaturized for the development of portable sensors, eventually supported on flexible foils.

\subsubsection{Graphene-based FET metal sensors}

In a typical graphene field-effect transistor (GFET) based sensor, graphene is used as conducting material in the channel between drain and source electrodes. Gate potential is applied through back-gate (typical thin $\mathrm{SiO}_{2}$ layer) ${ }^{406}$ or top-gate (electric double layer in electrolyte). ${ }^{114}$ The absorption of analyte molecules or change of local environment leads to the change of graphene electrical conductance. Zhang et al. reported the functionalization of mechanically exfoliated graphene with a self-assembled monolayer of 1-octadecanethiol and its application in $\mathrm{Hg}^{2+}$ sensing. ${ }^{290}$ Substituted alkane derivatives were found to self-assemble into large-scale highly-ordered physisorbed monolayers on single-layer graphene supported by the $\mathrm{SiO}_{2}$ dielectric substrate. According to AFM imaging, the height of graphene increased to $1.6 \mathrm{~nm}$ when exposed to $\mathrm{Hg}^{2+}$, indicating the successful uptake of mercury ions by the thiol groups exposed on the graphene surface. Such graphene-FET sensor revealed a detection limit for $\mathrm{Hg}^{2+}$ as low as $10 \mathrm{ppm}$.

Alternative approaches based on solution processable rGO, ${ }^{291,}{ }^{292}$ and G functionalization ${ }^{92}$, 293-295 are being pursued with the ultimate goal of developing low-cost, scalable fabrication of graphene-FET sensors. Recently, Sudibya et al. presented a FET sensor using micropatterned, metallothionein type II protein (MT II)-functionalized rGO films which bind with both physiological (e.g., $\mathrm{Cu}^{2+}, \mathrm{Zn}^{2+}$ ) and xenobiotic (e.g., $\mathrm{Hg}^{2+}, \mathrm{Cd}^{2+}$ ) metals ions with high affinity (see schematic illustration in Figure 15a). ${ }^{291}$ A typical plot of drain-to-source current $\left(\mathrm{I}_{\mathrm{ds}}\right)$ versus solution-gate voltage $\left(\mathrm{V}_{\mathrm{g}}\right)$ of rGO-FET sensor is displayed in Figure 15b. Such a nanoelectronic sensor is capable of detecting various metal ions in real-time with high sensitivity. The addition of mercury $\left(\mathrm{Hg}^{2+}\right)$, at a concentration as low as $1 \mathrm{nM}$, caused the obvious current increase in the rGO-FET which was biased at $\mathrm{V}_{\mathrm{ds}}=400 \mathrm{mV}$ and $\mathrm{V}_{\mathrm{g}}=-0.6 \mathrm{~V}$ (Figure 15c). The magnitude of the device response scales with the $\mathrm{Hg}^{2+}$ concentration, and its polarity depends on the gate voltage $\left(\mathrm{V}_{\mathrm{g}}\right)$. The detection limit for $\mathrm{Hg}^{2+}$ was estimated being as low as $\sim 1 \mathrm{nM}$ with a signal-to-noise ratio of 25-30 (Figure 15d). The very same device exhibited also $\mathrm{Cd}^{2+}$ detection at $1 \mathrm{nM}$ with a slightly smaller change of current signalto-noise ratio of 15-20 (Figure 15e). 

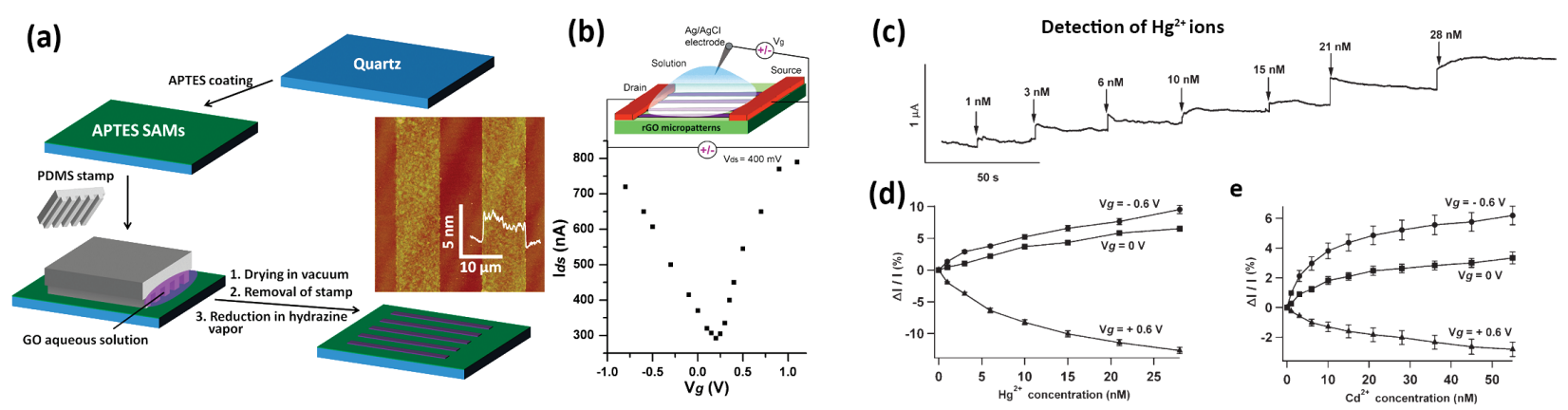

Figure 15: (a) Schematic illustration for fabrication of patterned rGO thin films on APTEScoated quartz. Inset: AFM image of the obtained rGO micropatterns on APTES-coated quartz. (b) Ambipolar characteristics of rGO-FET measured in $0.1 \mathrm{M}$ of phosphate buffer saline (PBS) solution. Inset: Schematic of solution-gated configuration of rGO-FET. (c) Typical real-time recording of $\mathrm{I}_{\mathrm{ds}}$ with the addition of $\mathrm{Hg}^{2+}$ ions. (d,e) Change of $\mathrm{I}_{\mathrm{ds}}$ in $\mathrm{rGO}$ FETs (tested sample number $\mathrm{n}=6$ ) with the addition of $(\mathrm{d}) \mathrm{Hg}^{2+}$ and (e) $\mathrm{Cd}^{2+}$ ions at $\mathrm{V}_{\mathrm{ds}}=0.4$ $\mathrm{V}$ and $\mathrm{V}_{\mathrm{g}}=-0.6$ (circle), 0 (square), and $+0.6 \mathrm{~V}$ (triangle). Adapted from Ref. ${ }^{291}$ with permission of American Chemical Society.

For heavy metal ion GFET sensors, biomolecules are frequently used as sensing nanoprobes due to their high binding affinity to inorganic contaminants. ${ }^{92}$ An et. al., reported highperformance flexible graphene aptasensor for $\mathrm{Hg}^{2+}$ detection. ${ }^{293}$ 1,5-diaminonaphthalene (DAN) and glutaraldehyde (GA) were employed as cross-linking agents, while the aptamer (3'-amine-TTC TTT CTT CCC CTT GTT TGT-C10 carboxylic acid-5') was non-covalently linked onto the graphene surface as a probe for $\mathrm{Hg}^{2+}$. The field-induced responses from the graphene aptasensor had excellent sensing performance: $\mathrm{Hg}^{2+}$ ions with very low concentration of $10 \mathrm{pM}$ could be detected (Figure 16a) being 2-3 orders of magnitude higher than previously reported mercury sensors using electrochemical devices. ${ }^{406,407}$ GFET-sensor was characterized with the experimental setup presented schematically in Figure 16b. The sensor response time was rapid, with values below $1 \mathrm{~s}$. Noteworthy, the pristine graphene devices showed no significant current changes upon the exposure to $\mathrm{Hg}^{2+}$. It was concluded, that the origin of current changes in the sensor relies on the $p$-doping effect resulting from the thymine- $\mathrm{Hg}$-thymine complex formed between $\mathrm{Hg}^{2+}$ ions and thymine base pairs in the aptamer (Figure 16c). 

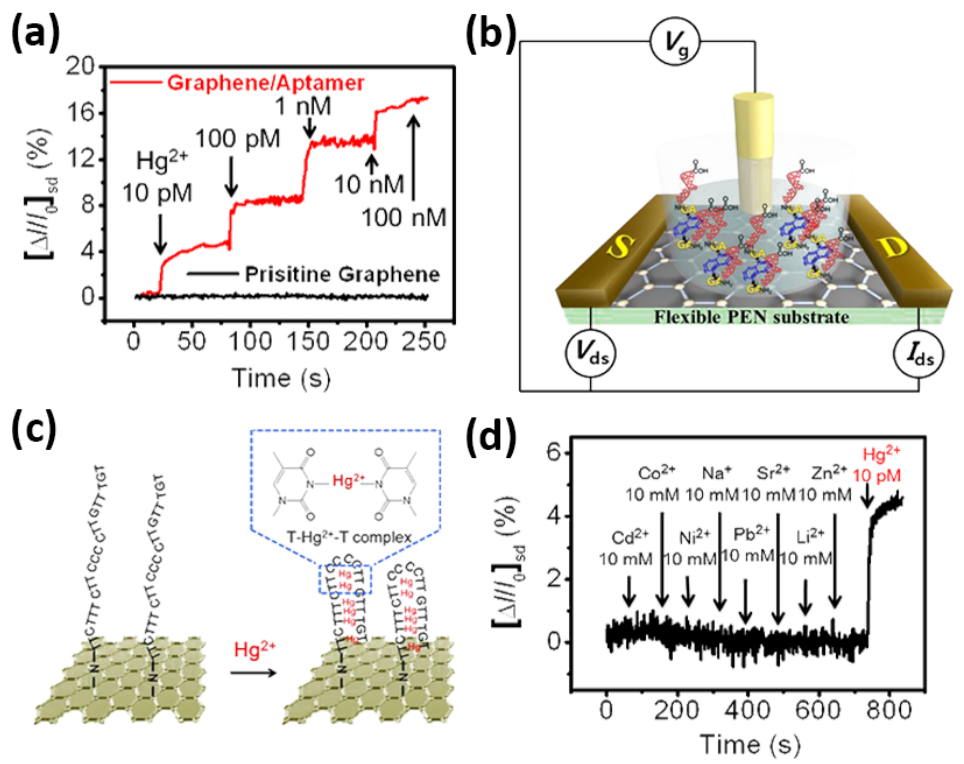

Figure 16. (a) Real-time responses curve of the aptasensor with various $\mathrm{Hg}^{2+}$ concentrations $(10 \mathrm{pM}$ to $100 \mathrm{nM})$. Graphene substrate without aptamer was introduced as a control sample.

(b) Schematic diagram of a liquid-ion gated FET using graphene conjugated with aptamer. $\left(\mathrm{V}_{\mathrm{g}}, \mathrm{S}\right.$ and $\mathrm{D}$ indicate gating voltage and source/drain electrodes). (c) Interaction of $\mathrm{Hg}^{2+}$ ions with thymine base pairs in the aptamer immobilized on the surface of the modified graphene layer (d) Selective responses of the aptasensor toward target metal ion $\left(\mathrm{Hg}^{2+}, 10 \mathrm{pM}\right)$ and nontarget metal ions $\left(\mathrm{Cd}^{2+}, \mathrm{Co}^{2+}, \mathrm{Ni}^{2+}, \mathrm{Na}^{+}, \mathrm{Pb}^{2+}, \mathrm{Sr}^{2+}\right.$ and $\left.\mathrm{Zn}^{2+}, 10 \mathrm{mM}\right)$. Adapted from Ref. ${ }^{293}$ with permission of American Chemical Society.

Noteworthy, graphene-based FET sensors can be exploited not only for the detection heavy metal ions (e.g. $\mathrm{Hg}^{2+}$ and $\mathrm{Pb}^{2+}$ ) but also alkali metal ions in water including $\mathrm{K}^{+294}$ and $\mathrm{Na}^{+}{ }^{295}$

\subsubsection{Transition metal dichalcogenides FET metal sensors}

The semiconducting properties of $\mathrm{MoS}_{2}$ make this $2 \mathrm{DM}$ particularly suitable as electroactive material for applications in transistor. ${ }^{408}$ Also because of this reason, $\mathrm{MoS}_{2}$ has been employed in FET sensors for detecting heavy metal ions. ${ }^{296,297}$

Jiang et al. demonstrated the FET based on mechanically exfoliated few-layer $\mathrm{MoS}_{2}$ nanosheets for sensing $\mathrm{Hg}^{2+}$ ions. ${ }^{296}$ The interaction between $\mathrm{Hg}^{2+}$ ions and few-layer $\mathrm{MoS}_{2}$ were studied by FET measurements and photoluminescence. Due to a high binding affinity 
between the sulphur sites on the surface of $\mathrm{MoS}_{2}$ surface and $\mathrm{Hg}^{2+}$ ions, the latter can strongly bind to $\mathrm{MoS}_{2}$. Remarkably, it was shown that the binding of $\mathrm{Hg}^{2+}$ results in a p-type doping and reduces the electrons concentration in $n$-type few-layer $\mathrm{MoS}_{2}$. Upon binding of $\mathrm{Hg}^{2+}$ ions the electron transport and photoluminescence properties in few-layer $\mathrm{MoS}_{2}$ are effectively modulated. It was also demonstrated, that by monitoring the changes in conductance of few-layer $\mathrm{MoS}_{2}$ and varying the concentration of $\mathrm{Hg}^{2+}$ solutions, few-layer $\mathrm{MoS}_{2}$ transistors can function as highly sensitive sensors for rapid electrical detection of $\mathrm{Hg}^{2+}$ with a detection limit as low as $30 \mathrm{pM}$.

Zhou et al. reported on a DNA-functionalized $\mathrm{MoS}_{2}$ nanosheet/gold nanoparticle hybrid FET sensor for the ultrasensitive detection of $\mathrm{Hg}^{2+}$ in an aqueous environment (Figure 17a). ${ }^{297} \mathrm{~A}$ thin film was formed by filtration of $\mathrm{MoS}_{2}$ nanosheets produced by liquid-phase exfoliation, followed by transfer onto the Au electrodes and thermal annealing. Then Au NPs were sputtered onto the $\mathrm{MoS}_{2}$ film, and finally the DNA molecules were grafted onto Au NPs by immersing the device in a DNA solution. In the hybrid structure, the $\mathrm{MoS}_{2}$ thin film acts as the conducting channel with the homogeneously dispersed Au NPs operating as anchoring sites for DNA probes chosen to specifically target $\mathrm{Hg}^{2+}$ ions. Upon addition of $\mathrm{Hg}^{2+}$ to the sensor, the formation of metal complexes between the thymidine bases present in the DNA molecules and mercury ions takes place, resulting in the changes of the $\mathrm{MoS}_{2}$ conductance. The detection of metal ions was enabled by monitoring the change of the source-drain current in the FET device as a function of $\mathrm{Hg}^{2+}$ concentration. The detection limit of the sensor can reach values down to the concentration of $0.1 \mathrm{nM}$ (Figure 17b). It was shown that the rate of the increase in the conductance or source-drain current is dependent on the $\mathrm{Hg}^{2+}$ concentration (Figure 17c). The sensor was also tested with a series addition of $\mathrm{Hg}^{2+}$ and the dynamic responses indicated that the sensor responded to $\mathrm{Hg}^{2+}$ within a few seconds, which is much faster than the conventional optical methods. The output characteristics of three types of sensors, i.e. $\mathrm{MoS}_{2}, \mathrm{MoS}_{2}-\mathrm{Au}$ NPs, $\mathrm{MoS}_{2} / \mathrm{DNA}-\mathrm{Au}$ NPs are shown in Figure 17d. Because of the work function difference between the $\mathrm{Au} N P s(3.6 \mathrm{eV}$ in air for $\mathrm{D}=8 \mathrm{~nm})$ and the $\mathrm{MoS}_{2}$ film ( $\sim 5.23 \mathrm{eV}$ after annealing), electron transfer occurs from the Au NPs to the $\mathrm{MoS}_{2}$ film, leading to a decreased concentration of holes in the $\mathrm{MoS}_{2}$ film and thus the decreased conductivity. 
(a)

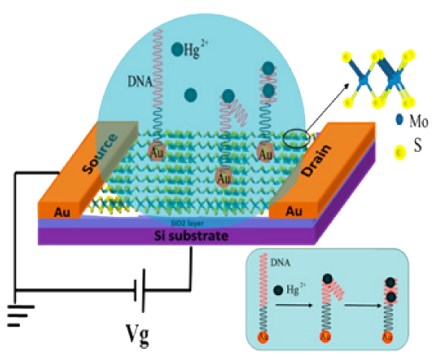

(c)

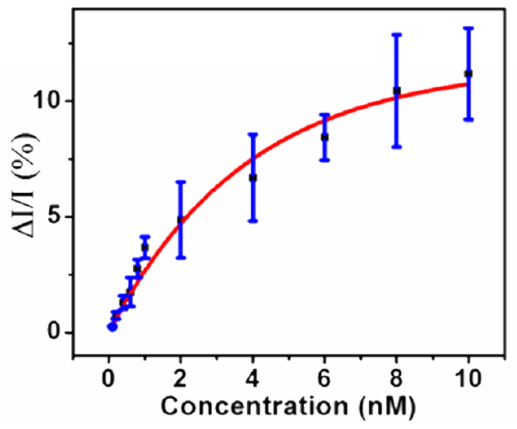

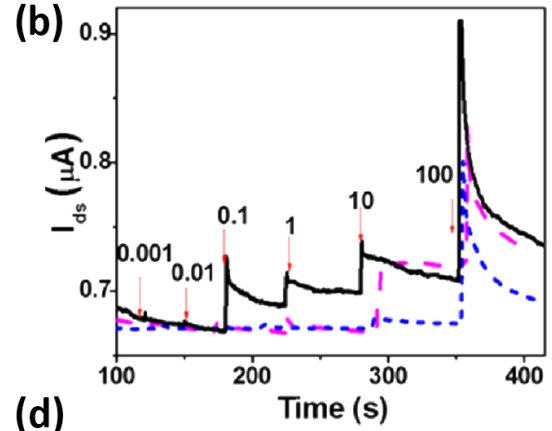

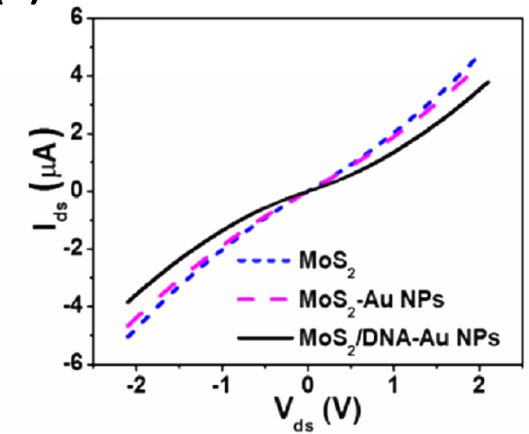

Figure 17. (a) FET sensor platform based on the hybrid structure. The formation of $\mathrm{T}-\left(\mathrm{Hg}^{2+}\right)$ $\mathrm{T}$ chelates, through reaction between $\mathrm{Hg}^{2+}$ and the thymidine bases exposed on the DNA molecules grafted to the Au NPs, leading to the change in the $\mathrm{MoS}_{2}$ electrical conductivity as a sensor signal. (b) Real-time detections of $\mathrm{Hg}^{2+}(\mathrm{nM})$ in water $\left(\mathrm{V}_{\mathrm{ds}}=0.1 \mathrm{~V}\right)$ with platforms of $\mathrm{MoS}_{2}$ /DNA-Au NPs (black, solid), $\mathrm{MoS}_{2}-\mathrm{Au}$ NPs (purple, dash), $\mathrm{MoS}_{2}$ (blue, short dash), respectively. (c) Sensitivity variation and exponential fitting of sensitivity as a function of $\mathrm{Hg}^{2+}$ concentration for the $\mathrm{MoS}_{2} / \mathrm{DNA}-\mathrm{Au}$ NPs hybrid sensor. (d) Evolution of the $\mathrm{I}_{\mathrm{ds}}-\mathrm{V}_{\mathrm{ds}}$ characteristics during the $\mathrm{MoS}_{2} / \mathrm{DNA}-\mathrm{Au}$ NPs hybrid sensor fabrication process $\left(\mathrm{V}_{\mathrm{ds}}=-2.1 \mathrm{~V}\right.$ to $2.1 \mathrm{~V}$, step $=0.1 \mathrm{~V}$ ) at room temperature. Adapted from Ref. ${ }^{297}$ with permission of American Chemical Society.

Despite the examples discussed in this Review, little knowledge has been gathered on the sorption capacity of other metal ions on $2 \mathrm{D} \mathrm{MoS}$. Therefore, it is of significant importance to extend in the future the application of such $2 \mathrm{DM}$ for the detection or removal of other heavy metals considering its markedly high metal capture capacity. 


\subsubsection{Other 2DMs based FET metal sensors}

Li et al as the first presented ultra-sensitive suspended black phosphorus FET sensor for detection mercury ions operated in sub-threshold regime. ${ }^{298}$ Thin BP nanosheets were mechanically exfoliated by scotch tape based method and transferred onto low resistance $\mathrm{Si}$ substrate covered with a $300 \mathrm{~nm}$ thick layer of $\mathrm{SiO}_{2}$. BP FET is a transducer, which converts the adsorption of ions into conductivity/current shift by gating effect. Free-standing BP FET sensors gated into sub-threshold regime demonstrated ultrahigh sensitivity which was almost two orders of magnitude larger than that of non-suspended BP in linear regime. The proposed BP sensors were able to realize rapid ( $3 \mathrm{~s}$ ) label-free detection of $\mathrm{Hg}^{2+}$ down to $0.01 \mathrm{ppb}$, and superb selectivity was achieved by functionalizing BP surface with mercury ionophore. BP sensors tested with different thicknesses revealed that the response $\left(\Delta \mathrm{R} / \mathrm{R}_{0}\right)$ to $10 \mathrm{ppb} \mathrm{Hg}^{2+}$ decreased approximately 30\% when BP channel thickness varied from $8 \mathrm{~nm}$ to $20 \mathrm{~nm}$

The same group demonstrated also air stable high-performance BP chemical sensors encapsulated with ionophore (Figure 18a, inset). ${ }^{316}$ Without protection, BP samples once exposed to air start to degrade and oxygenated phosphorus $\left(\mathrm{PO}_{\mathrm{X}}\right)$ is shortly formed, with degradation of its electric properties and device performances. Ionophore film effectively reduces negative factors from ambient environment; meanwhile, it displays selective permeability towards certain type of molecules. The ionophore-encapsulated BP devices were found being still in good shape after 1 week of ambient exposure, with source-drain current $\mathrm{I}_{\mathrm{DS}}$ variation less than $10 \%$ (Figure 18a). The methodology of preparation thin BP nanosheets is the same as the one shown above (i.e. mechanical exfoliation with scotch tape). The BP sensors were sensitive to the detection of multiplex ions such as $\mathrm{AsO}^{2-}, \mathrm{Hg}^{2+}, \mathrm{Cd}^{2+}$, and $\mathrm{Pb}^{2+}$ with detection limit down to $10,1,3$ and $1 \mathrm{ppb}$, respectively. Moreover, $\mathrm{Pb}^{2+}$ ions can be effectively detected over a wide concentration range, from $10 \mathrm{ppb}$ to $100,000 \mathrm{ppb}$ (Figure 18b). The detection limit and response rate of BP are both better than those reported for graphene based sensors. 
(a)

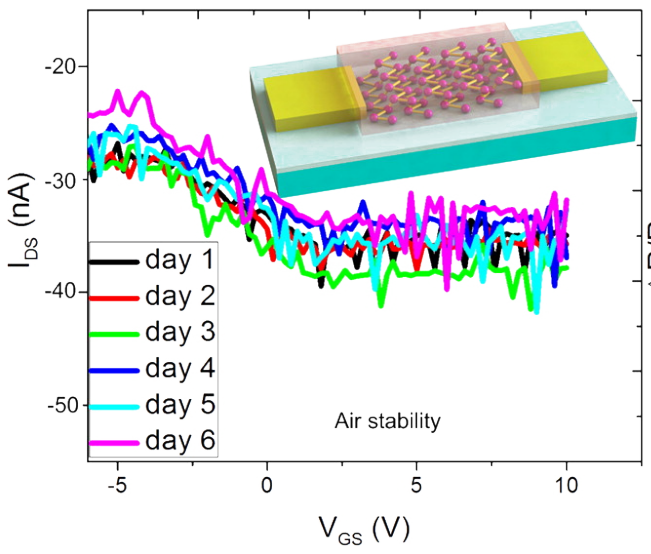

(b)

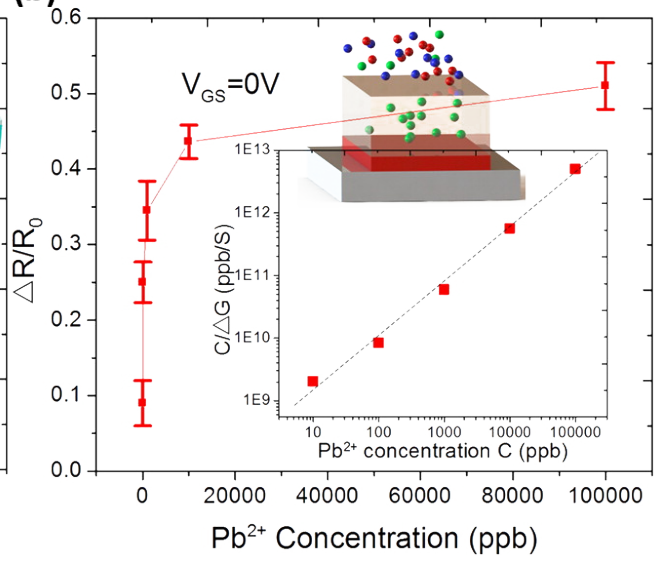

Figure 18. (a) Schematic view of a BP sensor with $I_{D S}$ vs $V_{G S}$ curves of $B P$ with ionophore protection. $\mathrm{I}_{\mathrm{DS}}$ variation is less than $10 \%$, suggesting significantly improved air stability. (b) $\Delta \mathrm{R} / \mathrm{R}_{0}$ versus $\mathrm{Pb}^{2+}$ concentration (experimental results from 7 sensors) and schematic view of BP sensors with lead ionophore. Resistance of BP shows saturated at higher concentrations. Experimental results demonstrate good linear relation between $(\mathrm{C} / \Delta \mathrm{G})$ and $\mathrm{C}$, fitting well the Langmuir adsorption isotherm, as shown in the inset. Adapted from Ref. ${ }^{316}$ with permission of American Chemical Society.

Although only a few biosensors and water sensors with BP FETs were reported due to the inherent instability of $\mathrm{BP}$ in a moisture rich environment, BP sensors have attracted increasing interest across the sensing community due to the 2D structure, tuneable band gap, and high carrier mobility of BP. From a practical application standpoint, the thickness, uniform control, and stability of BP are limiting its application in ultrasensitive sensor technology. With further understanding of interactions between BP and oxygen/water and the improvement of sensor fabrication techniques, BP-based FET sensors can be foreseen to provide a robust sensing method for a wide variety of target analytes in the near future.

\subsection{Electrochemical based metal sensors}

Electrochemical sensing of heavy metal ions relies on the use of sensing electrodes that are employed for passing the current to the aqueous solution and generate electrical signal that corresponds to the electrochemical reaction within the solution due to presence of metal ions. Common experimental setups for electrochemical detection of heavy metal ions consist of an 
electrolytic cell containing an electrolyte, i.e. solution of heavy metal ions, in contact with an electrode. The cell potential is measured at the interface of the electrode with the electrolyte solution. Noteworthy, because heavy metal ions have defined redox potential, the selectivity toward specific heavy metal ions can be achieved by using bare electrodes without the need of a molecular recognition probe. Numerous techniques are employed in electrochemical sensing, including potentiometry, voltammetry, impedimetry, amperometry and conductometry. In particular, the anodic stripping voltammetry (ASV) method is widely explored for detection of heavy metals. ASV analysis typically involves two steps, i.e. deposition of heavy metals onto the electrode surface, and stripping or dissolution of the deposited analyte from the electrode surface. Recent advancements in the field have revealed the potential of 2DMs in electroanalysis. Several electrochemical sensors based on 2DMs for bioanalysis and environmental analyses have been developed. In particular, Zhao et al. presented for the first time that $\mathrm{Hg}^{2+}$ can be selectively identified using a PPy-rGO nanocomposite-modified glassy carbon electrode (GCE). ${ }^{299}$ Such selectivity was achieved by using square wave anodic stripping voltammetry (SWASV), which determines a reduction of adsorbed $\mathrm{Hg}^{2+}$ to $\mathrm{Hg}^{0}$ at a certain potential. The anodic stripping current was obtained in a potential range for the identification of $\mathrm{Hg}^{2+}$. In addition, excellent sensitivity $\left(0.124 \mu \mathrm{A} \mathrm{nM}^{-}\right.$ $\left.{ }^{1}\right)$ and limit of detection (LOD) (15 nM) results were achieved. The measured stripping current toward $\mathrm{Hg}^{2+}$ at the PPy modified electrode was found to be 3-9 times higher than that towards other ions, indicating that $\mathrm{rGO}$ in the nanocomposite plays an important role in the highly selective detection.

Sahhoo et al. reported a facile in situ approach for the fabrication rGO/bismuth(Bi) nanocomposite by employing modified Hummers method without the use of any surfactants. Bi nanoparticles were uniformly anchored onto the surfaces of individual graphene nanosheets, which prevent restacking of $\mathrm{rGO}$, resulting in good dispersion in solvents. $\mathrm{rGO} / \mathrm{Bi}$ nanocomposite was used as an electrode material for the stripping voltammetric determination of heavy metal ions in water. The detection limits of the proposed electrochemical sensor for $\mathrm{Cd}^{2+}, \mathrm{Pb}^{2+}, \mathrm{Zn}^{2+}$ and $\mathrm{Cu}^{2+}$ were found amounting to 2.8, 0.55, 17 and $26 \mathrm{ppb}$, respectively. ${ }^{300}$ Wei et al. developed a $\mathrm{SnO}_{2} / \mathrm{rGO}$-based electrochemical sensor, which could simultaneously and selectively analyse four heavy metal ions such as $\mathrm{Cd}(\mathrm{II})$, $\mathrm{Pb}(\mathrm{II}), \mathrm{Cu}(\mathrm{II})$ and $\mathrm{Hg}(\mathrm{II}) .{ }^{301} \mathrm{SWASV}$ has been employed for the detection of heavy metal ions (Figure 19a). The $\mathrm{SnO}_{2} / \mathrm{rGO}$ nanocomposite modified glass carbon electrode synthesized 
by a simple wet chemical method showed enhanced sensing performance compared with single $\mathrm{SnO}_{2}$ and single rGO (Figure 19b).
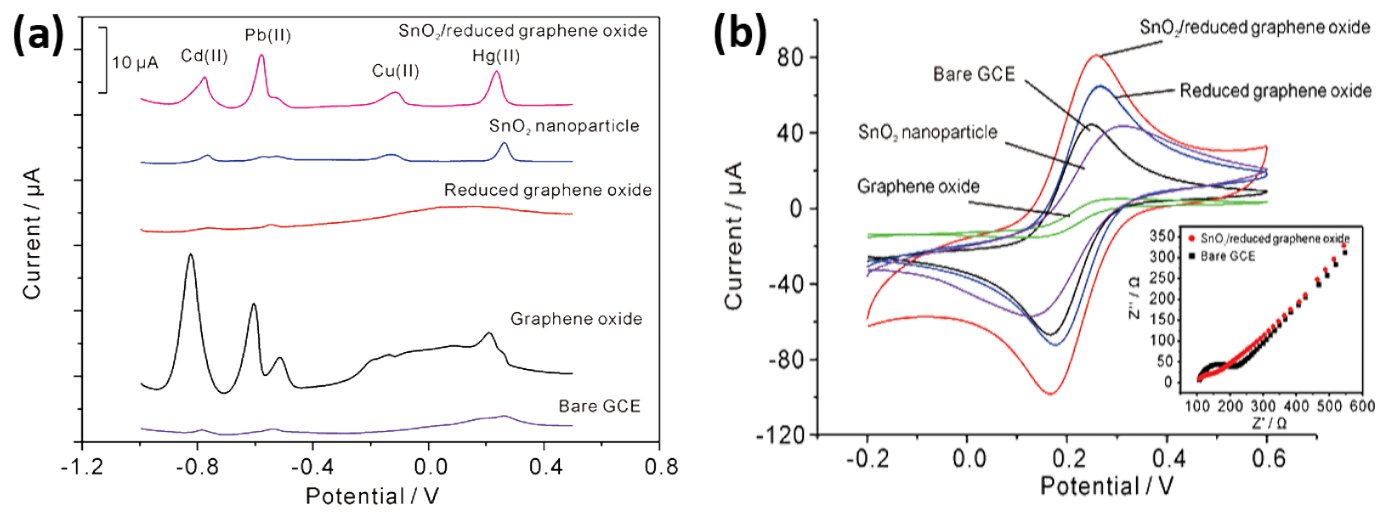

Figure 19. (a) Square Wave Anodic Stripping Voltammetry (SWASV) curves for $0.5 \mu \mathrm{M}$ each of $\mathrm{Cd}(\mathrm{II}), \mathrm{Pb}(\mathrm{II}), \mathrm{Cu}(\mathrm{II})$, and $\mathrm{Hg}(\mathrm{II})$ on bare (violet line), graphene oxide (black line), reduced graphene oxide (red line), $\mathrm{SnO}_{2}$ nanoparticle (blue line), and $\mathrm{SnO}_{2} /$ reduced graphene oxide nanocomposite (pink line) modified GCE in 0.1 M acetate buffer ( $\mathrm{pH}$ 5.0). Deposition potential $=-1.0 \mathrm{~V}$; deposition time $=120 \mathrm{~s}$; amplitude $=25 \mathrm{mV}$; increment potential $=4 \mathrm{mV}$; frequency $=15 \mathrm{~Hz}$; vs. $\mathrm{Ag} / \mathrm{AgCl}$. (b) Cyclic voltammograms measured with bare, GO, rGO, $\mathrm{SnO}_{2}$ nanoparticle, and $\mathrm{SnO}_{2} /$ reduced graphene oxide nanocomposite modified GCE in the solution of $5 \mathrm{mM} \mathrm{Fe}(\mathrm{CN})_{6}^{3-/ 4-}$ containing $0.1 \mathrm{M} \mathrm{KCl}$. Inset: Nyquist diagram of electrochemical impedance spectra for bare and $\mathrm{SnO}_{2} /$ reduced graphene oxide nanocomposite modified GCE in the solution of $5 \mathrm{mM} \mathrm{Fe}(\mathrm{CN})_{6}{ }^{3-/ 4-}$ containing $0.1 \mathrm{M} \mathrm{KCl}$. Adapted from Ref. ${ }^{301}$ with permission of American Chemical Society.

Li et.al. used Nafion and rGO for anodic stripping voltammetric analysis of cadmium with detection limit $0.005 \mathrm{ppb}$. ${ }^{302}$ Willemse et al. reported on the determination of $\mathrm{Cd}^{2+}, \mathrm{Pb}^{2+}$, $\mathrm{Zn}^{2+}$ and $\mathrm{Cu}^{2+}$ by making use of the platform based on the Nafion-graphene nanocomposite film. ${ }^{409}$ LODs of $0.07-0.08 \mathrm{ppb}$ have been achieved for the individual ions, which are comparable to those determined with ICP-MS, and ascribed to a combination of enhanced electron conduction of rGO and the cation exchange capacity of Nafion. Similarly, Chaiyo et al. constructed a Nafion/ionic liquid/graphene electrochemical sensor for simultaneous determination of zinc, cadmium and lead using screen-printed carbon (Figure 20a). ${ }^{303}$ The functionalized graphene-based nanocomposite modified electrode showed better detection performance for $\mathrm{Zn}(\mathrm{II}), \mathrm{Cd}(\mathrm{II})$ and $\mathrm{Pb}(\mathrm{II})$ compared to bare electrode by SWASV (Figure 20b). The detection limits of such sensors for $\mathrm{Zn}(\mathrm{II}), \mathrm{Cd}(\mathrm{II})$ and $\mathrm{Pb}(\mathrm{II})$ detection amounted to $0.09 \mathrm{ng} \mathrm{mL}^{-1}, 0.06 \mathrm{ng} \mathrm{L}^{-1}$ and $0.08 \mathrm{ng} \mathrm{L}^{-1}$, respectively. 
(a)

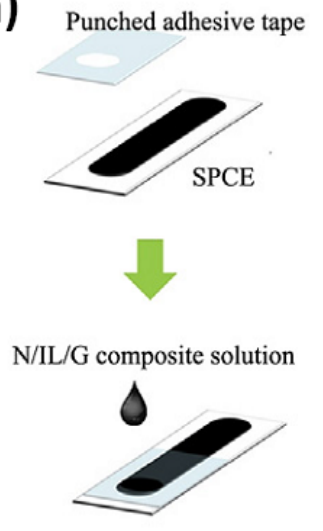

Drop-cast modification (b)

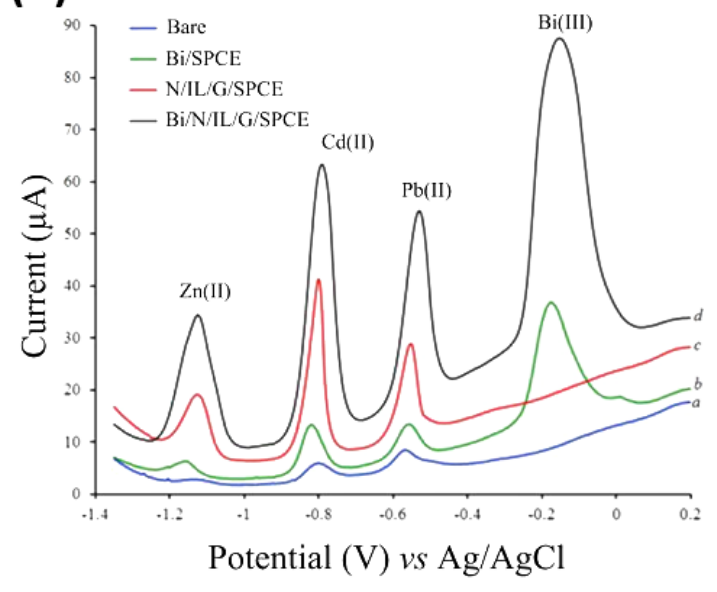

Figure 20. (a) Schematic drawing of the electrochemical sensor fabrication. (b) Square wave anodic stripping voltammetry (SWASV) curves of $50 \mathrm{ng} \mathrm{mL}^{-1} \mathrm{Zn}$ (II), $\mathrm{Cd}$ (II) and $\mathrm{Pb}$ (II) in 0.1 $\mathrm{M}$ acetate buffer solution ( $\mathrm{pH} 4.5$ ). Adapted from Ref. ${ }^{303}$ with permission of Elsevier B.V.

Gong et al. reported ultrasensitive $\mathrm{Hg}(\mathrm{II})$ electrochemical sensor by using monodispersed $\mathrm{Au}$ nanoparticles onto the graphene nanosheet matrix as the enhanced sensing platform. ${ }^{410}$ The detection limit was found to be as low as $6 \mathrm{ppt}$. The interference from other heavy metal ions such as $\mathrm{Cu}^{2+}, \mathrm{Cr}^{3+}, \mathrm{Co}^{2+}, \mathrm{Fe}^{3+}, \mathrm{Zn}^{2+}$ and $\mathrm{I}^{-}$ions associated with $\mathrm{Hg}^{2+}$ analysis could be effectively inhibited. Another example relies on the use of cysteine-functionalized GO (sGO) and carbonyldiimidazole as a cross-linker via the formation of amide and carbamate bonds. The sGO/polypyrrole (PPy) nanocomposite film was grown on the working electrode surface of a screen-printed electrode (SPE) via controlled one-step electrochemical deposition. ${ }^{304}$ The sGO/PPy-SPE was used to detect lead ions in water by differential pulse voltammetry (DPV). The DPV signals were linear in the ranges of 1.4-14 $000 \mathrm{ppb}$ of $\mathrm{Pb}^{2+}$. The measurable detection limit of the sensor is $0.07 \mathrm{ppb}$, being 2 orders of magnitude below the threshold value for drinking water set by the World Health Organization. The average removal efficiency of $\mathrm{Pb}^{2+}$ deposited on the electrode amounted to $99.2 \%$, with relative standard deviation (RSD) of 3.8\%. The selective detection of the GO/PPy composites was investigated by mixing GO/PPy with various metal ions such as $\mathrm{Pb}^{2+}, \mathrm{Na}^{+}, \mathrm{Mg}^{2+}, \mathrm{Cd}^{2+}, \mathrm{Cu}^{2+}, \mathrm{Hg}^{2+}$ and $\mathrm{Ag}^{+}$. The results clearly show that the GO/PPy composites film can selective detection of 
$\mathrm{Pb}^{2+}$ and impassive by the presence of other metal ions. In addition, the developed device can be used multiple times.

TMDs nanostructures were mainly utilized as sensing platforms for the development of electronic and fluorescent sensors, while their electrochemically sensing applications are still limited. Cui et al. synthesized monolayer $\mathrm{MoS}_{2}$ supported $\mathrm{Cu}_{7} \mathrm{~S}_{4}-\mathrm{Au}$ nanocomposite as a sensing platform to detect $\mathrm{Hg}$ (II) by using anodic stripping voltammetric technique. ${ }^{305}$ They found the synergistic effects of both Au domains and active edge sites of monolayer $\mathrm{MoS}_{2}$ played a vital role in the high-performance for detection of $\mathrm{Hg}$ (II) with a limit of detection of $190 \mathrm{nM}$.

In this part of the Review article, we have provided an overview on the recent advances in the metal ion sensing. Different types of 2DMs employed for specific and selective recognition of heavy metals have been discussed in this chapter. By taking advantage of their unique properties 2DMs can be successfully explored to construct a wide range of optical and electrical sensing platforms for the detection of various heavy metal ions. Chemical modification of GO and TMDs has proven to provide promising solution for improving the sensing performance with high specificity, enhanced sensitivity and low detection limit. Chemical metal sensors based on 2DMs have demonstrated high sensitivity detection of a wide variety of heavy metal ions at low concentrations, due to the maximum sensor surface area per unit volume. Their favourable structural and compositional synergy allows them to be excellent electrode materials for fabricating various fluorescent and electrochemical sensing platforms, such as FET-based sensors. Moreover, electrochemical measurements have shown numerous advantages for trace heavy-metal detection, including rapid analysis, good selectivity, and sensitivity. Optical detection systems are other alternatives to the electrochemical detection methods. These represent attractive analytical tools whenever continuous monitoring and real-time information is desired. 


\section{4 (Bio)molecular sensors}

\section{1 (Bio)molecular sensing with graphene, graphene oxide and related composites}

Ultrafast sensing of chemically and biologically active molecules at a low concentrations is critical in a wide range of research fields and in particular for applications, such as chemical analysis $^{411}$ and healthcare, ${ }^{412,413}$ monitoring the environment or diagnostic diseases. ${ }^{414}$ Because of their particular physico-chemical properties, graphene-based materials have been used to fabricate various types of chemical sensors including electrochemical, ${ }^{72,} 413,415-444$ FET, ${ }^{445-449}$ fluorescent ${ }^{450-457}$ and surface enhanced Raman spectroscopy-based sensors. ${ }^{411,458-}$ ${ }^{460}$ Graphene based materials have been used to develop various types of sensors to detect molecules such as glucose, ${ }^{72,415-423,425,427,445,461-465}$, DNA, 72, 428, 430, 447, 449, 451, 452, 455, 457, 466-469 hydrazine, ${ }^{431}, 470-473$ dopamine, ${ }^{72,413,424,432-439,441,454,474,475}$ ascorbic acid, ${ }^{72,413,434,441,476,477}$ $\mathrm{H}_{2} \mathrm{O}_{2},{ }^{72,}{ }^{443,}{ }^{478-480}$ or other aromatic molecules (Figure 21). ${ }^{411,453,456,458,459,} 478$ Graphenebased materials offer various advantages, when compared to other carbon-based nanomaterials like CNTs or fullerenes. In particular, graphene can be easily produced from bulk graphite via exfoliation. ${ }^{126,481-483}$ Moreover, the particular strength of graphene, quality of its crystal structure, as well as its band structure and high conductivity allows the preparation of devices with extremely low noise levels and relatively low $1 /$ f noise. $^{448}$

\subsubsection{Electrochemical (bio)molecular sensors}

Electrochemical sensing is an effective and powerful technique used for qualitative and quantitative determination of bioactive and functional molecules. ${ }^{427}, 465,475$ Numerous advantages including low limit of detection, high selectivity and detection in the presence of interferences made graphene-based materials appealing for fabrication of electrochemical sensors. Because of these reasons, electrochemical sensors belong to the most widespread graphene-based sensors in recent years. The most common approach relies on the use of electrodes based on graphene $e^{442,477}$ or modified graphene-based nanostructures. ${ }^{441,474,478}$ Fast electron transfer and effective electrocatalytic activity of graphene allows the effective detection of target molecules while oxidation. ${ }^{484}$ Among its numerous physico-chemical properties, graphene has wide electrochemical potential window (ca. $2.5 \mathrm{~V}$ in phosphate 
buffer) ${ }^{72}$ and is characterized by lower charge transfer resistance (comparing with glass electrode $)^{72}$ and well characterized redox potentials against most common redox couples. ${ }^{485}$ Keeley et al. developed an electrochemical sensor based on graphene exfoliated in DMF for ascorbic acid (AA) detection. ${ }^{477}$ The electrode was prepared by drop-casting centrifuged graphene supernatant solution onto pyrolysed photoresist film. Cyclic voltammetry investigation revealed wide range of linearity and limit detection up to $0.12 \mathrm{mM}$. Another effective electrode was prepared by mixing platinum nanoparticle and graphene nanosheet. ${ }^{478}$ Such hybrid system displayed the capacity to detect traces of trinitrotoluene (TNT), a typical explosive compound, with detection limits as low as $0.3 \mathrm{ppm}$ and satisfactory reproducibility. Luo et al. presented electrochemical glucose sensor based on $\mathrm{Cu}$ decorated graphene sheets. ${ }^{421}$ As prepared electrode exhibited a low detection limit of $0.5 \mu \mathrm{M}$ and very fast response $(<2 \mathrm{~s})$ and its response linearity up to $4.5 \mathrm{mM}$. Additionally, a synergistic effect of copper and graphene toward glucose oxidation was observed.

As aforementioned, the majority of graphene-based sensors rely on the chemical modification of GO, which exhibits numerous oxygen-rich functional groups that can interact via dipoledipole or strong electrostatic interactions with (charged) molecules, or it can be covalently functionalized with functional molecules, enhancing the occurrence of adsorption events. In particular, covalent grafting of organic molecules on GO offers the flexibility for various functionalizations to enhance the sensor performance. Moreover, the combination of its abundant structural defects and chemical groups facilitates the charge transfer and thus ensure high electrochemical activity. Noteworthy, the chemical and electrical properties of rGO are highly tuneable and can be engineered through control of the reduction process.

Zhou et al. exploited a chemically reduced GO modified glassy carbon electrode (CRGO/GC) for electrochemical sensing of free purine and pyrimidine acids and several other biologically active molecules. ${ }^{72}$ It was demonstrated that CR-GO/GC electrode provides greater electrocatalytic activity to guanine $(\mathrm{G})$, adenine $(\mathrm{A})$, thymine $(\mathrm{T})$ and cytosine $(\mathrm{C})$ oxidation than commonly used glassy carbon (GC) and graphite/glassy carbon graphite/GC electrodes (Figure 21). Moreover, the analytical performance of glucose, ethanol, dopamine and other biological molecules detection was enhanced; e. g. compared with graphite/GC and GC electrodes the CR-GO/GC electrode. In particular, while amperometric measurement of reduced nicotinamide adenine dinucleotide (NADH) exhibited faster response time, wider linear range $(40-800 \mu \mathrm{M})$, lower detection limit $(10.00 \mu \mathrm{M})$ and higher sensitivity $(2.68 \mu \mathrm{A}$ $\mathrm{mM}^{-1} \mathrm{~cm}^{-2}$ ). Gao and co-workers carried out the chemical reduction of GO obtaining 
hydroxyapatite/rGO composite used as effective electrochemical sensor of hydrazine. ${ }^{472}$ The composite dispersed in 1\% acetic acid was applied on GCE and dried resulting in highperformance electrode with outstanding performance of electrocatalytic oxidation of $\mathrm{N}_{2} \mathrm{H}_{4}$. The as-prepared electrode exhibited synergic effect of components during detection of hydrazine, being greater than the electrodes fabricated with rGO and hydroxyapatite separately. Wang and co-workers devised a facile approach relying on the efficient preparation of nitrogen-doped graphene via nitrogen plasma treatment of chemically synthesized graphene, which exhibits excellent electrocatalytic activity toward hydrogen peroxide reduction. ${ }^{415}$ Detection of $\mathrm{H}_{2} \mathrm{O}_{2}$ as one of the products of catalytic glucose oxidation can be applied for selective and sensitive glucose determination during enzymatic processes. Noteworthy, N-doped graphene electrode exhibited high concentration-dependent response and was able to detect up to $0.01 \mathrm{mM}$ of glucose in the presence of interferences. N-doped electrodes can also find their use in detection of important biomolecules such as ascorbic acid (AA), dopamine (DA) and uric acid (UA). ${ }^{413}$ Efficient oxidation and large peak separation provides simultaneous determination of those three biomolecules with detection limits up to $2.2 \cdot 10^{-6}, 2.5 \cdot 10^{-7}$ and $4.5 \cdot 10^{-8} \mathrm{M}$ for AA, DA and UA, respectively. Furthermore, differential pulse voltammetry (DPV) experiment revealed that electrochemical response of target biomolecules increases linearly with increase of molecules concentrations.

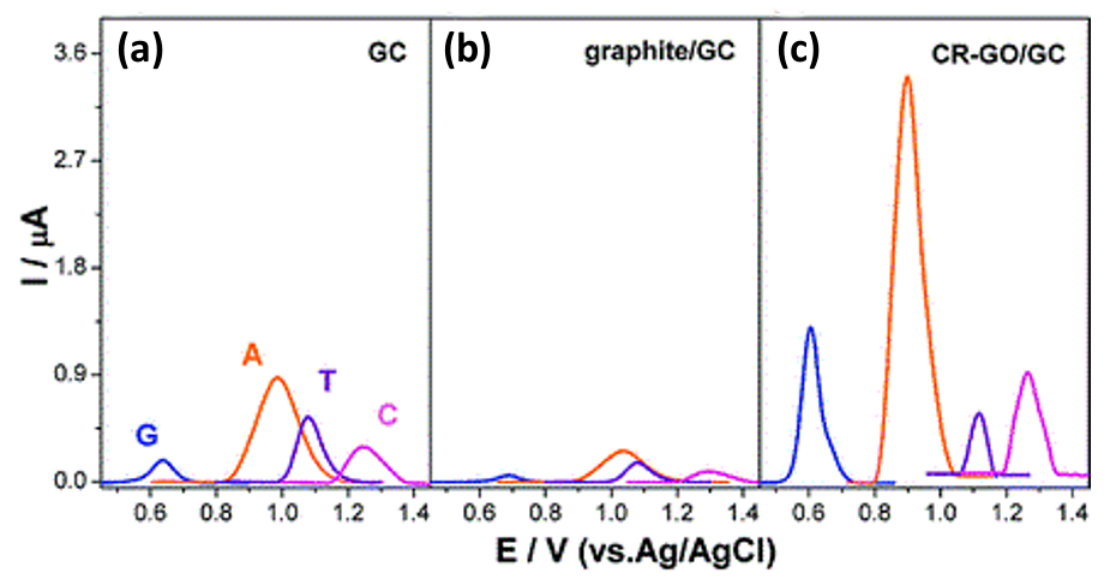

Figure 21. Differential pulse voltammograms (DPVs) recorded by using a) GC electrode, b) graphite/GC electrode, and c) CR-GO/GC electrode, for guanine (blue), adenine (orange), thymine (violet), and cytosine (magenta), respectively. Adapted from Ref. ${ }^{72}$ with permission of American Chemical Society. 


\subsubsection{Field-Effect Transistors for (bio)molecular sensors}

Due to their high selectivity, fast response and excellent limit of detection, graphene-based FET-based sensors became very popular for label-free ultrasensitive biomolecule sensing. By and large, graphene-based materials are used as conducting component in the channel between drain and source electrodes, and subsequently gate potential is applied. ${ }^{446}$ Two different approaches are being pursued for construction of FET sensors, i.e., back gating (usually $\mathrm{Si} / \mathrm{SiO}_{2}$ thin layer) ${ }^{445,446}$ or top gating (electric double layer in electrolyte) ${ }^{449,}{ }^{465}$ also known as solution gated graphene transistors (SGGTs). Both methods have been schematically presented in Figure 22. In general, charged molecules can induce an effective gating field, which influences the conductance of the channel by balancing the charge transfer and gating effect. The change in current as measured allows the analyte determination.
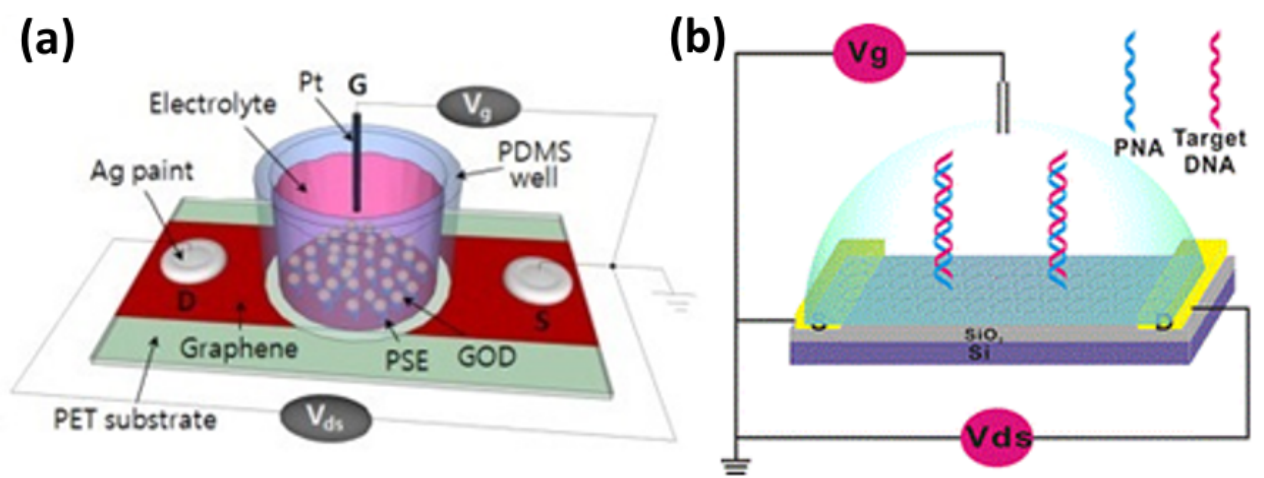

Figure 22. Schematic representation: (a) Graphene top gate FET sensor. Adopted from Ref. 446 with permission of the American Chemical Society. (b) Graphene oxide back-gate FET sensor. Adapted from Ref. ${ }^{449}$ with permission of Elsevier.

Huang and co-workers presented CVD-grown graphene FET sensor functionalized with specific redox mediators for glucose and glutamate detection. ${ }^{445}$ Glucose sensing is usually based on an enzymatic reaction catalysed by glucose oxidase. Since the products of oxidation process are $\mathrm{H}_{2} \mathrm{O}_{2}$ and gluconic acid, direct measurement of $\mathrm{H}_{2} \mathrm{O}_{2}$ is useful for glucose detection. The detection limits were found being at $0.1 \mathrm{mM}$ and $5 \mu \mathrm{M}$ for glucose and glutamate, respectively. Another CVD-grown glucose sensor was assembled by Kwak et $a l .{ }^{446}$ Solution-gated field-effect transistor (SGFET) was constructed utilizing graphene channel modulated by the gate potential applied from the top gate electrode and transmitted through the solution. Such SGFET sensor was exploited to detect glucose in the range of 3.3- 
$10.9 \mathrm{mM}$. Moreover, it provided high resolution and continuous real-time monitoring. To reach the largest sensing response, graphene transistors are operated at the point of maximum transconductance, which leads to large noise that influences the device sensitivity. To avoid such phenomenon $\mathrm{Fu}$ and co-workers exploited the sensing properties of single layered graphene near its neutrality point. ${ }^{447}$ This approach led to a significant decrease of signal-tonoise ratio, thereby making it possible to observe positive signals coming from single stranded DNA (ssDNA) at level of picomolar concentrations (pM). Moreover, to target specific hybridization corresponding with HIV-virus related ssDNA, functionalization of GFET surface with pyrene-linked peptide nucleic acid (pPNA) was performed. Noteworthy, the $1 / \mathrm{f}$ noise in graphene based FET sensors was found to vary with the number of graphene layers, therefore subsequent optimization of this class of sensors is anticipated. ${ }^{448}$ Another approach in which hydrazine-reduced $\mathrm{GO}$ was used to fabricate FET sensor on $\mathrm{SiO}_{2} / \mathrm{Si}$ substrate was proposed by Cai et al. ${ }^{449}$ DNA sensor was prepared by drop-casting rGO suspension onto sensing channel as the conducting material. 1-Pyrenebutanoic acid succinimidyl ester (PASE) used as molecular linker was fixed on the graphene surface via $\pi-\pi$ stacking interactions and peptide nucleic acid (PNA) molecules were covalently anchored. Subsequently, a complementary DNA was applied onto device and specific hybridization caused the shift of $\mathrm{I}_{\mathrm{ds}}-\mathrm{V}_{\mathrm{g}}$ curves and allowed the measurement. The detection limit was estimated being as low as $100 \mathrm{fM}$.

\subsection{3 (Bio)molecular Fluorescence Sensors}

The use of fluorescence as readout in the detection of chemical species and biomolecules holds potential for low-cost, effective and highly sensitive device applications. Graphene/graphene oxide are known to be excellent quenching materials therefore graphenebased materials became suitable for sensing via influence on fluorescence properties. ${ }^{469}$ One of the approaches bases on FRET and is useful for quantitative determination of target molecules. Typically, this kind of set up consists of a donor, an acceptor and a bridge. The acceptor such as GO can effectively quench fluorescence of the donor. The addition of specific molecules or surface modification may cause change in the fluorescence intensity and can be utilized as a sensor assuming the phenomenon is correlated with concentration (Figure 23a-b). ${ }^{450,452}$ 


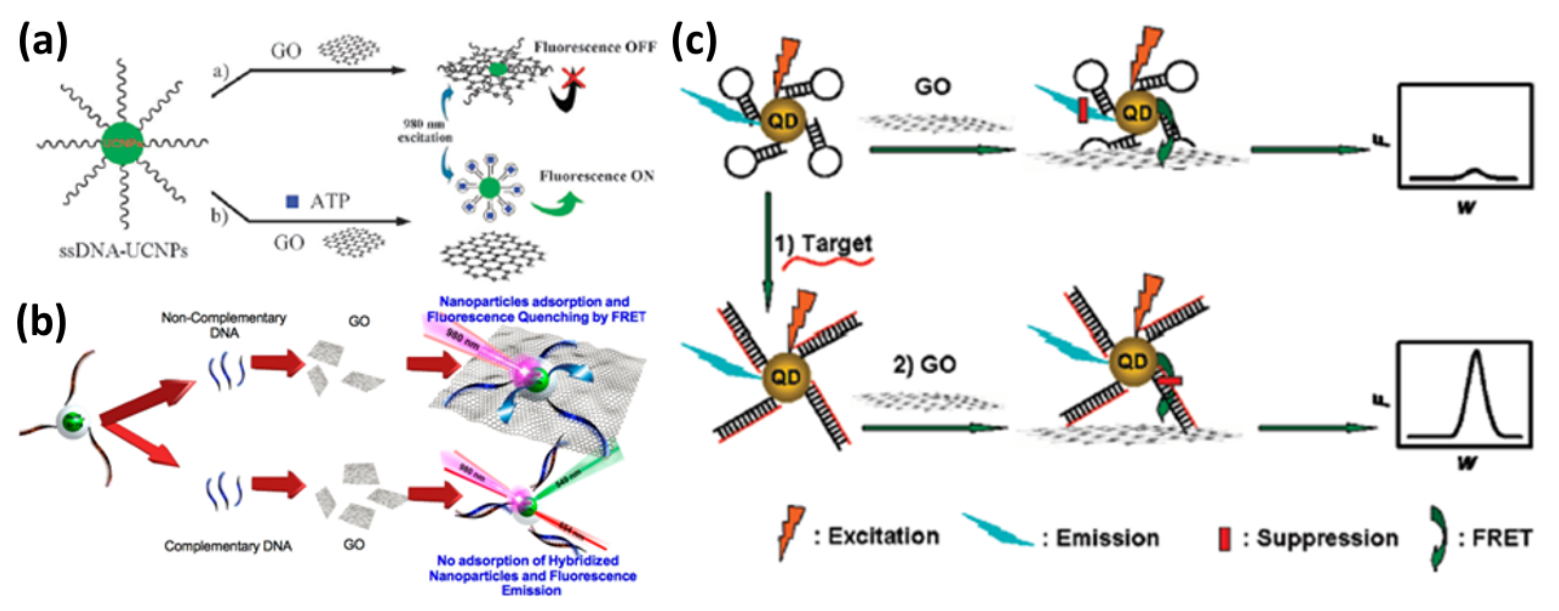

Figure 23. Schematic representation of different FRET mechanisms used in biomolecules sensing. (a) Schematic representation of the upconversion fluorescence resonance energy transfer used for ATP sensing. (Figure adapted from Ref. ${ }^{452}$ with permission of the Royal Society of Chemistry.) (b) Depending on the presence of complementary DNA emission is observed or completely quenched, detection of specific DNA hybridization. Adapted from Ref. ${ }^{450}$ with permission of American Chemical Society. (c) DNA sensing. Molecular beacon decorated QDs quenched by GO platform, in the presence of complementary DNA emission is significantly enhanced. (Figure adapted from Ref. ${ }^{451}$ with permission of American Chemical Society.)

Dong and co-workers reported on the FRET from quantum dots (QDs) to GO. ${ }^{451}$ In particular, the authors demonstrated that QDs substituted with molecular beacon (MB) as a recognition unit towards targeted molecule can strongly interact with GO surface, to allow the design of novel sensitive and selective platforms for fluorescence-quenching detection of DNA. In general GO's quenching properties were used to decrease fluorescence intensity in the presence of QDs - an effective donor supplied with MB that provides efficient energy transfer to GO (Figure 23c). Fluorescent graphene quantum dots (GQDs) were also applied for detection of aromatic nitro compounds (e.g. TNT) working as a quenchers in a wide range sustaining linear correlation. ${ }^{453}$ Another DNA sensor using FRET between upconversion nanoparticles and GO was reported by Alonso-Cristobal et al. ${ }^{450}$ Correlation between concentration of ssDNA (Figure 24) and luminescence intensity was presented. Effective detection limit was experimentally shown to be in the picomolar range. 

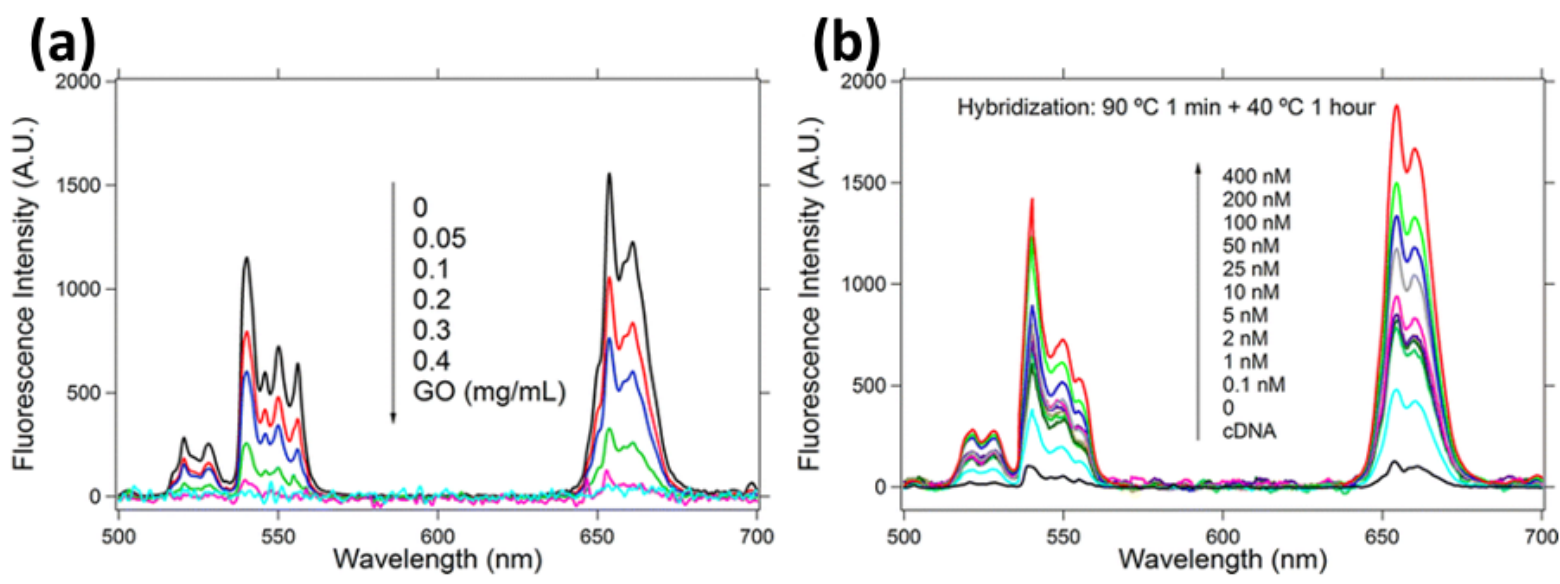

Figure 24. Up-conversion fluorescence spectra of the UCNPs@ $\mathrm{SiO}_{2}-\mathrm{ssDNA}$ nanoparticles $\left(0.4 \mathrm{mg} \mathrm{mL}^{-1}\right)$ a) in the presence of different concentrations of $\mathrm{GO}$, and b) in the presence of different concentrations of complementary ssDNA and $0.3 \mathrm{mg} \mathrm{mL}^{-1}$ of GO. Adapted from Ref. ${ }^{450}$ with permission of American Chemical Society.

GO-based platform for DNA and proteins sensing reported by Lu and co-workers includes ssDNA supplied with fluorescent organic dye that strongly adsorbs on GO's surface simultaneously entailing high quenching efficiency. ${ }^{455}$ The addition of complementary ssDNA sequence causes efficient fluorescence increase and may be used to determine desired nucleotide sequences. Experiments also proved the dependence on different proteins concentrations. The results revealed that the presence of human thrombin might be determined without the interference of other proteins.

\subsection{4 (Bio)molecular Sensing via Surface Enhanced Raman Scattering}

Surface Enhanced Raman Scattering represents a most powerful analytical technique for sensitive chemical sensing, which became an attractive solution for detection of aromatic compounds. Typically, weak Raman signals are intensified through enhancement of the local electromagnetic field, occurring with the charge transfer between adsorbed molecules and metal surface. Consequently, to observe SERS signal, molecules must be adsorbed or be placed close to a metal surface. The most common approach bases on modification of graphene-based material surface with metallic nanoparticles (NPs). ${ }^{411}{ }^{459}$ Optimum size, 
shape, charge and surface chemistry of NPs are being taken into account to project non-toxic and effective sensors. ${ }^{104}$

Lu et al. proposed the use of Ag and Au NP-decorated single-layer rGO film anchored on $\mathrm{Si}$ surface to detect popular organic dyes rhodamine 6G (R6G), methyl violet (MV), rhodamine $\mathrm{B}$ (RB) and methylene blue (MB) at nanomolar concentrations. ${ }^{411}$ Such molecules easily adsorb on rGO surface while Ag and Au nanoparticles provide dramatic enhancement in the Raman intensities coming from the sample. Moreover, a correlation between NPs size and observed signals have been monitored, and it could be controlled through the reaction time. Another modification with Ag NPs for ultrasensitive detection of aromatic molecules was demonstrated by Liu and co-workers. ${ }^{458}$ Additional enhancement of the Raman signals was ensured by GO treated with 3-mercaptopropyltrimethoxysilane (MPTMS), further functionalized with Ag-NPs. An effective detection of differently charged molecules was successfully characterized and presented. In particular, experiments with $\mathrm{PPh}_{3}$ used as target molecule exhibited limit of detection as low as $10^{-9} \mathrm{M}$.

Graphene grown on copper foil and Ag NPs anchored on Si substrate can also be used to prepare SERS active substrate for detection of TNT by alkaline hydrolysis. ${ }^{459}$ High $\mathrm{pH}$ values provide efficient hydrolysis of TNT and enhance intensity in Raman spectra signals. Extremely high limit of detection (up to $6.6 \cdot 10^{-10} \mathrm{M}$ ) and excellent anti-interference ability confirms perspective potential of SERS sensors. Shanta and Cheng prepared hydrazine reduced GO combined with silver nanoprisms and utilized such hybrid system for trace detection of tetrachlorobiphenyls (PCBs). ${ }^{460}$ As prepared chip successfully allowed for multiplex measurement of several environmentally important aromatic compounds. Proposed SERS sensor provided low limit of detection (up to $100 \mathrm{nM}$ ) and simultaneous analysis of multiple isomers.

Values such as limit of detection and linearity range for detection of vary biomolecules have been summarized in Table 9.

Table 9. Comparison of sensing properties of different graphene-based sensors.

\begin{tabular}{|c|c|c|c|c|c|}
\hline Material & Type of sensor & Analyte & $\begin{array}{c}\text { Limit of } \\
\text { detection }\end{array}$ & $\begin{array}{c}\text { Linearity } \\
\text { Range }\end{array}$ & Ref. \\
\hline$D M F$-exfoliated & electrochemical & AA & $0.12 \mathrm{mM}$ & $0.4-6.0 \mathrm{mM}$ & 477 \\
\hline
\end{tabular}




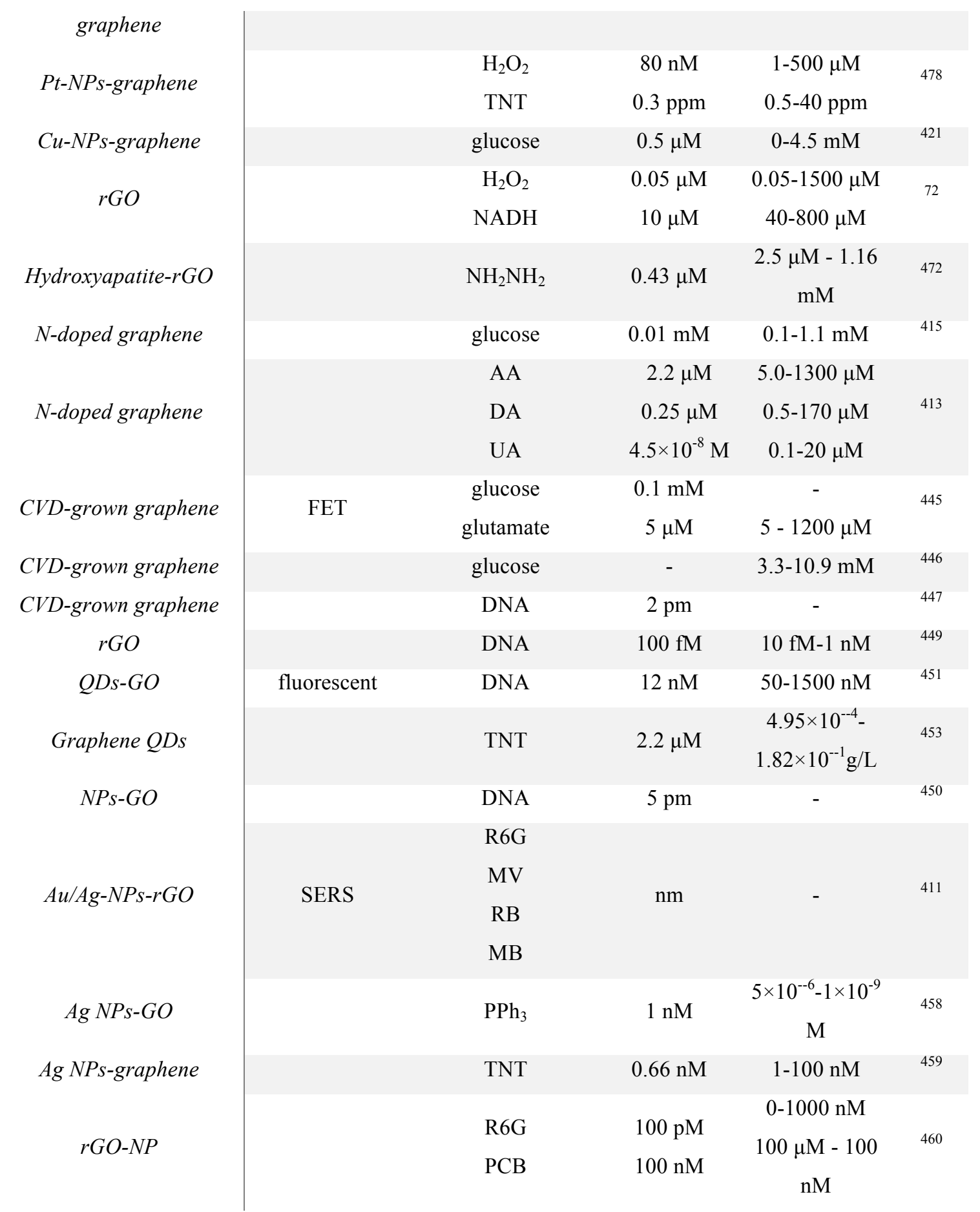

List of abbreviations. DMF : dimethylformamide, AA : ascorbic acid, NPs : nanoparticles, TNT : 2,4,6trinitrotoluene, $\mathrm{NADH}: \beta$-nicotinamide adenine dinucleotide, $\mathrm{NH}_{2} \mathrm{NH}_{2}$ : hydrazine, $\mathrm{DA}$ : dopamine, UA : uric acid, QDs : quantum dots, SERS : Surface Enhanced Raman Scattering, R6G : rhodamine 6G, MV : methyl violet, $\mathrm{RB}$ : rhodamine $\mathrm{B}, \mathrm{MB}:$ methylene blue, $\mathrm{PPh}_{3}$ : triphenylphosphine, $\mathrm{PCB}: 2,2^{\prime}, 3,3^{\prime}$-tetrachlorobiphenyl. 


\section{2 (Bio)molecular sensing with Transition Metal Dichalcogenides}

TMDs were recently used to develop a wide range of chemical sensor including electrochemical, ${ }^{132,134,486-500}$ fluorescent $^{99,133,501-503}$ and field-effect transistor sensors. ${ }^{504-506}$ $\mathrm{MoS}_{2}$ and its analogues like tungsten disulphide $\left(\mathrm{WS}_{2}\right)$, tantalum disulphide $\left(\mathrm{TaS}_{2}\right)$, tungsten diselenide $\left(\mathrm{WSe}_{2}\right)$ as well as ternary chalcogenides are widely investigated with promising results in chemical sensing applications. ${ }^{132,500-502,507}$

Among various TMDs, $\mathrm{MoS}_{2}$ is the most studied material. The interaction of $2 \mathrm{D}$ lattice of $\mathrm{MoS}_{2}$ with charged molecules efficiently influences its electrochemical properties, which can be measured directly with voltammetric measurements. ${ }^{490,}$ 492, 493 Furthermore, $\mathrm{MoS}_{2}$ nanosheets used for electrode preparation exhibit significant electrocatalytic activity toward a range of important analytes, including glucose, ${ }^{493,}{ }^{497} \mathrm{H}_{2} \mathrm{O}_{2},{ }^{488}$ dopamine, ${ }^{494,}$ 495, 508 ascorbic acid, ${ }^{508}$ tryptophan ${ }^{509}$ and DNA. ${ }^{489,503,506}$ Another advantage of $\mathrm{MoS}_{2}$ is its feasibility to form composites which enlarges a wide range of properties and application potential. ${ }^{509,510}$ Remarkably, the presence of noble metal nanostructures effectively enhances electrochemical detection of target molecules. Upon mixing $\mathrm{MoS}_{2}$ with of NPs (e.g. Pt, Ag, Au) improves electron transfer and allows achieving lower limits of analyte detection. ${ }^{495}, 508,509 \mathrm{MoS}_{2}$ is also an excellent quenching material, thus can be applied for highly sensitive detection of biomolecules via fluorescence phenomenon. ${ }^{99,511}$ Moreover $\mathrm{MoS}_{2}$ occurs high affinity toward ssDNA, which is supported by van der Waals interactions, while double stranded DNA (dsDNA) formed in the presence of complementary sequence, hardly interacts with its twodimensional surface. Different mechanisms of molecular detection utilizing TMD's based materials are presented on Figure 25.

$\mathrm{Wu}$ and co-workers presented controllable lithiation process of $\mathrm{MoS}_{2}$ and subsequent liquidphase exfoliation of the intercalated structure in water or ethanol. The electrochemical study of as-prepared $\mathrm{MoS}_{2}$ nanosheets revealed a reduction peak in the $\mathrm{CV}$ in $\mathrm{NaCl}$ aqueous solution. The reduced $\mathrm{MoS}_{2}$ displayed good conductivity and fast electron transfer rate in the most common redox systems applicable in electrochemical sensing of glucose and dopamine (DA) in the presence of ascorbic acid (AA) and uric acid (UA) ${ }^{486}$ GCE was covered with $\mathrm{MoS}_{2}$ using 3-aminopropyltriethoxysilane (APTES) as linking molecule. The electrode prepared with such hybrid material yielding voltagrams displaying good peak separation, thus allowing the detection of dopamine maintaining linearity in the range of $1-50 \mu \mathrm{M}$ in the 
presence of interferences (AA) and (UA). Additional functionalization of electrode with glucose oxidase enables effective glucose detection.

Another approach for molecular detection was introduced by Huang et al. ${ }^{497} \mathrm{MoS}_{2}$ nanosheet obtained via liquid-phase exfoliation in ethanol/water mixed with Ni NPs and drop-cast onto GCE and naftion copolymer. The as-prepared sensors exhibited amperometric response toward glucose with detection limit as low as $0.31 \mu \mathrm{M}$. Such GCE/Ni-MoS $/ \mathrm{Naftion}$ electrode allowed nonenzymatic detection of glucose with good reproducibility and long-term storage stability. The simultaneous detection of DA, AA and UA investigated by Sun et al. by using $\mathrm{MoS}_{2}-\mathrm{Au}$ NPs decorated electrode to achieve ultrasensitive determination of aforementioned biologically important molecules. ${ }^{508}$ The joint effect of a wide linearity range and effective electrical response yielded sensing ability with detection limits as low as 100 $\mu \mathrm{M}, 0.05 \mu \mathrm{M}$ and $10 \mu \mathrm{M}$ for AA, DA and UA, respectively. Zhu and co-workers have shown the fluorescence quenching ability of $\mathrm{MoS}_{2} \cdot{ }^{99}$ Nanosheets obtained through exfoliation of bulk $\mathrm{MoS}_{2}$ using electrochemical lithium-intercalation method were used as nanoprobes for adenosine. $\mathrm{MoS}_{2}$ supplied with probe molecule occurs high fluorescence quenching efficiency (up to $98 \%$ comparing with probe molecule intensity). Interaction with adenosine observed as intensity increase can be used as highly selective sensor able to detect up to $5 \mu \mathrm{M}$ of adenosine. Another $\mathrm{MoS}_{2}$ fluorescent sensor for DNA detection was proposed by Huang et $a l .{ }^{503}$ The nanosheets were prepared by using solution exfoliation method. The authors presented selective target DNA recognition via hybridization chain reactions (HCRs) with stunning detection limit of $15 \mathrm{pM}$. Another example of using adsorption of dye-labelled ssDNA on $\mathrm{MoS}_{2}$ surface was presented by Huang et. al. Chemically exfoliated $\mathrm{MoS}_{2}$ was utilized as biosensing platform for complementary DNA and allowed detection up to 500 pM. $^{512}$ Shan et. al. exploited $\mathrm{MoS}_{2}$-based FET for glucose detection. ${ }^{513}$ The device was fabricated on $\mathrm{Si} / \mathrm{SiO}_{2}$ substrate with $\mathrm{Au} / \mathrm{Ni}$ drain electrodes. The sensor exhibited very fast response time $(<1 \mathrm{~s})$ and linearity in the range of $300 \mathrm{nM}-30 \mathrm{mM}$, which corresponds to average glucose concentration present in the blood consisting potential candidate for realtime detection of glucose concentration in human blood. 
(a)

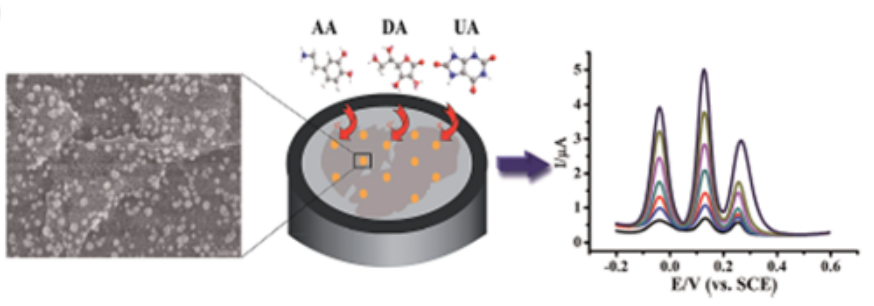

(b)

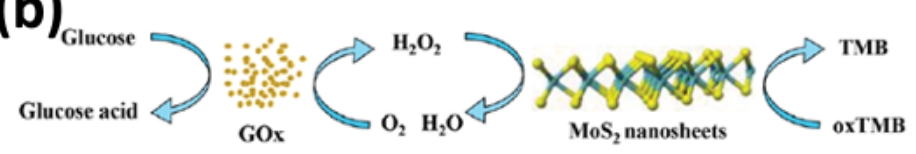

(c)

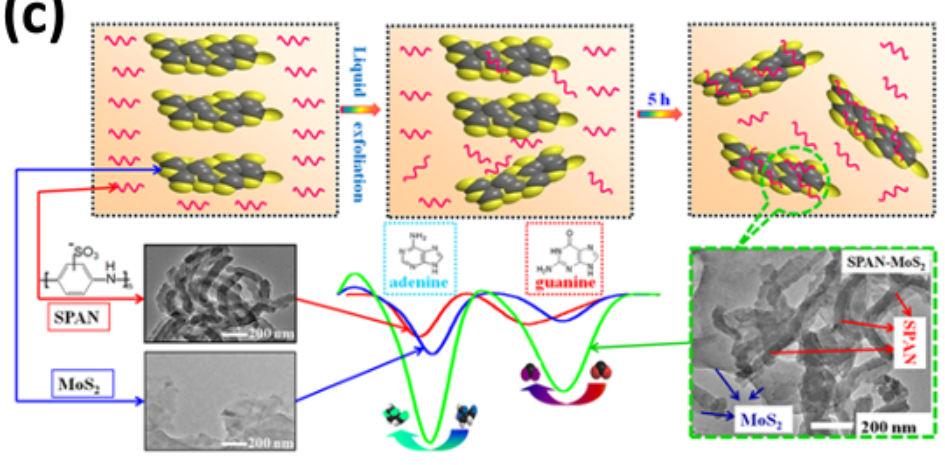

(d)

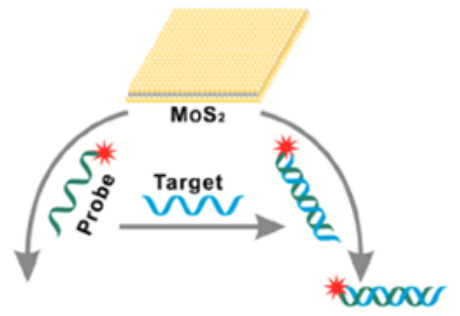

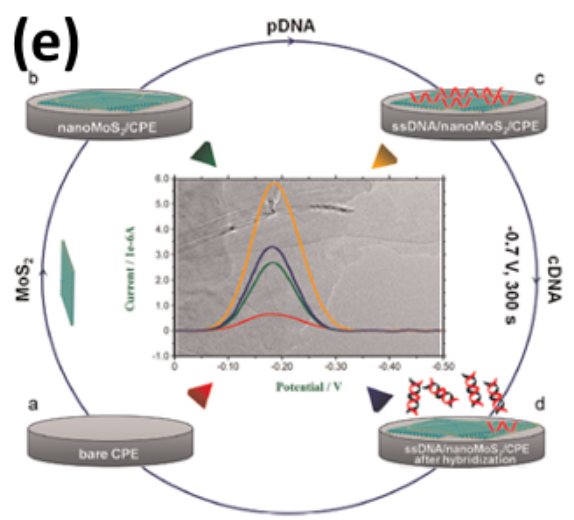

Figure 25. Representation of different mechanisms used for chemical detection utilizing $\mathrm{MoS}_{2}$-based materials. (a) Electrochemical sensing of ascorbic acid (AA), dopamine (DA) and uric acid (UA) with gold nanoparticle- $\mathrm{MoS}_{2}$ nanosheets. Reproduced with permission. ${ }^{508}$ Copyright 2014, Royal Society of Chemistry. (b) Sketch of catalytic reactions while colorimetric detection of glucose. Adapted from Ref. ${ }^{499}$ with permission of the Royal Society of Chemistry. (c) SPAN-MoS 2 for electrochemical detection of adenine and guanine. (Figure adapted from Ref. ${ }^{134}$ with permission of the American Chemical Society). (d) Fluorescent detection of complementary ssDNA.(Figure adapted from Ref. ${ }^{99}$ with permission of the American Chemical Society). (e) Label-free electrochemical sensing of DNA. Adapted from Ref. ${ }^{489}$ with permission of Elsevier.

Inspired by the unique device properties offered by $\mathrm{MoS}_{2}$, other TMDs (including $\mathrm{WS}_{2} \mathrm{TaS}_{2}$, $\mathrm{Ta}_{2} \mathrm{NiS}_{5}$ ) have been the subject of further studies for sensing applications. The group of layered TMCs, including the chalcogenides with well-defined crystal structures have been widely explored. ${ }^{502,514}$ Since the $\mathrm{MoS}_{2}$ is known for its quenching properties, the entire group of TMDs is being considered as effective platform for fluorescent sensing of biomolecules. $^{501,507}$ 
Zhang et al. have used single-layer TMDs nanosheets as fluorescent sensors for DNA detection. ${ }^{501}$ To investigate the quenching ability of $\mathrm{TiS}_{2}$ and $\mathrm{TaS}_{2}$, 6-carboxyfluoresceinlabeled ssDNA probe and target DNA molecule were used. In both cases the dichalcogenides fluorescence intensity was effectively quenched. $\mathrm{TiS}_{2}$ as well as $\mathrm{TaS}_{2}$ exhibited fast detection of complementary DNA with low limit of detection of $0.2 \mathrm{nM}$ and $0.05 \mathrm{nM}$, respectively. Noteworthy, $\mathrm{TaS}_{2}$-based nanosensor was found to be even more sensitive than $\mathrm{MoS}_{2}$ with detection limit of $0.1 \mathrm{nM}$. Another fluorescent sensing platform based on $\mathrm{WS}_{2}$ for T4polynucleotide kinase and its inhibitors was introduced by Ge and co-workers. ${ }^{132}$ The dsDNA does not exhibit strong adsorption on $\mathrm{WS}_{2}$ sheets, yet when phosphorylated and degraded the ssDNA binds to nanosheets as evidenced by the occurrence of fluorescence quenching. Linearity, low limit of detection and low signal-to-background ratio make this material promising for application in monitoring of biological processes. Huang et al. developed $\mathrm{WS}_{2}$ ink by sonication-assisted liquid exfoliation in water supported by $\mathrm{Au}$ nanoparticles for detection of $17 \beta$-estradiol. ${ }^{496}$ Electrode prepared by covering GCE by $\mathrm{WS}_{2}$, then $\mathrm{Au} \mathrm{NPs}$ were anchored and ssDNA aptamer was applied for selective detection of target molecule. As prepared electrode allowed to determine $17 \beta$-estradiol at concentration as low as $2 \mathrm{pM}$. Moreover they proved that only target molecule may cause such obvious current change at concentration in range of $10^{-10} \mathrm{M}$. Tan and co-workers prepared single-layer ternary chalcogenide for fluorescence sensing of DNA. ${ }^{502}$ Microsized crystalline flakes of $\mathrm{Ta}_{2} \mathrm{NiS}_{5}$ were obtained via chemical vapour transport technique from their elementary powders. Then high yield exfoliation was performed to produce single-layered compound. Probe DNA was supplied with Texas red fluorescent reporter functionalized with the probe DNA was employed since it is known to be efficiently quenched when interaction with TMDCs. The fluorescence could be recovered when the target ssDNA was added to form dsDNA. The fabricated sensor exhibited high sensitivity (up to $50 \mathrm{pM}$ ) and affordable detection time of 10 min. $\mathrm{Ta}_{2} \mathrm{NiS}_{5}$ was also used as "Capture-Release" nanosensor for detection of biological species (Figure 26). 

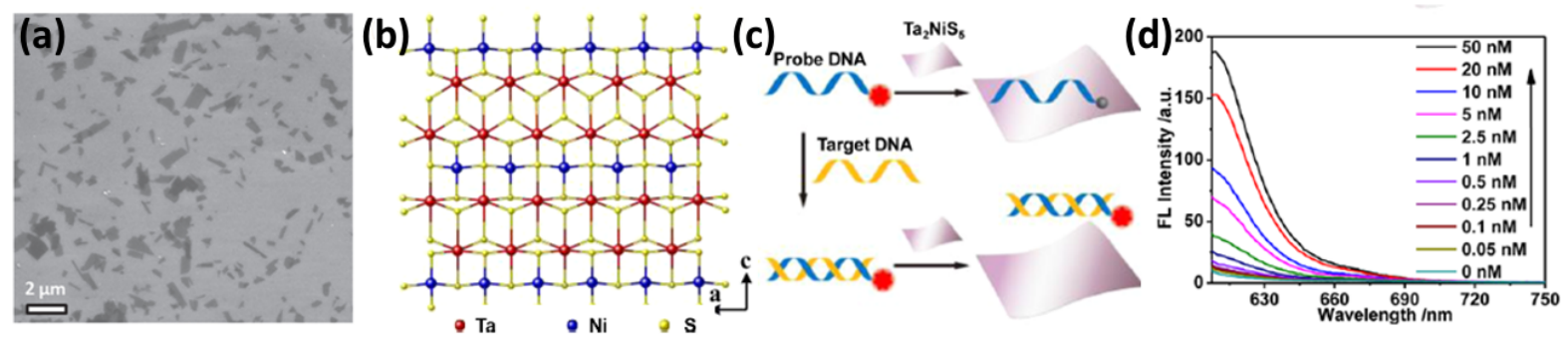

Figure 26. a) Scanning Electron Microscopy (SEM) image of $\mathrm{Ta}_{2} \mathrm{NiS}_{5}$ nanosheets. b) Crystal structure of $\mathrm{Ta}_{2} \mathrm{NiS}_{5}$ along the b-axis. c) Schematic representation of quenching ability of $\mathrm{Ta}_{2} \mathrm{NiS}_{5}$ applied for ssDNA detection. d) Fluorescence detection of different concentrations of target molecule in the presence of probe ssDNA. Adapted from Ref. ${ }^{502}$ with permission of American Chemical Society.

\section{3 (Bio)molecular sensing with TMDs-graphene hybrid materials}

Both TMDs and graphene exhibit a range of physico-chemical properties suitable for various electronic and sensing applications. In particular, further modifications of $\mathrm{MoS}_{2}$ and generation of hybrid materials might be used to generate composites with desired properties. The synergistic effect on electrochemical performance of carbon-based materials with metal or metal oxides have been previously reported ${ }^{515}$ thus suggesting that the combination of $\mathrm{MoS}_{2}$ with graphene-like structures as excellent candidate for a new class of electrochemical sensors. Electrodes made of TMC-graphene composites exhibit significant enhancement of electron transfer conductivity. ${ }^{516-518}$

Huang et al. reported a $\mathrm{MoS}_{2}$-graphene composite prepared via modified L-cysteine-assisted solution-phase method casted on GCE to assemble an electrode. ${ }^{511}$ The composite displayed a wide electrochemical potential window (ca. $2.9 \mathrm{~V}$ in phosphate buffer), which is comparable to graphene-based electrodes. The as-prepared electrode was utilized for voltammetric determination of acetaminophen exhibiting linear response in the range of 1-100 $\mu \mathrm{M}$. Moreover, the hybrid material showed good interference resistance: in the presence of other electrochemically active molecules the value of oxidation peak current was sustained. Another electrochemical sensor composed of $\mathrm{MoS}_{2}$-graphene nanohybrid film was developed by Zhao and co-workers. ${ }^{519}$ The GCE was embedded in $\mathrm{MoS}_{2}$-graphene nanohybrid resulting in significantly enhanced sensitivity towards honokiol - known in view of its antitumor and antibacterial activity used in western medicine. The electrode revealed high selectivity and 
stability with a spectacular limit of detection corresponding to $6.2 \times 10^{-10} \mathrm{M}$. Pramoda et al. performed electrocatalytic activity of $\mathrm{MoS}_{2}-\mathrm{rGO} / \mathrm{GCE}$ against dopamine and proved its superior nature over bare rGO-modified electrode. ${ }^{520}$ Effective detection of DA in the presence of interferences was established at the level of $0.55 \mu \mathrm{M}$.

The most important parameters, including type of sensor, limit of detection and linearity range for TMDs-based sensors are being presented in Table 10.

Table 10. Comparison of sensing properties of different transition metal chalcogenides based sensors.

\begin{tabular}{|c|c|c|c|c|c|}
\hline Material & Type of sensor & Analyte & $\begin{array}{l}\text { Limit of } \\
\text { detection }\end{array}$ & $\begin{array}{c}\text { Linearity } \\
\text { Range }\end{array}$ & Ref. \\
\hline \multirow[t]{5}{*}{$\mathrm{MoS}_{2}$} & electrochemical & $\mathrm{H}_{2} \mathrm{O}_{2}$ & $2.5 \mathrm{nM}$ & $0.1-100 \mu \mathrm{M}$ & 488 \\
\hline & & DNA & $0.019 \mathrm{fM}$ & $0.1-1 \times 10^{5} \mathrm{nM}$ & 489 \\
\hline & fluorescent & DNA & $15 \mathrm{pM}$ & $0-200 \mathrm{pM}$ & 503 \\
\hline & & DNA & $500 \mathrm{pM}$ & $0-50 \mathrm{nM}$ & 512 \\
\hline & FET & glucose & $300 \mathrm{nM}$ & $\begin{array}{c}300 \mathrm{nM}-30 \\
\mathrm{mM}\end{array}$ & 513 \\
\hline \multirow[t]{2}{*}{$\mathrm{MoS}_{2}-\mathrm{Pt} \mathrm{NPS}$} & electrochemical & $\begin{array}{l}\text { DA } \\
\text { AA }\end{array}$ & $\begin{array}{l}0.17 \mu \mathrm{M} \\
0.98 \mu \mathrm{M}\end{array}$ & $\begin{array}{c}0.5-150 \mu \mathrm{M} \\
5-1000 \mu \mathrm{M}\end{array}$ & 495 \\
\hline & & $\mathrm{H}_{2} \mathrm{O}_{2}$ & $1.0 \mu \mathrm{M}$ & $4.0-48500 \mu \mathrm{M}$ & 521 \\
\hline$M o S_{2}-N i N P s$ & & glucose & $0.31 \mu \mathrm{M}$ & $0-4 \mathrm{mM}$ & 497 \\
\hline $\mathrm{MoS}_{2}-\mathrm{Ag} /$ chitosan & & tryptophan & $0.05 \mu \mathrm{M}$ & $0.5-120 \mu \mathrm{M}$ & 509 \\
\hline \multirow[t]{3}{*}{$M o S_{2}-A u N P s$} & & $\begin{array}{l}\text { AA } \\
\text { DA } \\
\text { UA }\end{array}$ & $\begin{array}{c}100 \mu \mathrm{M} \\
0.05 \mu \mathrm{M} \\
10 \mu \mathrm{M}\end{array}$ & $\begin{array}{c}1-70 \mathrm{mM} \\
0.05-4 \times 10^{4} \mu \mathrm{M} \\
10-10^{3} \mu \mathrm{M}\end{array}$ & 508 \\
\hline & & $\mathrm{DA}$ & $80 \mathrm{nM}$ & $0.1-200 \mu \mathrm{M}$ & 494 \\
\hline & & glucose & $2.8 \mu \mathrm{M}$ & $10-300 \mu \mathrm{M}$ & 493 \\
\hline \multirow[t]{2}{*}{$M o S_{2}-P A N I$} & & $\begin{array}{l}\text { adenine } \\
\text { guanine }\end{array}$ & $\begin{array}{l}3 \mathrm{nM} \\
5 \mathrm{nM}\end{array}$ & $\begin{array}{l}0.05-100 \mu \mathrm{M} \\
0.05-100 \mu \mathrm{M}\end{array}$ & 134 \\
\hline & & chloramphenicol & $65 \mathrm{nM}$ & $0.1-1000 \mu \mathrm{M}$ & 498 \\
\hline$W S_{2}$ & & glucose & $2.9 \mu \mathrm{M}$ & $5-300 \mu \mathrm{M}$ & 500 \\
\hline$W S_{2}-A u N P s$ & & $17 \beta$-estradiol & $1 \mathrm{pM}$ & $0.01-10 \mathrm{nM}$ & 496 \\
\hline$W S_{2}$-acetylene black & & DNA & $0.12 \mathrm{fM}$ & $0.001-100 \mathrm{pM}$ & 522 \\
\hline $\mathrm{TaS}_{2}$ & fluorescent & DNA & $0.05 \mathrm{nM}$ & $0-5 \mathrm{nM}$ & 501 \\
\hline $\mathrm{TaNiS}_{5}$ & & DNA & $50 \mathrm{pM}$ & $0-5 \mathrm{nM}$ & 502 \\
\hline \multirow[t]{3}{*}{$M_{0} S_{2}$-graphene } & electrochemical & acetaminophen & $20 \mathrm{nM}$ & $0.1-100 \mu \mathrm{M}$ & 511 \\
\hline & & honokiol & $0.62 \mathrm{nM}$ & $\begin{array}{c}1.0 \mathrm{nM}-2.5 \\
\mu \mathrm{M}\end{array}$ & 519 \\
\hline & & $\mathrm{H}_{2} \mathrm{O}_{2}$ & $1.25 \mu \mathrm{M}$ & $6.25-225 \mu \mathrm{M}$ & 523 \\
\hline
\end{tabular}




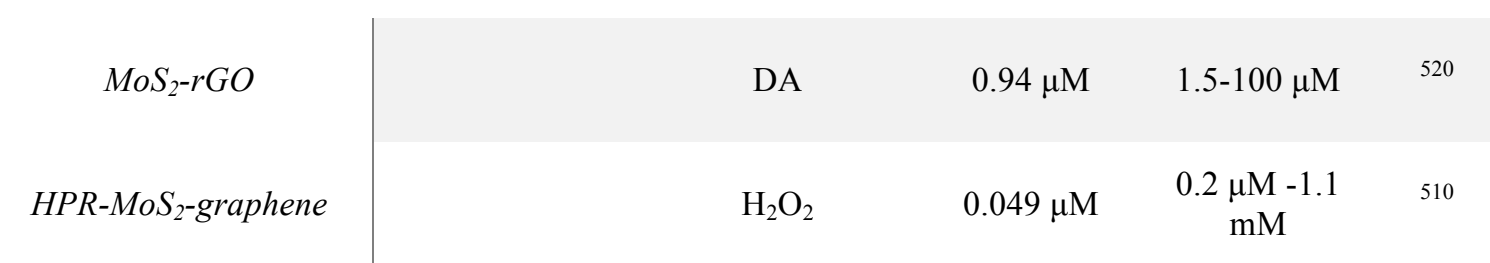

List of abbreviations. NPs : nanoparticles, DA : dopamine, AA : ascorbic acid, UA : uric acid, PANI : polyaniline, $\mathrm{TaS}_{2}$ : tantalum(IV) sulfide, $\mathrm{TaNiS}_{5}$ : tantalum nickel sulfide, HPR : horseradish peroxidase.

\section{4 (Bio)molecular sensing with other 2DMs}

Hexagonal $\mathrm{BN}$ is known to be the most stable allotrope among $\mathrm{BN}$ crystalline forms. A few electron transfer reactions toward biomolecules oxidation for commonly used electrodes immobilized with boron nitride were reported and the results are comparable to other 2DMs based sensors. ${ }^{524}, 525$ However, SERS seems to be more popular technique for the development of hBN-based chemical sensors. Noteworthy, boron-nitrogen bonds of hBN can be used for further modifications or to facilitate the detection of organic molecules via the polarization of B-N bonds. ${ }^{168}$ Moreover, the modification of the BN surface, e.g. introducing noble metal NPs, allows the enhancement in Raman the signal thus rendering such material a practical support for SERS. Moreover, BN exhibits high fluorescence quenching ability and different affinity forward single stranded DNA and double stranded DNA, allowing sensitive detection of complementary DNA fragments. ${ }^{526}$

Khan et al. have reported electrocatalytic behaviour of hBN toward the detection of dopamine. ${ }^{524}$ The electrode was prepared by drop-casting ethanolic solution of crystalline hexagonal boron nitride on screen-printed graphitic electrode (SPE) and occurred to determine dopamine in the presence of common interference molecules ascorbic acid (AA) and uric acid (UA). The obtained sensor exhibited limit of detection of $0.65 \mu \mathrm{M}$, being comparable to other 2D-based dopamine sensors. Zhan and co-workers exploited the quenching ability of boron nitride for the DNA detection. ${ }^{526}$ The experiment revealed effective fluorescence decrease of labelled ss-DNA in the presence of boron nitride nanosheets (BNNS), reaching up to $85 \%$. This sensor exhibited low limit of detection toward target DNA molecule (104 pM) and linearity in the range of 0.6-20 nM. Lin et al. have assembled a SERS-based sensor for rhodamine $6 \mathrm{G}(\mathrm{R} 6 \mathrm{G})$ detection with high reusability. ${ }^{168}$ Silver-decorated boron nitride nanosheets, prepared via reduction of Ag salt in the presence of BNNS, were deposited on quartz substrates using substrate transfer techniques resulting in thermal-oxidation resistant material, which facilitates removal of residue analyte. Another 
SERS sensor for aromatic molecules was prepared by introduction of Au NPs onto BN nanosheets obtained by Scotch tape technique. Cai et al. proposed substrates tested toward R6G detection, with signals greatly enhanced in the presence of $10^{-6} \mathrm{M}$ solutions of analyte. ${ }^{527}$ The fabricated sensor also allows the effective removal of detected molecules by thermal treatment.

MXenes-based sensors exhibit good electrochemical activity toward range of biologically important molecules like glucose, ${ }^{528} \mathrm{H}_{2} \mathrm{O}_{2}{ }^{178,}{ }^{529}$ and nitrites. ${ }^{179}$ Wang and co-workers presented nanocomposite of $\mathrm{TiO}_{2}$ modified with $\mathrm{Ti}_{3} \mathrm{C}_{2} \mathrm{MXene}$ encapsulating haemoglobin with effective activity toward $\mathrm{H}_{2} \mathrm{O}_{2}$ detection. ${ }^{529} \mathrm{TiO}_{2}-\mathrm{Ti}_{3} \mathrm{C}_{2}$ was synthesized in autoclave then the mixture consisting of suspension of nanocomposite, haemoglobin in phosphate buffered saline (PBS) and naftion were applied on GCE by simple casting method. The obtained MXene enzyme-based sensor exhibited efficient electrochemical performance allowing determination of hydrogen peroxide with low limit of detection of $14 \mathrm{nM}$ and wide linear range of 0.1-380 $\mu \mathrm{M}$. Another $\mathrm{Ti}_{3} \mathrm{C}_{2}$-based electrochemical sensor supplied with haemoglobin to provide direct electron transfer was realized by Liu et al. ${ }^{179}$ These sensor, due to large surface area and high conductivity, displayed good electrochemical performance allowing detection of sodium nitrite $\left(\mathrm{NaNO}_{2}\right)$ with linear range of $0.5-11800 \mu \mathrm{M}$ and limit of detection equal to $0.12 \mu \mathrm{M}$. Rakhi and co-workers have used synergistic effect of Au NPs and MXenes toward glucose oxidation. ${ }^{528}$ Two-dimensional titanium carbide was obtained via exfoliation of $\mathrm{Ti}_{3} \mathrm{AlC}_{2}$. Further sonication of MXene with $\mathrm{Au}$ NPs resulted in $\mathrm{Au} / \mathrm{MXene}$ composite used to prepare electrode together with glucose oxidase (GOx) by casting the obtained mixture onto GCE. These sensors displayed detection of glucose up to $5.9 \mu \mathrm{M}$ and amperometric response with linearity in the range of $0.1 \mathrm{mM}$ to $18 \mathrm{mM}$.

Two-dimensional nanosheets of black phosphorous have gained attention as an analogue of other widely explored layered materials. BP has impressive opto-electronical properties but it is particularly sensible to water and oxygen, thus during the preparation of BP-based chemical sensors particular precautions have to be taken. ${ }^{530,531}$ Yan and co-workers proposed nonenzymatic sensor based on solvent exfoliated BP for determination of $\mathrm{H}_{2} \mathrm{O}_{2} .{ }^{530}$ Fewlayered black phosphorous, produced via a supercritical carbon dioxide-assisted synthesis, were used for electrode preparation by casting ethanolic solution onto GCE. The electrochemical impedance was investigated in $\mathrm{N}_{2}$-saturated phosphate buffered saline (PBS) exhibiting linearity in the range of $1 \times 10^{-7} \mathrm{M}$ to $1 \times 10^{-5} \mathrm{M}$ with limit of detection as low as $1 \times 10^{-7} \mathrm{M}$. Yew et al. used higher affinity of BP NPs toward ssDNA and prepared fluorescent 
sensor for nucleic acid detection. ${ }^{531}$ Biosensing platform provided linearity in the range of 4$4000 \mathrm{pM}$ and limit of detection equal to $5.9 \mathrm{pM}$.

The performance of $\mathrm{hBN}$, MXenes and BP-based materials as (bio)molecular sensors are summarized in Table 11.

Table 11. Comparison of sensing properties of $h B N$, MXenes and BP-based chemical sensors.

\begin{tabular}{|c|c|c|c|c|c|}
\hline Material & Type of sensor & Analyte & $\begin{array}{l}\text { Limit of } \\
\text { detection }\end{array}$ & $\begin{array}{c}\text { Linearity } \\
\text { Range }\end{array}$ & $\operatorname{Ref}$. \\
\hline \multirow{2}{*}{$\begin{array}{c}h B N \\
\text { surfactant exfoliated } \\
h B N\end{array}$} & \multirow[b]{2}{*}{ electrochemical } & $\mathrm{DA}$ & $0.65 \mu \mathrm{M}$ & - & 524 \\
\hline & & & $1.57 \mu \mathrm{M}$ & - & 525 \\
\hline$B N N S$ & fluorescent & DNA & 104 pM & $0.6-20 \mathrm{nM}$ & 526 \\
\hline \multirow{2}{*}{$\begin{array}{c}A g-B N N S \\
A u / B N\end{array}$} & \multirow{2}{*}{ SERS } & R6G & $10 \mu \mathrm{M}$ & - & 168 \\
\hline & & & $1 \mu \mathrm{M}$ & - & 527 \\
\hline \multirow{4}{*}{$\begin{array}{c}\mathrm{TiO}_{2}-\mathrm{Ti}_{3} \mathrm{C}_{2} \\
\mathrm{Ti}_{3} \mathrm{C}_{2} \\
\text { GOx-Au-MXene } \\
\text { BP }\end{array}$} & \multirow{4}{*}{ electrochemical } & $\mathrm{H}_{2} \mathrm{O}_{2}$ & $14 \mathrm{nM}$ & $0.1-380 \mu \mathrm{M}$ & 529 \\
\hline & & $\mathrm{NaNO}_{2}$ & $0.12 \mu \mathrm{M}$ & $0.5-11800 \mu \mathrm{M}$ & 179 \\
\hline & & Glucose & $5.9 \mu \mathrm{M}$ & $0.1-18 \mathrm{mM}$ & 528 \\
\hline & & $\mathrm{H}_{2} \mathrm{O}_{2}$ & $0.1 \mu \mathrm{M}$ & $0.1-10 \mu \mathrm{M}$ & 530 \\
\hline$B P N P S$ & fluorescent & DNA & $5.9 \mathrm{pM}$ & $4-4000 \mathrm{pM}$ & 531 \\
\hline
\end{tabular}

List of abbreviations. DA : dopamine, BNNS : boron nitride nanosheets, SERS : Surface Enhanced Raman Scattering, R6G : rhodamine 6G, GOx : glucose oxidase, BPNPs : black phosphorus nanoparticles.

In this chapter we have discussed 2DMs and 2DM-based composites for the sensing of (bio)molecular species. A range of unique physico-chemical properties offered by 2DMs allows fabrication of various types of chemical sensors including fluorescent, electrochemical, FET and SERS. Recent literature includes investigations of most important biological molecules with particular attention to molecules relevant in medical sciences. Among various advantages of $2 \mathrm{DMs}$ based sensors the extremely low limit of detections (reaching the picomolar level), ultrafast detection $(<1 \mathrm{~s})$ and wide linearity of response signals should be emphasized. Moreover, most of 2DMs based sensors exhibit high selectivity and allow determination of multiple target molecules even in the presence of common interferences. Possibility of further modifications of 2DMs allows achieving multicomponent systems offering better performances in effective determination of target analytes. Noteworthy, the spectrum of molecules detectable with presented sensors is quite narrow. Also there are a few issues that should be considered while development of new sensing 
materials such as stability, reusability and biocompatibility. Electrochemical sensing seems to be one of most widespread approach applied in chemical sensing, which affirms superior electrical properties of graphene and TMD's. In case of TMD recent investigations seem to be mainly focused on the $\mathrm{MoS}_{2}$ and $\mathrm{WS}_{2}$ while the other representatives did not gather much attention so far. Notably the group of novel 2DMs such as hBN, MXenes and BP hold great potential in molecular sensing. Basing on few recent reports those materials constitute promising fundaments for further investigations in terms of its sensing properties.

\section{Conclusions and outlooks}

Alongside the numerous extraordinary physical and chemical properties possessed by 2DMs, their high surface-to-volume ratio represents a key additional advantage for applications in sensing. The all-surface-and-volume-free character guarantees a highest sensitivity to any changes of 2DMs' environment, which is an absolute prerequisite for the development of high-performance sensing devices. However, the use of pristine, defect free sheets may result into weak and unspecific interactions with the chosen analytes. Defects hold a pivotal role in 2DMs: they allow the modulation of the (opto)electronic properties and in particular the engineering of the band gap, which plays a crucial role in improving the sensitivity of the device. Moreover, the presence of dangling groups on the surface can promote analyte absorption, consequently yielding an improvement in the sensing performances. Furthermore, the presence of defects highly increases chemical reactivity of 2DMs enabling further chemical functionalization of the reactive sites, which may result into high specificity, enhanced sensitivity and low detection limit towards targeted analyte. On the other hand, a lack of control during defect engineering can result in irreproducible properties of 2DMs. In particular, the increased reactivity and the presence of dangling bonds with different chemical structures on the basal plane (or edges) of 2DM may result in an enhanced, yet, unselective binding of the analytes ultimately leading in poor selectivity, long response or even incomplete recovery time of the sensor.

One of the greatest challenges to be tackled in the near future is the controlled introduction of ad hoc defects in the 2DMs with a precise spatial resolution and concentration. Ideally, the chemical composition of the defects has to be complemented by a chemical functionalization of the reactive sites with the receptor of the analyte of choice. Such a strategy needs to be pursued by mastering principles of molecular recognitions, e.g. via tailoring of 
supramolecular interactions, which may result not only in ultrasensitive 2DMs but also in superior selectivity and response time - making the actual step towards industrial products. Additionally, chemical programmability, through the generation of multicomponent assemblies based on 2DMs, may enable the future development of multianalyte sensing. Although optical sensors based on the change of the photoluminescent properties of the 2DMs upon interaction with the analytes have been widely and successfully employed for both metals and biologically relevant molecules, electrochemical sensors seem to be the most promising since they provide a direct electric response without the need of employing a transducer. By taking advantage not only of the superior electrical properties of graphene and other 2DMs, but also of their excellent mechanical properties such as robustness and flexibility, 2DMs based sensors can be supported on flexible foils, for smart wearable technologies for monitoring the human/environment interface. Interestingly, the recent demonstration the compatibility of 2DMs with "on-the-skin" applications opens the door towards their use for the detection of clinically relevant biomarkers. Such 2DM-based tattoos may therefore find application as dry on-the-skin sensors characterized by low susceptibility to motion artefacts, which is one of the biggest drawbacks of conventional dry sensors and electrodes for point-of-care and health monitoring.

\section{Acknowledgments}

This work was supported by the European Commission through the Graphene Flagship Core 1 project (GA-696656) and the Polish National Science Centre (Grant no. 2015/18/E/ST5/00188). We also are thankful for the support of the Agence Nationale de la Recherche through the LabEx project Chemistry of Complex Systems (ANR-10-LABX0026_CSC), the International Center for Frontier Research in Chemistry (icFRC). D.P. acknowledges the support from the Embassy of France in Poland in the form of a scholarship at the Institut de Science et d'Ingénierie Supramoléculaires, University of Strasbourg.

\section{Bibliography}

1 K. S. Novoselov, A. K. Geim, S. V. Morozov, D. Jiang, M. I. Katsnelson, I. V. Grigorieva, S. V. Dubonos and A. A. Firsov, Nature, 2005, 438, 197. 
A. C. Ferrari, F. Bonaccorso, V. Fal'ko, K. S. Novoselov, S. Roche, P. Boggild, S. Borini, F. H. L. Koppens, V. Palermo, N. Pugno, J. A. Garrido, R. Sordan, A. Bianco, L. Ballerini, M. Prato, E. Lidorikis, J. Kivioja, C. Marinelli, T. Ryhanen, A. Morpurgo, J. N. Coleman, V. Nicolosi, L. Colombo, A. Fert, M. Garcia-Hernandez, A. Bachtold, G. F. Schneider, F. Guinea, C. Dekker, M. Barbone, Z. Sun, C. Galiotis, A. N. Grigorenko, G. Konstantatos, A. Kis, M. Katsnelson, L. Vandersypen, A. Loiseau, V. Morandi, D. Neumaier, E. Treossi, V. Pellegrini, M. Polini, A. Tredicucci, G. M. Williams, B. Hee Hong, J.-H. Ahn, J. Min Kim, H. Zirath, B. J. van Wees, H. van der Zant, L. Occhipinti, A. Di Matteo, I. A. Kinloch, T. Seyller, E. Quesnel, X. Feng, K. Teo, N. Rupesinghe, P. Hakonen, S. R. T. Neil, Q. Tannock, T. Lofwander and J. Kinaret, Nanoscale, 2015, 7, 4598.

Q. H. Wang, K. Kalantar-Zadeh, A. Kis, J. N. Coleman and M. S. Strano, Nat. Nanotechnol., 2012, 7, 699.

$4 \quad$ M. W. Barsoum, Prog. Solid State Chem., 2000, 28, 201.

5 L. Li, Y. Yu, G. J. Ye, Q. Ge, X. Ou, H. Wu, D. Feng, X. H. Chen and Y. Zhang, Nat. Nanotechnol., 2014, 9, 372.

6 S. P. Koenig, R. A. Doganov, H. Schmidt, A. H. Castro Neto and B. Özyilmaz, Appl. Phys. Lett., 2014, 104, 103106

7 H. Liu, A. T. Neal, Z. Zhu, Z. Luo, X. Xu, D. Tománek and P. D. Ye, ACS Nano, $2014,8,4033$.

8 A. Gupta, T. Sakthivel and S. Seal, Prog. Mater. Sci., 2015, 73, 44.

9 A. S. Mayorov, R. V. Gorbachev, S. V. Morozov, L. Britnell, R. Jalil, L. A. Ponomarenko, P. Blake, K. S. Novoselov, K. Watanabe, T. Taniguchi and A. K. Geim, Nano Lett., 2011, 11, 2396.

10 D. C. Elias, R. V. Gorbachev, A. S. Mayorov, S. V. Morozov, A. A. Zhukov, P. Blake, L. A. Ponomarenko, I. V. Grigorieva, K. S. Novoselov, F. Guinea and A. K. Geim, Nat. Phys., 2011, 7, 701.

11 A. Kuc, N. Zibouche and T. Heine, Phys. Rev. B, 2011, 83, 245213.

12 A. Splendiani, L. Sun, Y. Zhang, T. Li, J. Kim, C.-Y. Chim, G. Galli and F. Wang, Nano Lett., 2010, 10, 1271.

13 J.-S. Kim, H.-W. Yoo, H. O. Choi and H.-T. Jung, Nano Lett., 2014, 14, 5941.

14 D. J. Late, Y.-K. Huang, B. Liu, J. Acharya, S. N. Shirodkar, J. Luo, A. Yan, D. Charles, U. V. Waghmare, V. P. Dravid and C. N. R. Rao, ACS Nano, 2013, 7, 4879.

15 K. Kalantar-zadeh and J. Z. Ou, ACS Sens., 2016, 1, 5.

16 Y. Song, Y. Luo, C. Zhu, H. Li, D. Du and Y. Lin, Biosens. Bioelectron., 2016, 76, 195.

17 V. Georgakilas, M. Otyepka, A. B. Bourlinos, V. Chandra, N. Kim, K. C. Kemp, P. Hobza, R. Zboril and K. S. Kim, Chem. Rev., 2012, 112, 6156.

18 B. Cai, S. Zhang, Z. Yan and H. Zeng, ChemNanoMat, 2015, 1, 542.

19 E. A. Meyer, R. K. Castellano and F. Diederich, Angew. Chem. Int. Ed., 2003, 42, 1210.

20 S. K. Burley and G. A. Petsko, FEBS Lett., 1986, 203, 139.

21 P. Tarakeshwar, K. S. Kim, E. Kraka and D. Cremer, J. Chem. Phys., 2001, 115, 6018.

22 E. C. Lee, B. H. Hong, J. Y. Lee, J. C. Kim, D. Kim, Y. Kim, P. Tarakeshwar and K. S. Kim, J. Am. Chem. Soc., 2005, 127, 4530.

23 S. J. Grabowski, J. Phys. Chem. A, 2001, 105, 10739.

24 P. Tarakeshwar, H. S. Choi and K. S. Kim, J. Am. Chem. Soc., 2001, 123, 3323.

25 E.-i. Kim, S. Paliwal and C. S. Wilcox, J. Am. Chem. Soc., 1998, 120, 11192.

26 K. E. Riley, M. Pitoňák, P. Jurečka and P. Hobza, Chem. Rev., 2010, 110, 5023. 
29 E. C. Lee, D. Kim, P. Jurečka, P. Tarakeshwar, P. Hobza and K. S. Kim, J. Phys. Chem. A, 2007, 111, 3446.

30 I. Geronimo, E. C. Lee, N. J. Singh and K. S. Kim, J. Chem. Theory Comput., 2010, 6, 1931.

31 W. Wang and P. Hobza, ChemPhysChem, 2008, 9, 1003.

32 D. A. Dougherty and D. A. Stauffer, Science, 1990, 250, 1558.

33 D. Kim, S. Hu, P. Tarakeshwar, K. S. Kim and J. M. Lisy, J. Phys. Chem. A, 2003, 107, 1228 .

34 S. Tsuzuki, M. Yoshida, T. Uchimaru and M. Mikami, J. Phys. Chem. A, 2001, 105, 769.

35 S. E. Wheeler and K. N. Houk, J. Am. Chem. Soc., 2009, 131, 3126.

36 M. A. Gebbie, W. Wei, A. M. Schrader, T. R. Cristiani, H. A. Dobbs, M. Idso, B. F. Chmelka, J. H. Waite and J. N. Israelachvili, Nat. Chem., 2017, 9, 473.

37 A. S. Mahadevi and G. N. Sastry, Chem. Rev., 2013, 113, 2100.

38 P. B. Crowley and A. Golovin, Proteins: Struct., Funct., Bioinf., 2005, 59, 231.

39 J. P. Gallivan and D. A. Dougherty, Proc. Natl. Acad. Sci. U.S.A., 1999, 96, 9459.

$40 \quad$ K. S. Kim, P. Tarakeshwar and J. Y. Lee, Chem. Rev., 2000, 100, 4145.

41 D. Quiñonero, C. Garau, C. Rotger, A. Frontera, P. Ballester, A. Costa and P. M. Deyà, Angew. Chem. Int. Ed., 2002, 41, 3389.

42 B. L. Schottel, H. T. Chifotides and K. R. Dunbar, Chem. Soc. Rev., 2008, 37, 68.

43 Y. S. Rosokha, S. V. Lindeman, S. V. Rosokha and J. K. Kochi, Angew. Chem. Int. Ed., 2004, 43, 4650.

44 P. De Hoog, P. Gamez, I. Mutikainen, U. Turpeinen and J. Reedijk, Angew. Chem. Int. Ed., 2004, 116, 5815.

45 R. E. Dawson, A. Hennig, D. P. Weimann, D. Emery, V. Ravikumar, J. Montenegro, T. Takeuchi, S. Gabutti, M. Mayor, J. Mareda, C. A. Schalley and S. Matile, Nat. Chem., 2010, 2, 533.

46 A. Frontera, P. Gamez, M. Mascal, T. J. Mooibroek and J. Reedijk, Angew. Chem. Int. Ed., 2011, 50, 9564.

47 B. L. Schottel, H. T. Chifotides, M. Shatruk, A. Chouai, L. M. Pérez, J. Bacsa and K. R. Dunbar, J. Am. Chem. Soc., 2006, 128, 5895.

48 H. T. Chifotides and K. R. Dunbar, Acc. Chem. Res., 2013, 46, 894.

49 C. Garau, A. Frontera, D. Quiñonero, P. Ballester, A. Costa and P. M. Deyà, J. Phys. Chem. A, 2004, 108, 9423.

50 D. Kim, P. Tarakeshwar and K. S. Kim, J. Phys. Chem. A, 2004, 108, 1250.

51 P. G. Moses, J. J. Mortensen, B. I. Lundqvist and J. K. Nørskov, J. Chem. Phys., 2009, 130, 104709.

52 N. Huo, S. Yang, Z. Wei, S. S. Li, J. B. Xia and J. Li, Sci. Rep., 2014, 4, 5209.

53 A. Hashimoto, K. Suenaga, A. Gloter, K. Urita and S. Iijima, Nature, 2004, 430, 870.

54 W. Zhou, X. Zou, S. Najmaei, Z. Liu, Y. Shi, J. Kong, J. Lou, P. M. Ajayan, B. I. Yakobson and J.-C. Idrobo, Nano Lett., 2013, 13, 2615.

55 F. Banhart, J. Kotakoski and A. V. Krasheninnikov, ACS Nano, 2011, 5, 26.

56 L. G. Cançado, A. Jorio, E. H. M. Ferreira, F. Stavale, C. A. Achete, R. B. Capaz, M. V. O. Moutinho, A. Lombardo, T. S. Kulmala and A. C. Ferrari, Nano Lett., 2011, 11, 3190.

57 S. Feng, Z. Lin, X. Gan, R. Lv and M. Terrones, Nanoscale Horiz., 2017, 2, 72.

58 A. Ambrosi and M. Pumera, Chem. - Eur. J., 2010, 16, 10946. 
59 K. C. Santosh, C. L. Roberto, A. Rafik, M. W. Robert and C. Kyeongjae, Nanotechnology, 2014, 25, 375703.

60 D. Liu, Y. Guo, L. Fang and J. Robertson, Appl. Phys. Lett., 2013, 103, 183113

61 S. Tongay, J. Suh, C. Ataca, W. Fan, A. Luce, J. S. Kang, J. Liu, C. Ko, R. Raghunathanan, J. Zhou, F. Ogletree, J. Li, J. C. Grossman and J. Wu, Sci. Rep., 2013, 3, 2657.

62 H. Nan, Z. Wang, W. Wang, Z. Liang, Y. Lu, Q. Chen, D. He, P. Tan, F. Miao, X. Wang, J. Wang and Z. Ni, ACS Nano, 2014, 8, 5738.

63 J. Xie, H. Zhang, S. Li, R. Wang, X. Sun, M. Zhou, J. Zhou, X. W. Lou and Y. Xie, Adv. Mater., 2013, 25, 5807.

64 L. Rodriguez-Perez, M. a. A. Herranz and N. Martin, Chem. Commun., 2013, 49, 3721.

65 G. L. C. Paulus, Q. H. Wang and M. S. Strano, Acc. Chem. Res., 2013, 46, 160.

66 S. Niyogi, E. Bekyarova, M. E. Itkis, H. Zhang, K. Shepperd, J. Hicks, M. Sprinkle, C. Berger, C. N. Lau, W. A. de Heer, E. H. Conrad and R. C. Haddon, Nano Lett., 2010, 10, 4061.

67 J. M. Englert, C. Dotzer, G. Yang, M. Schmid, C. Papp, J. M. Gottfried, H.-P. Steinrück, E. Spiecker, F. Hauke and A. Hirsch, Nat. Chem., 2011, 3, 279.

68 H. Liu, S. Ryu, Z. Chen, M. L. Steigerwald, C. Nuckolls and L. E. Brus, J. Am. Chem. Soc., 2009, 131, 17099.

69 M. Quintana, K. Spyrou, M. Grzelczak, W. R. Browne, P. Rudolf and M. Prato, ACS Nano, 2010, 4, 3527.

70 D. Chen, H. Feng and J. Li, Chem. Rev., 2012, $112,6027$.

71 D. R. Dreyer, S. Park, C. W. Bielawski and R. S. Ruoff, Chem. Soc. Rev., 2010, 39, 228.

72 M. Zhou, Y. Zhai and S. Dong, Anal. Chem., 2009, 81, 5603.

73 J. T. Robinson, F. K. Perkins, E. S. Snow, Z. Wei and P. E. Sheehan, Nano Lett., $2008,8,3137$.

74 S. Wu, Q. He, C. Tan, Y. Wang and H. Zhang, Small, 2013, 9, 1160.

75 W. Yuan and G. Shi, J. Mater. Chem. A, 2013, 1, 10078.

76 Q. He, S. Wu, Z. Yin and H. Zhang, Chem. Sci., 2012, 3, 1764.

77 G. Zhao, J. Li, X. Ren, C. Chen and X. Wang, Environ. Sci. Technol., 2011, 45, 10454.

78 R. Sitko, E. Turek, B. Zawisza, E. Malicka, E. Talik, J. Heimann, A. Gagor, B. Feist and R. Wrzalik, Dalton Trans., 2013, 42, 5682.

79 G. Eda and M. Chhowalla, Adv. Mater., 2010, 22, 2392.

80 G. Ko, H. Y. Kim, J. Ahn, Y. M. Park, K. Y. Lee and J. Kim, Curr. Appl. Phys., 2010, 10, 1002.

81 D. R. Dreyer, A. D. Todd and C. W. Bielawski, Chem. Soc. Rev., 2014, 43, 5288.

82 S. Eigler and A. Hirsch, Angew. Chem. Int. Ed., 2014, 53, 7720.

83 C. K. Chua and M. Pumera, Chem. Soc. Rev., 2014, 43, 291.

84 L. Zhou, B. He, Y. Yang and Y. He, RSC Adv., 2014, 4, 32570.

85 C. Tsai, H. Li, S. Park, J. Park, H. S. Han, J. K. Nørskov, X. Zheng and F. AbildPedersen, Nat. Commun., 2017, 8, 15113.

86 S. Bertolazzi, S. Bonacchi, G. Nan, A. Pershin, D. Beljonne and P. Samorì, Adv. Mater., 2017, 29, 1606760

87 K. Cho, M. Min, T.-Y. Kim, H. Jeong, J. Pak, J.-K. Kim, J. Jang, S. J. Yun, Y. H. Lee, W.-K. Hong and T. Lee, ACS Nano, 2015, 9, 8044. 

J. S. Spencer, S. Zhuiykov, K. Kalantar-zadeh and T. Daeneke, Adv. Mater., 2015, 27, 6225.

89 A. Aliprandi, D. Pakulski, A. Ciesielski and P. Samorì, ACS Nano, 2017, 11, 10654.

90 B. M. Venkatesan and R. Bashir, Nat. Nanotechnol., 2011, 6, 615.

91 S. Garaj, W. Hubbard, A. Reina, J. Kong, D. Branton and J. A. Golovchenko, Nature, 2010, 467, 190.

92 S. Mao, J. Chang, H. Pu, G. Lu, Q. He, H. Zhang and J. Chen, Chem. Soc. Rev., 2017, 46, 6872 .

93 Y. Liu, X. Dong and P. Chen, Chem. Soc. Rev., 2012, 41, 2283.

94 D. Chen, L. Tang and J. Li, Chem. Soc. Rev., 2010, 39, 3157.

95 M. J. Allen, V. C. Tung and R. B. Kaner, Chem. Rev., 2010, 110, 132.

96 S. Cui, H. Pu, S. A. Wells, Z. Wen, S. Mao, J. Chang, M. C. Hersam and J. Chen, Nat. Commun., 2015, 6, 8632.

97 Y. Wang, Z. Li, J. Wang, J. Li and Y. Lin, Trends Biotechnol., 2011, 29, 205.

98 Z. Tang, H. Wu, J. R. Cort, G. W. Buchko, Y. Zhang, Y. Shao, I. A. Aksay, J. Liu and Y. Lin, Small, 2010, 6, 1205.

99 C. Zhu, Z. Zeng, H. Li, F. Li, C. Fan and H. Zhang, J. Am. Chem. Soc., 2013, 135, 5998.

100 D. Dinda, A. Gupta, B. K. Shaw, S. Sadhu and S. K. Saha, ACS Appl. Mater. Interfaces, 2014, 6, 10722.

101 X. Ling, L. Xie, Y. Fang, H. Xu, H. Zhang, J. Kong, M. S. Dresselhaus, J. Zhang and Z. Liu, Nano Lett., 2010, 10, 553.

102 X. Ling, W. Fang, Y.-H. Lee, P. T. Araujo, X. Zhang, J. F. Rodriguez-Nieva, Y. Lin, J. Zhang, J. Kong and M. S. Dresselhaus, Nano Lett., 2014, 14, 3033.

103 S. Schlücker, Angew. Chem. Int. Ed., 2014, 53, 4756.

104 S. Laing, L. E. Jamieson, K. Faulds and D. Graham, Nat. Rev. Chem., 2017, 1, 0060.

105 A.-I. Henry, B. Sharma, M. F. Cardinal, D. Kurouski and R. P. Van Duyne, Anal. Chem., 2016, 88, 6638.

106 M. F. Cardinal, E. Vander Ende, R. A. Hackler, M. O. McAnally, P. C. Stair, G. C. Schatz and R. P. Van Duyne, Chem. Soc. Rev., 2017, 46, 3886.

107 S. Nie and S. R. Emory, Science, 1997, 275, 1102.

108 K. Kneipp, Y. Wang, H. Kneipp, L. T. Perelman, I. Itzkan, R. R. Dasari and M. S. Feld, Phys. Rev. Lett., 1997, 78, 1667.

109 L. Sun, H. Hu, D. Zhan, J. Yan, L. Liu, J. S. Teguh, E. K. L. Yeow, P. S. Lee and Z. Shen, Small, 2014, 10, 1090.

110 X. Ling, S. Huang, S. Deng, N. Mao, J. Kong, M. S. Dresselhaus and J. Zhang, Acc. Chem. Res., 2015, 48, 1862.

111 A. J. Bandodkar, I. Jeerapan and J. Wang, ACS Sens., 2016, 1, 464.

112 E. Singh, M. Meyyappan and H. S. Nalwa, ACS Appl. Mater. Interfaces, 2017, 9, 34544.

113 H. Lee, T. K. Choi, Y. B. Lee, H. R. Cho, R. Ghaffari, L. Wang, H. J. Choi, T. D. Chung, N. Lu, T. Hyeon, S. H. Choi and D.-H. Kim, Nat. Nanotechnol., 2016, 11, 566.

114 N. O. Weiss, H. Zhou, L. Liao, Y. Liu, S. Jiang, Y. Huang and X. Duan, Adv. Mater., 2012, 24, 5782.

115 V. Berry, Carbon, 2013, 62, 1.

116 F. Guo, G. Silverberg, S. Bowers, S.-P. Kim, D. Datta, V. Shenoy and R. H. Hurt, Environ. Sci. Technol., 2012, 46, 7717. 
117 D. Pierleoni, Z. Y. Xia, M. Christian, S. Ligi, M. Minelli, V. Morandi, F. Doghieri and V. Palermo, Carbon, 2016, 96, 503.

118 D. Prasai, J. C. Tuberquia, R. R. Harl, G. K. Jennings and K. I. Bolotin, ACS Nano, 2012, 6, 1102 .

119 A. K. Geim, Science, 2009, 324, 1530.

120 X. Li, W. Cai, J. An, S. Kim, J. Nah, D. Yang, R. Piner, A. Velamakanni, I. Jung, E. Tutuc, S. K. Banerjee, L. Colombo and R. S. Ruoff, Science, 2009, 324, 1312.

121 Y. Zhang, L. Zhang and C. Zhou, Acc. Chem. Res., 2013, 46, 2329.

122 C. Berger, Z. M. Song, T. B. Li, X. B. Li, A. Y. Ogbazghi, R. Feng, Z. T. Dai, A. N. Marchenkov, E. H. Conrad, P. N. First and W. A. de Heer, J. Phys. Chem. B, 2004, 108, 19912.

123 Y. Zhu, S. Murali, W. Cai, X. Li, J. W. Suk, J. R. Potts and R. S. Ruoff, Adv. Mater., 2010, 22, 3906.

124 S. Basu and P. Bhattacharyya, Sens. Actuators, B, 2012, 173, 1.

125 Y. Hernandez, V. Nicolosi, M. Lotya, F. M. Blighe, Z. Sun, S. De, I. T. McGovern, B. Holland, M. Byrne, Y. K. Gun'Ko, J. J. Boland, P. Niraj, G. S. Duesberg, S. Krishnamurthy, R. Goodhue, J. Hutchison, V. Scardaci, A. C. Ferrari and J. N. Coleman, Nat. Nanotechnol., 2008, 3, 563.

126 A. Ciesielski and P. Samorì, Adv. Mater., 2016, 28, 6030.

127 K. R. Paton, E. Varrla, C. Backes, R. J. Smith, U. Khan, A. O’Neill, C. Boland, M. Lotya, O. M. Istrate, P. King, T. Higgins, S. Barwich, P. May, P. Puczkarski, I. Ahmed, M. Moebius, H. Pettersson, E. Long, J. Coelho, S. E. O'Brien, E. K. McGuire, B. M. Sanchez, G. S. Duesberg, N. McEvoy, T. J. Pennycook, C. Downing, A. Crossley, V. Nicolosi and J. N. Coleman, Nat. Mater., 2014, 13, 624.

128 M. Eredia, S. Bertolazzi, T. Leydecker, M. El Garah, I. Janica, G. Melinte, O. Ersen, A. Ciesielski and P. Samorì, J. Phys. Chem. Lett., 2017, 8, 3347.

129 Z. Yin, H. Li, H. Li, L. Jiang, Y. Shi, Y. Sun, G. Lu, Q. Zhang, X. Chen and H. Zhang, ACS Nano, 2012, 6, 74.

130 W. Wu, L. Wang, Y. Li, F. Zhang, L. Lin, S. Niu, D. Chenet, X. Zhang, Y. Hao, T. F. Heinz, J. Hone and Z. L. Wang, Nature, 2014, 514, 470.

131 Y. Sun, S. Gao and Y. Xie, Chem. Soc. Rev., 2014, 43, 530.

132 G. Zhang, G. Proni, S. Zhao, E. C. Constable, C. E. Housecroft, M. Neuburger and J. A. Zampese, Dalton Trans., 2014, 43, 12313.

133 Kenry, A. Geldert, X. Zhang, H. Zhang and C. T. Lim, ACS Sens., 2016, 1, 1315.

134 T. Yang, R. Yang, H. Chen, F. Nan, T. Ge and K. Jiao, ACS Appl. Mater. Interfaces, 2015, 7, 2867.

135 M. Chhowalla, H. S. Shin, G. Eda, L.-J. Li, K. P. Loh and H. Zhang, Nat. Chem., 2013, 5, 263.

136 Y. Li, K.-A. N. Duerloo, K. Wauson and E. J. Reed, Nat. Commun., 2016, 7, 10671.

137 J. N. Coleman, M. Lotya, A. O’Neill, S. D. Bergin, P. J. King, U. Khan, K. Young, A. Gaucher, S. De, R. J. Smith, I. V. Shvets, S. K. Arora, G. Stanton, H.-Y. Kim, K. Lee, G. T. Kim, G. S. Duesberg, T. Hallam, J. J. Boland, J. J. Wang, J. F. Donegan, J. C. Grunlan, G. Moriarty, A. Shmeliov, R. J. Nicholls, J. M. Perkins, E. M. Grieveson, K. Theuwissen, D. W. McComb, P. D. Nellist and V. Nicolosi, Science, 2011, 331, 568.

138 D. Kong, H. Wang, J. J. Cha, M. Pasta, K. J. Koski, J. Yao and Y. Cui, Nano Lett., 2013, 13, 1341.

139 J. Shi, D. Ma, G.-F. Han, Y. Zhang, Q. Ji, T. Gao, J. Sun, X. Song, C. Li, Y. Zhang, X.-Y. Lang, Y. Zhang and Z. Liu, ACS Nano, 2014, 8, 10196.

140 H. S. S. Ramakrishna Matte, A. Gomathi, A. K. Manna, D. J. Late, R. Datta, S. K. Pati and C. N. R. Rao, Angew. Chem. Int. Ed., 2010, 49, 4059. 
141 Z. Zeng, Z. Yin, X. Huang, H. Li, Q. He, G. Lu, F. Boey and H. Zhang, Angew. Chem. Int. Ed., 2011, 50, 11093.

142 V. Nicolosi, M. Chhowalla, M. G. Kanatzidis, M. S. Strano and J. N. Coleman, Science, 2013, 340, 1226419.

143 Y.-H. Lee, X.-Q. Zhang, W. Zhang, M.-T. Chang, C.-T. Lin, K.-D. Chang, Y.-C. Yu, J. T.-W. Wang, C.-S. Chang, L.-J. Li and T.-W. Lin, Adv. Mater., 2012, 24, 2320.

144 B. Liu, L. Chen, G. Liu, A. N. Abbas, M. Fathi and C. Zhou, ACS Nano, 2014, 8, 5304.

145 K. Lee, R. Gatensby, N. McEvoy, T. Hallam and G. S. Duesberg, Adv Mater, 2013, 25, 6699 .

146 B. Cho, A. R. Kim, Y. Park, J. Yoon, Y. J. Lee, S. Lee, T. J. Yoo, C. G. Kang, B. H. Lee, H. C. Ko, D. H. Kim and M. G. Hahm, ACS Appl. Mater. Interfaces, 2015, 7, 2952.

147 S.-Y. Cho, S. J. Kim, Y. Lee, J.-S. Kim, W.-B. Jung, H.-W. Yoo, J. Kim and H.-T. Jung, ACS Nano, 2015, 9, 9314.

148 K. F. Mak, C. Lee, J. Hone, J. Shan and T. F. Heinz, Phys. Rev. Lett., 2010, 105, 136805.

149 T. Wu and H. Zhang, Angew. Chem. Int. Ed., 2015, 54, 4432.

150 D. M. Sim, H. J. Han, S. Yim, M.-J. Choi, J. Jeon and Y. S. Jung, ACS Omega, 2017, 2, 4678 .

151 C. H. Ho and C. E. Huang, J. Alloys Compd., 2004, 383, 74.

152 Y. Wang, L. Li, W. Yao, S. Song, J. T. Sun, J. Pan, X. Ren, C. Li, E. Okunishi, Y.-Q. Wang, E. Wang, Y. Shao, Y. Y. Zhang, H.-t. Yang, E. F. Schwier, H. Iwasawa, K. Shimada, M. Taniguchi, Z. Cheng, S. Zhou, S. Du, S. J. Pennycook, S. T. Pantelides and H.-J. Gao, Nano Lett., 2015, 15, 4013.

153 A. Carvalho, M. Wang, X. Zhu, A. S. Rodin, H. Su and A. H. Castro Neto, Nat. Rev. Mater., 2016, 1, 16061.

154 A. Castellanos-Gomez, L. Vicarelli, E. Prada, J. O. Island, K. L. Narasimha-Acharya, S. I. Blanter, D. J. Groenendijk, M. Buscema, G. A. Steele, J. V. Alvarez, H. W. Zandbergen, J. J. Palacios and H. S. J. van der Zant, 2D Mater., 2014, 1, 25001.

155 M. Buscema, D. J. Groenendijk, G. A. Steele, H. S. J. van der Zant and A. Castellanos-Gomez, Nat. Commun., 2014, 5, 4651.

156 J. Qiao, X. Kong, Z.-X. Hu, F. Yang and W. Ji, Nat. Commun., 2014, 5, 4475.

157 C. Backes, R. J. Smith, N. McEvoy, N. C. Berner, D. McCloskey, H. C. Nerl, A. O’Neill, P. J. King, T. Higgins, D. Hanlon, N. Scheuschner, J. Maultzsch, L. Houben, G. S. Duesberg, J. F. Donegan, V. Nicolosi and J. N. Coleman, Nat. Commun., 2014, 5, 4576.

158 A. N. Abbas, B. Liu, L. Chen, Y. Ma, S. Cong, N. Aroonyadet, M. Köpf, T. Nilges and C. Zhou, ACS Nano, 2015, 9, 5618.

159 Y. Cai, Q. Ke, G. Zhang and Y.-W. Zhang, J. Phys. Chem. C, 2015, 119, 3102.

160 C. C. Mayorga-Martinez, Z. Sofer and M. Pumera, Angew. Chem. Int. Ed., 2015, 127, 14317.

161 L. Kou, T. Frauenheim and C. Chen, J. Phys. Chem. Lett., 2014, 5, 2675.

162 J. O. Island, G. A. Steele, H. S. J. van der Zant and A. Castellanos-Gomez, 2D Mater., 2015, 2, 11002.

163 V. L. Solozhenko, A. G. Lazarenko, J. P. Petitet and A. V. Kanaev, J. Phys. Chem. Solids, 2001, 62, 1331.

164 K. Watanabe, T. Taniguchi and H. Kanda, Nat. Mater., 2004, 3, 404.

165 A. Nag, K. Raidongia, K. P. S. S. Hembram, R. Datta, U. V. Waghmare and C. N. R. Rao, ACS Nano, 2010, 4, 1539. 
166 L. Liu, Y. P. Feng and Z. X. Shen, Phys. Rev. B, 2003, 68, 104102.

167 D. Pacilé, J. C. Meyer, Ç. Ö. Girit and A. Zettl, Appl. Phys. Lett., 2008, 92, 133107.

168 Y. Lin, C. E. Bunker, K. A. S. Fernando and J. W. Connell, ACS Appl. Mater. Interfaces, 2012, 4, 1110.

169 W.-Q. Han, L. Wu, Y. Zhu, K. Watanabe and T. Taniguchi, Appl. Phys. Lett., 2008, 93, 223103.

170 C. Zhi, Y. Bando, C. Tang, H. Kuwahara and D. Golberg, Adv. Mater., 2009, 21, 2889.

171 L. Cao, S. Emami and K. Lafdi, Mater. Express, 2014, 4, 165.

172 F. Müller, K. Stöwe and H. Sachdev, Chem. Mater., 2005, 17, 3464.

173 W. Auwärter, H. U. Suter, H. Sachdev and T. Greber, Chem. Mater., 2004, 16, 343.

174 Y.-H. Lee, K.-K. Liu, A.-Y. Lu, C.-Y. Wu, C.-T. Lin, W. Zhang, C.-Y. Su, C.-L. Hsu, T.-W. Lin, K.-H. Wei, Y. Shi and L.-J. Li, RSC $A d v$., 2012, 2, 111.

175 M. Naguib, O. Mashtalir, J. Carle, V. Presser, J. Lu, L. Hultman, Y. Gogotsi and M. W. Barsoum, ACS Nano, 2012, 6, 1322.

176 O. Mashtalir, M. Naguib, V. N. Mochalin, Y. Dall'Agnese, M. Heon, M. W. Barsoum and Y. Gogotsi, Nat. Commun., 2013, 4, 1716.

177 M. R. Lukatskaya, O. Mashtalir, C. E. Ren, Y. Dall'Agnese, P. Rozier, P. L. Taberna, M. Naguib, P. Simon, M. W. Barsoum and Y. Gogotsi, Science, 2013, 341, 1502.

178 L. Lorencova, T. Bertok, E. Dosekova, A. Holazova, D. Paprckova, A. Vikartovska, V. Sasinkova, J. Filip, P. Kasak, M. Jerigova, D. Velic, K. A. Mahmoud and J. Tkac, Electrochim. Acta, 2017, 235, 471.

179 H. Liu, C. Duan, C. Yang, W. Shen, F. Wang and Z. Zhu, Sens. Actuators, B, 2015, 218, 60 .

180 S. Yang, C. Jiang and S.-H. Wei, Appl. Phys. Rev., 2017, 4, 21304

181 G. F. Fine, L. M. Cavanagh, A. Afonja and R. Binions, Sensors, 2010, 10, 5469.

182 Y. Pak, S.-M. Kim, H. Jeong, C. G. Kang, J. S. Park, H. Song, R. Lee, N. Myoung, B. H. Lee, S. Seo, J. T. Kim and G.-Y. Jung, ACS Appl. Mater. Interfaces, 2014, 6, 13293.

183 J. Zhang, X. Liu, G. Neri and N. Pinna, Adv. Mater., 2016, 28, 795.

184 S. J. Choi, B. H. Jang, S. J. Lee, B. K. Min, A. Rothschild and I. D. Kim, ACS Appl. Mater. Interfaces, 2014, 6, 2588.

185 W. Li, X. Geng, Y. Guo, J. Rong, Y. Gong, L. Wu, X. Zhang, P. Li, J. Xu, G. Cheng, M. Sun and L. Liu, ACS Nano, 2011, 5, 6955.

186 C. Wang, L. Yin, L. Zhang, D. Xiang and R. Gao, Sensors, 2010, 10, 2088.

187 S. A. Waghuley, S. M. Yenorkar, S. S. Yawale and S. P. Yawale, Sens. Actuators, B, 2008, 128, 366.

188 J. Li, Y. Lu, Q. Ye, M. Cinke, J. Han and M. Meyyappan, Nano Lett., 2003, 3, 929.

189 A. K. Geim and K. S. Novoselov, Nat. Mater., 2007, 6, 183.

190 H. Li, Z. Yin, Q. He, H. Li, X. Huang, G. Lu, D. W. Fam, A. I. Tok, Q. Zhang and H. Zhang, Small, 2012, 8, 63.

191 Q. Yue, Z. Shao, S. Chang and J. Li, Nanoscale Res. Lett., 2013, 8, 425.

192 M. Donarelli, S. Prezioso, F. Perrozzi, F. Bisti, M. Nardone, L. Giancaterini, C. Cantalini and L. Ottaviano, Sens. Actuators, B, 2015, 207, 602.

193 F. K. Perkins, A. L. Friedman, E. Cobas, P. M. Campbell, G. G. Jernigan and B. T. Jonker, Nano Lett., 2013, 13, 668.

194 M. O’Brien, K. Lee, R. Morrish, N. C. Berner, N. McEvoy, C. A. Wolden and G. S. Duesberg, Chem. Phys. Lett., 2014, 615, 6.

195 C. C. Mayorga-Martinez, A. Ambrosi, A. Y. S. Eng, Z. Sofer and M. Pumera, Adv. Funct. Mater., 2015, 25, 5611. 
196 M. Ferroni, V. Guidi, G. Martinelli, P. Nelli, M. Sacerdoti and G. Sberveglieri, Thin Solid Films, 1997, 307, 148.

197 K. Xu, N. Li, D. Zeng, S. Tian, S. Zhang, D. Hu and C. Xie, ACS Appl. Mater. Interfaces, 2015, 7, 11359.

198 D. Zhang, Y. Sun, P. Li and Y. Zhang, ACS Appl. Mater. Interfaces, 2016, 8, 14142.

199 M. Sajjad, G. Morell and P. Feng, ACS Appl. Mater. Interfaces, 2013, 5, 5051.

200 N. L. Teradal, S. Marx, A. Morag and R. Jelinek, J. Mater. Chem. C, 2017, 5, 1128.

201 F. Schedin, A. K. Geim, S. V. Morozov, E. W. Hill, P. Blake, M. I. Katsnelson and K. S. Novoselov, Nat. Mater., 2007, 6, 652.

202 X. Huang, X. Y. Qi, F. Boey and H. Zhang, Chem. Soc. Rev., 2012, 41, 666.

203 O. Leenaerts, B. Partoens and F. M. Peeters, Phys. Rev. B, 2008, 77, 125416.

204 Y. Dan, Y. Lu, N. J. Kybert, Z. Luo and A. T. C. Johnson, Nano Lett., 2009, 9, 1472.

205 S. Rumyantsev, G. Liu, M. S. Shur, R. A. Potyrailo and A. A. Balandin, Nano Lett., 2012, 12, 2294.

206 H. J. Yoon, D. H. Jun, J. H. Yang, Z. Zhou, S. S. Yang and M. M.-C. Cheng, Sens. Actuators, B, 2011, 157, 310 .

207 S. Kumar, S. Kaushik, R. Pratap and S. Raghavan, ACS Appl. Mater. Interfaces, 2015, 7, 2189.

208 M. G. Chung, D. H. Kim, H. M. Lee, T. Kim, J. H. Choi, D. k. Seo, J.-B. Yoo, S.-H. Hong, T. J. Kang and Y. H. Kim, Sens. Actuators, B, 2012, 166-167, 172.

209 H. Choi, J. S. Choi, J. S. Kim, J. H. Choe, K. H. Chung, J. W. Shin, J. T. Kim, D. H. Youn, K. C. Kim, J. I. Lee, S. Y. Choi, P. Kim, C. G. Choi and Y. J. Yu, Small, 2014, 10, 3685 .

210 Y. H. Kim, S. J. Kim, Y.-J. Kim, Y.-S. Shim, S. Y. Kim, B. H. Hong and H. W. Jang, ACS Nano, 2015, 9, 10453.

211 M. Gautam and A. H. Jayatissa, Mater. Sci. Eng., C, 2011, 31, 1405.

212 F. Yavari, Z. Chen, A. V. Thomas, W. Ren, H.-M. Cheng and N. Koratkar, Sci. Rep., $2011,1,166$.

213 A. D. Smith, K. Elgammal, X. Fan, M. C. Lemme, A. Delin, M. Råsander, L. Bergqvist, S. Schröder, A. C. Fischer, F. Niklaus and M. Östling, RSC Adv., 2017, 7, 22329.

214 M. W. K. Nomani, R. Shishir, M. Qazi, D. Diwan, V. B. Shields, M. G. Spencer, G. S. Tompa, N. M. Sbrockey and G. Koley, Sens. Actuators, B, 2010, 150, 301.

215 F. Varchon, R. Feng, J. Hass, X. Li, B. N. Nguyen, C. Naud, P. Mallet, J. Y. Veuillen, C. Berger, E. H. Conrad and L. Magaud, Phys. Rev. Lett., 2007, 99, 126805.

216 R. Pearce, T. Iakimov, M. Andersson, L. Hultman, A. L. Spetz and R. Yakimova, Sens. Actuators, B, 2011, 155, 451.

217 Y. R. Choi, Y.-G. Yoon, K. S. Choi, J. H. Kang, Y.-S. Shim, Y. H. Kim, H. J. Chang, J.-H. Lee, C. R. Park, S. Y. Kim and H. W. Jang, Carbon, 2015, 91, 178.

218 A. Salehi-Khojin, D. Estrada, K. Y. Lin, M.-H. Bae, F. Xiong, E. Pop and R. I. Masel, Adv. Mater., 2012, 24, 53.

219 S. Prezioso, F. Perrozzi, L. Giancaterini, C. Cantalini, E. Treossi, V. Palermo, M. Nardone, S. Santucci and L. Ottaviano, J. Phys. Chem. C, 2013, 117, 10683.

220 L. Guo, H.-B. Jiang, R.-Q. Shao, Y.-L. Zhang, S.-Y. Xie, J.-N. Wang, X.-B. Li, F. Jiang, Q.-D. Chen, T. Zhang and H.-B. Sun, Carbon, 2012, 50, 1667.

221 D. Zhang, J. Tong and B. Xia, Sens. Actuators, B, 2014, 197, 66.

222 A. Lipatov, A. Varezhnikov, P. Wilson, V. Sysoev, A. Kolmakov and A. Sinitskii, Nanoscale, 2013, 5, 5426.

223 N. Hu, Y. Wang, J. Chai, R. Gao, Z. Yang, E. S.-W. Kong and Y. Zhang, Sens. Actuators, B, 2012, 163, 107. 
224 V. Dua, S. P. Surwade, S. Ammu, S. R. Agnihotra, S. Jain, K. E. Roberts, S. Park, R. S. Ruoff and S. K. Manohar, Angew. Chem. Int. Ed., 2010, 49, 2154.

225 V. Strong, S. Dubin, M. F. El-Kady, A. Lech, Y. Wang, B. H. Weiller and R. B. Kaner, ACS Nano, 2012, 6, 1395.

226 M. Donarelli, S. Prezioso, F. Perrozzi, L. Giancaterini, C. Cantalini, E. Treossi, V. Palermo, S. Santucci and L. Ottaviano, 2D Mater., 2015, 2, 35018.

227 S. Borini, R. White, D. Wei, M. Astley, S. Haque, E. Spigone, N. Harris, J. Kivioja and T. Ryhänen, ACS Nano, 2013, 7, 11166.

228 K. Rathi and K. Pal, ACS Omega, 2017, $2,842$.

229 Y. Tai, T. K. Bera, G. Lubineau and Z. Yang, J. Mater. Chem. C, 2017, 5, 3848.

230 Y. Yao, X. Chen, J. Zhu, B. Zeng, Z. Wu and X. Li, Nanoscale Res. Lett., 2012, 7, 363.

231 I. Jung, D. Dikin, S. Park, W. Cai, S. L. Mielke and R. S. Ruoff, J. Phys. Chem. C, 2008, 112, 20264.

232 L. Al-Mashat, K. Shin, K. Kalantar-zadeh, J. D. Plessis, S. H. Han, R. W. Kojima, R. B. Kaner, D. Li, X. Gou, S. J. Ippolito and W. Wlodarski, J. Phys. Chem. C, 2010, 114, 16168.

233 X. Huang, N. Hu, R. Gao, Y. Yu, Y. Wang, Z. Yang, E. Siu-Wai Kong, H. Wei and Y. Zhang, J. Mater. Chem., 2012, 22, 22488.

234 W. Yuan, A. Liu, L. Huang, C. Li and G. Shi, Adv. Mater., 2013, 25, 766.

235 A. Kaniyoor, R. Imran Jafri, T. Arockiadoss and S. Ramaprabhu, Nanoscale, 2009, 1, 382.

236 H. Conrad, G. Ertl and E. E. Latta, Surf. Sci., 1974, 41, 435.

237 W. Wu, Z. Liu, L. A. Jauregui, Q. Yu, R. Pillai, H. Cao, J. Bao, Y. P. Chen and S.-S. Pei, Sens. Actuators, B, 2010, 150, 296.

238 M. G. Chung, D.-H. Kim, D. K. Seo, T. Kim, H. U. Im, H. M. Lee, J.-B. Yoo, S.-H. Hong, T. J. Kang and Y. H. Kim, Sens. Actuators, B, 2012, 169, 387.

239 J. Hong, S. Lee, J. Seo, S. Pyo, J. Kim and T. Lee, ACS Appl. Mater. Interfaces, 2015, 7, 3554 .

240 B. H. Chu, C. F. Lo, J. Nicolosi, C. Y. Chang, V. Chen, W. Strupinski, S. J. Pearton and F. Ren, Sens. Actuators, B, 2011, 157, 500.

241 U. Lange, T. Hirsch, V. M. Mirsky and O. S. Wolfbeis, Electrochim. Acta, 2011, 56, 3707.

242 L. Huang, Z. Wang, J. Zhang, J. Pu, Y. Lin, S. Xu, L. Shen, Q. Chen and W. Shi, ACS Appl. Mater. Interfaces, 2014, 6, 7426.

243 S. Deng, V. Tjoa, H. M. Fan, H. R. Tan, D. C. Sayle, M. Olivo, S. Mhaisalkar, J. Wei and C. H. Sow, J. Am. Chem. Soc., 2012, 134, 4905.

244 J. Yi, J. M. Lee and W. I. Park, Sens. Actuators, B, 2011, 155, 264.

245 G. Singh, A. Choudhary, D. Haranath, A. G. Joshi, N. Singh, S. Singh and R. Pasricha, Carbon, 2012, 50, 385.

246 T. V. Cuong, V. H. Pham, J. S. Chung, E. W. Shin, D. H. Yoo, S. H. Hahn, J. S. Huh, G. H. Rue, E. J. Kim, S. H. Hur and P. A. Kohl, Mater. Lett., 2010, 64, 2479.

247 L. Zhou, F. Shen, X. Tian, D. Wang, T. Zhang and W. Chen, Nanoscale, 2013, 5, 1564.

248 S. Mao, S. Cui, G. Lu, K. Yu, Z. Wen and J. Chen, J. Mater. Chem. C, 2012, 22, 11009.

249 J. Liu, S. Li, B. Zhang, Y. Wang, Y. Gao, X. Liang, Y. Wang and G. Lu, J. Colloid Interface Sci., 2017, 504, 206.

250 Q. Huang, D. Zeng, H. Li and C. Xie, Nanoscale, 2012, 4, 5651. 
251 R. K. Mishra, S. B. Upadhyay, A. Kushwaha, T. H. Kim, G. Murali, R. Verma, M. Srivastava, J. Singh, P. P. Sahay and S. H. Lee, Nanoscale, 2015, 7, 11971.

252 G. Eranna, B. C. Joshi, D. P. Runthala and R. P. Gupta, Crit. Rev. Solid State Mater. Sci., 2004, 29, 111.

253 Y.-F. Sun, S.-B. Liu, F.-L. Meng, J.-Y. Liu, Z. Jin, L.-T. Kong and J.-H. Liu, Sensors, 2012, 12, 2610.

254 W. Yang, P. Wan, X. Zhou, J. Hu, Y. Guan and L. Feng, ACS Appl. Mater. Interfaces, 2014, 6, 21093.

255 D. Zhang, H. Chang, P. Li and R. Liu, J. Mater. Sci. Mater. Electron., 2016, 27, 3723.

256 D. Zhang, N. Yin and B. Xia, J. Mater. Sci. Mater. Electron., 2015, 26, 5937.

257 Z. Wu, X. Chen, S. Zhu, Z. Zhou, Y. Yao, W. Quan and B. Liu, IEEE Sens. J., 2013, 13, 777.

258 H. Bai, K. Sheng, P. Zhang, C. Li and G. Shi, J. Mater. Chem., 2011, 21, 18653.

259 W. Yuan, L. Huang, Q. Zhou and G. Shi, ACS Appl. Mater. Interfaces, 2014, 6, 17003.

260 Q. Ji, I. Honma, S.-M. Paek, M. Akada, J. P. Hill, A. Vinu and K. Ariga, Angew. Chem. Int. Ed., 2010, 49, 9737.

261 H. Y. Jeong, D.-S. Lee, H. K. Choi, D. H. Lee, J.-E. Kim, J. Y. Lee, W. J. Lee, S. O. Kim and S.-Y. Choi, Appl. Phys. Lett., 2010, 96, 213105

262 S. Tongay, J. Zhou, C. Ataca, J. Liu, J. S. Kang, T. S. Matthews, L. You, J. Li, J. C. Grossman and J. Wu, Nano Lett., 2013, 13, 2831.

263 B. Cho, J. Yoon, S. K. Lim, A. R. Kim, D. H. Kim, S. G. Park, J. D. Kwon, Y. J. Lee, K. H. Lee, B. H. Lee, H. C. Ko and M. G. Hahm, ACS Appl. Mater. Interfaces, 2015, 7, 16775 .

264 Y. H. Kim, K. Y. Kim, Y. R. Choi, Y.-S. Shim, J.-M. Jeon, J.-H. Lee, S. Y. Kim, S. Han and H. W. Jang, J. Mater. Chem. A, 2016, 4, 6070.

265 Y. Yao, L. Tolentino, Z. Yang, X. Song, W. Zhang, Y. Chen and C.-p. Wong, Adv. Funct. Mater., 2013, 23, 3577.

266 B. Cho, M. G. Hahm, M. Choi, J. Yoon, A. R. Kim, Y.-J. Lee, S.-G. Park, J.-D. Kwon, C. S. Kim, M. Song, Y. Jeong, K.-S. Nam, S. Lee, T. J. Yoo, C. G. Kang, B. H. Lee, H. C. Ko, P. M. Ajayan and D.-H. Kim, Sci. Rep., 2015, 5, 8052.

267 Q. He, Z. Zeng, Z. Yin, H. Li, S. Wu, X. Huang and H. Zhang, Small, 2012, 8, 2994.

268 C. Kuru, C. Choi, A. Kargar, D. Choi, Y. J. Kim, C. H. Liu, S. Yavuz and S. Jin, Adv. Sci., 2015, 2, 1500004

269 D. Sarkar, X. Xie, J. Kang, H. Zhang, W. Liu, J. Navarrete, M. Moskovits and K. Banerjee, Nano Lett., 2015, 15, 2852.

270 S. Cui, Z. Wen, X. Huang, J. Chang and J. Chen, Small, 2015, 11, 2305.

271 H. Yan, P. Song, S. Zhang, Z. Yang and Q. Wang, RSC Adv., 2015, 5, 79593.

272 S. S. Chou, M. De, J. Kim, S. Byun, C. Dykstra, J. Yu, J. Huang and V. P. Dravid, J. Am. Chem. Soc., 2013, 135, 4584.

273 D. J. Late, T. Doneux and M. Bougouma, Appl. Phys. Lett., 2014, 105, 233103

274 Y. Luo, C. Chen, K. Xia, S. Peng, H. Guan, J. Tang, H. Lu, J. Yu, J. Zhang, Y. Xiao and Z. Chen, Opt. Express, 2016, 24, 8956.

275 H. Fang, S. Chuang, T. C. Chang, K. Takei, T. Takahashi and A. Javey, Nano Lett., 2012, 12, 3788.

276 S. Yang, J. Kang, Q. Yue, J. M. D. Coey and C. Jiang, Adv. Mater. Interfaces, 2016, 3, 1500707.

277 S. Yang, S. Tongay, Q. Yue, Y. Li, B. Li and F. Lu, Sci. Rep., 2014, 4, 5442.

278 S. Yang, S. Tongay, Y. Li, Q. Yue, J.-B. Xia, S.-S. Li, J. Li and S.-H. Wei, Nanoscale, 2014, 6, 7226. 
279 C. Yim, K. Lee, N. McEvoy, M. O'Brien, S. Riazimehr, N. C. Berner, C. P. Cullen, J. Kotakoski, J. C. Meyer, M. C. Lemme and G. S. Duesberg, ACS Nano, 2016, 10, 9550.

280 J. Z. Ou, W. Ge, B. J. Carey, T. Daeneke, A. Rotbart, W. Shan, Y. Wang, Z. Fu, A. F. Chrimes, W. Wlodarski, S. P. Russo, Y. X. Li and K. Kalantar-zadeh, ACS Nano, 2015, 9, 10313.

281 S. Weidong, H. Lihua, W. Haishui, Z. Hongjie, Y. Jianhui and W. Pinghui, Nanotechnology, 2006, 17, 2918.

282 P. Yasaei, A. Behranginia, T. Foroozan, M. Asadi, K. Kim, F. Khalili-Araghi and A. Salehi-Khojin, ACS Nano, 2015, 9, 9898.

283 Y.-H. Zhang, K.-G. Zhou, X.-C. Gou, K.-F. Xie, H.-L. Zhang and Y. Peng, Chem. Phys. Lett., 2010, 484, 266.

284 M. Sajjad and P. Feng, Mater. Res. Bull., 2014, 49, 35.

285 S. Chowdhury and R. Balasubramanian, Adv. Colloid Interface Sci., 2014, 204, 35.

286 L. Fan, C. Luo, M. Sun and H. Qiu, J. Mater. Chem., 2012, 22, 24577.

287 H. Wang, X. Yuan, Y. Wu, H. Huang, X. Peng, G. Zeng, H. Zhong, J. Liang and M. Ren, Adv. Colloid Interface Sci., 2013, 195-196, 19.

288 D. S. Sholl and R. P. Lively, Nature, 2016, 532, 435.

289 I. Ali, Chem. Rev., 2012, 112, 5073.

290 T. Zhang, Z. Cheng, Y. Wang, Z. Li, C. Wang, Y. Li and Y. Fang, Nano Lett., 2010, 10, 4738 .

291 H. G. Sudibya, Q. He, H. Zhang and P. Chen, ACS Nano, 2011, 5, 1990.

292 G. Zhou, J. Chang, S. Cui, H. Pu, Z. Wen and J. Chen, ACS Appl. Mater. Interfaces, 2014, 6, 19235.

293 J. H. An, S. J. Park, O. S. Kwon, J. Bae and J. Jang, ACS Nano, 2013, 7, 10563.

294 W. Fu, C. Nef, A. Tarasov, M. Wipf, R. Stoop, O. Knopfmacher, M. Weiss, M. Calame and C. Schonenberger, Nanoscale, 2013, 5, 12104.

295 K. Maehashi, Y. Sofue, S. Okamoto, Y. Ohno, K. Inoue and K. Matsumoto, Sens. Actuators, B, 2013, 187, 45.

296 S. Jiang, R. Cheng, R. Ng, Y. Huang and X. Duan, Nano Res., 2015, 8, 257.

297 G. Zhou, J. Chang, H. Pu, K. Shi, S. Mao, X. Sui, R. Ren, S. Cui and J. Chen, ACS Sens., 2016, 1, 295.

298 P. Li, D. Zhang, C. Jiang, X. Zong and Y. Cao, Biosens. Bioelectron., 2017, 98, 68.

299 Z.-Q. Zhao, X. Chen, Q. Yang, J.-H. Liu and X.-J. Huang, Chem. Commun., 2012, 48, 2180.

300 P. K. Sahoo, B. Panigrahy, S. Sahoo, A. K. Satpati, D. Li and D. Bahadur, Biosens. Bioelectron., 2013, 43, 293.

301 Y. Wei, C. Gao, F.-L. Meng, H.-H. Li, L. Wang, J.-H. Liu and X.-J. Huang, J. Phys. Chem. C, 2012, 116, 1034.

302 J. Li, S. Guo, Y. Zhai and E. Wang, Electrochem. Commun., 2009, 11, 1085.

303 S. Chaiyo, E. Mehmeti, K. Žagar, W. Siangproh, O. Chailapakul and K. Kalcher, Anal. Chim. Acta, 2016, 918, 26.

304 R. Seenivasan, W.-J. Chang and S. Gunasekaran, ACS Appl. Mater. Interfaces, 2015, 7, 15935.

305 J. Cui, S. Xu and L. Wang, Sci. China Mater., 2017, 60, 352.

306 Z. S. Qian, X. Y. Shan, L. J. Chai, J. R. Chen and H. Feng, Biosens. Bioelectron., $2015,68,225$.

307 M. Li, X. Zhou, W. Ding, S. Guo and N. Wu, Biosens. Bioelectron., 2013, 41, 889.

308 Y. Wen, F. Xing, S. He, S. Song, L. Wang, Y. Long, D. Li and C. Fan, Chem. Commun., 2010, 46, 2596. 
309 K. Mao, Z. Wu, Y. Chen, X. Zhou, A. Shen and J. Hu, Talanta, 2015, 132, 658.

310 Y. Yang, T. Liu, L. Cheng, G. Song, Z. Liu and M. Chen, ACS Appl. Mater. Interfaces, 2015, 7, 7526.

311 X. Liu, L. Li, Y. Wei, Y. Zheng, Q. Xiao and B. Feng, Analyst, 2015, 140, 4654.

312 Y. Wang, J. Hu, Q. Zhuang and Y. Ni, ACS Sustain. Chem. Eng., 2016, 4, 2535.

313 X. Zuo, H. Zhang, Q. Zhu, W. Wang, J. Feng and X. Chen, Biosens. Bioelectron., 2016, 85, 464.

314 B. L. Li, J. Wang, H. L. Zou, S. Garaj, C. T. Lim, J. Xie, N. B. Li and D. T. Leong, Adv. Funct. Mater., 2016, 26, 7034.

315 C. Liu, Z. Sun, L. Zhang, J. Lv, X. F. Yu, L. Zhang and X. Chen, Sens. Actuators, B, 2018, 257, 1093.

316 P. Li, D. Zhang, J. Liu, H. Chang, Y. e. Sun and N. Yin, ACS Appl. Mater. Interfaces, 2015, 7, 24396.

317 W. Gu, X. Pei, Y. Cheng, C. Zhang, J. Zhang, Y. Yan, C. Ding and Y. Xian, ACS Sens., 2017, 2, 576.

318 K. C. Kemp, H. Seema, M. Saleh, N. H. Le, K. Mahesh, V. Chandra and K. S. Kim, Nanoscale, 2013, 5, 3149.

319 K. A. Mahmoud, B. Mansoor, A. Mansour and M. Khraisheh, Desalination, 2015, 356, 208.

320 S. Wang, H. Sun, H. M. Ang and M. O. Tadé, Chem. Eng. J., 2013, 226, 336.

321 H. M. Hegab and L. Zou, J. Membr. Sci., 2015, 484, 95.

322 G. Zhao, X. Ren, X. Gao, X. Tan, J. Li, C. Chen, Y. Huang and X. Wang, Dalton Trans., 2011, 40, 10945.

323 W. Peng, H. Li, Y. Liu and S. Song, J. Mol. Liq., 2017, 230, 496.

324 İ. Duru, D. Ege and A. R. Kamali, J. Mater. Sci., 2016, 51, 6097.

325 S. Wang, X. Li, Y. Liu, C. Zhang, X. Tan, G. Zeng, B. Song and L. Jiang, J. Hazard. Mater., 2018, 342, 177.

326 R. Sitko, P. Janik, B. Feist, E. Talik and A. Gagor, ACS Appl. Mater. Interfaces, 2014, 6, 20144.

327 F. Zhang, B. Wang, S. He and R. Man, J. Chem. Eng. Data, 2014, 59, 1719.

328 Y. Yang, Y. Xie, L. Pang, M. Li, X. Song, J. Wen and H. Zhao, Langmuir, 2013, 29, 10727.

329 Y. L. F. Musico, C. M. Santos, M. L. P. Dalida and D. F. Rodrigues, J. Mater. Chem. $A, 2013,1,3789$.

330 L. Hu, Z. Yang, L. Cui, Y. Li, H. H. Ngo, Y. Wang, Q. Wei, H. Ma, L. Yan and B. Du, Chem. Eng. J., 2016, 287, 545.

331 Z. Xu, Y. Zhang, X. Qian, J. Shi, L. Chen, B. Li, J. Niu and L. Liu, Appl. Surf. Sci., 2014, 316, 308.

332 W. Jia and S. Lu, Korean J. Chem. Eng., 2014, 31, 1265.

333 S. Kumar, R. R. Nair, P. B. Pillai, S. N. Gupta, M. A. R. Iyengar and A. K. Sood, ACS Appl. Mater. Interfaces, 2014, 6, 17426.

334 X. Yang, C. Chen, J. Li, G. Zhao, X. Ren and X. Wang, RSC Adv., 2012, 2, 8821.

335 L. Cui, Y. Wang, L. Gao, L. Hu, L. Yan, Q. Wei and B. Du, Chem. Eng. J., 2015, 281, 1.

336 C. J. Madadrang, H. Y. Kim, G. Gao, N. Wang, J. Zhu, H. Feng, M. Gorring, M. L. Kasner and S. Hou, ACS Appl. Mater. Interfaces, 2012, 4, 1186.

337 H. Hadi Najafabadi, M. Irani, L. Roshanfekr Rad, A. Heydari Haratameh and I. Haririan, RSC Adv., 2015, 5, 16532.

338 X. Zou, Y. Yin, Y. Zhao, D. Chen and S. Dong, Mater. Lett., 2015, 150, 59. 
339 X. Li, H. Zhou, W. Wu, S. Wei, Y. Xu and Y. Kuang, J. Colloid Interface Sci., 2015, 448, 389.

340 C. Cheng, S. Li, J. Zhao, X. Li, Z. Liu, L. Ma, X. Zhang, S. Sun and C. Zhao, Chem. Eng. J., 2013, 228, 468.

341 S. Luo, X. Xu, G. Zhou, C. Liu, Y. Tang and Y. Liu, J. Hazard. Mater., 2014, 274, 145.

342 Y. Wang, S. Liang, B. Chen, F. Guo, S. Yu and Y. Tang, PLoS One, 2013, 8, e65634.

343 V. R. Dandu Kamakshi Gari and M. Kim, Monatsh. Chem., 2015, 146, 1445.

344 L. Cui, Y. Wang, L. Hu, L. Gao, B. Du and Q. Wei, RSC Adv., 2015, 5, 9759.

345 G. Zhou, C. Liu, Y. Tang, S. Luo, Z. Zeng, Y. Liu, R. Xu and L. Chu, Chem. Eng. J., 2015, 280, 275.

346 L. Yang, Z. Li, G. Nie, Z. Zhang, X. Lu and C. Wang, Appl. Surf. Sci., 2014, 307, 601.

347 M. Tan, X. Liu, W. Li and H. Li, J. Chem. Eng. Data, 2015, 60, 1469.

348 J. Yang, J.-X. Wu, Q.-F. Lü and T.-T. Lin, ACS Sustain. Chem. Eng., 2014, 2, 1203.

349 Z. Dong, F. Zhang, D. Wang, X. Liu and J. Jin, J. Solid State Chem., 2015, 224, 88.

350 S. Sheshmani, M. Akhundi Nematzadeh, S. Shokrollahzadeh and A. Ashori, Int. J. Biol. Macromol., 2015, 80, 475.

351 R. Sitko, P. Janik, B. Zawisza, E. Talik, E. Margui and I. Queralt, Anal. Chem., 2015, 87, 3535.

352 Y. Q. He, N. N. Zhang and X. D. Wang, Chin. Chem. Lett., 2011, 22, 859.

353 Y. Wang, T. Yan, L. Gao, L. Cui, L. Hu, L. Yan, B. Du and Q. Wei, Desalin. Water Treat., 2016, 57, 3975.

354 L. Fan, C. Luo, M. Sun, X. Li and H. Qiu, Colloids Surf., B, 2013, 103, 523.

355 X. Guo, B. Du, Q. Wei, J. Yang, L. Hu, L. Yan and W. Xu, J. Hazard. Mater., 2014, 278, 211.

356 R. Sitko, B. Zawisza, E. Talik, P. Janik, G. Osoba, B. Feist and E. Malicka, Anal. Chim. Acta, 2014, 834, 22.

357 H. T. Xing, J. H. Chen, X. Sun, Y. H. Huang, Z. B. Su, S. R. Hu, W. Weng, S. X. Li, H. X. Guo, W. B. Wu, Y. S. He, F. M. Li and Y. Huang, Chem. Eng. J., 2015, 263, 280.

358 Y. Yang, W.-Q. Wu, H.-H. Zhou, Z.-Y. Huang, T.-T. Ye, R. Liu and Y.-F. Kuang, J. Cent. South Univ., 2014, 21, 2826.

359 L. Li, Z. Wang, P. Ma, H. Bai, W. Dong and M. Chen, J. Pol. Res., 2015, 22, 150.

360 T. Jiang, W. Liu, Y. Mao, L. Zhang, J. Cheng, M. Gong, H. Zhao, L. Dai, S. Zhang and Q. Zhao, Chem. Eng. J., 2015, 259, 603.

361 W. Wu, Y. Yang, H. Zhou, T. Ye, Z. Huang, R. Liu and Y. Kuang, Water, Air, Soil Pollut., 2012, 224, 1372.

362 I. E. Mejias Carpio, J. D. Mangadlao, H. N. Nguyen, R. C. Advincula and D. F. Rodrigues, Carbon, 2014, 77, 289.

363 D. Chen, H. Zhang, K. Yang and H. Wang, J. Hazard. Mater., 2016, 310, 179.

364 X.-J. Hu, Y.-G. Liu, H. Wang, A.-W. Chen, G.-M. Zeng, S.-M. Liu, Y.-M. Guo, X. Hu, T.-T. Li, Y.-Q. Wang, L. Zhou and S.-H. Liu, Sep. Purif. Technol., 2013, 108, 189.

365 W. M. Algothmi, N. M. Bandaru, Y. Yu, J. G. Shapter and A. V. Ellis, J. Colloid Interface Sci., 2013, 397, 32.

366 Y. Wang, X. Liu, H. Wang, G. Xia, W. Huang and R. Song, J. Colloid Interface Sci., 2014, 416, 243.

367 V. P. Chauke, A. Maity and A. Chetty, J. Mol. Liq., 2015, 211, 71.

368 S. Li, X. Lu, Y. Xue, J. Lei, T. Zheng and C. Wang, PLoS One, 2012, 7, e43328. 
369 Y. Lei, F. Chen, Y. Luo and L. Zhang, J. Mater. Sci., 2014, 49, 4236.

370 A. S. K. Kumar, S. S. Kakan and N. Rajesh, Chem. Eng. J., 2013, 230, 328.

371 H. Ge and Z. Ma, Carbohydr. Polym., 2015, 131, 280.

372 X. Yuan, Y. Wang, J. Wang, C. Zhou, Q. Tang and X. Rao, Chem. Eng. J., 2013, 221, 204.

373 H. Jabeen, V. Chandra, S. Jung, J. W. Lee, K. S. Kim and S. B. Kim, Nanoscale, $2011,3,3583$.

374 L. Li, C. Luo, X. Li, H. Duan and X. Wang, Int. J. Biol. Macromol., 2014, 66, 172.

375 L. Li, H. Duan, X. Wang and C. Luo, New J. Chem., 2014, 38, 6008.

376 L. Li, F. Liu, H. Duan, X. Wang, J. Li, Y. Wang and C. Luo, Colloids Surf., B, 2016, 141, 253.

377 F.-Y. Guo, Y.-G. Liu, H. Wang, G.-M. Zeng, X.-J. Hu, B.-H. Zheng, T.-T. Li, X.-F. Tan, S.-F. Wang and M.-M. Zhang, RSC Adv., 2015, 5, 45384.

378 V. Chandra and K. S. Kim, Chem. Commun., 2011, 47, 3942.

379 Y. Zhang, T. Yan, L. Yan, X. Guo, L. Cui, Q. Wei and B. Du, J. Mol. Liq., 2014, 198, 381.

380 F. Najafi, O. Moradi, M. Rajabi, M. Asif, I. Tyagi, S. Agarwal and V. K. Gupta, J. Mol. Liq., 2015, 208, 106.

381 F. Fang, L. Kong, J. Huang, S. Wu, K. Zhang, X. Wang, B. Sun, Z. Jin, J. Wang, X.-J. Huang and J. Liu, J. Hazard. Mater., 2014, 270, 1.

382 L. Liu, C. Li, C. Bao, Q. Jia, P. Xiao, X. Liu and Q. Zhang, Talanta, 2012, 93, 350.

383 H. Qi, H. Liu and Y. Gao, J. Mol. Liq., 2015, 208, 394.

384 F. Peng, T. Luo, L. Qiu and Y. Yuan, Mater. Res. Bull., 2013, 48, 2180.

385 S. Chen, J. Hong, H. Yang and J. Yang, J. Environ. Radioact., 2013, 126, 253.

386 H. Cheng, K. Zeng and J. Yu, J. Radioanal. Nucl. Chem., 2013, 298, 599.

387 L. Chen, D. Zhao, S. Chen, X. Wang and C. Chen, J. Colloid Interface Sci., 2016, 472, 99.

388 M. Zambianchi, M. Durso, A. Liscio, E. Treossi, C. Bettini, M. L. Capobianco, A. Aluigi, A. Kovtun, G. Ruani, F. Corticelli, M. Brucale, V. Palermo, M. L. Navacchia and M. Melucci, Chem. Eng. J., 2017, 326, 130.

389 H. Gu, S. B. Rapole, Y. Huang, D. Cao, Z. Luo, S. Wei and Z. Guo, J. Mater. Chem. A, 2013, 1, 2011.

390 M. Bhaumik, A. Maity, V. V. Srinivasu and M. S. Onyango, J. Hazard. Mater., 2011, 190, 381 .

391 Y. Wang, B. Zou, T. Gao, X. Wu, S. Lou and S. Zhou, J. Mater. Chem., 2012, 22, 9034.

392 G. Chillemi, G. Mancini, N. Sanna, V. Barone, S. Della Longa, M. Benfatto, N. V. Pavel and P. D'Angelo, J. Am. Chem. Soc., 2007, 129, 5430.

393 M.-R. Gao, M. K. Y. Chan and Y. Sun, Nat. Commun., 2015, 6, 7493.

394 J. Zheng, H. Zhang, S. Dong, Y. Liu, C. Tai Nai, H. Suk Shin, H. Young Jeong, B. Liu and K. Ping Loh, Nat. Commun., 2014, 5, 2995.

395 K. Ai, C. Ruan, M. Shen and L. Lu, Adv. Funct. Mater., 2016, 26, 5542.

396 B. Li, Y. Zhang, D. Ma, Z. Shi and S. Ma, Nat. Commun., 2014, 5, 5537.

397 X. Feng, G. E. Fryxell, L. Q. Wang, A. Y. Kim, J. Liu and K. M. Kemner, Science, 1997, 276, 923.

398 S. Bag, P. N. Trikalitis, P. J. Chupas, G. S. Armatas and M. G. Kanatzidis, Science, 2007, 317, 490.

399 C. Liu, F. Jia, Q. Wang, B. Yang and S. Song, Appl. Mater. Today, 2017, 9, 220.

400 J. Wang, W. Zhang, X. Yue, Q. Yang, F. Liu, Y. Wang, D. Zhang, Z. Li and J. Wang, J. Mater. Chem. A, 2016, 4, 3893. 
401 F. Jia, Q. Wang, J. Wu, Y. Li and S. Song, ACS Sustain. Chem. Eng., 2017, 5, 7410.

402 L. Zhi, W. Zuo, F. Chen and B. Wang, ACS Sustain. Chem. Eng., 2016, 4, 3398.

403 K. P. Carter, A. M. Young and A. E. Palmer, Chem. Rev., 2014, 114, 4564.

404 C. Zhu, D. Du and Y. Lin, 2D Mater., 2015, 2, 32004.

405 E. Treossi, M. Melucci, A. Liscio, M. Gazzano, P. Samorì and V. Palermo, J. Am. Chem. Soc., 2009, 131, 15576.

406 K. Chen, G. Lu, J. Chang, S. Mao, K. Yu, S. Cui and J. Chen, Anal. Chem., 2012, 84, 4057.

407 T. H. Kim, J. Lee and S. Hong, J. Phys. Chem. C, 2009, 113, 19393.

408 Y. Huang, J. Guo, Y. Kang, Y. Ai and C. M. Li, Nanoscale, 2015, 7, 19358.

409 C. M. Willemse, K. Tlhomelang, N. Jahed, P. G. Baker and E. I. Iwuoha, Sensors, 2011, 11, 3970.

410 J. Gong, T. Zhou, D. Song and L. Zhang, Sens. Actuators, B, 2010, 150, 491.

411 G. Lu, H. Li, C. Liusman, Z. Yin, S. Wu and H. Zhang, Chem. Sci., 2011, 2, 1817.

412 M. Zhang, C. Liao, Y. Yao, Z. Liu, F. Gong and F. Yan, Adv. Funct. Mater., 2014, 24, 1036.

413 Z.-H. Sheng, X.-Q. Zheng, J.-Y. Xu, W.-J. Bao, F.-B. Wang and X.-H. Xia, Biosens. Bioelectron., 2012, 34, 125.

414 F. Yan, M. Zhang and J. Li, Adv. Healthc. Mater., 2014, 3, 313.

415 Y. Wang, Y. Shao, D. W. Matson, J. Li and Y. Lin, ACS Nano, 2010, 4, 1790.

416 C. Shan, H. Yang, J. Song, D. Han, A. Ivaska and L. Niu, Anal. Chem., 2009, 81, 2378.

417 X. Kang, J. Wang, H. Wu, I. A. Aksay, J. Liu and Y. Lin, Biosens. Bioelectron., 2009, 25, 901 .

418 C. Shan, H. Yang, D. Han, Q. Zhang, A. Ivaska and L. Niu, Biosens. Bioelectron., 2010, 25, 1070.

419 Z. Wang, X. Zhou, J. Zhang, F. Boey and H. Zhang, J. Phys. Chem. C, 2009, 113, 14071.

420 Y. Liu, D. Yu, C. Zeng, Z. Miao and L. Dai, Langmuir, 2010, 26, 6158.

421 J. Luo, S. Jiang, H. Zhang, J. Jiang and X. Liu, Anal. Chim. Acta, 2012, 709, 47.

422 H. Wu, J. Wang, X. Kang, C. Wang, D. Wang, J. Liu, I. A. Aksay and Y. Lin, Talanta, 2009, 80, 403.

423 T. T. Baby, S. S. J. Aravind, T. Arockiadoss, R. B. Rakhi and S. Ramaprabhu, Sens. Actuators, B, 2010, 145, 71.

424 S. Alwarappan, A. Erdem, C. Liu and C.-Z. Li, J. Phys. Chem. C, 2009, 113, 8853.

425 G. Zeng, Y. Xing, J. Gao, Z. Wang and X. Zhang, Langmuir, 2010, 26, 15022.

426 K. Zhou, Y. Zhu, X. Yang and C. Li, Electroanalysis, 2010, 22, 259.

427 N. Q. Dung, D. Patil, T. T. Duong, H. Jung, D. Kim and S.-G. Yoon, Sens. Actuators, $B, 2012, \mathbf{1 6 6}, 103$.

428 X. Dong, Y. Shi, W. Huang, P. Chen and L.-J. Li, Adv. Mater., 2010, 22, 1649.

429 M. Du, T. Yang and K. Jiao, J. Mater. Chem., 2010, 20, 9253.

430 O. Niwa, J. Jia, Y. Sato, D. Kato, R. Kurita, K. Maruyama, K. Suzuki and S. Hirono, J. Am. Chem. Soc., 2006, 128, 7144.

431 C. Wang, L. Zhang, Z. Guo, J. Xu, H. Wang, K. Zhai and X. Zhuo, Microchim. Acta, 2010, 169, 1 .

432 N. G. Shang, P. Papakonstantinou, M. McMullan, M. Chu, A. Stamboulis, A. Potenza, S. S. Dhesi and H. Marchetto, Adv. Funct. Mater., 2008, 18, 3506.

433 Y.-R. Kim, S. Bong, Y.-J. Kang, Y. Yang, R. K. Mahajan, J. S. Kim and H. Kim, Biosens. Bioelectron., 2010, 25, 2366. 
434 C.-L. Sun, H.-H. Lee, J.-M. Yang and C.-C. Wu, Biosens. Bioelectron., 2011, 26, 3450.

435 C. X. Lim, H. Y. Hoh, P. K. Ang and K. P. Loh, Anal. Chem., 2010, 82, 7387.

436 S. Hou, M. L. Kasner, S. Su, K. Patel and R. Cuellari, J. Phys. Chem. C, 2010, 114, 14915.

437 L. Wu, L. Feng, J. Ren and X. Qu, Biosens. Bioelectron., 2012, 34, 57.

438 L. Tan, K.-G. Zhou, Y.-H. Zhang, H.-X. Wang, X.-D. Wang, Y.-F. Guo and H.-L. Zhang, Electrochem. Commun., 2010, 12, 557.

439 F. Li, J. Chai, H. Yang, D. Han and L. Niu, Talanta, 2010, 81, 1063.

440 Y. Fan, H.-T. Lu, J.-H. Liu, C.-P. Yang, Q.-S. Jing, Y.-X. Zhang, X.-K. Yang and K.J. Huang, Colloids Surf., B, 2011, 83, 78.

441 T. Peik-See, A. Pandikumar, H. Nay-Ming, L. Hong-Ngee and Y. Sulaiman, Sensors 2014, 14, 15227.

442 G. P. Keeley, A. O'Neill, M. Holzinger, S. Cosnier, J. N. Coleman and G. S. Duesberg, Phys. Chem. Chem. Phys., 2011, 13, 7747.

443 Y. Fang, S. Guo, C. Zhu, Y. Zhai and E. Wang, Langmuir, 2010, 26, 11277.

444 S. Karapetis, S. Bratakou, G.-P. Nikoleli, C. Siontorou, D. Nikolelis and N. Tzamtzis, Electroanalysis, 2016, 28, 2171.

445 Y. Huang, X. Dong, Y. Shi, C. M. Li, L.-J. Li and P. Chen, Nanoscale, 2010, 2, 1485.

446 Y. H. Kwak, D. S. Choi, Y. N. Kim, H. Kim, D. H. Yoon, S.-S. Ahn, J.-W. Yang, W. S. Yang and S. Seo, Biosens. Bioelectron., 2012, 37, 82.

447 W. Fu, L. Feng, G. Panaitov, D. Kireev, D. Mayer, A. Offenhäusser and H.-J. Krause, Sci. Adv., 2017, 3, 1701247.

448 G. Liu, S. Rumyantsev, M. S. Shur and A. A. Balandin, Appl. Phys. Lett., 2013, 102, 93111.

449 B. Cai, S. Wang, L. Huang, Y. Ning, Z. Zhang and G.-J. Zhang, ACS Nano, 2014, 8, 2632.

450 P. Alonso-Cristobal, P. Vilela, A. El-Sagheer, E. Lopez-Cabarcos, T. Brown, O. L. Muskens, J. Rubio-Retama and A. G. Kanaras, ACS Appl. Mater. Interfaces, 2015, 7, 12422.

451 H. Dong, W. Gao, F. Yan, H. Ji and H. Ju, Anal. Chem., 2010, 82, 5511.

452 C. Liu, Z. Wang, H. Jia and Z. Li, Chem. Commun., 2011, 47, 4661.

453 L. Fan, Y. Hu, X. Wang, L. Zhang, F. Li, D. Han, Z. Li, Q. Zhang, Z. Wang and L. Niu, Talanta, 2012, 101, 192.

454 J.-L. Chen, X.-P. Yan, K. Meng and S.-F. Wang, Anal. Chem., 2011, 83, 8787.

455 C.-H. Lu, H.-H. Yang, C.-L. Zhu, X. Chen and G.-N. Chen, Angew. Chem. Int. Ed., 2009, 48, 4785.

456 Y. Lin, Y. Tao, F. Pu, J. Ren and X. Qu, Adv. Funct. Mater., 2011, 21, 4565.

457 F. Li, Y. Huang, Q. Yang, Z. Zhong, D. Li, L. Wang, S. Song and C. Fan, Nanoscale, 2010, 2, 1021.

458 X. Liu, L. Cao, W. Song, K. Ai and L. Lu, ACS Appl. Mater. Interfaces, 2011, 3, 2944.

459 Z. Chen, L. Qiu, Y. Tian, Y.-I. Lee, X. Hou and L. Wu, Anal. Methods, 2017, 9, 3105.

460 P. V. Shanta and Q. Cheng, ACS Sens., 2017, $2,817$.

461 Y. Song, K. Qu, C. Zhao, J. Ren and X. Qu, Adv. Mater., 2010, 22, 2206.

462 S. Alwarappan, C. Liu, A. Kumar and C.-Z. Li, J. Phys. Chem. C, 2010, 114, 12920.

463 P. Wu, Q. Shao, Y. Hu, J. Jin, Y. Yin, H. Zhang and C. Cai, Electrochim. Acta, 2010, 55, 8606.

464 Y. Zhang, Y. Wang, J. Jia and J. Wang, Sens. Actuators, B, 2012, 171, 580. 
465 M. Zhang, C. Liao, C. H. Mak, P. You, C. L. Mak and F. Yan, Sci. Rep., 2015, 5, 8311.

466 S. He, B. Song, D. Li, C. Zhu, W. Qi, Y. Wen, L. Wang, S. Song, H. Fang and C. Fan, Adv. Funct. Mater., 2010, 20, 453.

467 J. Balapanuru, J.-X. Yang, S. Xiao, Q. Bao, M. Jahan, L. Polavarapu, J. Wei, Q.-H. $\mathrm{Xu}$ and K. P. Loh, Angew. Chem. Int. Ed., 2010, 49, 6549.

468 Z. Wang, J. Zhang, P. Chen, X. Zhou, Y. Yang, S. Wu, L. Niu, Y. Han, L. Wang, P. Chen, F. Boey, Q. Zhang, B. Liedberg and H. Zhang, Biosens. Bioelectron., 2011, 26, 3881.

469 C.-H. Lu, J. Li, J.-J. Liu, H.-H. Yang, X. Chen and G.-N. Chen, Chem. - Eur. J., 2010, 16, 4889.

470 R. Devasenathipathy, V. Mani and S.-M. Chen, Talanta, 2014, 124, 43.

471 J. Ding, S. Zhu, T. Zhu, W. Sun, Q. Li, G. Wei and Z. Su, RSC Adv., 2015, 5, 22935.

472 F. Gao, Q. Wang, N. Gao, Y. Yang, F. Cai, M. Yamane, F. Gao and H. Tanaka, Biosens. Bioelectron., 2017, 97, 238.

473 Z. Yang, Q. Sheng, S. Zhang, X. Zheng and J. Zheng, Microchim. Acta, 2017, 184, 2219.

474 Y. Wang, Y. Li, L. Tang, J. Lu and J. Li, Electrochem. Commun., 2009, 11, 889.

475 J. Du, R. Yue, F. Ren, Z. Yao, F. Jiang, P. Yang and Y. Du, Gold Bull., 2013, 46, 137.

476 J. Ping, J. Wu, Y. Wang and Y. Ying, Biosens. Bioelectron., 2012, 34, 70.

477 G. P. Keeley, A. O'Neill, N. McEvoy, N. Peltekis, J. N. Coleman and G. S. Duesberg, J. Mater. Chem., 2010, 20, 7864.

478 S. Guo, D. Wen, Y. Zhai, S. Guo and E. Wang, ACS Nano, 2010, 4, 3959.

479 K. Zhou, Y. Zhu, X. Yang, J. Luo, C. Li and S. Luan, Electrochim. Acta, 2010, 55, 3055.

480 L. Li, Z. Du, S. Liu, Q. Hao, Y. Wang, Q. Li and T. Wang, Talanta, 2010, 82, 1637.

481 A. Ciesielski and P. Samorì, Chem. Soc. Rev., 2014, 43, 381.

482 J. N. Coleman, Acc. Chem. Res., 2013, 46, 14.

483 F. Bonaccorso, A. Lombardo, T. Hasan, Z. Sun, L. Colombo and A. C. Ferrari, Mater. Today, 2012, 15, 564.

484 J. Wang, Chem. Rev., 2008, 108, 814.

485 L. Tang, Y. Wang, Y. Li, H. Feng, J. Lu and J. Li, Adv. Funct. Mater., 2009, 19, 2782.

486 S. Wu, Z. Zeng, Q. He, Z. Wang, S. J. Wang, Y. Du, Z. Yin, X. Sun, W. Chen and H. Zhang, Small, 2012, 8, 2264.

487 N. N. Thrangattu, S. R. V. Chiranjeevi and A. Subbiah, Nanotechnology, 2014, 25, 409601.

488 T. Wang, H. Zhu, J. Zhuo, Z. Zhu, P. Papakonstantinou, G. Lubarsky, J. Lin and M. Li, Anal. Chem., 2013, 85, 10289.

489 X. Wang, F. Nan, J. Zhao, T. Yang, T. Ge and K. Jiao, Biosens. Bioelectron., 2015, 64, 386.

490 G.-X. Wang, W.-J. Bao, J. Wang, Q.-Q. Lu and X.-H. Xia, Electrochem. Commun., 2013, 35, 146.

491 J. Tang, Y. Quan, Y. Zhang, M. Jiang, A. M. Al-Enizi, B. Kong, T. An, W. Wang, L. Xia, X. Gong and G. Zheng, Nanoscale, 2016, 8, 5786.

492 M. Kukkar, A. Sharma, P. Kumar, K.-H. Kim and A. Deep, Anal. Chim. Acta, 2016, 939, 101.

493 S. Su, H. Sun, F. Xu, L. Yuwen, C. Fan and L. Wang, Microchim. Acta, 2014, 181, 1497.

494 S. Su, H. Sun, F. Xu, L. Yuwen and L. Wang, Electroanalysis, 2013, 25, 2523. 
495 J. Chao, X. Han, H. Sun, S. Su, L. Weng and L. Wang, Sci. China Chem., 2016, 59, 332.

496 K.-J. Huang, Y.-J. Liu, J.-Z. Zhang and Y.-M. Liu, Anal. Methods, 2014, 6, 8011.

497 J. Huang, Y. He, J. Jin, Y. Li, Z. Dong and R. Li, Electrochim. Acta, 2014, 136, 41.

498 R. Yang, J. Zhao, M. Chen, T. Yang, S. Luo and K. Jiao, Talanta, 2015, 131, 619.

499 T. Lin, L. Zhong, L. Guo, F. Fu and G. Chen, Nanoscale, 2014, 6, 11856.

500 T. Lin, L. Zhong, Z. Song, L. Guo, H. Wu, Q. Guo, Y. Chen, F. Fu and G. Chen, Biosens. Bioelectron., 2014, 62, 302.

501 Y. Zhang, B. Zheng, C. Zhu, X. Zhang, C. Tan, H. Li, B. Chen, J. Yang, J. Chen, Y. Huang, L. Wang and H. Zhang, Adv. Mater., 2015, 27, 935.

502 C. Tan, P. Yu, Y. Hu, J. Chen, Y. Huang, Y. Cai, Z. Luo, B. Li, Q. Lu, L. Wang, Z. Liu and H. Zhang, J. Am. Chem. Soc., 2015, 137, 10430.

503 J. Huang, L. Ye, X. Gao, H. Li, J. Xu and Z. Li, J. Mater. Chem. B, 2015, 3, 2395.

504 J. Lee, P. Dak, Y. Lee, H. Park, W. Choi, M. A. Alam and S. Kim, Sci. Rep., 2014, 4, 7352.

505 H.-Y. Park, S. R. Dugasani, D.-H. Kang, G. Yoo, J. Kim, B. Gnapareddy, J. Jeon, M. Kim, Y. J. Song, S. Lee, J. Heo, Y. J. Jeon, S. H. Park and J.-H. Park, Sci. Rep., 2016, 6, 35733 .

506 D.-W. Lee, J. Lee, I. Y. Sohn, B.-Y. Kim, Y. M. Son, H. Bark, J. Jung, M. Choi, T. H. Kim, C. Lee and N.-E. Lee, Nano Res., 2015, 8, 2340.

507 Kenry, A. Geldert, Z. Lai, Y. Huang, P. Yu, C. Tan, Z. Liu, H. Zhang and C. T. Lim, Small, 2017, 13, 1601925.

508 H. Sun, J. Chao, X. Zuo, S. Su, X. Liu, L. Yuwen, C. Fan and L. Wang, RSC Adv., 2014, 4, 27625.

509 X. Xia, Z. Zheng, Y. Zhang, X. Zhao and C. Wang, Sens. Actuators, B, 2014, 192, 42.

510 H. Song, Y. Ni and S. Kokot, Biosens. Bioelectron., 2014, 56, 137.

511 K.-J. Huang, L. Wang, J. Li and Y.-M. Liu, Sens. Actuators, B, 2013, 178, 671.

512 Y. Huang, Y. Shi, H. Y. Yang and Y. Ai, Nanoscale, 2015, 7, 2245.

513 J. Shan, J. Li, X. Chu, M. Xu, F. Jin, X. Wang, L. Ma, X. Fang, Z. Wei and X. Wang, $R S C A d v ., 2018,8,7942$.

514 X. Hu, W. Shao, X. Hang, X. Zhang, W. Zhu and Y. Xie, Angew. Chem. Int. Ed., 2016, 55, 5733.

515 J. Lu, Carbon, 2007, 45, 1599.

516 K. Chang and W. Chen, Chem. Commun., 2011, 47, 4252.

517 K. Chang and W. Chen, ACS Nano, 2011, 5, 4720.

518 E. G. Da Silveira Firmiano, A. C. Rabelo, C. J. Dalmaschio, A. N. Pinheiro, E. C. Pereira, W. H. Schreiner and E. R. Leite, Adv. Energy Mater., 2014, 4, 1301380.

519 X. Zhao, X. Xia, S. Yu and C. Wang, Anal. Methods, 2014, 6, 9375.

520 K. Pramoda, K. Moses, U. Maitra and C. N. R. Rao, Electroanalysis, 2015, 27, 1892.

521 K. Duan, Y. Du, Q. Feng, X. Ye, H. Xie, M. Xue and C. Wang, ChemCatChem, 2014, 6, 1873.

522 H.-L. Shuai, K.-J. Huang and Y.-X. Chen, J. Mater. Chem. B, 2016, 4, 1186.

523 K. Zhang, H. Sun and S. Hou, Anal. Methods, 2016, 8, 3780.

524 A. F. Khan, D. A. C. Brownson, E. P. Randviir, G. C. Smith and C. E. Banks, Anal. Chem., 2016, 88, 9729.

525 A. F. Khan, D. A. C. Brownson, C. W. Foster, G. C. Smith and C. E. Banks, Analyst, 2017, 142, 1756.

526 Y. Zhan, J. Yan, M. Wu, L. Guo, Z. Lin, B. Qiu, G. Chen and K.-y. Wong, Talanta, 2017, 174, 365. 
527 Q. Cai, L. H. Li, Y. Yu, Y. Liu, S. Huang, Y. Chen, K. Watanabe and T. Taniguchi, Phys. Chem. Chem. Phys., 2015, 17, 7761.

528 R. B. Rakhi, P. Nayak, C. Xia and H. N. Alshareef, Sci. Rep., 2016, 6, 36422.

529 F. Wang, C. Yang, M. Duan, Y. Tang and J. Zhu, Biosens. Bioelectron., 2015, 74, 1022.

530 S. Yan, B. Wang, Z. Wang, D. Hu, X. Xu, J. Wang and Y. Shi, Biosens. Bioelectron., 2016, 80, 34.

531 Y. T. Yew, Z. Sofer, C. C. Mayorga-Martinez and M. Pumera, Mater. Chem. Front., 2017, 1, 1130. 